Portland State University

PDXScholar

$5-29-2020$

\title{
Identifying a Starting Point for the Guided Reinvention of the Classification of Chemically Important Symmetry Groups
}

Anna Marie Bergman

Portland State University

Follow this and additional works at: https://pdxscholar.library.pdx.edu/open_access_etds

Part of the Science and Mathematics Education Commons Let us know how access to this document benefits you.

Recommended Citation

Bergman, Anna Marie, "Identifying a Starting Point for the Guided Reinvention of the Classification of Chemically Important Symmetry Groups" (2020). Dissertations and Theses. Paper 5477.

https://doi.org/10.15760/etd.7349

This Dissertation is brought to you for free and open access. It has been accepted for inclusion in Dissertations and Theses by an authorized administrator of PDXScholar. Please contact us if we can make this document more accessible: pdxscholar@pdx.edu. 
Identifying a Starting Point for the Guided Reinvention of the Classification of Chemically Important Symmetry Groups

by

Anna Marie Bergman

A dissertation submitted in partial fulfillment of the requirements for the degree of

\author{
Doctor of Philosophy \\ in \\ Mathematics Education
}
Dissertation Committee:
Sean Larsen, Chair
John Caughman
Paul Latiolais
Andrea Goforth
Joel Steele

Portland State University

2020 


\begin{abstract}
The study of abstract algebra is both required for most mathematics majors and notoriously difficult. Much of the mathematics education literature on investigating student thinking in abstract algebra highlights student struggles with understanding even the most fundamental concepts. The abstract nature of the content of the course has been credited as one of the contributors to student difficulties. While there have been various instructional innovations designed to support students in better understanding abstract algebra, and group theory in particular, they have not specifically focused on the issue of the abstract nature of the content. My dissertation study aimed to develop an instructional theory based on a real-world application of group theory in order to support students in deepening their understanding of abstract algebra. For this study I conducted three teaching experiments with pairs of mathematics students, producing over 35 hours of video data and 235 pages of student inscriptions. The first experiment invited graduate students with ample abstract algebra experience, the second had undergraduates who had recently completed an introductory group theory course, and the third experiment invited undergraduates with no previous exposure to abstract algebra. The study was conducted using the instructional design theory of realistic mathematics education which supplied both an underlying theoretical perspective and accompanying design heuristics. The results from this study are broken into three papers. The first paper reports on the findings of the first teaching experiment and was written for a chemistry education audience. The second paper introduces the local instructional theory (LIT) that was developed over the entirety of the study. The LIT is a generalized sequence of steps for the guided reinvention of a classification system for chemically important symmetry
\end{abstract}


groups. The final paper highlights differences in the students' mathematical activity while engaging in the LIT due to the differences in their mathematical backgrounds and how the LIT was used to support their success. 


\section{Acknowledgements}

First and foremost, thank you to my mentor, doctoral advisor, and friend Sean Larsen. Sean you have taught me so much over this doctoral process and as I sit and reflect, three things in particular come to mind. First of all, thank you for making this dissertation amazing. You have helped me to create something that I am really proud of and I have learned so much from this experience that I will use throughout my career. Second, thank you for helping me learn to be an academic (and human). At times this can be a scary and overwhelming process, thank you for helping me to navigate it all. I cherish your advice and I look forward to mentoring one day to pay it forward. Third, and most importantly thank you for teaching me I could do math. I always wanted to do math and

I even tried by getting a few degrees in it, but it wasn't until your class that someone both supported me and held me accountable. For too long, I was the student who attacked math as list of procedures to be memorized and strategically applied, by the time I got to my doctorate I was exhausted and running out of RAM, then you showed me that math was more, so, so much more. You are orange through and through Sean Larsen, a real life legendary.

Thank you to my dissertation committee for your support over the years and especially in my dissertation. Paul, thank you for your friendship and endless support for so, so many years. John, thank you for your attention to detail genuine interest in my work. Andrea, thank you for allowing me to sit in on your class and ask so many strange questions. Thank you for your excitement in my project and I look forward to working together again soon. Joel, thank you for being a part of my team; it was a real treat to have your insight on my dissertation study. Overall, I appreciate the time and energy you 
have each spent both being a part of my dissertation process, and also in making me feel like my work is special and exciting.

To my participants THANK YOU! Thank you for thinking out loud, thank you for sharing your ideas, thank you for loving to learn, and thank you for making the whole experience so much fun. It really was a joy to do math with each and every one of you.

Thank you to Dr. Kate Melhuish for introducing me to the study of student thinking in group theory, I am forever grateful. You once told me that there was a career where, "I either get to think about math, or think about people thinking about math" and from that moment on I finally knew what I wanted to be when I grew up. Thank you. Thank you to my MWPR group, knowing that a group of such amazingly brilliant women see me as one of their own has given me confidence that knows no bound, and for this dissertation I really needed that. Thank you, friends.

This work has introduced me to a wonderful community of DBER researchers, thank you all for your welcomeness and your ongoing excitement for my efforts. More specifically, thank you to the executive committee of the TRUSE conference for awarding my TRUSE Collaborative Travel Grant. Thank you to Dr. Tim French for being an amazing coauthor. Working and writing with you was such a wonderful experience and I couldn't have asked for a better introduction to interdisciplinary collaboration, thank you.

Thank you to all of my friends and family who've graciously accepted my absence over the years while "I have to work." I can't wait to start reconnecting with you all. In particular thank you to David Brown, Naneh Apkarian, and Dana Kirin. David thank you for your hours and hours of work helping me collect data, and then also listening to me 
think about it. I hope the intimate experience of helping on a dissertation was a fraction as useful to you as it was to me. Naneh, thank you for being such an amazing friend and calm, steady, role model in academia, I'm just so jazzed to do this career thing with you. Glover, we've been doing this a long time, and now we're doctors?!?! Say what??? Thanks for being there the whole way. Thank you to Dana, for being my doctoral bestie. I am as thankful for the friendship we've built over my time in the program as I am for the math I've learned. You are one of the dearest and most important resources I have accumulated in this process and I am so thankful for you.

Thank you to my overly ambitious family for making awesomeness a norm, and for continually supporting me through my long journey in academia with an extra special thank you to my parents. Mom, Dad, Charles, you have always taught me that if I worked really hard, I could do anything. You were sharing the growth mindset message way before it was mainstream, and it has shaped my life into one of endless possibilities. Thank you. Thank you for always being proud of me and never missing an opportunity to share it, every time you did, it helped.

Thank you to my husband Ryan for your nonstop support throughout my entire doctoral process. You've only known me as a graduate student, and I cannot wait to see what life has in store for us after this. Thank you for your patience while I've chased my dream. Lastly, to the dogs. To my Darwin, Stryder, and Bob this dissertation would never have been finished had it not been for your consist reminders that I need balance to move forward. For every mind-clearing, thought provoking, soul satisfying, smile inducing, mud clad walk, run, and bike ride around the greater Pacific North West, thank you, thank you, thank you. 


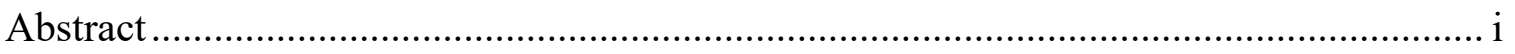

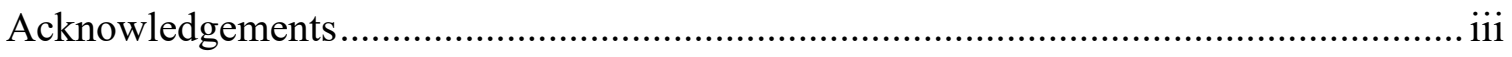

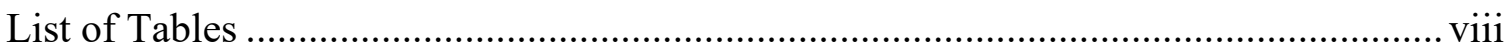

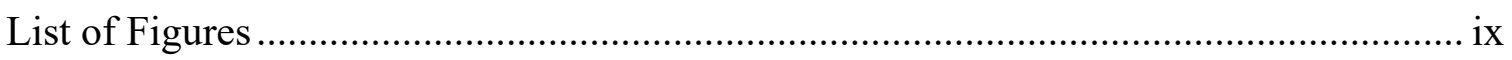

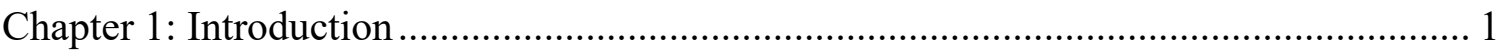

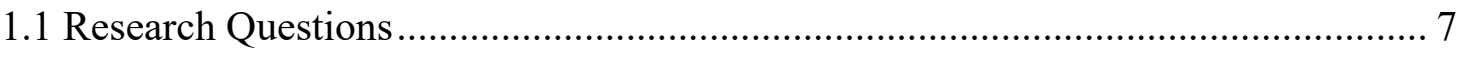

Chapter 2: Literature Review .................................................................................. 11

2.1 - Mathematics Education Literature on Abstract Algebra ...................................... 11

2.1.1 - Student Thinking in Abstract Algebra ..................................................... 12

2.1.2 - An Argument for Teaching Abstract Algebra with Symmetry Groups........ 26

2.2 - Group Theory in Undergraduate Chemistry ..................................................... 31

2.2.1 - Chemistry Student's Thinking Related to Symmetries.................................. 33

2.2.2 - Instructional Innovations for Chemically Important Group Theory and Point

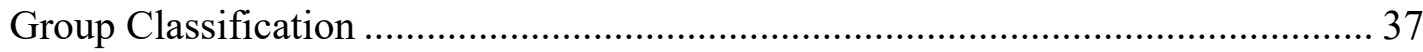

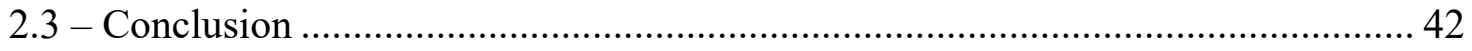

Chapter 3: Theoretical Perspectives........................................................................... 44

3.1 - Realistic Mathematics Education.................................................................... 44

3.1.1 - Theoretical Perspective: Mathematics as a Human Activity ......................... 44

3.1.2 - Theoretical Framework: The Three Design Heuristics of RME.................... 46

3.1.3 - Local Instructional Theories ………………………................................. 52

3.1.4 - The Ongoing Development of Realistic Mathematics Education.................. 54

Chapter 4: Study Methods and Design ..................................................................... 57

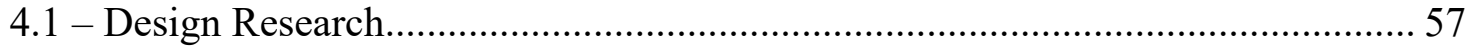

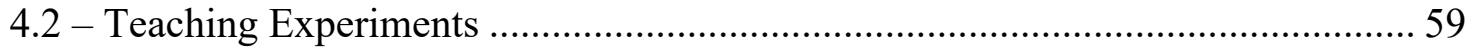

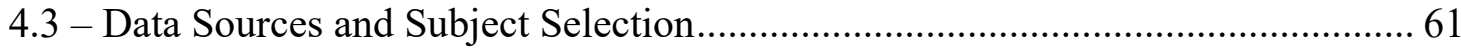

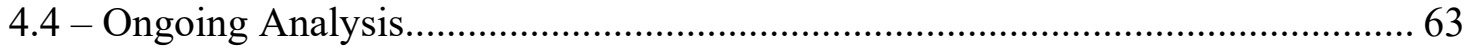

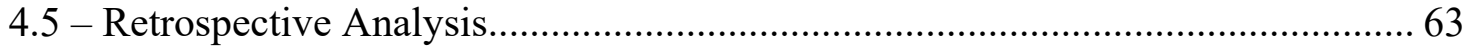

Chapter 5: Paper 1: Developing an Active Approach to Chemistry Based Group Theory

Chapter 6: Paper 2: A Local Instructional Theory for the Guided Reinvention of a Classification Algorithm for Chemically Important Symmetry Groups. 
Chapter 7: Paper 3: Productively Applying and Adapting a LIT for Different Kinds of

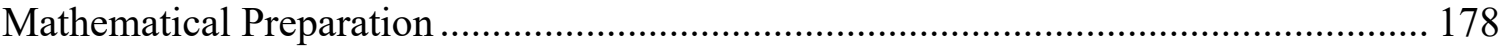

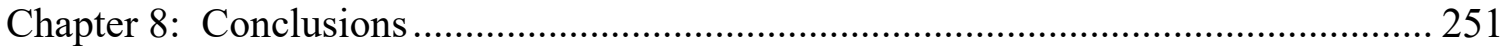

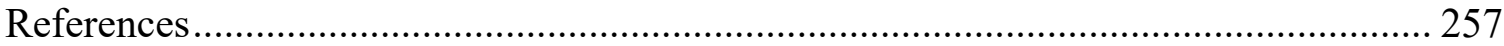

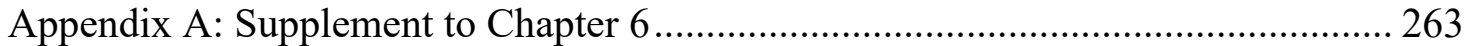

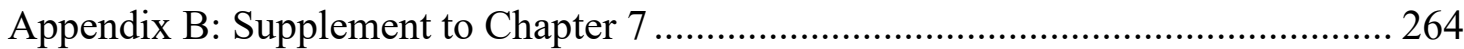

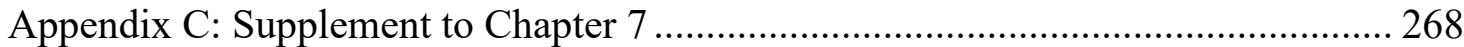




\section{List of Tables}

Table 1. A summary of the three teaching experiments 63

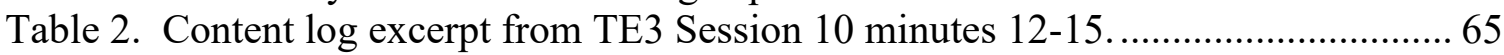
Table 3. Evolution of the students' descriptions of symmetries. Students' own words are italicized. 98

Table 4. Summary table for symmetry elements and operations typically used in an inorganic chemistry course

Table 5. A full collection of chemically relevant symmetry groups represented with both mathematical group names and chemical group names

Table 6. An overview of each of the three teaching experiments.

Table 7. A local instructional theory for the guided reinvention of a classification algorithm for chemically important symmetry groups

Table 8. A local instructional theory for the guided reinvention of a classification algorithm for chemically important symmetry groups

Table 9. Example of a content log used for data analysis and organization. 


\section{List of Figures}

Figure 1. The symmetries of a water molecule; which include an order 2 rotational axis and two vertical planes.

Figure 2. A typical point group classification flowchart given to inorganic chemistry

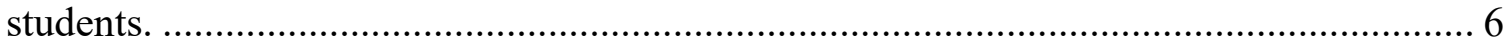

Figure 3. Visualization/Analysis Model (Zazkis et al., 1996, p. 447) ........................... 20 Figure 4. The trainees' introduction to the theorem of two reflections (Almeida, 1999, p. 162). 22

Figure 5. The undergraduates' introduction to the theorem of two reflections (Almeida,

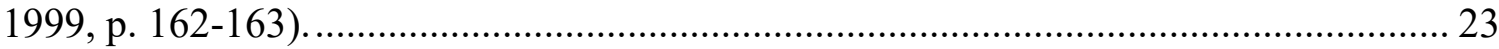

Figure 6. Selected models and manipulatives found in chemistry education literature.... 41 Figure 7. Levels of mathematical activity (Gravemeijer, 1998, p.286-287)................... 50 Figure 8. The design research cycle (Wawro, Rasmussen, Zandieh, \& Larson., 2013b) 57

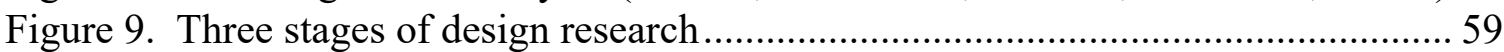
Figure 10. A typical flowchart given to undergraduates in inorganic chemistry to aid in determining the symmetry groups of molecular shapes. Adapted from (45). ............... 85 Figure 11. Initial task prompt and accompanying manipulatives................................... 86 Figure 12. Emmy considers a 180 degree rotation of a water molecule while describing

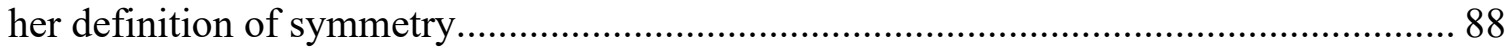

Figure 13. Emmy investigating the effect of "slices", often referred to as horizontal reflections, on ethane.

Figure 14. Emmy considers the effects of vertical and horizontal reflections on particular atoms.

Figure 15. Examples of the students' 'dot diagram' used to determine equivalent symmetries found in the eclipsed configuration of ethane. Figure 16. Initial model for finding symmetry groups. (Left) Inscriptions associated with the pilot students' initial model-of their approach. (Right) The researcher's (AMB) model-of the students model using more conventional terminology. Figure 17. The evolution of the students' initial model through the refining process. The student's observation upon reflection and its corresponding effect on the model are shown for three iterations. Note that the phrase "cyclic group" here refers to a mathematical context, rather than a chemical one.

Figure 18. Intermediate model for finding symmetry groups. (Left) Inscriptions associated with the pilot students' intermediate model-of their approach. (Right) The researcher's (AMB) model-of the students model using more conventional terminology.

Figure 19. Final model for finding symmetry groups. (Left) Inscriptions associated with the pilot students' intermediate model-of their approach. (Right) The researcher's (AMB) model-of the students model using more conventional terminology.

Figure 20. The traditional flowchart, as seen in Figure 1, where the shaded portion corresponds to the symmetry group structures that are identifiable by the algorithm 'reinvented' by the students in the pilot study.

Figure 21. A typical point group classification flowchart given to inorganic chemistry students. 
Figure 22. A typical flowchart given to inorganic chemistry students with mathematical group names instead of chemical group names ........................................................ 117

Figure 23. The initial prompt given to participants in TE2 ………………............... 126

Figure 24. Arthur's gesture indicating a reflection plane in water............................... 127

Figure 25. Stu indicating rotational axes in water. ................................................. 128

Figure 26. Stu indicating a rotational axis in ammonia............................................. 129

Figure 27. Stu demonstrating a reflection with a hand gesture..................................... 130

Figure 28. Arthur using ball and stick models of Tetra-aza Copper II (left) and ethane

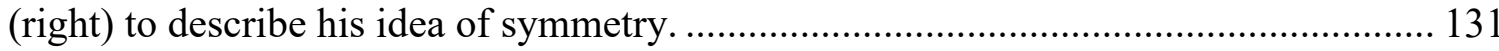

Figure 29. The initial prompt given to students in TE3 ............................................ 133

Figure 30. Students' geometric representations of symmetry group elements for water, ammonia, and ethane from TE3 ............................................................................. 135

Figure 31. Arthur and Stu's initial Cayley table for (a subset of) the symmetries of ethane

used to discover new symmetry elements.................................................................... 137

Figure 32. Emmy and Felix's Cayley table for ethane (in an eclipsed configuration)... 139

Figure 33. Preliminary chain of signification for Model 1.......................................... 141

Figure 34. Students' group presentations for the symmetry group of water, ammonia, and

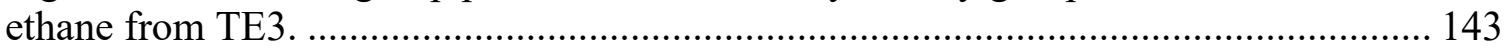

Figure 35. Additional molecules given to students in TE3 along with the group

presentations created to describe each.

Figure 36. Student's "Score Cards" containing a group presentation for each molecule they have investigated, TE3.

Figure 37. Students sorting of their score cards into four group types along with the

names assigned to each type of symmetry group, TE3.

Figure 38. Prompt given to students in TE3 to promote the explication of their system for determining a symmetry group.

Figure 39. Student's work associated with trying to find commonalities between group structures, TE3.

Figure 40. Ada and Sophie's initial attempts at explicating a system for determining a symmetry group, TE3.

Figure 41. Second Iterations of Ada and Sophie's model for a system for determining symmetry groups in general, TE3...

Figure 42. Arthur and Stu's initial model for a system for determining symmetry groups in general, TE2.

Figure 43. Additional molecules given to students to test their system for identifying symmetry groups in TE3.

Figure 44. Prompt given to Ada and Sophie during Step 4 of TE3 .............................. 156

Figure 45. Ada and Sophie's model for identifying the symmetry group of a given molecule incorporating their newly created group names, TE3.

Figure 46. A description of ethane in either the eclipsed or staggered configurations... 158 Figure 47. Prompt given to students in TE1, TE2, and TE3 .......................................... 159

Figure 48. Student's written work while investigating an inversion in ethane. .............. 165

Figure 49. Ada's list of possible new groups considering the addition of the inversion center. 
Figure 50. Students' systems for determining symmetry groups before (left) and after (right) considering an inversion center, TE3 .............................................................. 168 Figure 51. Students' systems for determining symmetry groups from TE1 (left) and TE2

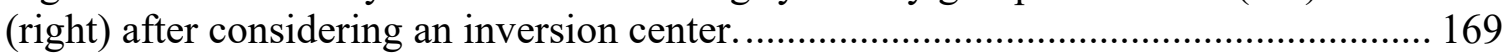
Figure 52. Sample prompt given during Step 6 of the LIT ............................................ 170 Figure 53. Felix's gesture of three-dimensional basis vectors, TE1............................. 173 Figure 54. A typical flowchart given to undergraduates in inorganic chemistry............ 180 Figure 55. The student's final model for classifying chemically important symmetry

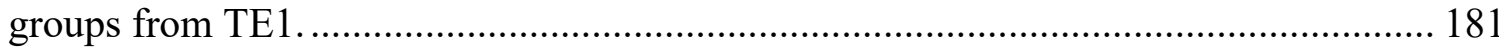

Figure 56. The initial prompt given to participants in TE2 ......................................... 193

Figure 57. Arthur and Stu's Cayley table for the symmetries of water........................... 194

Figure 58. Stu's visual comparison of ammonia and ethane.......................................... 198

Figure 59. Arthur and Stu's geometric representations for the symmetries of ethane.... 202

Figure 60. The symmetries of ammonia and boric acid, respectively. ........................... 204

Figure 61. Investigatory prompt into student thinking on direct products 1 ................ 206

Figure 62. Investigatory prompt into student thinking on direct products 2................. 211

Figure 63. Arthur and Stu's mapping from $\mathrm{D}_{6}$ to " $\mathrm{C}_{3} \mathrm{x} \mathbb{Z}_{2}$ "......................................... 215

Figure 64. Supplemental task to support Arthur and Stu's thinking about isomorphisms 1

Figure 65. Arthur and Stu's initial identification of six elements in ethane to build a

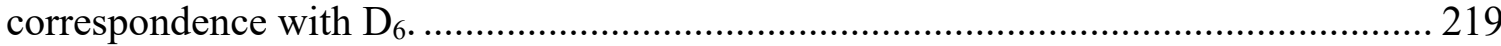
Figure 66. Arthur and Stu's explicit correspondence between six symmetries of ethane

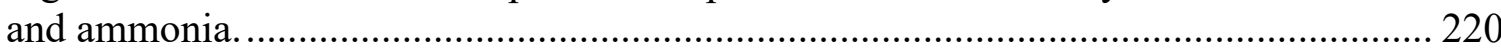
Figure 67. Supplemental task to support Arthur and Stu's thinking about isomorphisms 2

Figure 68. Arthur and Stu's element-wise mapping from the mystery table to ammonia;

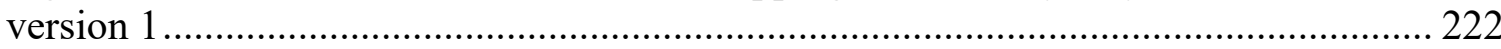

Figure 69. Stu's written work associated with identifying the mystery table as $\mathrm{D}_{6} \ldots \ldots . .222$ Figure 70. Arthur and Stu's element-wise mapping from the mystery table to ammonia;

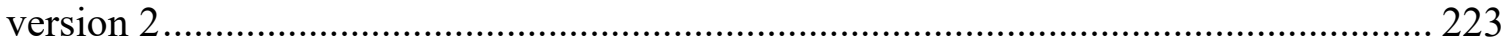

Figure 71. Evidence of Arthur and Stu establishing an isomorphism from the mystery

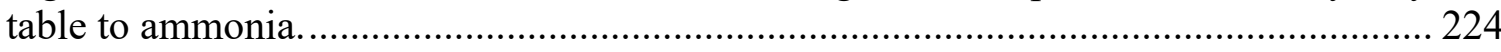
Figure 72. Supplemental task to support Arthur and Stu's thinking about isomorphisms 3

Figure 73. Stu's set notation description of $\mathrm{D}_{6} \times \mathbb{Z}_{2}$ and accompanying Cayley table.... 227

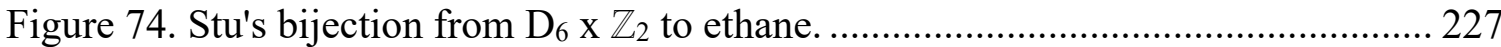
Figure 75. A sample of Arthur and Stu's written work associated with trying to establish

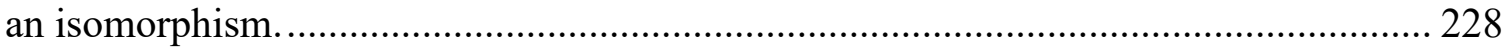
Figure 76. Supplemental task to support Arthur and Stu's thinking about isomorphisms 4

Figure 77. Arthur and Stu's renaming of elements of ethane to better represent the direct

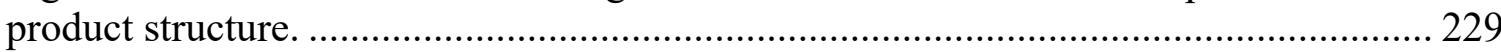
Figure 78. Arthur and Stu's Cayley tables for the symmetries of ethane (left) and $\mathrm{D}_{6} \times \mathbb{Z}_{2}$

Figure 79. Stu's written work associated with trying to untangle an isomorphism ........ 232 
Figure 80. Supplemental task to support Arthur and Stu's thinking about isomorphisms 6

Figure 81. Ada and Sophie's group presentations for water (left), ammonia (center), and

ethane (right). 240

Figure 82. Ada and Sophie's initial model for identifying the symmetry group for a given molecule. 241

Figure 83. Additional molecules given to Ada and Sophie, and the group presentations they produced for each......

Figure 84. Ada and Sophie's "score cards" for each molecule containing its symmetry

group presentation.

Figure 85. Ada and Sophie's sorting of groups into group types by existence of symmetries.

Figure 86. Ada and Sophie's group names............................................................... 246 Figure 87. Ada and Sophie's model for identifying symmetry groups incorporating their new group names. 
Chapter 1: Introduction

The use of group theory, in particular the study of symmetry groups has become an essential tool for chemists when interpreting experimental data and predicting the macroscopic properties of materials based on molecular structure. An understanding of molecular symmetry is necessary from a quantum mechanical standpoint as well because molecular wave functions must conform to the symmetry of the equilibrium nuclear framework of the molecule. The mathematical consequences of symmetry have profound implications for many important chemical applications, such as: electron configurations, molecular orbital theory, vibrational and rotational motion, optical and NMR spectroscopy. Therefore, as Cotton and Wilkerson (1988) explain, 'from a knowledge of symmetry alone it is often possible to reach useful qualitative conclusions about molecular structure and to draw inferences from spectra about molecular structures' (p.28). Nowadays, the use of symmetry to describe molecular structure is widely accepted in the chemistry community, so much so that it is commonly introduced and invoked throughout undergraduate courses in organic, inorganic, and physical chemistry. For example, the symbol $C_{2 v}$ conveys precise structural information to a chemist about a given molecule that could otherwise require long verbal descriptions (Cotton \& Wilkinson, 1988). Because of this utility, the use of symmetry notation in chemistry has become commonplace in the research literature. Therefore, knowledge of the basic concepts, conventions, and symbols is necessary in order to read and appreciate the findings reported in many contemporary research papers.

Chemists use a particular set of groups, known as point groups. The set of elements for a point group is a set of symmetry operations (transformations which return the 
molecule to itself while preserving distances) where each included operation leaves a specific point (or set of points) of a molecule unchanged when applied to a threedimensional molecular structure. This set of symmetries is then paired with the binary operation of composition. There are only a relatively small number of symmetry groups that can occur, limited by both mathematics (possible symmetries in three-dimensional space) and chemistry (too many atoms in a single plane will produce too weak a bond to stay stable). The total number of chemically important symmetry groups is bound to 32 (Zeldin, 1966). In summary, group theory is especially powerful for identifying and differentiating molecules based on the valid symmetry operations associated with their shape.

Due to its powerful ability to simplify problems and guide intuition, symmetry is invoked throughout undergraduate courses in chemistry. Group theory specifically is often formally introduced in undergraduate inorganic chemistry courses, where the basic formalism of point groups, symmetry operations, and character tables are discussed. The ACS requires certified graduates (Marek, Raker, Holme, \& Murphy, 2017) in chemistry to have the equivalent of at least one semester of both inorganic and physical chemistry. The Committee on Professional Training (Larive \& Polik, 2008) lists geometries and symmetry point groups as one of the topics in the inorganic chemistry curriculum.

In traditional instruction, both formal mathematical definitions and rich molecular representations are often presented from the perspective of an expert. These definitions and representations are representative of complex concepts that are initially only meaningful to the expert, instead of the novice, the student, who is meant to extract some particular meaning (Gravemeijer \& Doorman, 1999). Renowned mathematician and 
mathematics educator Hans Freudenthal once said, "to be sure, mathematics is a precious treasure-chest of tools, precious that is for those who can put them to good use" (1991, p. 14). Complex and abstract concepts are typically presented in a distilled, ready-made form intended to be easily digestible for students, but in reality, these ideas are more often the result of years, decades, or possibly centuries worth of thoughtful pursuits riddled with preliminary conjectures and false attempts. Too often in traditional instruction the results of the mathematical (and/or chemical) insights of experts are taken as the starting point for student activity, rather than the viewpoint and knowledge base of the student.

In undergraduate inorganic chemistry courses students are often introduced to symmetry groups by first learning to identify symmetry elements, which are physical properties of a structure such as rotational axes or planes of reflection. The students are then taught to navigate a flowchart which provides them with a classification system for chemically important point groups that provides the corresponding group of symmetry operations which are motions (rotations, and reflections) performable about the identified symmetry elements. For example, a water molecule, as seen in Figure 1 below has one order 2 rotational axis and two vertical planes and so by using the flowchart in Figure 2 we can conclude that the symmetry group of water is referred to by chemists as $C_{2 v}\left(C_{2 v}\right.$ is isomorphic to $\mathbb{Z}_{2} \times \mathbb{Z}_{2}$ ).

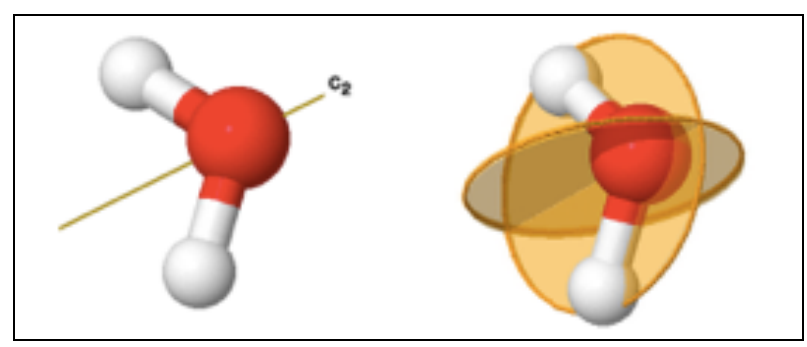


Figure 1. The symmetries of a water molecule; which include an order 2 rotational axis and two vertical planes.

For my dissertation I conducted a design experiment using the instructional design theory of Realistic Mathematics Education, RME. The overarching goal of this experiment is to develop a local instructional theory for the guided reinvention of an algorithm for classification of chemically important symmetry groups, as seen in Figure 2 below. A local instructional theory serves as a generalized roadmap for student reinvention of a particular mathematical concept. This dissertation study both tested and refined a preliminary local instructional theory created from data collected in a pilot study with a pair of mathematics education graduate students. The first paper from this project is written as an existence proof of students' reinvention of a classification system focusing on the findings of the first teaching experiment. Paper 1, found in Chapter 5: Paper 1: Developing an Active Approach to Chemistry Based Group Theory is written for a chemistry education audience and was published in It's Just Math: Research on Students' Understanding of Chemistry and Mathematics, a recent book by the American Chemical Society. The second paper describes the describes the refined local instructional theory informed by the data collected across the entirety of the study. Paper 2 found in Chapter 6: Paper 2: A Local Instructional Theory for the Guided Reinvention of a Classification Algorithm for Chemically Important Symmetry Groups is considered the main deliverable of this study. The LIT, including the tasks used and accompanying rationale, is organized in six steps identified as key in the reinvention process. Each of the six steps are described in terms of students' mathematical activity as they moved through the reinvention process with evidence of students' work from each of the teaching experiments. The third and final paper from this project offers a closer look at 
how the variation in students' mathematical backgrounds informed their mathematical activity. Paper 3 in Chapter 7: Paper 3: Productively Applying and Adapting a LIT for Different Kinds of Mathematical Preparation, describes how I responded to a design challenge that emerged while leading students through the LIT. During the teaching experiments it became clear that students would need a compact representation for symmetry groups to use as outputs for their classification system. Paper 3 describes the partial success and lessons learned from working with students who had limited access to conventional group theory, and the ultimate success when working with students who had no previous group theory exposure. 


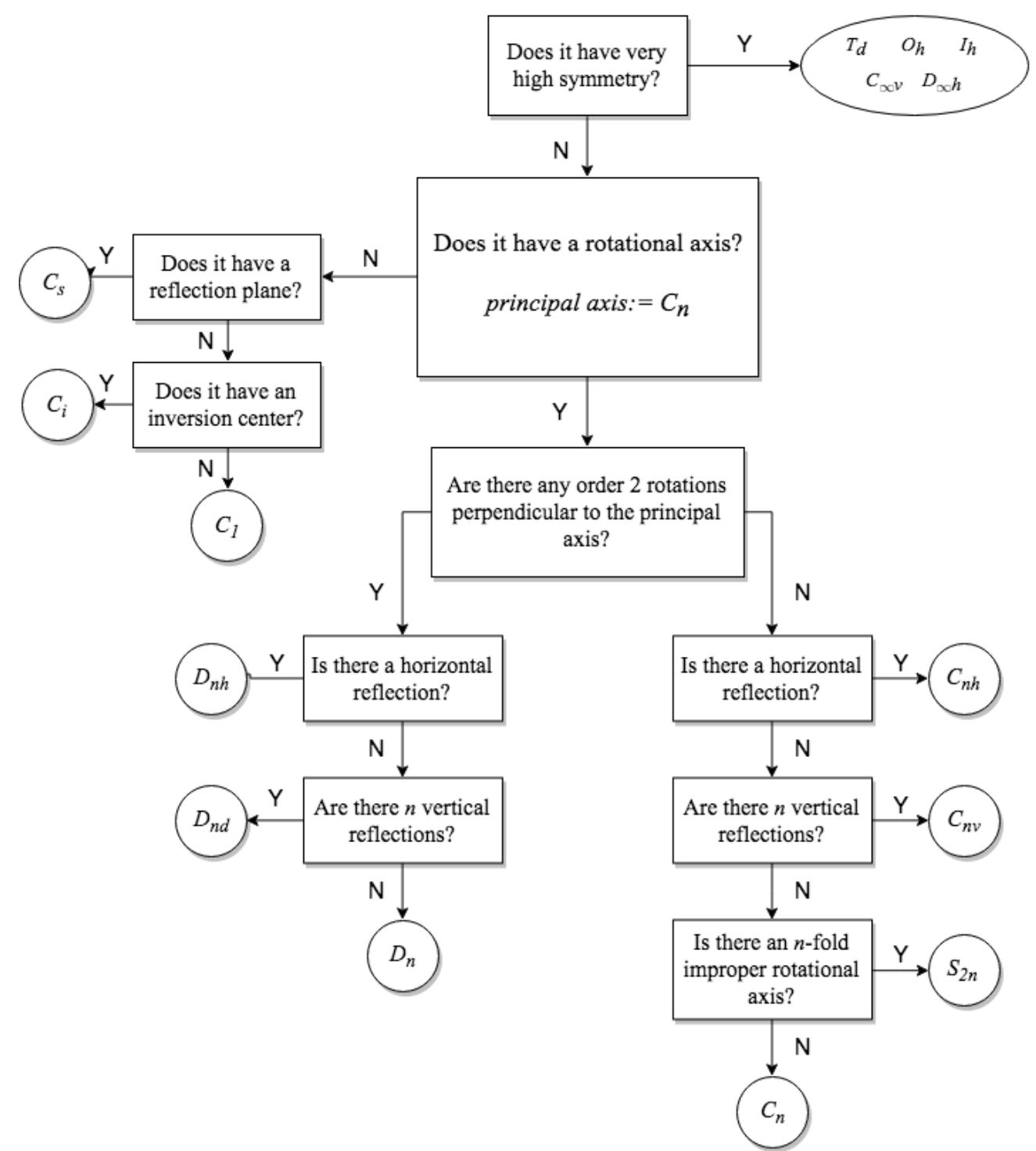

Figure 2. A typical point group classification flowchart given to inorganic chemistry students.

I have chosen the design theory of RME because as a mathematics student I have experienced firsthand the power of being given an opportunity to actually do mathematics. All throughout my undergraduate and master's program I believed that mathematics was a collection of procedures to recall and perform, that all problems had a 'most productive' approach if not a single right answer, and that proofs were something to be regurgitated. It wasn't until the first term of my doctoral program in Dr. Larsen's introduction to abstract algebra course that I was pushed to mathematize, to play around 
with objects and theorems until patterns began to emerge. Beginning with an investigation into the symmetries of an equilateral triangle I finally began to understand what it meant to do math, to mathematize, to trim down reality until only the mathematical relationships remain. This experience not only led to a robust understanding of group theory and abstract algebra, but it changed my fundamental understanding of what it means to do mathematics, and also who could be a mathematician. Throughout my undergraduate and masters, I suffered from severe impostor syndrome; never quite feeling like I was smart enough to be a real mathematician, like I was always just missing something. However, by finally being given an explicit opportunity to mathematize I realized the feeling of not belonging was only because I didn't actually understand what I was meant to do. Through this project I hope to create opportunities for more students to engage in the human activity of doing mathematics, so that they too may gain a deeper understanding and appreciation for this amazing subject.

\subsection{Research Questions}

For my dissertation study, I proposed an instructional design project where the overall goal was to create a local instructional theory for reinventing a classification algorithm, therefore my main research question is the following:

\section{How can students be supported in reinventing an algorithm for the classification of chemically important symmetry groups?}


As students engage in the reinvention process, I will also be focusing on the following research questions which support the main research question.

Sub-research questions include the following:

- What kinds of student strategies and ways of thinking anticipate the formal mathematics necessary to classify symmetry groups?

Answering this question provided support for the LIT, since a lot of the LIT is about moving the informal strategies and student approaches towards more formal mathematical activity, this question helps to identify the informal strategies that can be evoked about built upon. Therefore, the answer to this question is the student strategies and ways of thinking that looked to me as the researcher like the kinds of approaches I wanted them to eventually model.

- As students move through the reinventing process, how is their thinking about symmetry impacted?

The efforts to answer this question also helped provide a way to identify critical junctions in the LIT. The answer to this question looked like some indication that the students' conception of symmetry had shifted in some way as they engage with specific tasks. For example, the evolution of the pilot students' definition of symmetry as they engaged with various molecules is indicative of a change in their conception of symmetry from one that 
indicated an operation or motion conception of symmetry to that of an element or physical property.

- How do students' ways of thinking about symmetry impact their mathematical activity as they reinvent an algorithm for symmetry group classification when starting with their own activity and informal approaches?

Addressing this question helped provide descriptions of student thinking and how it evolved as students completed the various tasks in the LIT. It was also used as rationale for both the tasks and the sequence of tasks in the LIT. The answer to this question looks similar to the 2019 JMM presentation I just gave, What is a rotation? Mapping student's evolving descriptions of 3-dimensional symmetries. In the presentation I talked about how the student's definition of symmetry evolved (as described above) and that this evolution also corresponded with the student's evolution in mathematical activity from situational to referential.

- What contexts/tasks can be used to evoke the informal student strategies and knowledge that anticipate a successful classification system?

This question helped provide rationale for both the tasks in the LIT and how the tasks are sequenced. The 'answer' to this question came in the form of an explanation for why these task evoked successful student engagement with the context and tasks that make up 
the preliminary LIT. Also, any new tasks or contexts that come up during the teaching experiments that weren't present in the pilot that support student success were then incorporated into the revised LIT.

- What kinds of mathematical activity can promote the evolution of the students' informal knowledge and strategies into more powerful ways of thinking, symbolizing, and reasoning?

Efforts to answer this question helped provide support for the various tasks in the LIT. More specifically, the answer to this is a set of tasks and strategies for turning the students' informal ideas into a formal classification system. The goal of each task will be to get students to engage in the task through a particular mathematical activity that helps to promote the evolution of their mathematical thinking and behavior. This question helped to identify the types of mathematical activities that make up these goals. 
Chapter 2: Literature Review

The following literature review will draw on literature from both mathematics education and chemistry education. I will start with an overview of the mathematics education literature on abstract algebra in general, and undergraduate mathematics student thinking related to symmetries and symmetry groups in particular. I will present an argument for introducing abstract algebra by investigating symmetries, along with a survey of various evidence-based instructional innovations within undergraduate abstract algebra which leverage the study of symmetry groups. Then, I will turn my focus to research concerning student thinking related to symmetries from the chemistry education literature. Lastly, I will present various instructional innovations and student supports for learning symmetry identification and point group classification from the chemistry education community.

\section{1 - Mathematics Education Literature on Abstract Algebra}

Abstract algebra has long been considered a challenging course (Leron \& Dubinsky, 1995). The content is abstract, axiomatic, and complex (Melhuish, 2015), where many of the fundamental concepts such as isomorphism require a complex coordination of many ideas (Leron, Hazzan, \& Zazkis, 1995). Furthermore it is also a course in which students are often learning how to prove, "for most undergraduates this course is one of their earliest experiences in coping with the difficult notions of mathematical abstraction and formal proof" (Weber \& Larsen, 2008, p. 139). This has led the majority of research on the teaching and learning of undergraduate abstract algebra to fall into one of two categories: student misconceptions and instructional innovations (Weber \& Larsen, 
2008). In this portion of the literature review, I will offer an overview of research on student thinking in abstract algebra in general spanning over the last quarter century, highlighting student thinking of symmetries and symmetry groups level in particular. I will also discuss research on instructional innovations in abstract algebra by first outlining the argument for teaching abstract algebra with a focus on symmetry groups, and then describing various instructional innovations which leverage symmetry groups.

\subsection{1 - Student Thinking in Abstract Algebra}

The small body of existing literature related to student thinking in abstract algebra unanimously documents the difficulty of complete conceptual understandings of various topics (Melhuish, 2015). Thus, the literature born from the efforts to understand student thinking in the context of abstract algebra often highlights student difficulties and misunderstandings. Much of the early literature was produced by Dubinsky and colleagues exploring how students developed an understanding of the major concepts of group theory using the APOS (Action, Process, Object, Schema) framework (Dubinsky, Dautermann, Leron, \& Zazkis, 1994). Dubinsky et al. summarize the underlying theoretical perspective associated with this framework:

The essence of our theoretical perspective is that an individual, disequilibrated by a perceived problem situation in a particular social context, will attempt to reequilibrate by assimilating the situation to existing schemas available to her or him, or if necessary, use reflective abstraction to reconstruct those schemes at a higher level of sophistication (p. 269).

APOS begins with a genetic decomposition, built from the perspective of an expert, which is a developmental sequence students may use to make sense of a particular mathematical concept (Dubinsky et al., 1994). The levels of each genetic decomposition 
fall into the categories, action, process, object, and schema. Dubinsky et al. define an action as "any repeatable physical or mental manipulation that transforms objects in some way" (p. 270). Once the total action has been interiorized where it can take place entirely in the mind of the individual as a whole, without necessarily running through all of the specific steps the action is said to have become a process. When a process has been encapsulated such that it become possible for a process to be transformed by some action or coordinated with other processes such that a new process is formed the process is said to have been encapsulated into an object. Dubinsky et al. note that there are many mathematical situations in which it is essential to be able to shift from an object back to a process and so one of the tenants of the APOS theory is that this can be done through a process of de-encapsulating the object back to the process which was encapsulated in the first place. Lastly, when a student thematizes a coherent set of process and objects into an identifiable collection that can be used together, it is said to be a schema.

Over the next several years, Dubinsky and his colleagues created genetic breakdowns to investigate concepts beyond their initial investigation into group and subgroup, coset and normality (Dubinsky et al., 1994) using the APOS framework. This includes genetic decompositions for binary operation (Brown, DeVries, Dubinsky, \& Thomas, 1997), coset multiplication, normality, and quotient groups (Asiala, Dubinsky, Mathews, Morics, \& Oktaç, 1997), and symmetries and permutations (Asiala, Kleiman, Brown, \& Mathews, 1998). (The genetic decomposition of symmetries and permutations will be discussed in further detail in the next section of this chapter.) Consider the following genetic decomposition of the group concept described by Dubinsky et al. (1994) as an example; in which they claim that the very first phase of learning the group concept 
similar to an action conception is the conceptualization of a group primarily in terms of its elements, that is, as a set. Students then form a process of associating these objects with others via the binary operation. Eventually, the students may encapsulate the process into an object which, for them, represents the group in question. In each of the studies, the researchers analyzed student work on exams and interviewed students to work towards validating their framework. By in large, their analysis found evidence that the students were working at the various levels of sophistication.

Hazzan's (1999) seminal paper on reducing abstraction argued that a student's reduction in abstraction such as the use of processes over objects did not necessarily equate to a misunderstanding or even a lack of understanding. Rather Hazzan explained that, "the mental process of reducing abstraction level indicated that students find ways to cope with new concepts they learn. They make these concepts mentally accessible, so that they would be able to think with them and handle them cognitively" $(1999$, p. 75$)$. By interviewing undergraduate students and analyzing written questionnaires, Hazzan developed a theoretical framework for ways in which students reduced the level of abstraction while learning abstract algebra concepts. The framework identified three such student methods, including making the unfamiliar familiar, the use of first-person language and a tendency to work with canonical examples to navigate the process-object duality, and lastly by reducing the degree of complexity of a concept of thought.

The first method of making the unfamiliar familiar in an abstract algebra context is tied to the interpretation of abstraction, not as an inherent property of a concept or object, but rather as a property of a person's relationship to an object (Wilensky, 1991). In short, the closer a person is to an object and the more connections they have formed to it, the 
more concrete (and the less abstract) they feel to it. Hazzan observed students basing their arguments on mathematical entities they were more familiar with such as numbers and number operations instead of abstract groups. As students encountered new objects abstractly some personalised the formal expressions and logical arguments by using first person language. For example, when working with quantified expressions students would often replace 'there exists' with 'I can find' (Hazzan, 1999, p. 80). Students also tended to work with canonical examples where their first reaction is to carry out a well known procedure instead of using theoretical knowledge. For example, Hazzan describes a student calculating all six cosets of a subgroup $\{1,2,4\}$ in $\mathbb{Z}>\{0\}$ after clearly describing the fact that cosets divide the set into disjoint equivalence classes. Lastly, students reduced the level of abstraction by reducing the complexity of an object such as dealing with only an element in a set instead of with the set as a whole. Overall, Hazzan's work shows that students may work at various levels in a problem-solving context as they make sense of a problem and develop intuition to their advantage.

In addition to investigating student thinking of specific concepts in abstract algebra there is a growing body of literature which looks at student's coordination between various topics. Much of this work highlights the unfortunate struggles that students have coordinating concepts in abstract algebra, including coordinating simpler concepts into more complex concepts, coordinating multiple interpretations of the same concept, and differentiating between concepts. Leron, Hazzan, and Zazkis (1995) found that for students in their first abstract algebra course, "isomorphism is a complex and compound concept, composed of and connected to many other concepts, which in themselves may be only partially understood" (p. 53). Nardi's (2000) work focusing on student 
understanding of order and the first isomorphism theorem, in which Nardi described as "a container of compressed conceptual difficulties" students struggled at nearly every stage of proving the theorem from notation (the meaning of $\sim$ ) to the definition of a kernel. Hazzan \& Leron (1996) reported on multiple ways in which students mis-use Lagrange's theorem and its converse.

Zazkis, Dubinsky, \& Dautermann, (1996) observed that when investigating $\mathbf{D}_{4}$ some students took a visual approach: using a square, which others took an analytic approach: writing out all possible permutations of the vertices. While Zazkis et al. argued for flexibility between visual and analytic few students showed the flexibility to move between the concrete and the abstract. Lastly there is also evidence that students struggle with differentiating between even fundamental concepts such as associativity and commutativity. Larsen (2010b) observed student's confusion over the idea of 'order' when calculating combinations of symmetries. When discussing the importance of the order in which the students should compute a combination of symmetries the pair conflated the idea of commutativity (relating to the order in which the symmetries are performed) with associativity (relating to the order in which the symmetries are calculated i.e. regrouping). As the symmetries at hand were those of an equilateral triangle as for commutativity order definitely matters since the group $\mathbf{D}_{\mathbf{6}}$ is non-abelian, however since $\mathbf{D}_{6}$ is in fact a group therefore associative and so regrouping is allowed.

It is important to note that most studies involving student thinking in abstract algebra are conducted with a nonrepresentative sample and not replicated. In a recent effort to gain a deeper insight into student thinking of abstract algebra and challenge this paradigm, Melhuish (2015) designed a large-scale study evaluating student conceptions 
in group theory by surveying a nationally representative sample of students. The findings of this large-scale study both challenged and reinforced the results of well recognized research by replicating some of the very questions previously used to build theory around student understanding (Melhuish, 2018, p. 9). The findings also helped to inform the creation and validation of a group theory concept inventory. Building off much of the same existing literature discussed in this literature review, Melhuish (2015) argued that attending to concepts in abstract algebra independent of proof skills is important for several reasons: (1) understanding concepts in group theory is nontrivial, (2) understanding concepts is an essential part of advanced mathematics, and (3) understanding concepts is necessary for a high degree of success in proving (p. 2). It has been claimed that, "mathematics faculty and students generally consider (abstract algebra) to be one of the most troublesome undergraduate subjects" (Dubinsky et al., 1994, p. 268), however Melhuish (2015) posited that student understanding in the course had not been empirically evaluated on a large scale, and that "as a field we needed to develop a coordinated picture of what it means for a student to understand group theory" (p. 35).

Melhuish's (2015) Group Concept Inventory (GCI) is a validated quantitative measure created through both qualitative methods and quantitative methods used to complement qualitative explorations and evaluate larger populations (p. 2). Melhuish began by trying to determine the fundamental concepts in an introductory group theory course by consulting experts, literature, and textbooks in the field. Eventually Melhuish identified eighteen topics vetted by experts of teaching and learning abstract algebra as "important in group theory." Tasks were then designed/selected to gain insight on 
student understanding for each of the topics. Open ended student responses were thematized and reworded into seventeen multiple choice tasks where the items are born from evidence of genuine student thinking. It will be exciting to see the future research based on this powerful tool to further understand student thinking in abstract algebra.

\subsubsection{1 - Student Thinking in Symmetries and Symmetry Groups in Abstract Algebra}

Research literature on student thinking involving symmetries in a group theory context at the undergraduate level is quite sparse and has only been an area of interest for the last two decades. The most well-known and regularly cited work in this small domain is that of Zazkis et al. (1996) and their early work on student coordination of visual and analytic strategies while working with $\mathrm{D}_{4}$. Much of the work since then has been further arguments for an increase in student exposure to symmetry groups as a way to help them gain a deeper understanding to more formal group theory in general and/or evidence that an understanding of symmetry is an asset when reasoning through abstract algebra tasks. In this section I will share various studies in which researchers have claimed that an understanding of symmetry groups can in fact aid in understanding all kinds of topics in abstract algebra and I will also discuss the small body of literature investigating student's cognitive processes related to symmetries in the context of abstract algebra.

In 1996 Zazkis and colleagues published work exploring the interrelationship between visualization and analysis in terms of student thinking. In a response to the current research on student visual and analytic thinking in mathematics whose focus was on individual preferences or learning categories Zazkis et al. instead discussed these different ways of thinking in terms of strategies, approaches, and experiences. Also, 
instead of using a dichotomy between the two ways of thinking Zazkis et al. posited that the situation may be more complex and turned their investigation towards the relation between the two.

Zazkis et al. (1996) provided their own definitions for both visual and analytic thinking strategies along with student data to help illustrate. Below are their working definitions which were used to frame their work:

Definition: Visualization is an act in which an individual establishes a strong connection between an internal construct and something to which access is gained through the senses. Such a connection can be made in either of two directions. An act of visualization may consist of any mental construction of objects or processes that an individual associates with objects or events perceived by her or him as external. Alternatively, an act of visualization may consist of the construction, on some external medium such as paper, chalkboard or computer screen, or objects or events that the individual identifies with object(s) or process(es) in her or his mind. (p. 441)

Definition: An act of analysis or analytic thinking is any mental manipulation of objects or processes with or without the aid of symbols. (p. 442)

After having worked with the symmetry group $D_{4}$, the symmetries of a square, throughout the term and being exposed to both analytic and visual examples several students participated in interviews. In the interviews students were asked to list the elements of the group, and calculate the products, according to group operation, of pairs of elements (Zazkis, et al., 1996, p. 435). Zazkis et al. posited that when considering the group $D_{4}$ both visual and analytic processes represent roughly the same level of mathematical sophistication and that the task is complex enough to bring out distinctions in students' understanding. Throughout their interviews Zazkis et al. found the presence of both analytic and visual components in almost every student response. This further 
supported their assumption that, "it may not make much sense to attempt to classify an individual as either a visualizer or an analyzer" (p. 444).

The visual solution most students employed throughout the interviews generally involved the analytic act of labeling the square and using this frame of references to determine final positions as results of various symmetries. Whereas the students' analytic strategies involved interpreting the elements of $D_{4}$ as permutations, which Zazkis et al. claims "is a process that requires at least some connection to the square as a visual element" (1996, p. 444). However, Zazkis et al. also noted that even when both strategies appear to be available to the student they can often have difficulty making the connections between them. Students who could seem to mix, harmonize, and synthesis the two strategies usually also had a more mature understanding of the problem. Zazkis et al. went on to describe how visual approaches are enriched by analytic thinking and how analytic approaches benefit from visualization with the use of the Visualization/Analysis (VA) model in Figure 3 below. The VA model describes a sort of continuum between the two approaches and shows how their relationship is a "symbiosis rather than a rivalry of opposite pole" (p. 455).

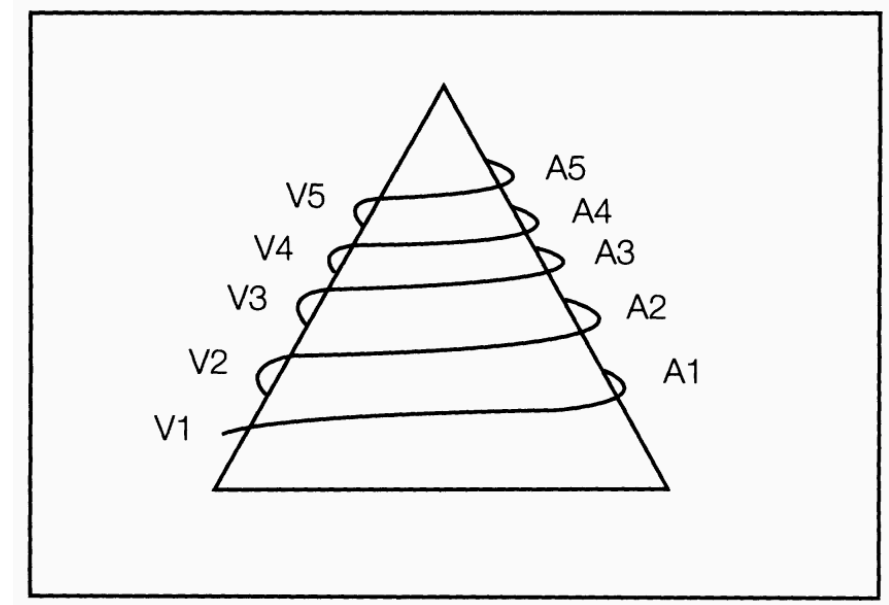

Figure 3. Visualization/Analysis Model (Zazkis et al., 1996, p. 447) 
While Zazkis et al. (1996) made an argument for the benefit of visualization in advanced mathematics, more current research had shown that students themselves also seem to want more access to visualizations in abstract algebra. As part of a much larger dissertation study Ioannou and Nardi (2009) observed students' apparently diminishing engagement over a ten week abstract algebra course. One of the factors leading to disengagements was students' relationships with the subject matter, in particular the effect that the abstract, not easily visualized nature of abstract algebra had on their relationship with the topic. By offering the following student comments (2009, p. 39):

"Yeah, and like... in the - in the proofs as well, it's like - oh, but the that means this, and it's just trying to understand just why that means that, and because I can't see it in my head, and I can't visualize it, it just I don't see why they're so you know - like it goes and therefore this, and I'm just like - but why?" [KL2]

"I find it hard to picture, I find it hard to... understand what's really happening... what's meant by certain things. I thought when it first started, oh this is going to be ok, but then it just was like - with ideals and stuff, it was just really confusing, I was like, oh...[...] to be able to picture it in my head." [LH3]

Ioannou and Nardi shed light on how students' relationships with abstract algebra is affected by the difficulty to visualize thus reinforcing Zazkis et al.'s (1996) claim that constructing appropriate visual imagery can provide crucial support to student meaning making.

Similarly, Almeida (1999) argued that students' understanding of the formal aspects of group theory could be enhanced by a substantial investigation of symmetry. He posited that simple symmetry arguments may have helped students who experienced difficulties while appearing to rely on purely formal methods. Almeida worked with two 
groups of undergraduate mathematics students, one of which had extensive exposure to symmetry theory (lasting 50 hours) referred to as the trainees and the undergraduates who were expected to learn group theory in a formal way starting with axioms. After both groups had learned group theory concepts up to the beginnings of quotient groups and isomorphism theory Almeida looked at differing student approaches to two ideas in group theory; the theorem of two reflections and the inverse of a group element.

The theorem of two reflections "states that the composite of two reflections with intersecting axes is a rotation about the point of intersection" (Almeida, 1999, p. 162). The trainees were introduced to the theorem informally but precisely as seen in Figure 4 below:

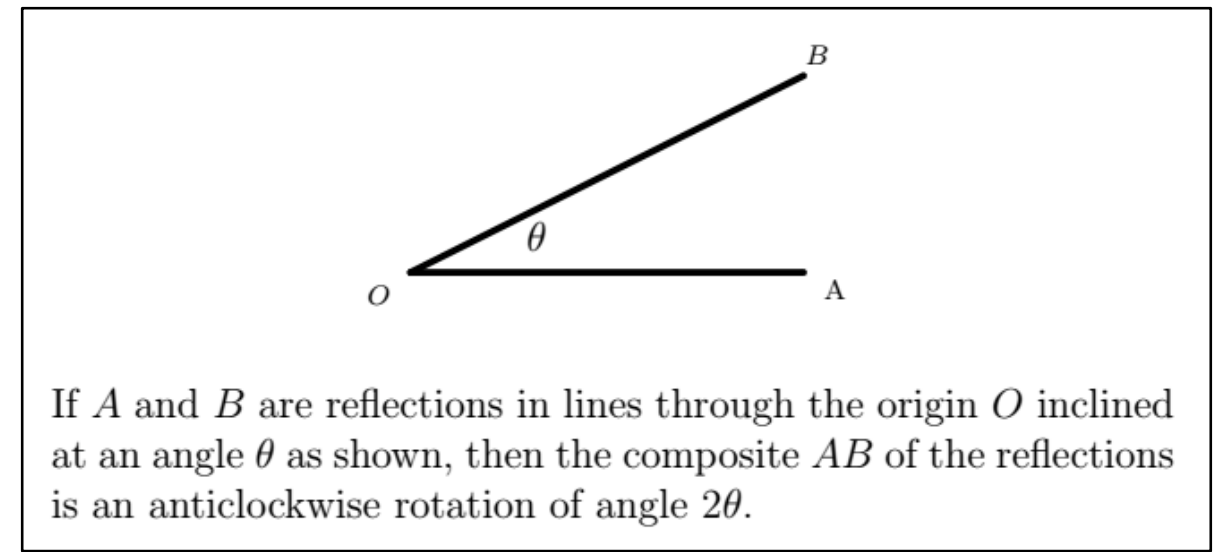

Figure 4. The trainees' introduction to the theorem of two reflections (Almeida, 1999, p. 162).

First a proof using Euclidean geometry was given to the trainees, followed by a more formalized proof using $2 \times 2$ matrices. In contrast, the undergraduate group without the intense symmetry exposure was introduced to the same theorem in the following assignment, seen in Figure 5. 
1. If $A \in O(2)$ show that $\operatorname{det}(A)=1$ or -1 .

2. If $A \in O(2)$, but $A \notin S O(2)$, show that

$$
A=S_{\theta}=\left(\begin{array}{cc}
\cos \theta & \sin \theta \\
\sin \theta & -\cos \theta
\end{array}\right)
$$

for some $\theta \in \mathbb{R}$. Show that this matrix represents a reflection about a line through the origin. Identify the axis of the reflection.

3. Let

$$
R_{\theta}=\left(\begin{array}{cc}
\cos \theta & -\sin \theta \\
\sin \theta & \cos \theta
\end{array}\right)
$$

be the matrix representing a rotation through an angle $\theta$ about the origin. Recall that $R_{\theta} R_{\phi}=R_{\theta+\phi}$ and $R_{\theta}^{-1}=R_{-\theta}$. Find similar expressions for $R_{\theta} S_{\phi}, S_{\theta} R_{\phi}, S_{\phi} S_{\theta}$, and $S_{\theta}^{-1}$.

Figure 5. The undergraduates' introduction to the theorem of two reflections (Almeida, 1999, p. 162-163).

The undergraduates were directed to use $2 \times 2$ matrix representations of rotations and reflections to express the theorem in symbols. Both groups were then asked the following prompt, (Almeida, 1999, p. 163):

Problem: Show that there are matrices $A$ and $B$ in $O(2)$ such that $A^{2}=B^{2}=I$ and $A B$ has infinite order.

After examining the efforts put forth by both sets of students, Almeida claimed that, "it was perhaps not surprising that no student (in the undergraduate group) applied the theorem of two reflections in its generic form to deduce the result" (1999, p. 163).

Almeida (1999) also investigated students' conceptual understanding of the inverse of a group element, by looking at student attempts to the following problem (Almeida, 1999, p. 164):

Problem: Show that the number of elements $g$ in a finite group $G$ with $g \neq g^{-1}$ is even. He observed that most of the undergraduate students viewed the problem in the abstract domain and did not leverage any generic examples. He claimed that most students 
preferred to use/misuse the formal rules of mathematical logic. He conjectured that the students' concept image of inverse had been "impoverished by a lack of generic examples and/or appeals to diagrams" $(1999$, p. 164). While Almeida does a nice job of highlighting very specific instances where a deeper understanding of symmetry and symmetry groups may be helpful, he fails to include any data related to the trainees' approaches and/or any indication of how successful they were with leveraging their understanding of symmetry.

More recently, Dubinsky, Henderson, and Nosrati ( 2017) have provided an example of the "ensuing dance between geometric visualization and formal analytic thinking" ( $p$. 53). In an effort to extend intuitive geometric discussions in the plane to discussions of isometries and edge-preserving permutations of cubes in 3-space Dubinsky et al. offer a number of proofs to theorems involving hypercubes. Their detailed examples show a synthesis of analytic thinking and geometric visualizations throughout the development of the proofs and they hope that these will be helpful for students who are learning to understand and make proofs. While they don't provide any student data, they do feel that their arguments illustrate one way to combine visual and analytic thinking as recommended by Zazkis st al. (1996).

Asiala et al. (1998) used APOS theory to design genetic decompositions, or theoretical frameworks which were used for understanding the mental constructions made by students when learning about both permutations and symmetries. The framework for symmetries was quite narrowly designed, and only considering student thinking on symmetries of regular polygons such as equilateral triangles or squares. Asiala et al. claimed that since symmetries can be conceptualized as functions, the following schema 
for symmetries would be 'close to, but not exactly' an application of an individual's function schema (1998, p. 18-19):

Action. An action conception of symmetry might be indicated by a subject's need to physically manipulate a model of a regular polygon in order to consider each particular symmetry.

Process. A process conception of symmetry might be indicated by the subject's ability to imagine performing the symmetry without actually doing it. This might be done by forming a mental picture of a regular polygon and consciously rotating or flipping it.

Object. An object conception of symmetry might be indicated by the subject's ability to conceive of forming the set of all symmetries of a polygon. Another possible indication of an object conception is being able to think about the composition of two symmetries as a function that inputs two symmetries and outputs a symmetry.

Asiala and colleagues (1998) used their framework to classify student understanding of symmetries by their response to the same prompt used in Zazkis et al. (1996); "Let $D_{4}$ be the group of symmetries of a square. Write out the elements of $D_{4}$ " (p.25). The question provided a basis for the questions in the first of two interviews. Asiala et al. found each of the three types of conceptions of symmetries as per their framework, and in total they found that $20 \%$ of the students were 'limited' to an action conception, $40 \%$ held a process conception, and 40\% held both a process and an object conception (1998, p. 38). While the APOS framework for symmetries may be a start to investigating student thinking on symmetries it does have limitations. Asiala et al. describe one such issue (1998, p. 40),

Our genetic decomposition for permutation (symmetries) was based on a previously established genetic decomposition for the concept of function. It was useful in interpreting the data of this study, but in fact, there were few direct references to the concept of function by the students interviewed. The extent to which students explicitly interpret permutations (symmetries) as functions is an open question at this point. 
Lastly, a study by Portnoy, Grundmeier, \& Graham (2006) explored student's view of geometric objects as they explored relationships between transformational geometry and linear algebra, including groups of isometries. Working with preservice teachers the researchers investigated how the students viewed transformations; as process or as objects and how they viewed the objects of transformations; as conceived or perceived ( $\mathrm{p}$. 201). The data presented suggested that the students viewed the transformations as primarily operational processes as opposed to objects. Students often spoke of performing the transformation on the figure and all of the participants found it difficult to prove that the set of Euclidean transformations formed a group. Portnoy et al. suggested that the students' inability to see the transformations as objects aided in this difficulty.

As the students engaged with the tasks the researchers (Portnoy et al., 2006) also investigated how students were viewing these objects, as either perceived or conceived. Tall, Thomas, Davis, Gray, and Simpson (2000) provide an explanation of the difference between students' perceived geometric objects as drawings, etc. and their conceived geometric objects as those in their mind's eye which are perfect entities with no realworld equivalent. The evidence found by Portnoy et al. suggested that the preservice teachers were working with perceived geometric objects as they often made reference to specific figure instead of arguing about the inherent properties of the figures more generally.

\subsection{2 - An Argument for Teaching Abstract Algebra with Symmetry Groups}

Debate over the best overall approach to teaching group theory, in particular how students should be introduced to the subject has been the subject of a rather lively debate 
within the mathematics education literature (Portnoy, Grundmeier, \& Graham, 2006). Nearly 50 years ago Hans Freudenthal (1973) argued for teaching abstract algebra, in particular group theory, by first investigating automorphism of sets onto themselves. Freudenthal argued that this was a natural way for students to approach group theory that built off their own intuitions and informal approaches. He posited this approach against the standard approach to instruction where one begins by defining an abstract group and then proceeds by proving general theory. Freudenthal claimed that mathematics does not develop from general to particular in the minds of individuals, nor historically where definitions and theorems only appear at the end of mathematical exploration. Furthermore, as a research mathematician Freudenthal saw little use in ready-made mathematics, and characterized this approach to instruction as an anti-didactical inversion (Gravemeijer \& Terwel, 2000). Freudenthal sensed, "things were upside down if one started by teaching the result of an activity rather than by teaching the activity itself" (Gravemeijer \& Terwel, 2000, p. 780).

Freudenthal (1973) argued that "groups are important because they arise from structures as systems of automorphism of those structures" (p. 109). For example, the group of symmetries of a salt crystal, that is, a lattice with $\mathrm{Na}$ and $\mathrm{Cl}$ atoms alternating in the lattice corners can be built from considering all the possible translations, rotations, and reflections which carry $\mathrm{Na}$ atoms into $\mathrm{Na}$ atoms and $\mathrm{Cl}$ atoms into $\mathrm{Cl}$ atoms preserving the structure. In this case, the automorphisms of the structure form a group with composition as the group operation. Freudenthal claimed that, introducing groups as automorphism groups of certain structures guarantees that the thing defined is a group; then rather than by an algorithmic verification, this result is obtained in one conceptual 
blow, which is a great advantage. Furthermore, a preference to conceptual over algorithmic approaches is one of the most conspicuous features of what is really modern in modern mathematics (p. 110).

Burn (1996) offered a critical analysis of Dubinsky et al. (1994) (discussed above) in which he argued that the notion of permutation and symmetry should be regarded as the fundamental concepts of group theory. Burn claimed that had Dubinsky and colleagues "initiated their study with permutations and symmetries, the orbits and stabilizers of geometric figures and polynomials would have provided contexts (coinciding with the historical contexts) in which subgroups, cosets and normal subgroups would have stood out as meet for definition" (p. 376). However, Burn does not provide any explicit evidence for this claim; he does note that he had begun a group theory course with "50 hours on geometric symmetry, before offering axioms, which were then immediately valued by the students" (p. 377). Again, no evidence of students' value or understanding of the group axioms was provided. In a response to Burn's critic Dubinsky et al. (1997) argued that perhaps they should have inserted the word "some" in their title to make it “On Learning Some Fundamental Concepts of Group Theory” (p. 251). Dubinsky et al. also point to the lack of research, and therefore literature, investigating how students might be able to come to understand the abstract theory of groups beginning by working with symmetry and permutation. In the next section I will discuss the very literature which has begun to fill this void.

Almeida (1999) explains that the philosophy of undergraduate group theory courses appears to be distributed between two camps: the first are those who build the group axioms on the basis that 'they are the ones satisfied by the groups (of symmetries and 
permutations) one wants to study' and the other believe the converse, that 'introducing group structure first gives a better understandings to symmetries' (p. 160). He argues that this leads to group theory courses starting in one of two directions: beginning with symmetry and building group axioms or beginning with group axioms with just passing references to symmetry. He also notes that there tends to be a preference amongst instructors for the later approach, perhaps due to time concerns. Almeida argued that students' understandings of the formal aspects of group theory would be enhanced by a substantial investigation of symmetry, despite concern for time constraints. After claiming to begin his course with an in-depth study of symmetry lasting 50 hours, Almeida's findings were similar to Burns mostly just claims, and devoid of much actual evidence of student work.

\subsubsection{1 - An Evidence Based Instructional Innovation in Abstract Algebra Leveraging Symmetry Groups}

Investigating the symmetry of shapes has proved to be a productive introduction for students learning abstract algebra. In fact, the investigation of the symmetries of an equilateral triangle has been successfully used as a starting point in the research-based, inquiry oriented abstract algebra course entitled Inquiry Oriented Abstract Algebra (IOLA) (Almeida, 1999). Larsen (2004) was first motivated by the desire to develop "an approach to teaching group theory in which the starting points are meaningful to the students, the ideas develop through the students' own mathematical activity, and the formal definitions of group and isomorphism come from this activity" (p. 11). Through a design experiment consisting of a series of teaching experiments conducted with pairs of students Larsen (2013) developed a LIT which aimed to capitalize on the potential 
Freudenthal (1973) and Burn (1996) described in introducing abstract algebra by working with systems of automorphisms of a structure under composition, aka symmetries.

Through years of design research projects and various scaling up efforts (Larsen, Johnson, \& Bartlo, 2013), Larsen and his team developed an true instructional innovation for a term-long group theory course which begins with an investigation of symmetry. The Inquiry Oriented Abstract Algebra (IOLA) curriculum intended for an introductory abstract algebra course covers groups, subgroups, isomorphisms, quotient groups, and homomorphisms. In the IOAA curriculum students begin by reinventing the group concept via a local instructional theory, best described as a sequence of steps in terms of students' progressive mathematical activity (Gravemeijer, 1998) begins with an investigation of the symmetries of an equilateral triangle. Students are asked to identify, describe, and symbolize the set of symmetries. As the students begin to analyze the symmetries of the triangle the group structure begins to emerge as a model-of the students' mathematical activity. As the students' activity transitions from analyzing combinations of symmetries geometrically to calculating combinations algebraically the rules they develop include axioms featured in the definition of group. Students work together to reduce their list to a minimal set of rules needed to completely determine an operation table for combining pairs of symmetries, at which point the students have transitioned from mathematizing the geometric context to mathematizing their own activity. The reinvention process concludes with students analyzing other groups and defining the group concept in terms of the properties shared by these systems (Larsen, 2013). 


\section{2 - Group Theory in Undergraduate Chemistry}

Due to its powerful ability to simplify problems and guide intuition, symmetry is invoked in undergraduate courses in chemistry. Group theory specifically is often formally introduced in undergraduate inorganic chemistry courses, where the basic formalism of point groups, symmetry operations, and character tables are discussed. These symmetry operations are applied to relatively simple molecules in order to categorize them into their respective point groups. Character tables are also sometimes discussed in physical chemistry courses during units on vibrational spectroscopy. The ACS requires certified graduates in chemistry to have the equivalent of at least one semester of both inorganic and physical chemistry (Marek et al., 2017). The Committee on Professional Training lists geometries and symmetry point groups as one of the topics in the inorganic chemistry curriculum, but does not require that it be taught (Larive \& Polik, 2008).

A recent national survey of inorganic chemists began to shape a landscape of selfreported topics explored in both foundation-level (Raker et al., 2015a) and in-depth coursework (Raker et al., 2015b) in upper division inorganic chemistry. Raker et al. found that $75.4 \%$ of the 317 respondents covered symmetry and group theory (2015a, p. 975 ) in their foundation course with an $18.3 \%$ increase over the last five years in symmetry and group theory coverage. Similarly, $83.8 \%$ of the instructors reported covering symmetry and group theory in their in-depth inorganic chemistry course, with a $19.3 \%$ increase in symmetry and group theory content coverage over the last five years (Raker et al., 2015b). These findings support the notion that symmetry and group theory are very important to the overall knowledge of inorganic chemistry students. 
While there is an argument for why it is important for chemistry students to learn group theory and there is evidence that this importance is intensifying, the literature on the student learning of group theory in chemistry is quite limited. Similar to the mathematics education literature on the student learning and thinking of abstract algebra the chemistry education literature on student thinking and learning of point groups can be described in mostly two categories those related to student thinking related to point groups, and instructional innovations that are argued to have been productive in helping students better understand point groups.

Symmetry is so pervasive in chemistry that is has even been used to help support students in having a deeper understanding of many chemical concepts. McKay and Boone (2001) tested a hypothesis, similar to Almeida's (1999) work described earlier in Section 2.1.1.1, that an early emphasis on symmetry may help students to better understand chemical concepts in subsequent courses and so they expose three different groups of students to symmetry material early in their studies. First-semester general chemistry students were asked to identify basic symmetry elements and operations in the context of molecular structure model, organic students were introduced to symmetry operations as a group and asked to classify molecules into point groups, lastly intermediate inorganic students used symmetry and group theory applications to facilitate the study of molecular orbitals and spectroscopy. Each of the groups of students studied symmetry for at least two weeks and up to a month. Two to four months later the students were asked follow up questions related to their recollection of symmetry theory, any continued use of symmetry theory, enjoyment, usefulness, etc. According to McKay and Boone, "every student felt the symmetry exercise gave a better understanding of 
symmetry and the perception of objects" (p. 1489). Furthermore, all of the students felt the exercise helped them to visualize objects in three dimensions, $31 \%$ stated they had applied symmetry elements and point groups to additional objects in the ensuing month.

\subsection{1 - Chemistry Student's Thinking Related to Symmetries}

Similar to mathematics education, research focusing on symmetry groups within the chemistry education community often highlights student difficulties with learning symmetry theory in chemical contexts. One of the most common student struggles is with visualization. Some argue that when learning group theory, in a chemistry context, logical-visual spatial skills dominate the content to be mastered by students (Habraken, 2004). Research on spatial ability and its ties to intelligence have been studied since the 1800s. Some of the earliest work by Sir Frances Galton (1880) investigated how people differ in their "mental disposition" through the use of mental imagery. At this point mental imagery was defined as, "different degrees of vividness with which different persons have the faculty of recalling familiar scenes under the form of mental pictures, and the peculiarities of the mental visions of different persons" (p.301). Over the following century researchers first established spatial ability and visualization as factors separate from general intelligence and by the second half of the 20th research on spatial abilities had branched off into three different directions, a focus on development of spatial abilities, identification of variance, and the reanalysis of data using common methodological frameworks (Harle \& Towns, 2010).

Two meta-analytic studies (Carroll, 1993; Lohman, 1979) are often cited as when describing the factors of spatial ability. While the taxonomies of the factors of spatial 
ability are not in complete agreement they do have significant overlap and together offer a coherent description of what is being considered as factors of spatial ability by the chemistry education community. Lohman (1979) defined spatial ability as: "the ability to generate, retain, and manipulate abstract visual images. At the most basic level, spatial thinking requires the ability to encode, remember, transform, and match spatial stimuli" (p.352). Lohman further described major factors 1-3 below, of spatial ability and Carroll (1993) later added 4-6 below (as listed by Harle \& Towns, 2010, p.352):

1. Spatial Relations: This factor is composed of tasks that require mental rotation of an object either in plane (2-D) or out of plane (3-D).

2. Spatial Orientation: This factor involves the ability to imagine how an object or array would look from a different perspective by reorienting the observer.

3. Visualizations: This factor is composed tasks that have spatial-figural components such as movement or displacement of parts of the figure and are more complex than relations or orientation tasks.

4. Closure Speed: The ability to identify a partially obscured or vague object without knowing the identity of the object in advance.

5. Flexibility of Closure: The ability to disembed a specific hidden or obscured figure or figures (patterns) in a larger, more complex figure.

6. Perceptual Speed: The speed in finding a unique item in a group or identical items, a specific visual pattern in a visual field, or in accurately comparing one or more patterns when the items or patterns are not obscured.

The ability to generate and recognize drawings of molecules and symbols, and to correctly reason with them is referred to as a part of students 'visualspatial skills' (Harle \& Towns, 2010). Unlike students in mathematics education who tend to investigate symmetry groups of two-dimensional figures (Almeida, 1999; Burn, 1996; Larsen, 2010a) chemistry students are concerned with molecules, all of which are threedimensional. This need for three-dimensional thinking and mental manipulations is believed to be one of the major hurdles for students when learning chemistry in general. When learning about symmetry in particular, students often struggle with both visualizing molecules in three dimensions and also determining the relevant symmetry operations 
(Flint, 2011). “Significant logical-visual spatial skills, including visualization and rotation (i.e. spatial relations), are required to identify symmetry elements and place molecules in point groups. These skills are then partnered with conceptual knowledge to predict vibrational spectra and chirality" (Harle \& Towns, 2010, p. 356). However, it has been shown that even with the use of three-dimensional modeling software and the pervasive use of modeling kits, students still have a difficult time visualizing threedimensional molecular representation of molecular structures (Cooper, Underwood, Hilley, \& Klymkowsky, 2012; Grove et al., 2009; Harle \& Towns, 2010).

Researchers have found that rotation and reflection transformations are particularly troublesome for students. A ( 2001) study by $\mathrm{Wu}$ and colleagues investigating student difficulties in learning chemical representations found that many of the students had difficulties involving mental transformations between two-dimensional (2-D) and threedimensional (3-D) representations. More specifically, the researchers found that many students who could form a 3-D representation of a 2-D image could not mentally rotate it accurately. As for reflections, in separate studies, Tuckey et al. (1991) and Shubbar (1990) found that among students who could correctly interpret depth cues on various representations, few could mentally track how those cues changed as the molecule was rotated about an axis or reflected through a plane. Some researchers claim that improper rotations and inversions, "frequently pose the greatest difficulties for students" (Sein, 2010, p. 827) but fail to provide any data as evidence. Notice that there are only four distinct of symmetries found in point groups, rotations, reflections, improper rotations, and inversions which means each is mentioned somewhere in chemistry education literature as being particularly difficult for students. 
The ability to interpret depth cues and to rotate or reflect a given representation of a molecule is important in student understanding of a wide array of chemical concepts, including group theory. Research has shown that students struggle with the interpretation of complex representations of molecular structures, and then connecting the microscopic structure of a substance to predictions about its macroscopic behavior (Cooper et al., 2012). There are two predominant approaches through to be used by students to solve problems in group theory (Southam \& Lewis, 2013); the first is imagistic reasoning in which students conceptualize the representation, apply an operation, and produce an outcome. This first approach is also an extremely frequent instructional approach. Alternatively, more cognitively demanding approaches include feature-based strategies that may avoid an imagistic route altogether. While experts can often use either or both of these approaches students may not experience the same efficiency (Stieff, 2007). "Without a robust understanding of the underlying ideas that allow the structure-property connection, there is no organizing framework for most of chemistry and students, out of necessity, resort to memorization, and generation of heuristics" (Cooper, Corley, \& Underwood, 2013, pp. 699-700). A student's ability to draw molecular representations and use them to explain physically observable phenomena requires both the development of spatial abilities and an understanding of the chemical content.

While much of the literature concerning student's spatial abilities related to understanding symmetry in three-dimensions highlights student struggles, one study has much more positive results. Process-oriented, guided-inquiry learning (POGIL) curriculums have become quite popular within the chemistry education community with materials available for many topics in chemistry (Moog \& Spencer, 2008). One of the 
main features of a "POGIL classroom" is the role of the teacher, which is different from a more traditional classroom. Rather than lecture on new material to students, the teacher in a POGIL classroom facilitates groups of three or four students and guides them through the new material by actively listening to conversations within groups deciding whether to or how to intervene in the form of guiding questions (Luxford, Crowder, \& Bretz, 2011). A 2013 study looked for any observable differences in a student's abilities and experiences in a POGIL group theory classroom resulting from the student's spatial abilities. The authors used a series of visualization tests to measure student spatial abilities prior to their participation in a POGIL group theory curriculum (described in more detail in Section 2.2.2 below). The students used a variety of strategies using multiple spatial abilities throughout the class and the results showed that there were no observable differences in student performance in the classroom as a consequence of different strategies used to solve the tasks presented (Southam \& Lewis, 2013).

2.2.2 - Instructional Innovations for Chemically Important Group Theory and Point Group Classification

In response to the growing concern that students frequently have difficulty understanding molecular structure beyond two dimensions despite the prevalent use of model kits and modeling software there have been many instructional innovations in chemistry education literature designed to teach students about symmetry and group theory. In this section I will highlight some of these innovations and approaches. The most well researched instructional approach to teaching is a symmetry POGIL activity for inorganic chemistry (Luxford et al., 2011). Process-Oriented Guided Inquiry Learning (POGIL) is one of the most well-known evidence-based curricula in chemistry and is 
used at both the secondary and post-secondary levels (Moog \& Spencer, 2008). POGIL activities are structured using three stages of Novak's learning cycle exploration, concept invention, and application (Luxford et al., 2011). Starting with exploration where students begin connecting prior knowledge supplied in the form of models to new knowledge. The second stage is concept invention when students actively work in groups to articulate their understanding of the patterns and relationships they see in the models they are provided, and lastly students apply the new concept through the use of exercise questions and additional readings.

The primary goal of the symmetry POGIL activity is to expose students to symmetry elements and symmetry operations, and to aid students in the visualization of the symmetry operations (Luxford et al., 2011). Groups of students are provided with molecule kits to help visualize various symmetry elements and operations of both ammonia and trans-dibromotetrachlorocobaltate. After a period of exploration, the students are asked to develop their own definitions and explicate their understanding of various symmetry operations. This approach allows students to explore symmetry elements and operations through student-centered learning rather than traditional lecture. While researchers have found that the students find the creation of definitions of common symmetry terms challenging, POGIL experts reinforce that there are benefits to having students create their own definitions, rather than simply providing them with a standard definition (Luxford et al., 2011). Additional research has been conducted using the POGIL curriculum to look at student's spatial abilities in undergraduate inorganic chemistry (described in Section 2.2.1 above). 
Most of the instructional innovations, activities, and resources currently available to teach chemically important group theory are designed to support student identification of symmetry elements and operations, and to check for student understanding rather than challenging students to develop their own ideas (Luxford et al., 2011). Many of the activities described in the literature utilize some kind of physical model for students to investigate as seen in Figure 6 below. Dynamic paper constructions have been used to assist students' visualization of symmetry operations (Sein, 2010). Flint (2011) describes a collection of three-dimensional models built from over a decade of teaching special topics courses in group theory are used to assist students in their understanding of the structure of symmetry groups. He has also modified various preexisting molecule sets to help students better visualize mirror planes and $C_{2}$ rotational axis perpendicular to the principal axis as seen in Figure 6 below.

Models and manipulatives used to support the student learning of symmetry in chemistry

Dynamic Paper

Constructions

(Sein, 2010, p.

827)
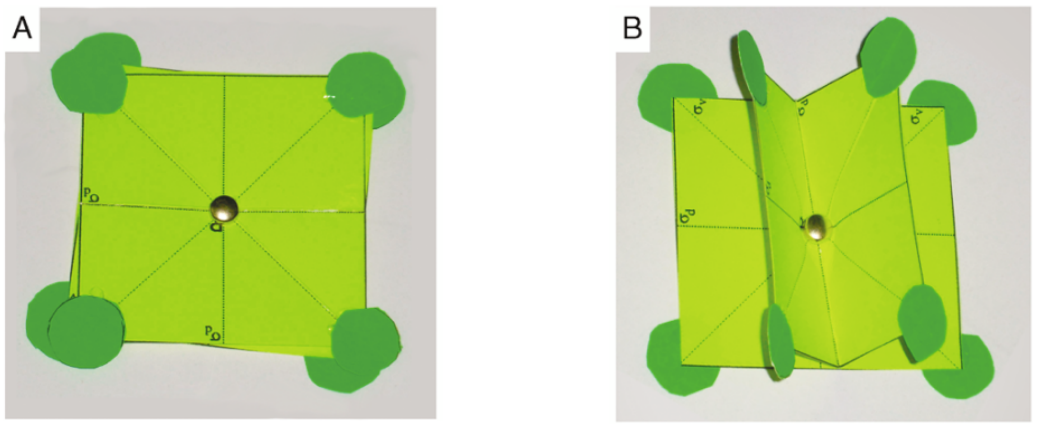


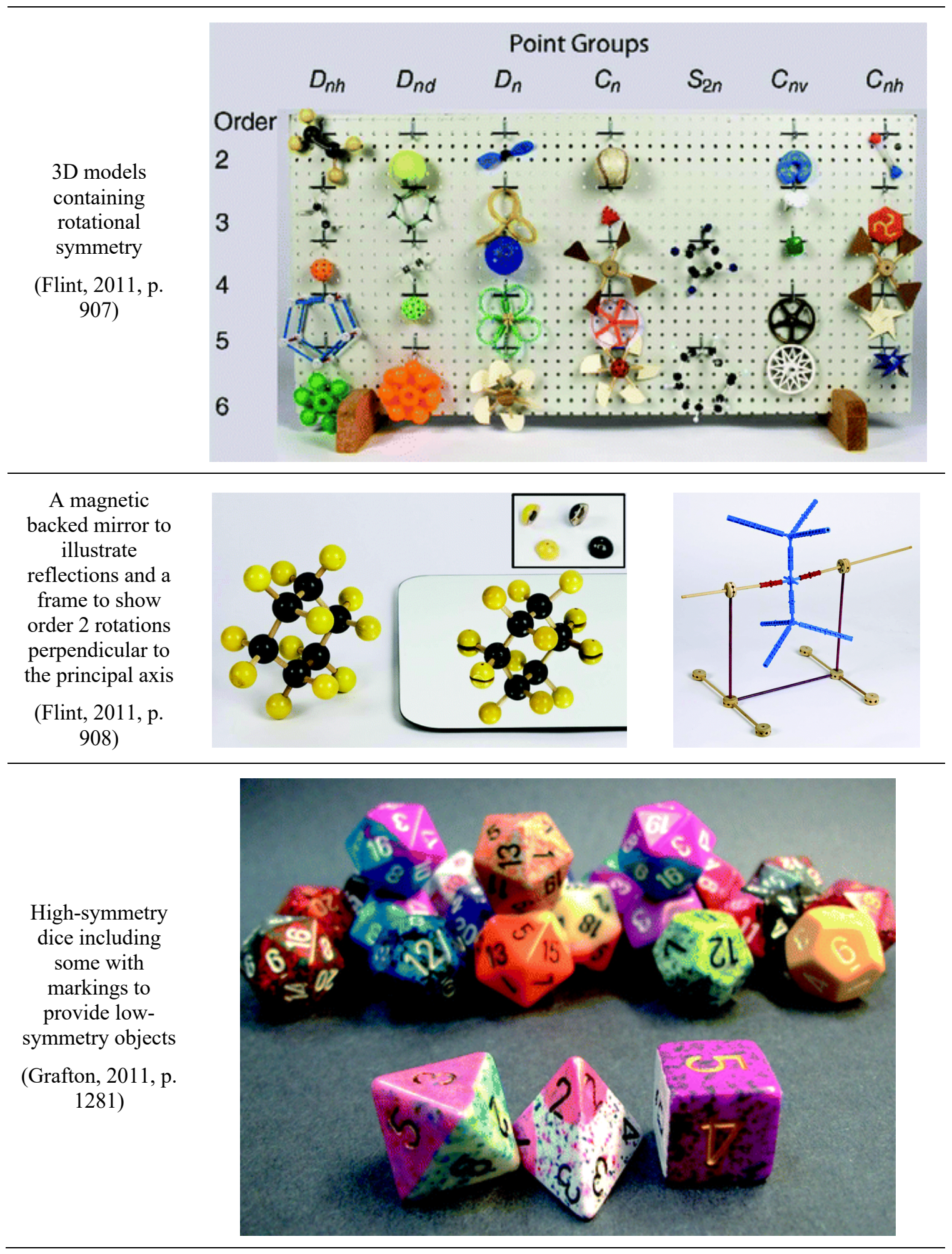




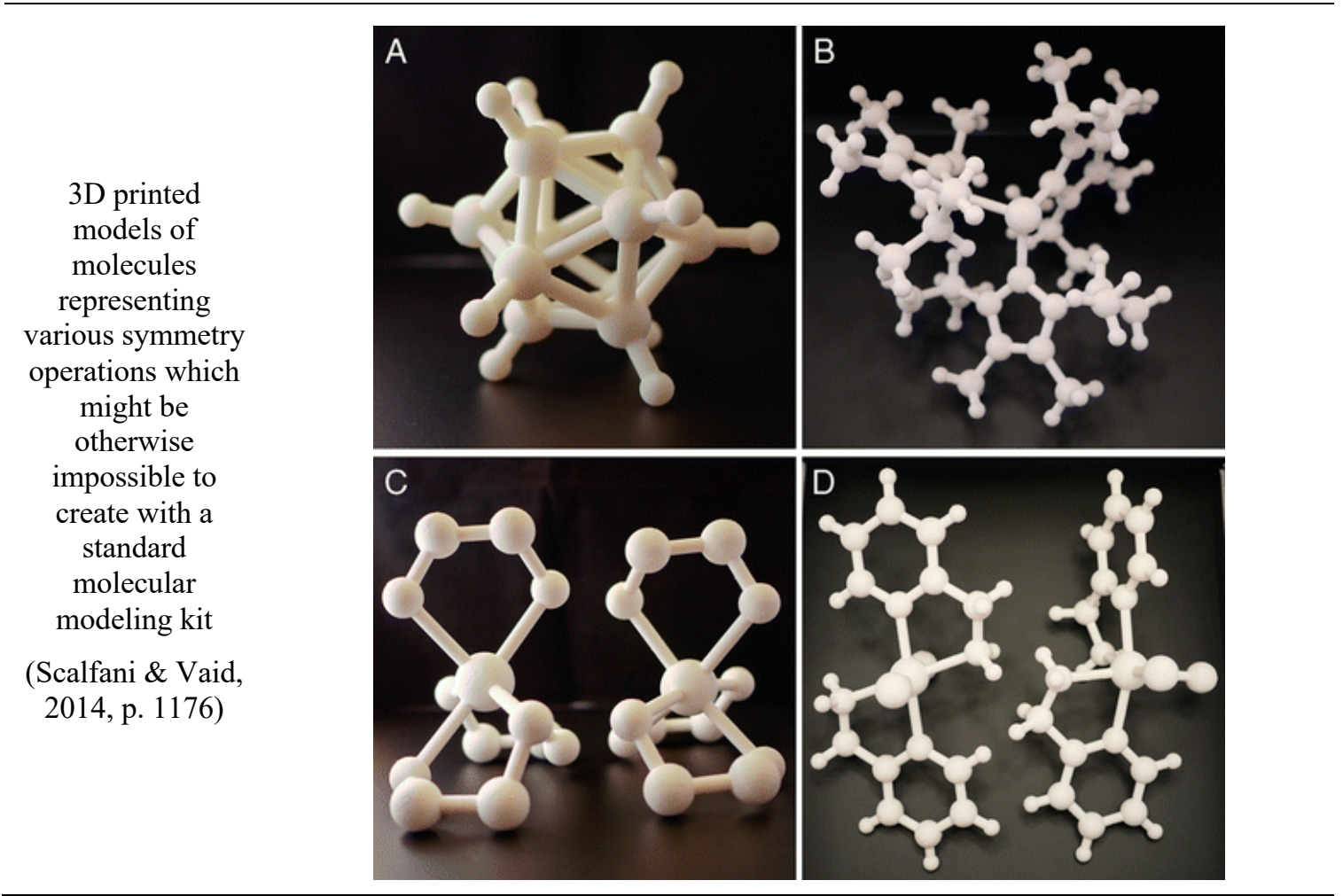

Figure 6. Selected models and manipulatives found in chemistry education literature.

Grafton (2011) presents an alternative way to introduce students to the skills necessary to find and describe symmetry operations in complex three-dimensional objects using high-symmetry dice in a physical chemistry course, as seen in Figure 6 above.

Grafton praises the uniform frame of reference for both students and instructors provided by the index numbers on the sides or vertices. He also describes various ways of painting dice to reduce symmetries such as changing a 4-sided die with a symmetry group of $\mathbf{S}_{\mathbf{4}}$ ( $T_{d}$ in chemical notation) to $\mathbb{Z}_{2} \times \mathbb{Z}_{2}\left(C_{2 v}\right.$ in chemical notation) by coloring one vertex. More recently with the use of 3-d printed models students can now investigate tangible example of molecules that might otherwise be difficult or impossible to assemble from a standard molecular modeling kit which contain symmetry elements that are more easily discerned in a three-dimensional model compared to a two-dimensional illustration. Scalfani and Vaid (2014) provide downloadable files for a collection of 3D printed 
models that are particularly useful for teaching topics such as symmetry and point groups, some of which can be seen in Figure 6 above.

An important and widely used pedagogical resource specifically geared towards inorganic chemistry is the Virtual Inorganic Pedagogical Electronic Resource (VIPEr) which is curated by the Interactive Online Network of Inorganic Chemists (IONiC) (Reisner et al., 2010). VIPEr was founded as a virtual space where specialists in all subfields of inorganic chemistry could come together to share teaching materials and discuss pedagogical approaches to teaching inorganic chemistry content. The website was designed by inorganic chemistry faculty for inorganic chemistry faculty and includes a repository of learning objective such as small instructional units for in-class activities, exam problems, or labs on various topics. There are also social networking tools such as forum and comment threads for help from a rich virtual community of practice among inorganic faculty worldwide (Benatan et al., 2009). Registration and access to all materials are freely available and within the first year of launching the website saw participation from over 250 inorganic chemists from around the world (Benatan et al., 2009). A recent search of the VIPEr website returned over 320 resources related to "symmetry" and over 1000 related to "group theory" (VIPEr, 2019).

\section{$2.3-$ Conclusion}

I have provided an overview of both the mathematics education and chemistry education literature on undergraduate mathematics student thinking related to symmetries and symmetry groups in particular because these are the bodies of literature that I hope to contribute to with the findings of this study. From the mathematics education literature I 
presented an argument for introducing abstract algebra by investigating symmetries to help support the richness of learning opportunities available in the study of this context. This was followed by a survey of various evidence-based instructional innovations within undergraduate abstract algebra which leverage the study of symmetry groups to help describe the ways in which others have used this powerful context in instructional design. The findings from this study will add to these attempts to this general body of attempts, more specifically the LIT created in the proposed study could also fit nicely at the end of the IOAA curriculum as an application module. Lastly, I presented various instructional innovations and student supports for learning symmetry identification and point group classification from the chemistry education community because the LIT created in this study could also be a novel addition to this body of work. 
Chapter 3: Theoretical Perspectives

3.1 - Realistic Mathematics Education

I chose Realistic Mathematics Education (RME) to serve as the underlying instructional design theory for this dissertation experiment. RME is a domain-specific instructional theory for mathematics founded in 1968 in the Netherlands (Van den Heuvel-Panhuizen \& Drijvers, 2014). The theory of RME provides both a theoretical perspective and a theoretical framework. The theoretical perspective of RME is that mathematics is a human activity, and therefore students should be provided with an opportunity to actively participate in the development of mathematical concepts, tools, and procedures. The theoretical framework of RME is outlined in three instructional design heuristics; guided reinvention, didactical phenomenology, and emergent models. These heuristics are used to support students in bridging the gap between their informal mathematical knowledge and more formal mathematics.

\subsection{1 - Theoretical Perspective: Mathematics as a Human Activity}

According to Thompson (1984) any philosophy of mathematics education can be divided into three parts: beliefs about mathematics, beliefs about teaching and learning, and beliefs about mathematics education itself. Thompson goes on to argue that these beliefs are not independent, especially in curriculum development which involves a combination of ideas, theories and notions. Realistic Mathematics Education (RME) is built on the belief and theoretical perspective that mathematics is first and foremost a process, a human activity (Dubinsky, Dautermann, Leron, \& Zazkis, 1994). Gravemeijer (1994) claims that, "when developing a set of instructional activities that makes sense, the developer of RME is guided by beliefs about what mathematics is, how it is learned, 
and how it should be taught. This belief system of the developer functions as the background theory by which all instructional activities are evaluated” (p.111).

For this project I am fully adopting the belief that mathematics is a human activity and am using this belief as the driving force and underlying philosophy. Freudenthal (1971) describes mathematics in the following as a kind of organizing activity:

It is an activity of solving problems, of looking for problems, but it is also an activity of organizing a subject matter. This can be matter from reality which has to be organized according to mathematics patterns if problems from reality have to be solved. It can be mathematical matter, new or old results, of your own or others, which have to be organized according to new ideas, to be better understood, in a broader context, or by and axiomatic approach. (p. 413).

When students are provided with an opportunity to participate in the activity of mathematizing, or the "process by which reality is trimmed to the mathematician's needs and preferences" (Freudenthal, 1973, p.30), the mathematics that they develop is experienced as developing common sense (Gravemeijer, 1998). So, in terms of RME curriculum developed, the emphasis is placed on the nature of the learning process; and on the teaching side, students should be given the opportunity to build on their own mathematical knowledge on the basis of such a learning process. In other words, when basing an instructional design project on the belief that mathematics is a human activity, the learning of mathematics is seen as an active process and the teaching of mathematics as a process of (guided) reinvention. Therefore, as the instructional designer, my aim shifts from just the traditional concrete objectives of sequential tasks, to the educational process itself. 


\subsection{2 - Theoretical Framework: The Three Design Heuristics of RME}

\subsubsection{1 - Guided Reinvention}

The theoretical framework of RME has three accompanying design heuristics, which can serve as both guiding principles for instructional design and as a guide for data analysis both throughout the experiment and retrospectively. These heuristics include the reinvention principle, emergent models, and didactic phenomenology (Gravemeijer, 1998). The reinvention principle suggests that students should be given the opportunities to reinvent mathematics and therefore the context students work in play a key role in the mathematics they develop. Carefully chosen context problems offer opportunities for students to begin by using their own intuitions and experiences to develop informal highly context-specific solution strategies (Gravemeijer \& Doorman, 1999), which students later leverage in a more formal mathematical reality. Not only should the contexts be experientially real to the student, they should offer students opportunities for progressive mathematizing (Gravemeijer \& Doorman, 1999). Through a process of 'guided reinvention' students reflect upon and continue to mathematize their informal solution strategies, ultimately allowing them to 'reinvent' the mathematics they are intended to learn. The context in which students' work may be historically motivated, or it may begin by having students reflect on their own intuitions and interpretations of some natural phenomena (i.e., the shapes of varying molecular structures).

Although 'realistic' situations as 'real-world' situations are important in RME, 'realistic' has a much broader connotation. It means that students are offered problem situations which they can imagine. This interpretation of realistic finds its roots in the Dutch expression "zich realiseren," meaning "to imagine" and it is this emphasis on 
making something real in the mind that gave RME its name (Van den Heuvel-Panhuizen \& Drijvers, 2014). Therefore, in RME the contextualized problems presented to students can be born of the real word, fantasy, or from the formal world of mathematics, so long as the problems and their contexts are experientially real to the students.

\subsubsection{2 - Didactic Phenomenology}

While students may begin by mathematizing everyday-life subject matter, such as a ball and stick model of a specific molecule, the reinvention process demands that they also have an opportunity to mathematize their own mathematical activity (Gravemeijer \& Doorman, 1999). In guided reinvention these distinctions are referred to as horizontal and vertical mathematization. Gravemeijer and Doorman (1999) describe horizontal mathematization as "the process of describing a context problem in mathematical terms to be able to solve it with mathematical means" (p.117). In contrast, Gravemeijer and Doorman define vertical mathematization as “mathematizing one's own mathematical activity" (p.117). The goal of the instructional designer is to lead the students through a series of context problems where students participate in a series of horizontal and vertical mathematizations that together may result in the reinvention of the mathematics one is aiming for. This reinvention is intentionally meant to give students a sense of ownership of the mathematics they create.

The design heuristic of didactic phenomenology motivates the researcher to provide students with contexts (and/or problems) that can be productively mathematized (and/or solved) using exactly the mathematics they are meant to learn. Didactic phenomenology is focused on the relationship between a mathematical content (e.g., concept, definition, 
etc.) and the "phenomenon" it describes and analyses, or, in short, organizes

(Gravemeijer, 2004). A didactic phenomenological analysis can orient a researcher towards contextual problems that can serve as starting points for a process of progressive mathematization. Freudenthal (1986) compared curriculum using didactic phenomenology to more top-down conventional teaching methods, stating,

What a didactical phenomenology can do is to prepare the converse approach: Starting from those phenomena that beg to be organized and, from the starting point, teaching the learner to manipulate these means of organizing. Didactical phenomenology is to be called in to develop plans to realize such an approach. (p. 32)

Didactic phenomenology is useful in both initially selecting a context that can be organized using the mathematics a student is meant to learn and can also be helpful in the ongoing instructional design. Didactic phenomenology can help the researcher in answering the ongoing concern of, 'what kinds of questions and tasks can help students in their reinvention of a particular mathematics through mathematizing the specific context selected?' In this sense, didactic phenomenology is also useful in ongoing analysis. Retrospectively, didactic phenomenology can be used to look at how the specific contexts and tasks aided the students in their ability to reinvent specific formal mathematics.

\subsubsection{3 - Emergent Models}

As students mathematize the given context the activity they participate in can be described as a kind of mathematical modeling. In the RME approach, the models are not pre-derived from the intended mathematics, instead the models are student generated and initially grounded in the contextual problems that the students are meant to solve. Such 
models consist of student strategies, inscriptions, and symbols that together address the contextualized problems; therefore, the term 'model' should be understood in a holistic sense. These models are built out of a kind of modeling activity in which students are engaged in progressive mathematization. In the process of progressive mathematization students mathematize in two distinct ways to construct new mathematics. First, students participate in horizontal mathematization where the contextual problem at hand is described in mathematical terms in order to solve it with mathematical means. Students can then also mathematize their own mathematical activity to reach a higher level of mathematics through vertical mathematization.

It is important to distinguish the type of modeling the students participate in during this type of study from the more common notion of mathematical modeling. Traditionally mathematical modeling can be described as a "translation activity" where students have to translate the problem situations into mathematical expressions that can then function as models. In the more traditional kind of modeling it is important that students are aware of the distinction between the model and the situation so that they can learn to assess whether the model is more or less adequate given the particular goals of the modeler. Alternatively, a model like those found in an RME approach that are the result of an organizing activity emerge from the process of structuring the problem situation. In this kind of modeling the model and the situation modeled co-evolve and are mutually rooted in the organizing activity (Gravemeijer, 2002). Since this type of model is a result of organizing the situation and structuring it in terms of the mathematical relationships, eventually the distinction between the model and the situation modeled dissolves. 
The design heuristic of emergent models is used to describe both the character and the process of evolution of student's formalization of the mathematics they develop. Within this developmental progression, Gravemeijer (1998) discerns four types of mathematical activity, see Figure 7. He describes these as levels but does not impose a strictly ordered hierarchy as students may fold back to earlier levels. The four levels are as follows:

1. the level of situations, where domain-specific, situational knowledge and strategies are used within the context of the situation

2. a referential level, where models and strategies refer to the situation described in the problem

3. a general level, where a mathematical focus on strategies dominates over the reference to the context

4. the level of formal mathematics, where one works with conventional procedures and notions

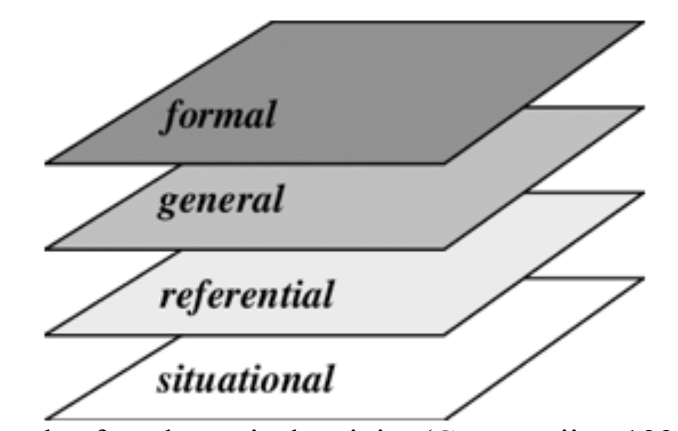

Figure 7. Levels of mathematical activity (Gravemeijer, 1998, p.286-287)

RME models are grounded in the way students initially mathematize a problem within a given context. The students model the problem in order to solve it; and in this sense, modeling is seen as a student mathematizing from which a model of their activity emerges. These models can then mediate a shift from informal situated solution procedures to more formal mathematical reasoning through an evolution process involving where their model of transitions to a model for an unknown concept or context (Gravemeijer \& Stephan, 2002). 
For the student the model is emergent in the sense that the meaning associated with their model shifts over time as they reason with it and continue to mathematize. For the instructional designer the model signifies how students' evolving models work together in reinventing formal mathematics that were grounded in more informally situated activities. A RME approach using emergent models can offer an alternative to classical models of teaching mathematical objects which are often given to students as knowledge to be acquired from an expert who already comprehends it (Gravemeijer \& Stephan, 2002).

The model of the emergent model heuristic globally refers to the evolving process a student undertakes while constructing formal, abstract mathematical knowledge from an initial informal, context-dependent understanding. Gravemeijer highlights three interrelated mechanisms of emergent models: "Firstly, there is the overarching model, which first emerges as a model of informal activity, and then gradually develops into a model for more formal mathematical reasoning. Secondly, the model-of/model-for transition involves the constitution of some new mathematical reality - which can be called formal in relation to the original starting points of the students. Thirdly, in the concrete elaboration of the instructional, there is not one model, but the model is actually shaped as a series of symbolizations" (Gravemeijer, 2002). This heuristic can also be used to describe the qualities and features of the process, not just the process itself. By observing students as their mathematical activity progresses from contextually situated to more formal in a new mathematical reality (Gravemeijer, 1999), the overarching emergent model can be extracted. This global model takes on various manifestations and therefore again, a much broader definition is needed for the term model. The model is 
not simply the inscriptions the students create, but also all of the meaning surrounding these inscriptions, as well as the students' strategies for creating the inscriptions and of using them. Therefore, the various manifestations of the model are represented by the cascade of inscriptions and symbolizations that act as an integral part of the students' organizing activity as they mathematize.

\subsection{3 - Local Instructional Theories}

A local instructional theory (LIT) describes a generalized roadmap for student reinvention of a particular mathematical concept (Gravemeijer, 1998). This path is generalized sequence of steps, described in terms of student strategies and ways of thinking that have been identified as important milestones in the development of the fundamental ideas of the particular mathematical concept (Larsen \& Lockwood, 2013). The theory is local in the sense that it describes how the specific topic should be taught to fit the guiding principles of RME. However, the LIT differs from just a sequence of instructional tasks in the sense that the LIT also focuses on the rationale in choosing such a sequence of activities. The rationale provides an explanation of how the particular instructional activities comply with the intention to give students the opportunity to reinvent mathematics (Gravemeijer, 1998).

Gravemeijer (1998) describes the following key ingredients of local instructional theories:

- informal knowledge and strategies of the students on which the instruction can be built

- contextual problems that can be used to evoke informal knowledge and strategies

- instructional activities that can foster reflective processes which support curtailment, schematization and abstraction (p.280) 
These 'ingredients' are collected and organized into a generalized sequence of steps while rationale slowly emerges. As the researcher reflects upon the design process and upon the instructional sequences themselves the utility of the activity and accompanying rationale are strengthened. LIT's are continually refined through a set of individual research projects, such as a series of teaching experiments. A teaching experiment methodology allows researchers to experience, firsthand, students' mathematical learning and reasoning through an interview like setting where the researcher plays a duel role of teacher/researcher. Instructional activities are tested along with the microtheories that describe how the instructional activities provoke the mental activities of the students, and how these mental activities contribute to the presumed growth in mathematical ability and understanding (Gravemeijer, 1998). Eventually these microtheories serve as the rationale that the curriculum being developed links up with the informal situated knowledge of the students, and they also describe how the curriculum enables the students to develop more sophisticated, abstract, formal knowledge, all the while complying with the basic principle of intellectual autonomy.

Within the undergraduate mathematics education community there already exists a number of well recognized LITs. Previously described is an instructional innovation in abstract algebra using symmetry groups, the IOAA curriculum, which includes LIT's for the concepts of group, isomorphism, and quotient groups, see Chapter 2.1.2.1 - An Evidence Based Instructional Innovation in Abstract Algebra Leveraging Symmetry Groups for more detail. Building on Larsen's reinvention efforts (Larsen, 2009; Larsen \& Lockwood, 2013; Larsen, 2013), Cook (2012a) has focused on the guided reinvention of various concepts in ring theory including ring, integral domain, and field by 
leveraging the context of equation solving. LIT's have also been created for topics within linear algebra including: linear independence and span; matrices as linear transformation; and change of basis, diagonalization, and eigentheory, which compromise the Inquiry Oriented Linear Algebra (IOLA) materials (Wawro, Zandieh, Rasmussen, \& Andrews-Larson, 2013a).

Some of the earliest LIT's created in undergraduate mathematics were in the domain of differential equations, which now include the following topics: solving ODEs; numerical, analytic and graphical solution methods; solutions and spaces of solutions; linear systems; linearization; qualitative analysis of both ODEs and linear systems of ODEs; and structures of solution spaces. Together these materials compromise a first full semester course in differential equations titled, Inquiry Oriented Differential Equations (IODE) (Rasmussen, Keene, Dunmyre, \& Fortune, 2018). Work has also begun in the area of advanced calculus with focus on topics such as the reinvention of the formal definition of limit (Swinyard, 2011), sequence convergence, completeness of the real numbers, and continuity of real functions (Strand, 2016). The findings from the proposed study will both build off of many of the findings from these efforts, and add to this pre-existing body of work with the addition of new LIT.

\subsection{4 - The Ongoing Development of Realistic Mathematics Education}

Roughly half a century has passed from the inception of the development of RME as a domain specific instructional theory, and yet RME can still be seen as a work in progress. It is never considered a fixed and finished theory of mathematics education nor a unified approach to mathematics education (Van den Heuvel-Panhuizen \& Drijvers, 
2014). Researchers who have utilized RME as an instructional design theory have reflected on their own experiences and findings to further the development of RME theory. For example, these contributions have been related to various aspects of teaching and implementing RME based curriculum (Johnson, Caughman, Fredericks, \& Gibson, 2013; Rasmussen \& Marrongelle, 2006) and also aid in further explicating the very design heuristics of RME (Larsen, 2018). While at this time it is not included in the proposed papers section, one way that this this project may also contribute to the greater mathematics education community is through further refinement of RME theory.

Gravemeijer (1998) described four levels of mathematical activity (details in section 3.1.2.3 above) situational, referential, general, and formal. More recently Cook's (2012b) work on guided reinvention in ring theory has expanded on these four phases by expanding the model-of to model-for transition by inserting three sub-phases $(2012 \mathrm{~b}$, p.151-152):

- The situational anticipating referential phase involves activity still firmly rooted in the original situational setting that lays the groundwork for future referential activity.

- The referential anticipating general phase is characterized by models-of that provide an overview of previous work in preparation for abstract or general activity.

- The general anticipating formal phase includes models-for which promote more efficient or concise use of the mathematics at hand in preparation for formal use.

While Gravemeijer claimed that these levels or phases of activity was a non-linear progression with students often folding back, Cook has begun to show that the progression itself may be more complex. I think that this may be one part of RME theory that my study may be able to contribute to by providing even more evidence and 
hopefully explanation for the complexities of the evolution of student mathematical activity through the model-of to model-for progression. 
Chapter 4: Study Methods and Design

4.1 - Design Research

I chose design research as the overarching methodology to test my local instructional theory because a design experiment can help to produce an initial model of successful innovation (Collective, 2003). Design experiments are a type of instructional design theory that have both a pragmatic bent by "engineering" a particular form of learning, and a theoretical orientation which systematically studies the various forms of learning within the context and the means of supporting them (Cobb, Confrey, DiSessa, Lehrer, \& Schauble, 2003), see Figure 8. Through a series of teaching experiments with pairs of students I aimed to understand a route by which a classification system for point groups can be reinvented (Gravemeijer \& Doorman, 1999). Through a design experiment I was able to inquire more broadly into the nature of student learning of group theory in chemistry, refine my local instructional sequences, and build a model for the guided reinvention of point group classification (Collective, 2003).

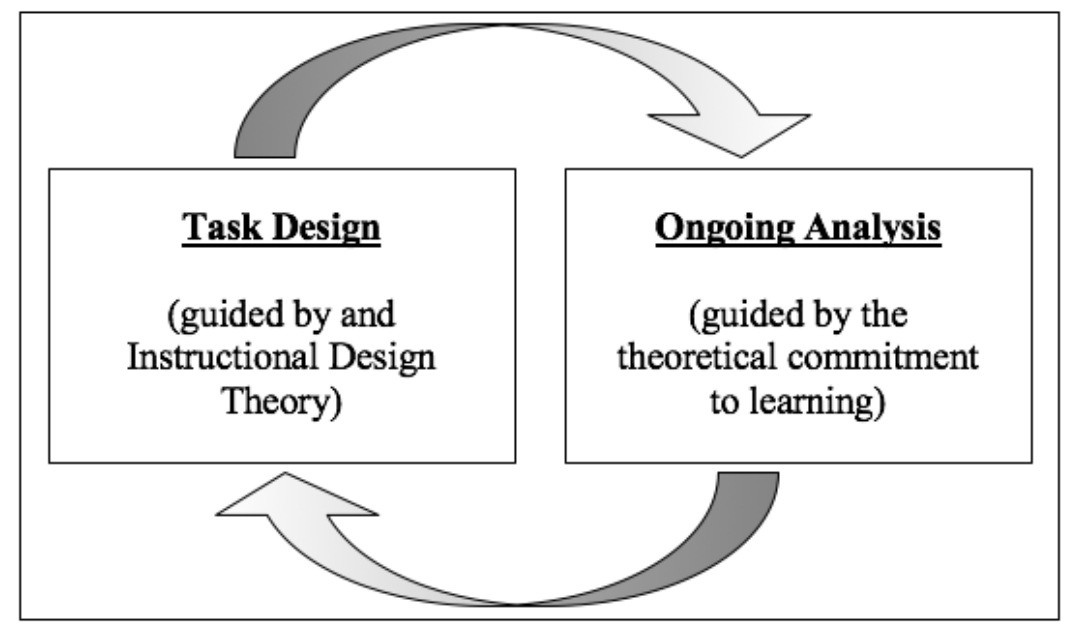

Figure 8. The design research cycle (Wawro, Rasmussen, Zandieh, \& Larson., 2013b)

The experimental method of a design experiment was particularly well suited for testing a preliminary hypothesis such as my preliminary local instructional theory (PLIT) 
since a design experiment has a built-in method refinement. Through three separate stages of research, which I have color coded on my timeline in Figure 9, a design experiment is used to improve the initial design by testing and revisiting conjectures as informed by ongoing analysis of both student reasoning and the learning environment (Cobb, Confrey, DiSessa, Lehrer, \& Schauble, 2003). The first stage is the preparation leading up to the experiment (red), second is conducting the teaching experiments (yellow), and lastly the retrospective analysis (green) (Gravemeijer \& van Eerde, 2009). During the various iterations of teaching experiments, I, the researcher can test my preliminary local instructional theory. While retrospective analysis is the final stage of the design experiment, ongoing analysis is conducted within and between each iteration of the experiment. These complimentary analyses are meant to inform not only the refinement of the protocol for the next experiment, but more importantly the development of the overall local instructional theory (LIT). 


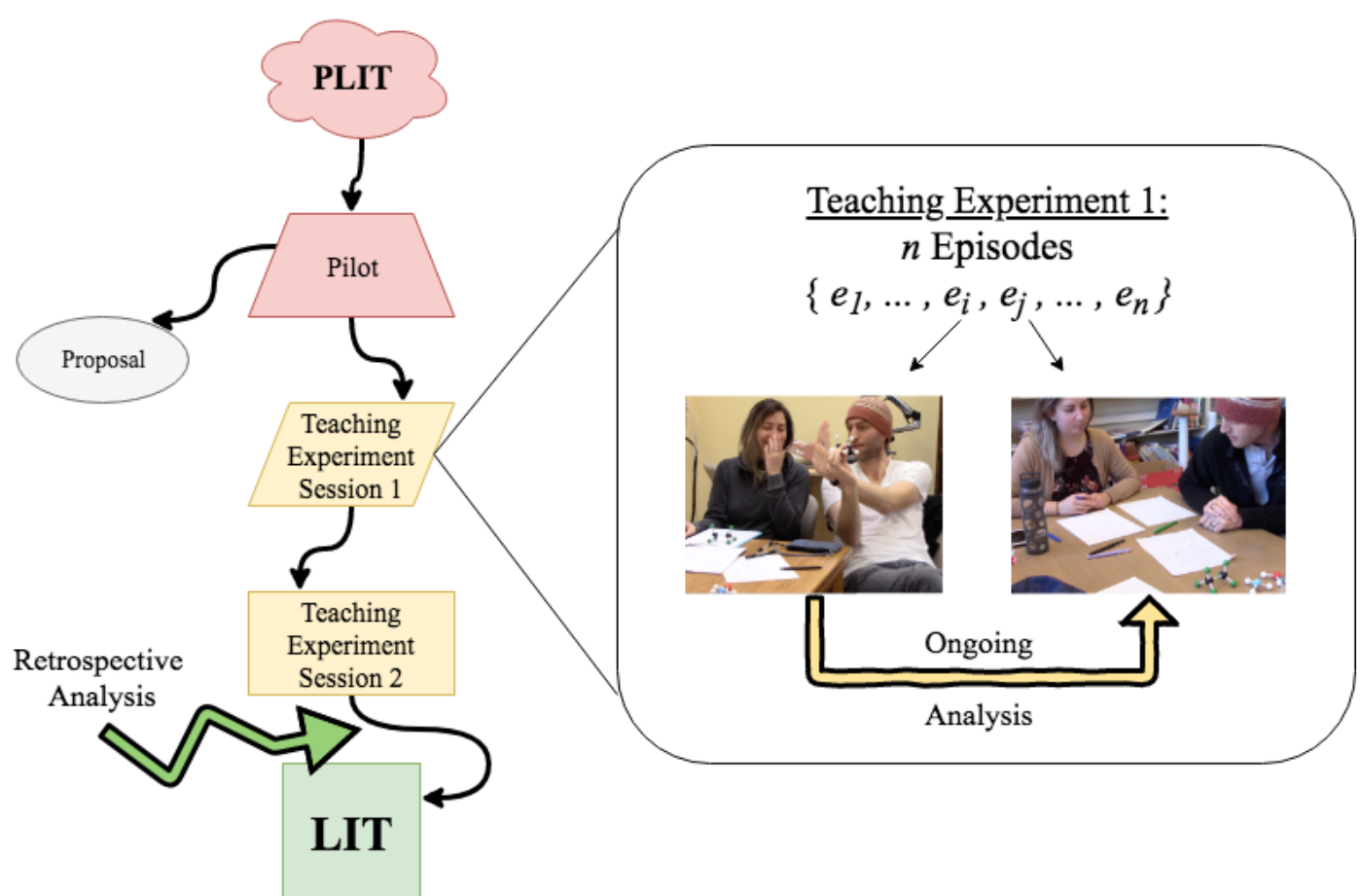

Figure 9. Three stages of design research

\section{2 - Teaching Experiments}

While one classic method for researching student knowledge is the clinical interview, due to the dynamic nature of the learning process, I will argue that a teaching experiment is much more appropriate. Unlike a clinical interview, which is aimed at understanding a student's knowledge at a moment of time, a teaching experiment is directed towards understanding the process of a student's developing knowledge (Steffe \& Thompson, 1991). Larsen and colleagues highlight several reasons for beginning the development of a local instructional theory with a series of teaching experiments with two students instead of a whole classroom setting. As per Larsen et al. (2013);

1. This context provides a chance to work in an idealized setting with no time constraints or curriculum demands to be met. This makes it possible to freely experiment with the tasks design and formulate and test conjectures on the fly.

2. The researcher is able to closely observe each individual student's mathematical activity, which optimizes opportunities to learn from the students how they could be supported in reinventing the mathematics of interest. 
3. Low cost (in terms of time and personnel) and at very little risk. (p.699)

A teaching experiment is a flexible, investigative methodology that can be conducted with individuals, pairs of students, or within a whole classroom setting. I chose to conduct teaching experiments with pairs of students as it allowed me, the teacher/researcher, to focus much more closely on the mathematics of individuals, than a whole class setting might allow. This decision also gave me freedom from any prescribed curricular, pacing, or timing demands from outside stakeholders concerned with the goings on within a classroom setting. Through a series of teaching experiments, I investigated student's mathematical knowledge and how it developed in a teaching context (Steffe, 1991). Also, by working with pairs of participants at a time, I gained greater insight on the development of each individual's mathematical understanding.

The goal of the teaching experiments was to make records of the living models (Steffe and Thompson, 2000) of students' mathematics that could help illustrate aspects of my claims of student reinvention through my local instructional sequence. By conducting multiple teaching experiments, I was able to construct superseding models of student's mathematical understanding, which served in the scientific process of retesting and replication and can helped supply rationale for particular instructional activities. Conducting multiple iterations with pairs of students also helped to provide stability in regard to my claims on student thinking and the appropriateness of my instructional activities. Since the overall goal of the experiment was to develop an instructional approach that promotes both collective and individual mathematical development, it was important to investigate both individual and collective approaches. Cobb \& Bauersfeld (1996) found that, "students' mathematical constructions are both constrained by the 
group's taken as shared basis for communication and contribute to its further development" (p.26).

Teaching experiments not only allowed me to test out my preliminary instructional sequence and a priori hypothesis of student activity, they also allowed me to test hypotheses during the teaching episodes, which were often conceived 'on the fly' (Steffe \& Thompson, 2000). In an instructional sequence designed with the heuristics of realistic mathematics education, a main goal is to have students reinvent the mathematics you want them to learn. This guided reinvention is based first and foremost on the mathematics of the student. Therefore, knowing the students' mathematics is vital in the creation of a local instructional theory as, again it is the student's own mathematical activities that are leveraged to support them through the process of guided reinvention. This project also adheres to the core principle that, mathematics can and should be learned on one's own authority and through one's own mental activity (Gravemeijer, 1998, p. 277). A local instructional theory should include both how the instructional sequence might evoke various mathematical strategies and/or models from the students, and also how these strategies and/or models could be productively used for progressive mathematization (Gravemeijer, 1998). These very resources and rationales can be produced through analysis of teaching experiments (Larsen, 2009).

\section{3 - Data Sources and Subject Selection}

The corpus of data for this study includes a pilot study consisting of a teaching experiment and two follow-up teaching experiments. From here on out, these three teaching experiments will be referred to as TE1, TE2, and TE3, in chronological order. 
The first teaching experiment (TE1) was conducted with a pair of mathematics education graduate students Emmy and Felix who had both completed a yearlong graduate level abstract algebra sequence. This sequence even included a ten-week investigation into the classification of finite groups. The mathematical activity of the students in TE1 severed as a kind of existence proof which informed a preliminary local instructional theory (PLIT) that was then tested and refined in the subsequent experiments. Emmy and Felix were chosen by their willingness to participate and good rapport working with each other and the researchers.

The second teaching experiment (TE2) was conducted with a pair of undergraduate students Arthur and Stu who had recently completed an introductory level group theory course. Arthur and Stu were recommended by their group theory instructor as students who appeared to enjoy introductory group theory concepts and have shown some interest in a deeper understanding in concepts beyond procedural fluency. The students were then contacted via email, offered monetary compensation, and selected by willingness to participate.

For the final teaching experiment (TE3), I once again reached out to a current group theory instructor and unfortunately no students from the list provided by the instructor responded to my request and so alternative students were chosen. Luckily, I had two students in my own 200-level linear algebra course who had shown interest in understanding mathematics more deeply than just procedural fluency and were willing to explicate their thinking. I invited Ada and Sophie to participate in my teaching experiment; by doing so it gave me an opportunity to work with students with a different mathematical background than any of the other students I had previously worked with. 
All sessions were then audio and video recorded; and all written work was collected and scanned into pdfs. A summary of the three teaching experiments is found in Table 1 below.

Table 1. A summary of the three teaching experiments

\begin{tabular}{|l|l|l|}
\hline \multicolumn{1}{|c|}{ TE1 } & \multicolumn{1}{c|}{ TE2 } & \multicolumn{1}{c|}{ TE3 } \\
\hline Emmy \& Felix & Arthur \& Stu & Ada \& Sophie \\
\hline 4 Sessions & 12 Sessions & 11 Sessions \\
\hline $60-80 \mathrm{~min} /$ session & $45-120 \mathrm{~min} / \mathrm{session}$ & $45-90 \mathrm{~min} / \mathrm{session}$ \\
\hline $\begin{array}{l}2 \text { Graduate students in } \\
\text { mathematics education }\end{array}$ & $\begin{array}{l}\text { 2 Undergraduates in } \\
\text { mathematics }\end{array}$ & $\begin{array}{l}1 \text { electrical engineering major } \\
1 \text { mathematics major }\end{array}$ \\
\hline $\begin{array}{l}\text { Ample group theory experience; } \\
\text { including prior graduate level } \\
\text { mathematics experience } \\
\text { classifying finite groups }\end{array}$ & $\begin{array}{l}\text { Both had completed an } \\
\text { introductory group theory course } \\
\text { the previous term }\end{array}$ & $\begin{array}{l}\text { No previous group theory or } \\
\text { proof-based mathematics course }\end{array}$ \\
\hline
\end{tabular}

\section{4 - Ongoing Analysis}

Ongoing analysis was conducted between each episode of each iteration of the teaching experiments. Analysis consisted of re-watching the most recently recorded episode, to identify and describe;

- what progress has been made according to the PLIT

$\circ$ by identifying and describing evidence that the PLIT needs refinement

- analysis of any efforts made in previous ongoing analysis or on the fly to refine the PLIT

- what the goals and direction of the next episode should be

- $\quad$ where to begin the next episode

- what kinds of questions / prompts might be useful in the next episode

\section{5 - Retrospective Analysis}


This study generated over 35 hours of video data, thus making the transcribing of each episode unfeasible. Instead, data analysis began with the creation of content logs for each of the video recorded episodes. A sample content log can be seen in Table 2, and provides an organizational system for analysis notes. Each video was first segmented into 3-minute clips, then each clip was watched while recording a written description of the activity in each. The purpose is to get an overall sense of the sequence of events in the clip, and to start identifying particularly interesting/productive activities for more detailed analysis. More specifically each content log contains:

- An overall description of what happened in the video segment.

- A more detailed description of particularly interesting things that happened.

- Instances where students' thinking moved forward. 
Table 2. Content log excerpt from TE3 Session 10 minutes 12-15.

\begin{tabular}{|c|c|c|c|c|}
\hline $\begin{array}{l}\text { Video } \\
\text { Clip } \\
\end{array}$ & What happened & $\begin{array}{l}\text { RME } \\
\text { Codes }\end{array}$ & $\begin{array}{c}\text { Particularly Interesting } \\
\text { Things } \\
\end{array}$ & $\begin{array}{c}\text { Ways they moved } \\
\text { forward }\end{array}$ \\
\hline TE3_S10_12 to 15 & $\begin{array}{l}\text { [00:00:31.14] I redirect them to Ada's } \\
\text { original flowchart and finally get them to } \\
\text { add the n's to the rotation. Ada does it; } \\
\text { [00:01:16.08] I ask what happens if you } \\
\text { don't have a rotation? I ask them what } \\
\text { happens if you just have a F, T, or F and } \\
\mathrm{T} \text {. } \\
\text { Sophie asks if it would still be one of } \\
\text { their groups? Ada says she thinks it's an } \\
\text { orientation issue. } \\
\text { I ask if you can reorient a reflection into } \\
\text { an orientation, and they both say yes. } \\
\text { Sophie tries to draw something out and } \\
\text { she starts by describing the reflection. }\end{array}$ & & $\begin{array}{l}\text { Ada seems to be struggling } \\
\text { with not having a physical } \\
\text { example and I ask if she } \\
\text { can just imagine just a } \\
\text { reflection and she says that } \\
\text { she would reorient it so } \\
\text { that she was instead } \\
\text { starting with a rotation. }\end{array}$ & $\begin{array}{l}\text { [00:01:16.08] I ask what } \\
\text { happens if you don't have } \\
\text { a rotation? I ask them } \\
\text { what happens if you just } \\
\text { have a F, T, or F and T. }\end{array}$ \\
\hline
\end{tabular}

After I had completed (472 pages) of content logs, I wanted to then look across each of the teaching experiments individually and then across the study as a whole. I started by making documents for each teaching experiment collecting key observations, missed opportunities, etc. that I felt were particularly important from throughout the content logs. Next, in order to better understand similarities and differences between the teaching experiments and across the study as a whole I created a table of all the prompts used in each of the three teaching experiments, found in Appendix B of Paper 3. This table allowed me to first see the consistency of the students' mathematical activity which was abstracted into the LIT described in Chapter 6 : Paper 2. The table also allowed me to distinguish situations where I did things differently from the previous $\mathrm{TE}(\mathrm{s}) ; 1)$ in expectation of differences in students' mathematical backgrounds and/or 2) in response to 
differences in students' mathematical backgrounds which informed much of the findings discussed in Chapter 7 : Paper 3.

For each of the papers presented in the dissertation, various transcripts of video excerpts from the teaching experiments, identified by the content logs and descriptive analysis, are used to highlight the progressive levels of mathematical activity the students participated in. The accompanying symbolizations and inscriptions they constructed through the reinvention process are also provided to show how these observations explicate the overarching emergent model in this work of developing a classification system for chemically important point groups. 
Chapter 5: Paper 1: Developing an Active Approach to Chemistry Based Group Theory

***Notice: this manuscript has already been published elsewhere***

Developing an Active Approach to Chemistry-Based Group Theory Anna Marie Bergman*1 and Timothy A. French ${ }^{2}$

${ }^{1}$ Fariborz Maseeh Department of Mathematics and Statistics, Portland State University

${ }^{2}$ Department of Chemistry and Biochemistry, DePaul University

Group theory, particularly the concept of symmetry, has applications in many different scientific fields and is an important part of the undergraduate curriculum in mathematics. In upper-level mathematics courses, group theory is only discussed abstractly and students are rarely given an opportunity to apply these ideas to real-world problems. In order to better appreciate the applicability of group theory and symmetry, a local instructional theory is being developed where students reinvent a classification scheme for chemically important point groups. In a pilot study, two mathematics education graduate students with limited knowledge of chemistry were given ball-and-stick models of water, ammonia, and ethane and asked to develop and describe a procedure for efficiently and comprehensively finding all the symmetries of any given molecule. Video recordings of the students successfully completing this task and the corresponding inscriptions they made were interpreted using the emergent model heuristic in order to understand the evolution of the students' model from model-of to model-for. Implications of these results on the development of the local instructional theory and for future experiments are also discussed. 
"To be sure, mathematics is a precious treasure-chest of tools, precious that is for those who can put them to good use".

- Hans Freudenthal, mathematician and mathematics educator

Introduction

The use of group theory, in particular the study of symmetry groups has become an essential tool for chemists when interpreting experimental data and predicting the macroscopic properties of materials based on molecular structure. An understanding of molecular symmetry is necessary from a quantum mechanical standpoint as well because molecular wave functions must conform to the symmetry of the equilibrium nuclear framework of the molecule (1). The mathematical consequences of symmetry have profound implications for many important chemical applications, such as: electron configurations, molecular orbital theory, vibrational and rotational motion, optical and NMR spectroscopy. Therefore, as Cotton and Wilkerson explain, 'from a knowledge of symmetry alone it is often possible to reach useful qualitative conclusions about molecular structure and to draw inferences from spectra about molecular structures' (2).

Nowadays, the use of symmetry to describe molecular structure is widely accepted in the chemistry community, so much so that it is commonly introduced and invoked throughout undergraduate courses in organic, inorganic, and physical chemistry. The associated concepts and conventional notation provide evidence towards the creation of a precise description of the underlying structure of symmetry when applied to chemical contexts. For example, the symbol $C_{2 v}$ conveys precise structural information to a trained recipient about a given molecule that could otherwise require long verbal descriptions (2). Because of this utility, the use of symmetry notation in chemistry has become commonplace in the research literature. Therefore, knowledge of the basic concepts, 
conventions, and symbols is necessary in order to read and appreciate the findings reported in many contemporary research papers.

Symmetry is also one of the most powerful and pervasive concepts in mathematics and has intrigued mathematicians for centuries. The mathematical study of symmetry has been systemized and formalized into what is called group theory. Historically, the group concept emerged out of a variety of mathematical lines of inquiry including those in algebra, geometry, number theory, and analysis. Eventually the theory of groups came to be seen as a unifying thread for much of mathematics (3). In general, to a mathematician, a symmetry is an intrinsic property of an object that causes the object to remain invariant under certain classes of transformations. Practically, symmetries offer insight into regularities and are widely applicable in many fields of study, not only in pure and applied mathematics but also in the natural and physical sciences, like the radial symmetry of a sea star or the screw axis of a crystal (4). In general, group theory exploits symmetry when it exists, and the systematic collection of symmetries for a given object can offer powerful insights to the overall structure of the object, like its shape. As Mackey suggests, "many mathematical systems, including those which model the physical world, also have symmetries and symmetry groups and the study of the structural and other properties of these symmetry groups provides profound insights into the more immediately interesting properties of these systems and the key to the solution of many important problems" (5).

Mathematically, a group, $\left\langle G^{*}>\right.$, can be defined as the set of elements $G$ together with an associative binary operation * on $G$. The set $G$ must contain an identity element, contain inverses for each element in the set, and be closed under the operation *. Chemists use a particular set of groups, known as point groups. The set of elements for a point group is a 
set of symmetry operations where each included operation leaves a specific point of a molecule unchanged when applied to a three-dimensional molecular structure. This set of symmetries is then paired with the binary operation of composition of successive symmetry operations (i.e., perform one operation from the set, immediately followed by another-or the same- operation from the set) to form the point group. A symmetry operation applied to a geometrical figure can be thought of as performing some motion to the figure that returns the figure to itself, thus appearing unchanged. For example, a square has a rotational group consisting of only four elements: the symmetry operations corresponding to $90^{\circ}, 180^{\circ}, 270^{\circ}$, and $360^{\circ}$ rotations about the center of a square. The rotational group for a circle, on the other hand, consists of an infinite number of elements because a rotation through any angle about the center of a circle will return the figure back to itself. Although a square and a circle are simple, geometric shapes, this example shows an important implication of group theory when applied to molecular structures-each shape has a different symmetry group that consists of different symmetry operations.

In mathematics, the overall structure of a group is often described in terms of a particular subset of the group called generators. Generators are group elements such that repeated application of these elements on themselves and each other, are capable of producing all the elements in the group (6). For example, the square has a rotational group with four elements, each of which can be produced by composing a $90^{\circ}$ rotation with itself a different number of times. Therefore the rotational group of a square would be described completely as the elements generated by a $90^{\circ}$ rotation. Given the restrictions of three dimensional space and chemical bonding, there are only a relatively small number of combinations of symmetry elements that can occur. Despite the nearly infinite number of 
molecules that can exist, the total number of chemically important symmetry groups is bound to 32 (7). Therefore, group theory is especially powerful for identifying and differentiating molecules based on their valid symmetry operations associated with their shape.

Group Theory in the Undergraduate Curriculum

Due to its powerful ability to simplify problems and guide intuition, symmetry is invoked in undergraduate courses in chemistry. Group theory specifically is often formally introduced in undergraduate inorganic chemistry courses, where the basic formalism of point groups, symmetry operations, and character tables are discussed. These symmetry operations are applied to relatively simple molecules in order to categorize them into their respective point groups. Character tables are also sometimes discussed in physical chemistry courses during units on vibrational spectroscopy. The ACS requires certified graduates in chemistry to have the equivalent of at least one semester of both inorganic and physical chemistry (8). The Committee on Professional Training lists geometries and symmetry point groups as one of the topics in the inorganic chemistry curriculum, but does not require that it be taught (9).

Abstract algebra, the larger field of mathematics containing group theory, is an essential part of the undergraduate mathematics curriculum as well (10-12). According to the most recent CBMS (College Bureau of Mathematics Sciences) report $80 \%$ of departments offering undergraduate degrees in mathematics offered some kind of modern algebra course between 2009-2011, which is an increase in departments offering such courses from $61 \%$ between 2004-2006 (13). Modern algebra is a requirement for a 
bachelor's degree in mathematics in $88 \%$ of mathematics departments. Unfortunately, while many students taking abstract algebra in general, much of the current abstract algebra research highlights student difficulties in learning fundamental concepts in group theory in particular $(14,15)$. As noted in Dubinsky, Dautermann, Leron, and Zazkis, "Mathematics faculty and students generally consider it to be one of the most troublesome undergraduate subjects" (16).

Roughly every ten years the Mathematics Association of America (MAA) charges the Committee on the Undergraduate Program in Mathematics (CUPM) with making recommendations to guide mathematics departments in designing curricula for undergraduates. In 2015, the CUPM shared their most recent recommendations including those specifically for undergraduate curricula in abstract algebra. The CUPM recognizes that, courses in abstract algebra are valuable for a wide variety of students, including mathematics majors and majors in STEM disciplines including chemistry (17). One of the cognitive learning goals highlighted by the CUPM is the integration and application of course concepts, more specifically "students should be able to describe connections between abstract algebra and other mathematics courses they have taken and they should be able to apply algebra to solve problems in other areas of mathematics and in other disciplines" (17). While applications of abstract algebra and connections to other disciplines are being encouraged and promoted by the CUPM, they remain mostly absent in undergraduate group theory courses offered in mathematics departments. A recent study aimed at establishing a meaningful consensus on the valued and important topics of undergraduate group theory amongst experts in the teaching and learning of group theory 
found that applications and/or uses were never mentioned at all, which might be quite shocking to most chemistry faculty (18).

Research focusing on symmetry groups within the chemistry education community also highlights student difficulties with learning symmetry theory in chemical contexts. Students often struggle with visualizing molecules in three dimensions and determining the relevant symmetry operations. "Significant logical-visual spatial skills, including visualization and rotation (i.e. spatial relations), are required to identify symmetry elements and place molecules in point groups. These skills are then partnered with conceptual knowledge to predict vibrational spectra and chirality" (19). However, it has been shown that even with the use of three-dimensional modeling software and the pervasive use of modeling kits, students still have a difficult time visualizing three-dimensional molecular representation of molecular structures (19-21). Students also struggle with the interpretation of complex representations of molecular structures, and then connecting the microscopic structure of a substance to predictions about its macroscopic behavior (20). "Without a robust understanding of the underlying ideas that allow the structure-property connection, there is no organizing framework for most of chemistry and students, out of necessity, resort to memorization, and generation of heuristics" (22).

In traditional instruction, both formal mathematical definitions and rich molecular representations are often presented from the perspective of an expert. These artifacts are representative of complex concepts that are meaningful to the expert given in a relevant representation, which the novice, the student, is meant to extract particular meaning from (23). Renowned mathematician and mathematics educator Hans Freudenthal once said, "to be sure, mathematics is a precious treasure-chest of tools, precious that is for those who 
can put them to good use" (24). Complex and abstract concepts are typically presented in a distilled, ready-made form intended to be easily digestible for students, but in reality these ideas are more often the result of years, decades, or possibly centuries worth of thoughtful pursuits riddled with preliminary conjectures and false attempts. This creates a problem known as "the learning paradox": How is it possible to learn the symbolizations, you need to come to grips with new mathematics, if you have to have mastered this new mathematics to be able to understand those very symbolizations? (25) Too often in traditional instruction the results of the mathematical (and/or chemical) insights of experts taken as the starting point for student activity, rather than from the viewpoint and knowledge base of the student.

\section{Activating Student Learning}

Entering mathematics education from a career as a mathematician Hans Freudenthal saw little use in the ready-made mathematics presented to students and sensed that, "things were upside down if one started by teaching the result of an activity rather than by teaching the activity itself" (26). He characterized this approach to instruction as an anti-didactical inversion. Freudenthal's answer to this inversion was embodied by the idea that mathematics should be taught instead as an activity in which students are expected to participate in what he called mathematizing. Mathematizing can be described as, "the process by which reality is trimmed to the mathematician's need and preferences" (4). Students who are provided an opportunity to reinvent mathematics by mathematizing are given a high level of autonomy and thus the mathematics learned is on one's own and through one's own mental activities. Consequently, this provides students with a sense of 
ownership over the mathematics they reinvent which seems commonsense to the student since it is a product born of their own activities and the mathematics learned has the characteristics of cognitive growth, not of stacking pieces of knowledge (23). Freudenthal argued that this approach to instruction and learning mathematics more honestly represented the activity of actual mathematicians.

University undergraduate classroom instruction can mostly be described as a one-way transfer of information, with homework problems being the primary means of trying to engage students in active learning (27). Within the last half-century various attempts have been made within both chemistry and mathematics education to increase the level of student engagement. Many of the encouraging findings of these studies have recently been amplified in a meta-study conducted by Freeman et al., who analyzed 225 studies that included data on examination scores or failure rates when comparting student performance in undergraduate (STEM) courses (28). Freeman and collaborators found that, on average, student performance on examinations and concept inventories increased by about $6 \%$ in active learning sections, and that students in classes with traditional lecturing were 1.5 times more likely to fail than those in classes with active learning. Freeman's findings raise serious questions about the continued use of traditional lecture methods, and strongly supports active learning as the 'preferred, empirically validated teaching practice in regular classrooms' (28).

A similar meta-analytic study recently analyzed quantitative studies that examine the effects of cooperative learning, CL, on achievement outcomes in chemistry (29). Cooperative learning is defined as, "structured small group activities with five essential components: positive interdependence, face-to-face promotive interactions, individual 
accountability, interpersonal and small group skills, and group processing" (29). In total, twenty five chemical education studies published since 2001 were analyzed, involving 3985 participants. The report found that CL increased student achievement outcomes by 0.68 standard deviations, which implies that a student learning within a CL group setting would perform 25 percentile points better than a student in a traditional group performing at the 50th percentile. The findings of this meta-analysis suggest that cooperative learning is highly recommended as an important pedagogical tool for teaching chemistry at all educational levels.

As the paradigm of STEM education gradually shifts towards cooperative, inquirybased, active learning the call for an increase in student engagement has been answered by educational researchers in both chemistry and mathematics. Process-Oriented Guided Inquiry Learning (POGIL) is one of the most well-known evidence-based curricula in chemistry and is used at both the secondary and post-secondary levels (30). Another pedagogical resource specifically geared towards inorganic chemistry is the Virtual Inorganic Pedagogical Electronic Resource (VIPEr) that is curated by the Interactive Online Network of Inorganic Chemists (IONiC) (31). One important example of researchbased attempts at enhancing student engagement in abstract algebra is the Inquiry-Oriented Abstract Algebra (IOAA) curriculum, which contains materials designed for an introductory group theory course (32). These materials enable students to learn new mathematics through engagement in genuine exploration and argumentation and guide them through the reinvention of important mathematical ideas (33) specifically groups, subgroups, isomorphisms, and quotient groups. 
In the IOAA curriculum students begin by reinventing the group concept via a local instructional theory, best described as a sequence of steps in terms of students' progressive mathematical activity. Guided reinvention of the group concept begins in the context of the symmetries of a equilateral triangle as students identify, describe, and symbolize the set of symmetries. As the students begin to analyze the symmetries of the triangle the group structure begins to emerge as a model-of the students' mathematical activity. As the students' activity transitions from analyzing combinations of symmetries geometrically to calculating combinations algebraically the rules they develop include axioms featured in the definition of group. Students work together to reduce their list to a minimal set of rules needed to completely determine an operation table for combining pairs of symmetries, at which point the students have transitioned from mathematizing the geometric context to mathematizing their own activity. The reinvention process concludes with students analyzing other groups and defining the group concept in terms of the properties shared by these systems (34).

Our Efforts in Activating Student Learning

In an effort to engage students in the richness of group theory and its applicability, the first author (AMB) is currently conducting a design research study aimed to develop a local instructional theory (LIT) for student reinvention of the classification of chemically important symmetry groups. A local instructional theory describes a generalized roadmap for student reinvention of a particular mathematical concept (35). This path is a generalized sequence of steps, described in terms of student strategies and ways of thinking that have been identified as important milestones in the development of the fundamental ideas of a 
particular mathematical concept (36). Both the curriculum and the theory are developed together during the design research process, through a series of teaching experiments, each based on the constructivist teaching experiment $(37,38)$. A teaching experiment methodology allows researchers to experience, firsthand, students' mathematical learning and reasoning through an interview like setting where the researcher plays a duel role of teacher/researcher. Instructional activities are tested along with the microtheories that describe how the instructional activities provoke the mental activities of the students, and how these mental activities contribute to the presumed growth in mathematical ability and understanding (35). Eventually these microtheories serve as the rationale that the curriculum being developed links up with the informal situated knowledge of the students, and they also describe how the curriculum enables the students to develop more sophisticated, abstract, formal knowledge, all the while complying with the basic principle of intellectual autonomy.

As students mathematize the given context the activity they participate in can be described as a kind of mathematical modeling. In the Realistic Mathematics Education (RME) approach, the models are not pre-derived from the intended mathematics, instead the models are student generated and initially grounded in the contextual problems that the students are meant to solve. Such models consist of student strategies, inscriptions, and symbols that together address the contextualized problems; therefore the term 'model' should be understood in a holistic sense. These models are built out of a kind of modeling activity in which students are engaged in progressive mathematization. In the process of progressive mathematization students mathematize in two distinct ways to construct new mathematics. First, students participate in horizontal mathematization where the 
contextual problem at hand is described in mathematical terms in order to solve it with mathematical means. Students can then also mathematize their own mathematical activity to reach a higher level of mathematics through vertical mathematization.

It is important to distinguish the type of modeling the students participate in during this type of study from the more common notion of mathematical modeling. Traditionally mathematical modeling can be described as a "translation activity" where students have to translate the problem situations into mathematical expressions that can then function as models. In the more traditional kind of modeling it is important that students are aware of the distinction between the model and the situation so that they can learn to assess whether the model is more or less adequate given the particular goals of the modeler. Alternatively, a model like those found in an RME approach that are the result of an organizing activity emerge from the process of structuring the problem situation. In this kind of modeling the model and the situation modeled co-evolve and are mutually rooted in the organizing activity (39). Since this type of model is a result of organizing the situation and structuring it in terms of the mathematical relationships, eventually the distinction between the model and the situation modeled dissolves.

\section{Methods}

The overarching goal of a design experiment is to develop a preliminary LIT through a series of teaching experiments with students in order to produce an initial model of successful innovation $(33,36)$. The experimental method of a design experiment is particularly well suited for developing, testing, and refining a preliminary hypothesis, such as a preliminary local instructional theory, since a design experiment has a built-in method 
of refinement through the implementation of multiple iterations. The design experiment methodology distinguishes three stages of research; first stage is the preparation, the second is conducting a series of teaching experiments, and lastly retrospective analysis (41). Additionally, ongoing analysis is conducted within and between each iteration of the experiment. These complimentary ongoing and retrospective analyses are meant to inform not only the refinement of the protocol for the next experiment, but more importantly the development of the overall local instructional theory (LIT).

While the ultimate goal of this project is to develop an LIT that can be used in an undergraduate course, the pilot study was conducted with a pair of graduate students. As per the reinvention principal of RME, before the design research cycle can begin a learning route has to be mapped out along which the students can reinvent the mathematical content for themselves. To do so, the curriculum developer often starts with a thought experiment imaging how they themselves may have reinvented the concept (42). During my thought experiment I imagined that it might not be obvious to students that a classification algorithm for molecular structures was a result of group theory and so I was particularly interested in knowing the extent to which students would use group theory to solve the problem. Therefore, I needed to start with students who knew group theory. Furthermore, the pilot study reported here is only the first part in a larger design research study with multiple teaching experiments planned with pairs of undergraduates.

The mathematical activity and guided reinvention of the graduate students was meant to serve as both a sort of existence proof, and an initial model of success. Graduate students were used in the pilot study instead of undergraduates to better ensure that the students would be able to successfully complete the task of classifying chemically important point 
groups, especially in the amount of time available (4 meetings). As per the design research methodology, the approach of pilot students becomes a hypothetical roadmap for the instructional sequences which will be tested with undergraduates in subsequent iterations of teaching experiments. In this report I will be discussing the findings of the pilot study by sharing the activity of the graduate students, which serves to describe $a$ way in which students might successfully classify chemically important symmetry groups. These findings also raise important questions about the probable / possible approaches of undergraduate mathematics students, which will also be discussed.

The pilot study was conducted with a pair of mathematics education graduate students at a large public university on the west coast, under the approval of an Internal Review Board (IRB). The students, referred to by pseudonyms Emmy and Felix, had both completed a yearlong graduate sequence in abstract algebra including a term in which they classified various groups of finite order. In addition to strong group theory backgrounds the students had good rapport. Emmy and Felix had worked as partners in a previous mathematics course and were extremely supportive work partners, especially in difficult situations. The pilot consisted of four 60 to 90 minute episodes, with time between each episode for ongoing analysis and subsequent construction of appropriate instructional activities based on the ongoing analysis. Data consisted of video recordings of each episode along with all accompanying written work produced by the students. The participants were compensated monetarily for their time.

In order to ensure student engagement and ownership of the knowledge created, the entire study utilizes instructional design theory of RME. The underlying theoretical perspective of RME aligns with Freudenthal's belief that mathematics is first and foremost 
a human activity. Accompanying this perspective is a theoretical framework that includes three design heuristics, the reinvention principal, didactical phenomenology, and emergent models (35). The first heuristic informed the development of the tasks used in the teaching experiment in that a context was chosen that offered an opportunity for the students to begin by using their own intuitions and experiences to develop informal highly contextspecific strategies (23). These context-specific strategies can then be used in a more general mathematical reality. Didactical phenomenology concerns the relationship between a mathematical content and the "phenomenon" it describes and analyses, or, in short, organizes (43). In this sense the heuristic helped at a global level to inform a good starting point, the investigation of particular molecular models, which begged to be organized by the very same mathematical activity intended in the reinvention process. Didactical phenomenology was also used at a more local level during the teaching experiment to drive the study by helping to identify ways in which I as the researcher could support the students transform their informal approaches to specific molecules into more powerful arguments about molecules in general (see Larsen, in press).

Much of the retrospective analysis and data from the pilot are framed using the emergent model heuristic. These models refer to the evolving process a student undertakes while constructing formal, abstract mathematical knowledge from an initial informal, context-dependent understanding. Gravemeijer highlights three interrelated mechanisms of emergent models: "Firstly, there is the overarching model, which first emerges as a model of informal activity, and then gradually develops into a model for more formal mathematical reasoning. Secondly, the model-of/model-for transition involves the constitution of some new mathematical reality - which can be called formal in relation to 
the original starting points of the students. Thirdly, in the concrete elaboration of the instructional, there is not one model, but the model is actually shaped as a series of symbolizations" (39). This heuristic can also be used to describe the qualities and features of the process, not just the process itself. By observing students as their mathematical activity progresses from contextually situated to more formal in a new mathematical reality, the overarching emergent model can be extracted (42). Transcripts of video excerpts from the pilot highlighting the progressive levels of mathematical activity the students participated in and the accompanying symbolizations and inscriptions they constructed through the reinvention process are provided to show how these observations explicate the overarching emergent model in this work of developing a classification system for chemically important point groups.

It should be mentioned that this global model took on various manifestations and a much broader definition for the term model. 'Model' here should be understood in a holistic sense. The model is not simply the inscriptions the students create, but also all of the meaning surrounding these inscriptions, as well as the students' strategies for creating the inscriptions and of using them. Therefore, the various manifestations of the model are represented by the cascade of inscriptions and symbolizations that were an integral part of the students' organizing activity as they investigated the symmetry groups of molecules.

Results

Task setting

In a typical undergraduate inorganic chemistry curriculum students are usually introduced to symmetry theory in a traditional way beginning with definitions. Symmetry 
elements are defined to be geometric objects that a molecule may contain such as mirror planes, rotational axes, and inversion centers, whereas symmetry operations are defined to be the reflections, rotations, and inversions preformed on or about these objects. Once students have a sense of the various symmetries found in 3-dimensional space, they are often given a flowchart, similar to that found in Figure 10, to aid in identifying the specific symmetry group for any given molecule. Much attention is then spent developing students' proficiency in symmetry group identification through memorization of the flowchart and its use along with significant practice applying it to various objects, often starting with simple geometric shapes before moving to molecular shapes of increasing complexity. This drill-based approach reflects a long-held belief that these flowcharts are central to understanding molecular symmetry "since a flow chart serves as a mnemonic device, the beginner very quickly acquires a feeling for molecular symmetry classification" (44). 


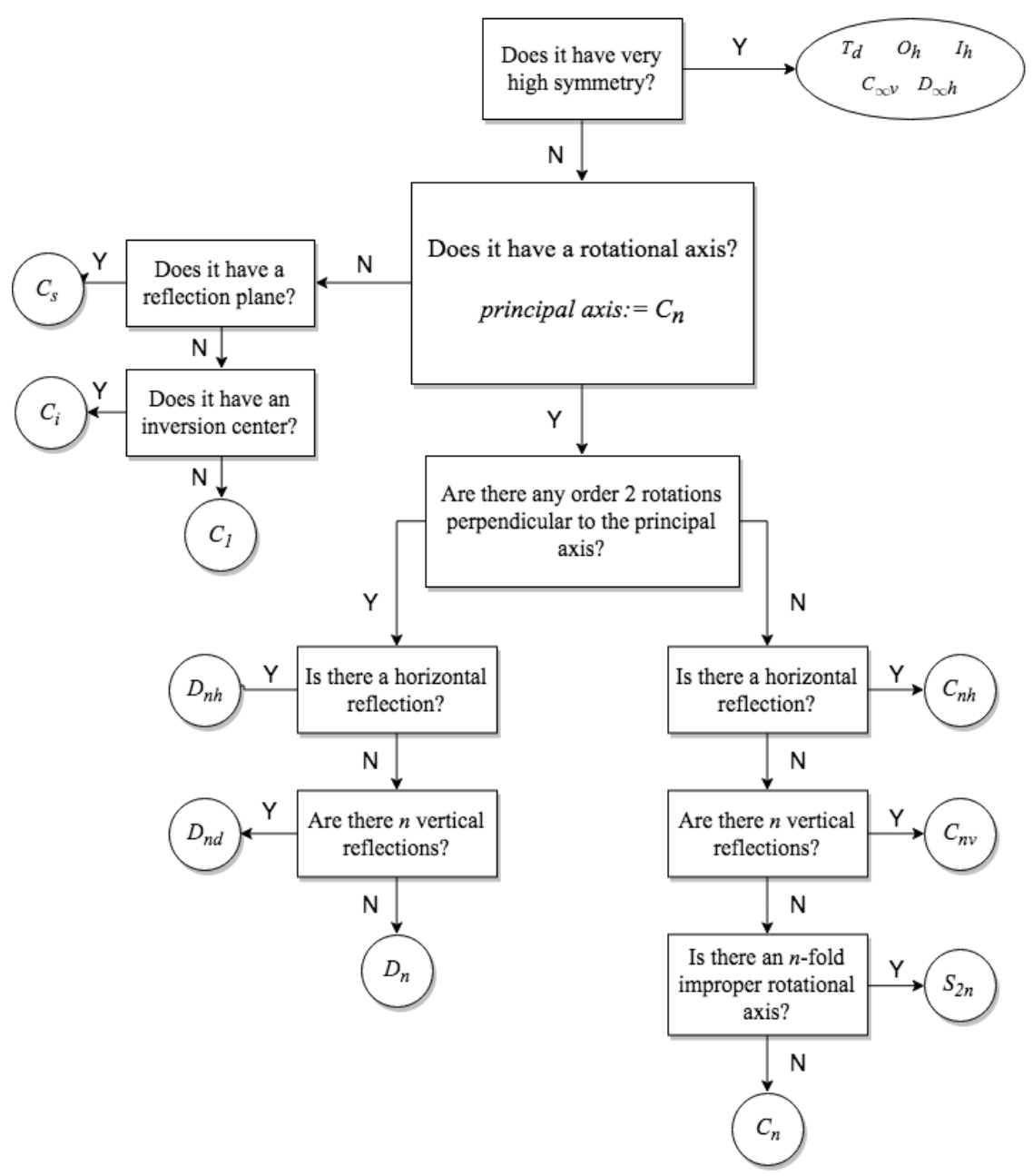

Figure 10. A typical flowchart given to undergraduates in inorganic chemistry to aid in determining the symmetry groups of molecular shapes. Adapted from (45).

An important overall objective of this study is to avoid the antididactical inversion explained earlier, that is, the use of mature, conventional symbolizations as mathematical starting points for instruction. Towards this end, the students had no a priori experience with the conventional classification flowchart; instead, they were given a set of three ball and stick model representations of water, ammonia, and ethane (as seen in Figure 11) and asked "to develop and describe a procedure for efficiently and comprehensively finding all the symmetries of any given molecule." The molecules chosen for the initial task are canonical examples used to introduce symmetry groups, as they contain many, but not all, 
of the symmetry elements present in 3-space. (Notably, they are lacking an inversion center).

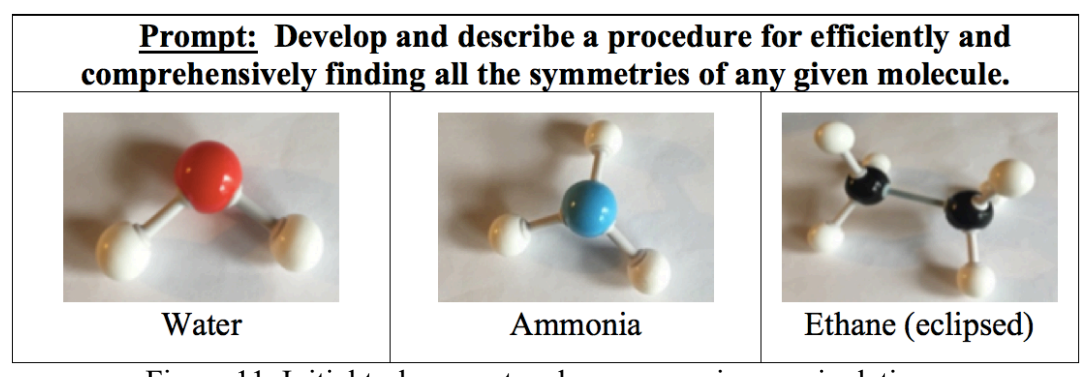

Figure 11. Initial task prompt and accompanying manipulatives.

The resulting activity of the students can be described in two phases:

- Phase 1: activity around finding the symmetry group of a particular molecule

- Phase 2: activity around classifying symmetry groups in general

Phase 1

In this first phase the students' mathematical activity was characterized as situational because their interpretations and solutions were dependent on the symmetry relationships observed within specific molecules and their particular symmetry groups. The pilot students began the experiment by determining and describing the symmetry groups of specific molecules. Both of the students used the same approach 1) identify all symmetry operations to be considered as elements of the symmetry group, 2) distinguish which symmetry operations could and should be considered as generators, 3) determine the relations between each pair of generators, and lastly 4) decide to which 'familiar group' the new found group was isomorphic. Neither of the students wavered from this approach at any time during the pilot, and it proved to be very powerful for them as they were able to successfully identify the unique symmetry group for each molecule. 
While participating in the situated mathematical activity, questions arose that initiated important conversations that were crucial to the students' subsequent success. One of the first realizations the students had was that the structure of a molecule can be considered somewhat fixed due to some underlying set of chemical and physical laws, thus implying that each molecule has a unique shape. Because neither of the student in the pilot had much experience with molecular representations in general, or ball and stick models in particular, they initially wondered whether the atoms could move around the molecule or if the bonds could be broken or if both were possible simultaneously. Almost immediately after grabbing the molecular model of water Felix asks, (while holding the hydrogen atoms), "Can we move these parts? Do these parts not move?" (Ep1, 1:03). Only after Emmy and Felix realized that a unique shape would be necessary to have a well-defined classification system were they able to move forward in the activity of identifying symmetry operations. Determining that the molecules were a fixed shape also allowed them to use their preexisting understanding of triangles while considering the symmetry operations allowed on a water molecule. They quickly determined that the set of symmetry operations of a water molecule would be more similar to that of an isosceles triangle, which is fewer than an equilateral triangle; ultimately this creation of bounds on the problem proved quite fruitful in constructing their procedure.

Another important realization the students made early on during the situational activity occurred when they had to wrestle with the more mathematical question of the definition of a symmetry is in the context of molecular shapes and what these symmetries might look like. Emmy was quick to offer her idea of symmetry (Ep1, 3:24): 
Emmy: So in this case, since these are like 3-d symmetry means if I had a shape, if I had it oriented like this (see Figure 12), I wanna do something (she rotates the model 180 degrees) so that it's in the same orientation?

Felix: I think so yeah.

Emmy: Ok.

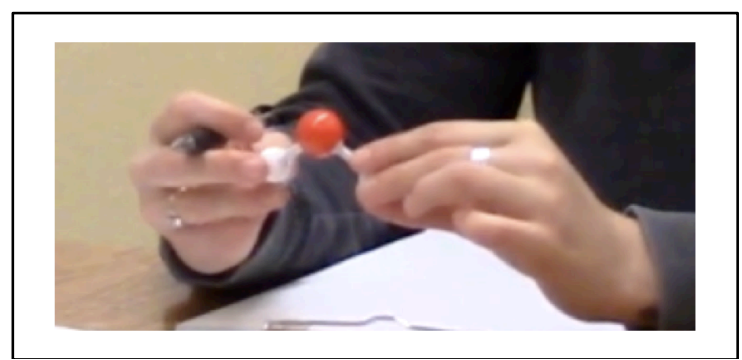

Figure 12. Emmy considers a 180 degree rotation of a water molecule while describing her definition of symmetry.

This description of symmetry aligns closely with the common mathematical definition of a symmetry as "a rigid motion that takes a figure to itself" (32). While the students possessed a productive idea of symmetry in general and extensive experience with both rotations and reflections in two dimensions, the specific rigid motions allowed in the case of molecular structures were less obvious to them. The set of possible "somethings" that Emmy could "do" to the molecule evolved throughout Phase 1. Initially both students seemed to gravitate towards rotational symmetries, presumably because these were tangible motions that could be performed on the models, and subsequently agreed that a water molecule would have at least 2 rotational symmetries, including a trivial 360 degree rotation. When asked if there were any other distance preserving motions other than rotations, Emmy shared that she had an urge to "slice things in half." From this statement, it is reasonable to assume she is considering the existence of mirror planes and whether these are valid symmetries for molecules. Unlike rotations, which can be physically 
performed on the provided molecular models, reflections and inversions must be strictly mental operations due to the rigidity of the ball and stick models; this added an additional concern the students needed to address. Additionally, the result of a 2-dimensional reflection can also be accomplished with a 3-dimensional rotation (out of the plane of the molecule). Though they had extensive experience with 2-dimensional symmetries, 3dimensional symmetries provided a new realm to eventually explore.

Both Emmy and Felix seemed less comfortable with the reflection symmetries, and they continued to refine their idea of reflection throughout Phase 1 by carefully testing their ideas about the effect of a reflection symmetry. Initially their idea of a reflection included the need for the plane of reflection to include an atom from the given molecule. While this constraint worked for both water and ammonia, it failed to describe all the possible reflections observed in ethane. The students wondered about the behavior of specific atoms while considering possible vertical and horizontal reflections. For instance, in the case of ethane in the eclipsed configuration, as seen in Figure 13, Emmy considers a reflection through the plane that is orthogonal to the main (carbon-carbon) bond at its midpoint asking, "are they [the hydrogen atoms] jumping back and forth? Can they do that?" (Ep1, 47:45).

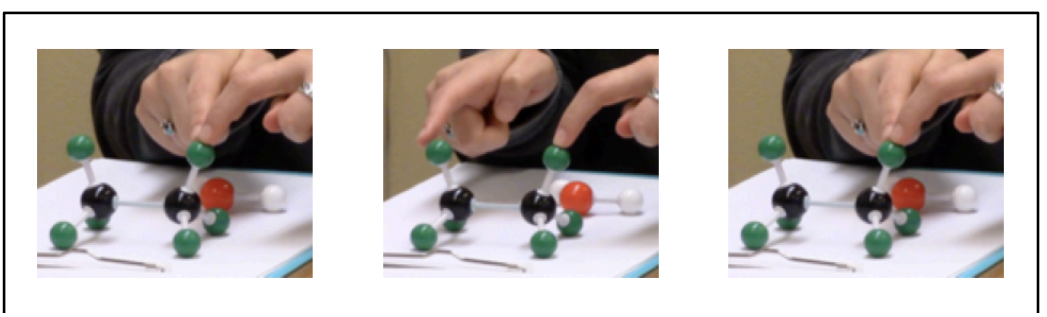

Figure 13. Emmy investigating the effect of "slices", often referred to as horizontal reflections, on ethane. 
Distinguishing vertical planes (those coincident with the principal axis) from horizontal planes (those orthogonal to the principal axis) was important for the students in the situated context, and they spent quite a bit of time discussing how each type of reflection had distinct effects on the peripheral atoms:

Emmy: What we were thinking Annie, was that before when we were slicing stuff, like when we were slicing this guy (grabs the water molecule), we were thinking that the molecules (atom) here (motions to top side of atom, see Figure 14a) are going down to here (motions to bottom side of the same atom, see Figure 14b). But if you slice like that (makes a chopping motion through the center bond of the ethane molecule, see Figure 14c), they don't, that's not what's happening. That's a different kind of slice. (Ep1, 46:23)

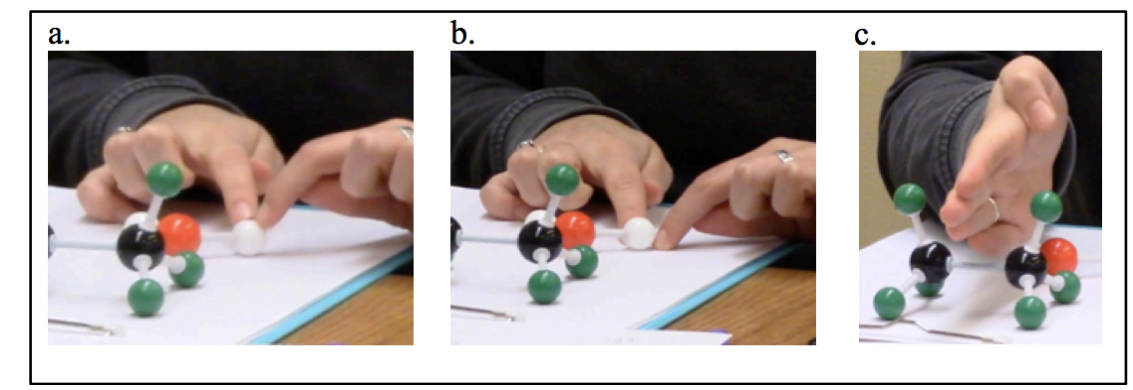

Figure 14. Emmy considers the effects of vertical and horizontal reflections on particular atoms.

Eventually, the pilot students distinguished each of the reflections by giving them different names: reflections through vertical planes they called planes, and reflections through horizontal planes they called slices. The students felt that this differentiation between reflection planes was critical to their progress and it allowed them to accurately describe the symmetry group for each of the given molecules. The distinction between these two types of reflections, coupled with the understanding of how each reflection combines with other symmetry operations, also served as a key mathematical insight into the overall classification algorithm as the various orientations of reflection planes lead to distinct group structures. More specifically, the existence of at least 2 vertical planes yields a group structure with a semi-direct product, which is non-commutative, whereas horizontal 
reflections commute with all other symmetry elements, yielding a group structure containing a direct product.

Lastly, the students determined that certain symmetries, such as inversions or 180 degree rotations orthogonal to the principal axis, could be described using combinations of their previously identified symmetries (principal rotations, reflections coincident with the principal rotations, reflections orthogonal to the principal rotations). For example, when investigating the effect various symmetries had on ethane in both the eclipsed and staggered configurations, the students created a kind of "dot diagram," where the hydrogen atoms were enumerated 1-6 to differentiate them from one another, in order to record and keep track of information about the molecule. The starting orientation (as seen in Figure 15) corresponded to projecting the molecule straight down onto the paper, with the numbers for the atoms pointing towards the observer are drawn closer toward the center. 


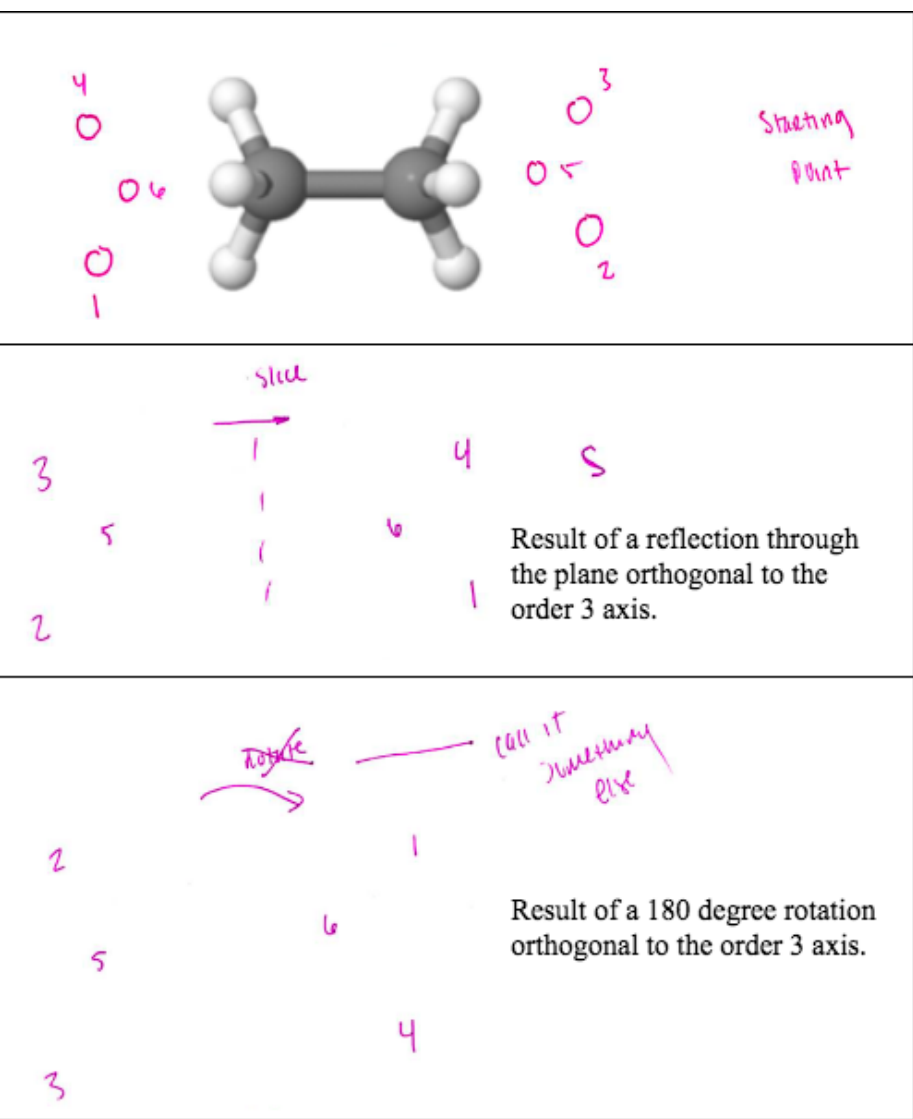

Figure 15. Examples of the students' 'dot diagram' used to determine equivalent symmetries found in the eclipsed configuration of ethane.

As seen in Figure 15, the students used the dot diagram to determine whether a rotation of 180 degrees orthogonal to the principal axis was equivalent to a "slice" (a horizontal reflection). Once they agreed the rotation was not equivalent to simply a slice, they quickly recognized that it was instead equivalent to a slice combined with a "plane" (a vertical reflection). Realizing that this "new" 3-dimensional rotational symmetry was achievable by combining two of their known symmetries reinforced the students belief in their approach of identifying which symmetries could be considered generators. It was this approach that they ultimately reflected on and continued to use throughout their mathematical activity in Phase 2. 
Phase 2

After the students had correctly described the symmetry groups of water, ammonia, and eclipsed ethane, the students' attention was redirected to the original task of classifying symmetry groups in general by redirecting their attention to the original prompt; "develop and describe a procedure for efficiently and comprehensively finding all the symmetries of any given molecule." The students began to reflect on their own experiences with the molecules at hand, signaling a transition of their mathematical activity from situational to a referential activity. During this new mathematical activity their focus shifted to generating models of their own activity in the situational context presented in Phase 1. These models were tested and refined throughout Phase 2 and eventually led to a productive algorithm for identifying a substantial subset of possible symmetry groups. (Due to time restrictions the students were never asked to consider molecules with more than one rotational axis of order greater than two; therefore, they never attended to groups with very high order, such as cubic groups and icosahedral groups.)

The first algorithm they created was a model-of their situational activity and mirrored the reasoning they used to categorize water, ammonia, and eclipsed ethane. Similar to the approach for identifying point groups suggested by the traditional flowchart method, the pilot students started by identifying the highest-order rotational axis, often referred to as the principal axis, of each molecule. After identifying the principal axis, the students attended to both vertical and horizontal reflections. This initial model included both a preliminary flowchart and a kind of "user's manual" that defined the terms they used in their flowchart. It included both their definitions of symmetries and particular assumptions that could not be generalized beyond their observations about the three given molecules 
because this initial model-of originated through reflecting on highly situated activities (see Figure 16). For example, the student's first describe the principal axis as, "rotations about non-hydrogen guys." While this description accurately captures the principal axis in water, ammonia, and ethane, it would fail when considering a molecule with either no hydrogen atoms, such as chlorine pentaflouride $\mathrm{ClF}_{5}$, or a molecule with only hydrogen atoms, such as $\mathrm{H}_{2}$.

Figure 16. Initial model for finding symmetry groups. (Left) Inscriptions associated with the pilot students' initial model-of their approach. (Right) The researcher's (AMB) model-of the students model using more conventional terminology.

Once the students had created an initial model-of their activity in the situated context they began a process of refinement as they continued to reflect on their own activity. The students refined their model in two different ways to accomplish two different goals. First, the students began adjusting their model to better reflect their prior activity with the ball and stick models by testing conjectures about the efficiency of their model. Felix observed, "we need to count rotations for all branches of our flowchart, so perhaps we do that first" 
(Ep3, 3:35). This led them to make a change to their flowchart (Figure 17). The students also refined and expanded their model by considering possible combinations of symmetries that were not present in the three molecular structures provided, and thus had no experience or situational knowledge to build upon. For instance, the two questions Emmy asked"What if there are no planes?" and "What if there are slices and no planes?"- suggests she is thinking about limitations in the current model and possible ways of generalizing it to consider the existence of other molecular shapes. By thinking through these hypothetical (at least, to them) limiting cases, the students further refined their categorization scheme and produced a more robust version of their flowchart (Figure 18). This refinement activity led to an increasingly accurate and usable algorithm for identifying symmetry groups. The lines of questioning centered around the efficiency of the model and the possibility of different molecular structures than those already encountered are examples of "vertical mathematizing", or activities where the reinvented mathematics itself is reorganized, generalized, or formalized (46). 


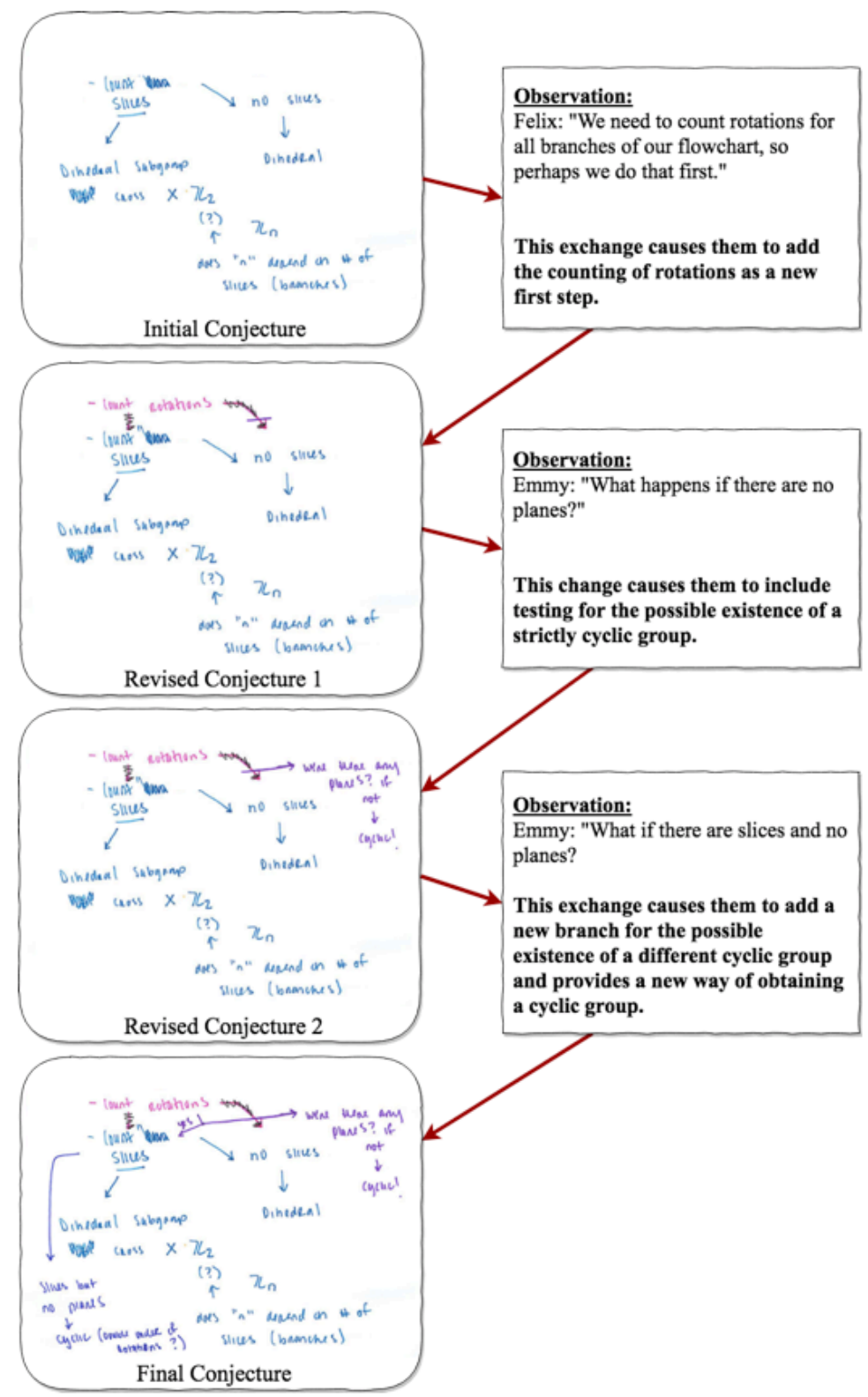

Figure 17. The evolution of the students' initial model through the refining process. The student's observation upon reflection and its corresponding effect on the model are shown for three iterations. Note that the phrase "cyclic group" here refers to a mathematical context, rather than a chemical one. 


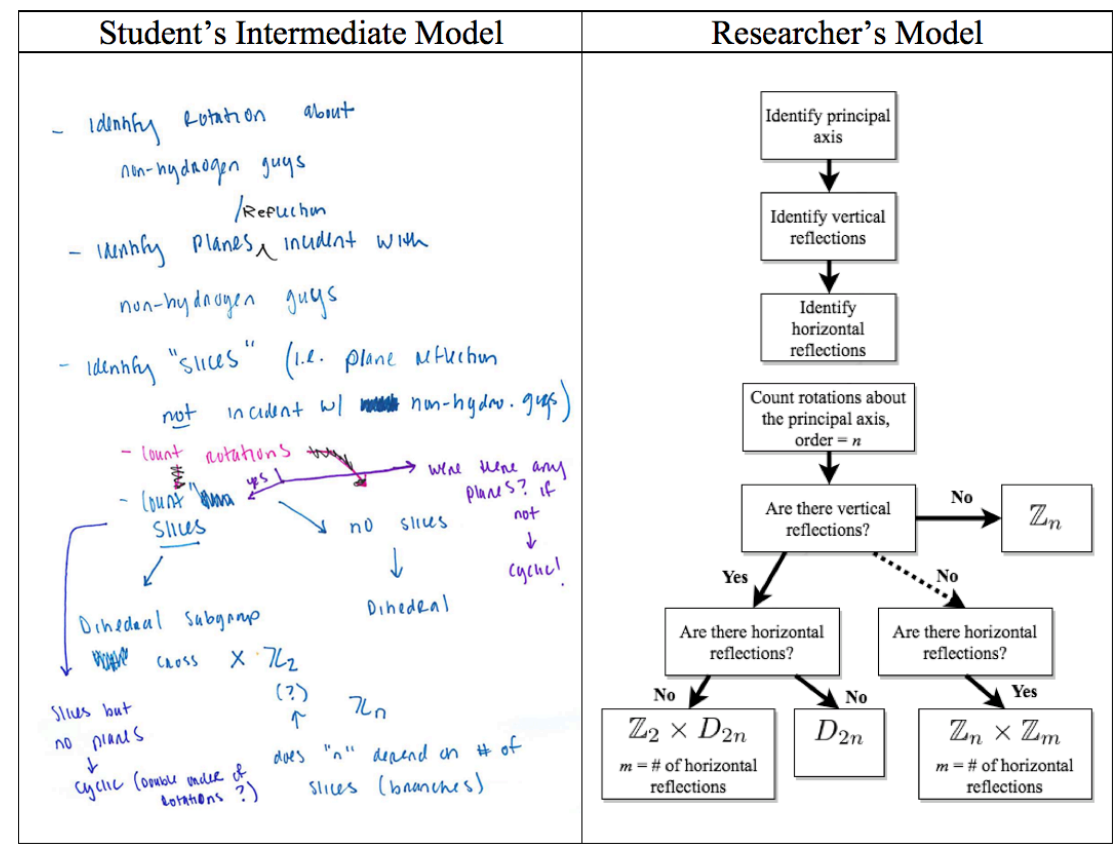

Figure 18. Intermediate model for finding symmetry groups. (Left) Inscriptions associated with the pilot students' intermediate model-of their approach. (Right) The researcher's (AMB) model-of the students model using more conventional terminology.

While constructing their flowchart the students refined many of their descriptions of mathematical objects including which symmetries are valid and how they should be identified. Their initial descriptions were informal and highly contextualized, such as “slicing this guy," “different kind of slice," "identify rotation about non-hydrogen guys." The symmetries at this stage were dependent on the existence and placement of hydrogen atoms in each molecule. Throughout Phase 2, though, the students' descriptions of rotations, planes, and slices became more rigorous through the refining process. The descriptions were less rooted in the context of the initial task and more so on the various geometric entities and symmetry elements found within the molecule under investigation. They also became more formal, general, and mathematically accurate. The evolutions of each of these descriptions can be found in Table 1. 
Table 3. Evolution of the students' descriptions of symmetries. Students' own words are italicized.

\begin{tabular}{|c|c|c|c|c|}
\hline $\begin{array}{c}\text { Students' } \\
\text { Terminology }\end{array}$ & $\begin{array}{c}\text { Accepted } \\
\text { Terminology }\end{array}$ & $\begin{array}{c}\text { Initial Models v.0 - } \\
\text { v.1 }\end{array}$ & $\begin{array}{c}\text { Intermediate Model } \\
\text { v.2 }\end{array}$ & Final Model v.3 \\
\hline Rotation & Principal Axis & $\begin{array}{l}\text { Identify rotation about } \\
\text { non-hydrogen guys }\end{array}$ & $\begin{array}{l}\text { A rotation is a } \\
\text { symmetry about the } \\
\text { axis through the } \\
\text { center of the central } \\
\text { atoms that is } \\
\text { perpendicular to the } \\
\text { plane incident with } \\
\text { non-central atoms }\end{array}$ & $\begin{array}{l}\text { Choose the most } \\
\text { symmetric axis, } \\
\text { i.e. most number } \\
\text { of rotational } \\
\text { symmetries. }\end{array}$ \\
\hline Plane & Vertical Reflection & $\begin{array}{l}\text { Identify } \\
\text { planes/reflection } \\
\text { incident with non- } \\
\text { hydrogen guys }\end{array}$ & $\begin{array}{l}\text { A reflection } \\
\text { symmetry through a } \\
\text { plane incident with } \\
\text { the non-hydrogen } \\
\text { (central atom). }\end{array}$ & $\begin{array}{l}\text { A reflection } \\
\text { symmetry through } \\
\text { a plane incident } \\
\text { with the rotation } \\
\text { axis of symmetry. }\end{array}$ \\
\hline Slice & $\begin{array}{l}\text { Horizontal } \\
\text { Reflection }\end{array}$ & $\begin{array}{l}\text { Identify "slices" i.e. } \\
\text { planes reflections not } \\
\text { incident with non- } \\
\text { hydrogen guys }\end{array}$ & $\begin{array}{l}\text { A reflection } \\
\text { symmetry through a } \\
\text { plane not incident } \\
\text { with the central } \\
\text { atom. }\end{array}$ & $\begin{array}{l}\text { A reflection } \\
\text { symmetry through } \\
\text { a plane } \\
\text { orthogonal to the } \\
\text { rotation axis of } \\
\text { symmetry. }\end{array}$ \\
\hline
\end{tabular}

The students continued to adapt their model via iterations of this “observation-refinement-reflection" process and ultimately created an algorithm for correctly identifying and classifying the symmetry groups of a large subset of possible molecular shapes (Figure 19). The comprehensiveness and scope of the students' reinvented solution compares well to the traditional flowchart provided to chemistry undergraduates (Figure 20). Although the symmetry groups they identified for various molecular shapes were mathematically correct, their level of differentiation between groups was much coarser than that seen in the standard approach found in chemistry. There were four possible distinct groups according to the students' flowchart, whereas the comparable subset of the traditional flowchart shows ten possible distinct groups. The students valued efficiency in their model, and this (along with a background in mathematics rather than chemistry) most likely led to an algorithm that classified chemically important 
symmetry groups by their mathematically isomorphic counterparts, rather than the standard chemical point groups.

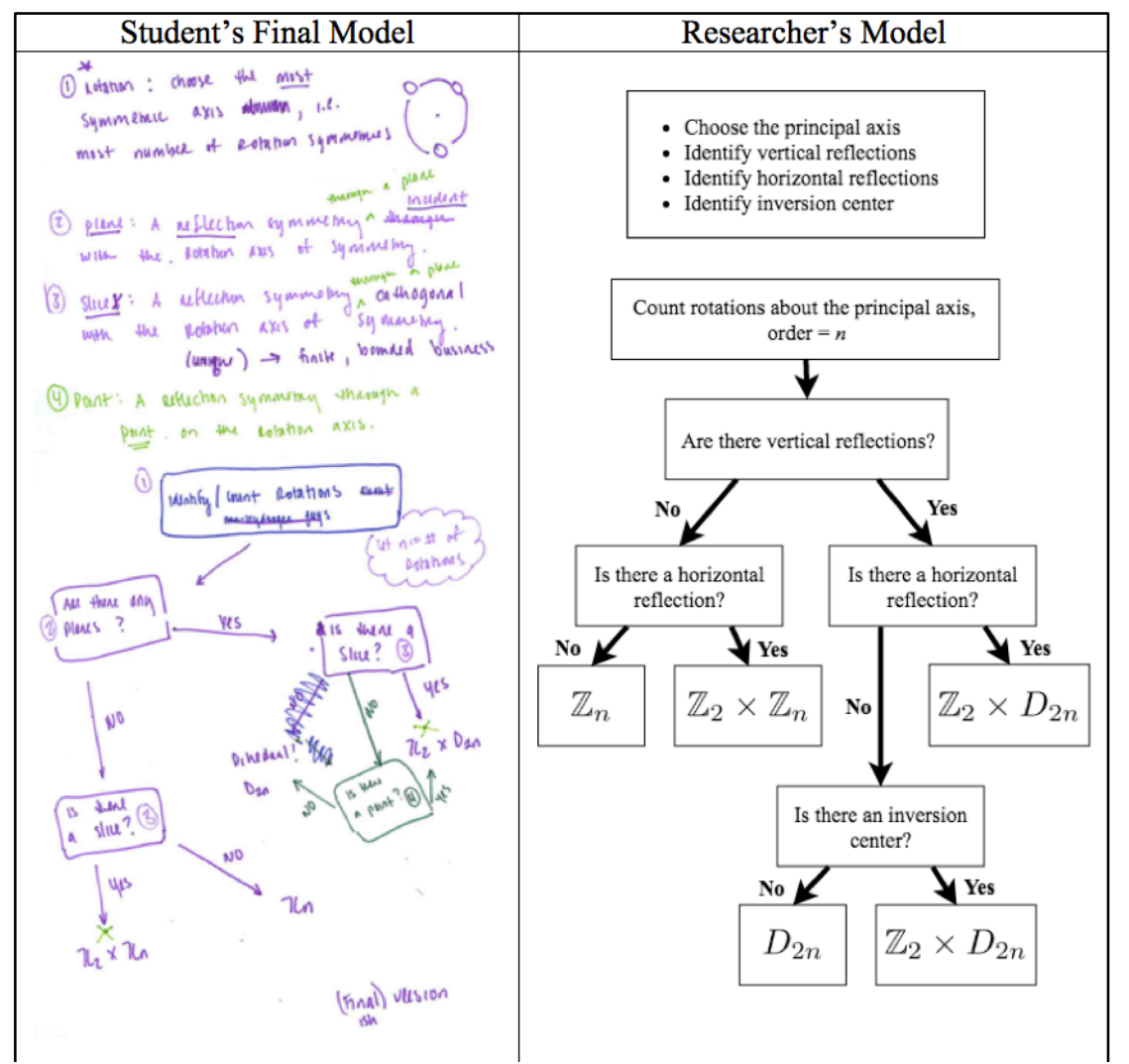

Figure 19. Final model for finding symmetry groups. (Left) Inscriptions associated with the pilot students' intermediate model-of their approach. (Right) The researcher's (AMB) model-of the students model using more conventional terminology. 


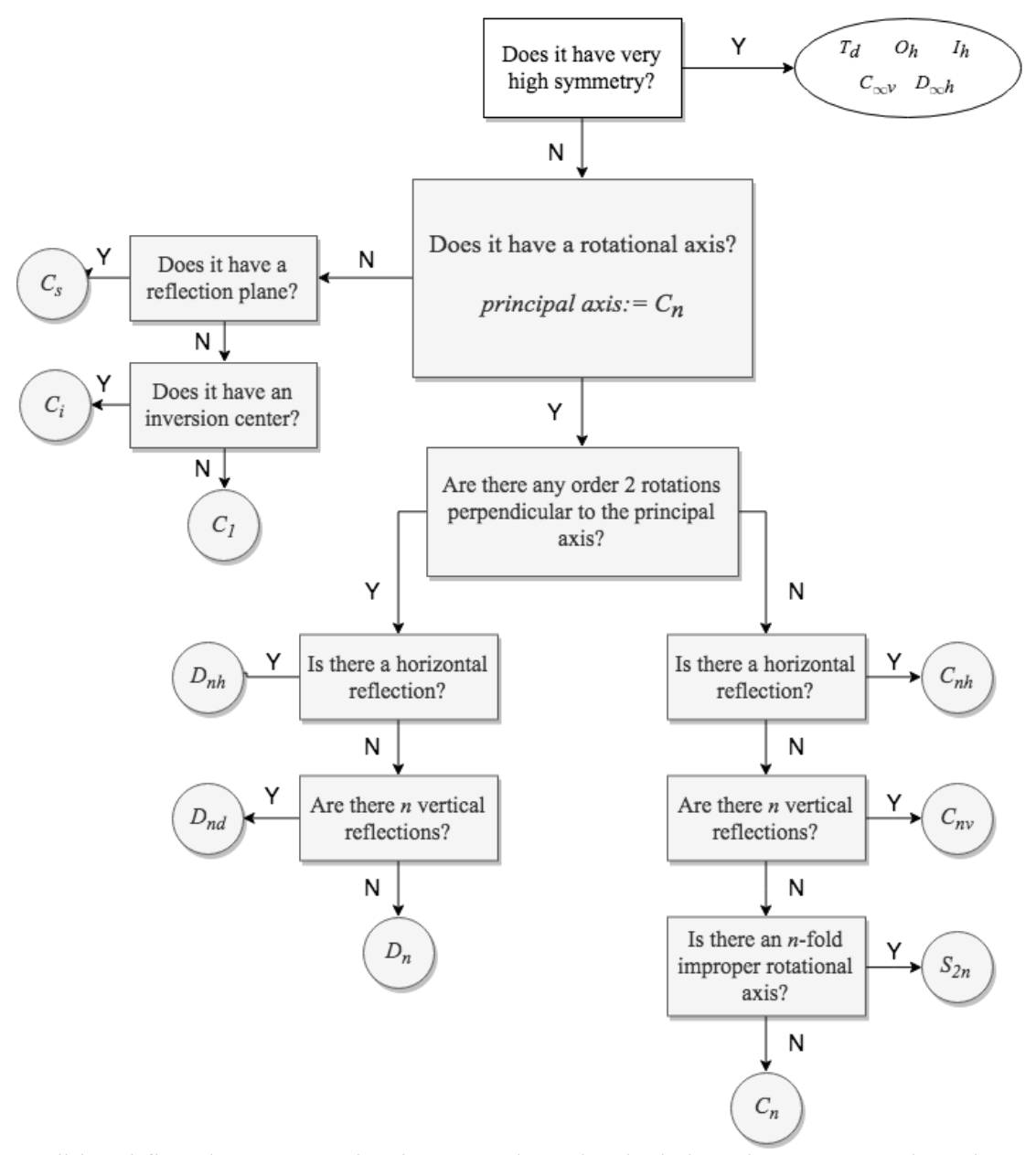

Figure 20. The traditional flowchart, as seen in Figure 1, where the shaded portion corresponds to the symmetry group structures that are identifiable by the algorithm 'reinvented' by the students in the pilot study.

This focus on efficiency was evident when comparing the two flowcharts. The students determined that eclipsed ethane had an equivalent symmetry group as staggered ethane, both of which were isomorphic to the group $D_{2 n} \times \mathbb{Z}_{2}$ according to their final model. Chemists, on the other hand, categorize ethane as $D_{3 h}$ and $D_{3 d}$ for the eclipsed and staggered conformations, respectively. Another difference is that chemists consider the point groups $C_{s}, C_{i}$, and $C_{2}$ to be different from one another even though, mathematically, they are isomorphic to the group $\mathbb{Z}_{2}$ because they have the same structure — an order 2 element and the identity element. Chemists differentiate these isomorphic groups by the identity of the order 2 symmetry element: reflection for $C_{s}$, inversion for $C_{i}$, rotation for $C_{2}$. 
Implications for further study

The disconnect between the mathematically correct and the chemically relevant seen throughout this modeling process presents an interesting source of future work. One important goal of future work would be to learn how to motivate and support mathematics students into developing a scheme that aligns more closely with that of chemists, rather than one that aligns with what algebraists would probably prefer. What is needed is a reason for a chemist to create such a scheme to provide relevance for the chemically important point groups. The discrepancies that arose during the pilot study could provide starting points for this search. For example, the conformations of ethane have different energies in addition to different point groups. Also, the very fact that the order 2 symmetry element in each of these groups is different (reflection for $C_{s}$, inversion for $C_{i}$, rotation for $C_{2}$ ) is a necessary distinction in chemical applications of group theory, such as determining orbital symmetries, calculating reducible representations, and predicting infrared and Raman active vibrational modes. The design heuristic of didactical phenomenology will be particularly useful in discovering a specific problem or context that begs to be organized in a way meaningful to chemists and where the most appropriate solution is the exact organizational scheme the students are meant to learn.

It is important to note that the students during the pilot study were not asked to consider special classes of molecular shapes including linear molecules and groups very high order, such as cubic groups and icosahedral groups. In order to exclude linear groups with infinite rotational subgroups, the pilot students were told that a typical rotational axis in a molecule does not have order higher than 8 , as it would lead to an unstable molecule. The decision 
to limit the task to rotations of finite order helped the students because, from this constraint, they immediately recognized that all the symmetry groups they were meant to discover would be finite. This seemed to reassure the students and offer them a sense of relief that the problem was, in fact, solvable. Groups with very high order were removed from consideration due to time concerns, and students were never asked to consider molecules with more than one rotational axis of order greater than 2 . Because the students never attended to these types of groups, linear molecules and the various classes of highly symmetric molecules will need to be addressed in future work.

Working with graduate students in the pilot study offered both advantages and disadvantages. The approach of the graduate students offers an existence proof of sorts for successfully completing the exercise, which can inform the creation of a preliminary local instructional theory ready to be tested with undergraduates. The pilot were able to create an algorithm to successfully identify the symmetry groups of not only the three molecules they had manipulatives for (water, ammonia, ethane), but also many different hypothetical molecular shapes in a reasonable amount of time. While the mathematical activity of the graduate students offers an approach for creating an algorithm for symmetry group classification, it is important to remember they have had formal instruction on group theory and are entering this task with extensive, structured background knowledge. Their approach was mathematically sophisticated from the start: identifying symmetries, determining which could be considered generating elements, and determining group structure by identifying the relations between various generators. This procedure revealed a sophisticated understanding of group structures and the ways in which they can be constructed. Therefore, the path outlined here may not be one that students with less 
experience with group theory-for whom the LIT and reinvention task is ultimately intended - might find productive or consider at all. In follow-up teaching experiments, it will be important to see how students with a less sophisticated understanding of group theory tackle this classification process. Seeing the variation in multiple student-produced approaches by undergraduates will contribute to the overall robustness of the LIT, as well as provide evidence and rationale for including this LIT as a worthwhile student activity in future courses.

\section{Conclusion}

The overall goal of the work presented here is to create a preliminary local instructional theory which can eventually be developed into a revised LIT for the guided reinvention of an algorithm for the classification of chemically important symmetry groups. This revised LIT will be designed for any undergraduate students, in mathematics or chemistry, who have completed an introductory group theory course. An important aspect of the LIT is its foundation in a real-world application, the identification of symmetry groups for molecules. To determine the tractability of this task and better understand possible student activity during the reinvention process, a pilot study was recently carried out with two mathematics education graduate students who had limited previous knowledge of chemistry and a rich understanding of group theory. These students were given ball-andstick molecular models for water, ammonia, and ethane and ultimately created a classification system for accurately identifying symmetry group structures. Even though they had experience with only these three molecular structures, their algorithm was 
applicable for many other molecular shapes with which they did not have personal familiarity.

The evolution of the students' mathematical activity from informal and contextdependent to more mathematically formal and generalized was observed over time in the video transcripts and corresponding inscriptions produced by the students. Their activity can be broadly described in terms of two distinct phases: classifying specific molecules and generalizing the algorithm for unfamiliar molecules. Interpreting the entirety of the students' activity provides a set of possible productive (and unproductive) lines of inquiry undergraduates may pursue, a better understanding of the construction of knowledge related to group theory, and new research questions to investigate in future, follow-up experiments. The sheer fact that the graduate students successfully accomplished the particular task at hand should not be overlooked, as it shows that a solution is, in fact, possible - an obvious necessity for reinvention that was not known or assumed a priori.

Through further revisions informed by follow up studies with both mathematics and chemistry students this LIT could eventually be developed to serve both populations. This LIT, possibly coupled with the group concept LIT described earlier, could provide an interesting opportunity for chemistry students to gain a deeper understanding of molecular structure (34). Although this specific study immersed mathematics students in a chemical context, this is not a necessary requirement of the method. It is reasonable that chemistry students without formal mathematical knowledge beyond minimal introductory group theory could successfully accomplish this task. It is also reasonable that mathematics students could extrapolate their results and see patterns in chemical properties and behavior given some basic knowledge in chemistry. This LIT could provide chemistry students a 
new theoretical way of considering the implications of molecular shape on chemical and physical problems and mathematics students an opportunity to see how mathematical theories are applied in various fields. Under the heuristic of didactical phenomenology, educators and education researchers can brainstorm new contexts in the subject matter of interest or new concepts relevant to a given context to use in future coursework and experiments. This breadth of applicability is a powerful facet of this method for both instruction and research in mathematics and chemistry.

Knowledge of symmetry theory and its applications is important for practicing chemists and mathematicians to have; therefore, a better understanding of the process by which these concepts are constructed and organized benefits educators and discipline-based educational researchers alike. Typically during the study of symmetry groups, students are introduced to abstract concepts and rich representations that are too often constructed from the perspective of an expert. This manner of presenting material during traditional instruction, where the results and mathematical and chemical insights of others are taken as the starting point for student activity, contributes to what researchers have referred to as "the learning paradox": How is it possible to learn the symbolizations necessary for new mathematics if the very mathematics you are attempting to learn is presented in a manner that assumes its previous mastery? (25) The results discussed here suggest that students can learn the target content by engaging them in the reinvention of the mathematics they are meant to learn. This method leverages the characteristics of cognitive growth, rather than a simple stacking of seemingly independent and possibly unrelated pieces of knowledge, and provides students with a sense of ownership of the knowledge they have created. This work offers a strong basis towards the creation of a pedagogically viable alternative to the 
anti-didactical inversion that students traditionally experience in both undergraduate

mathematics and chemistry courses related to group theory.

\section{References}

1. Cotton, F. A.; Wilkinson, G. Advanced Inorganic Chemistry. New York, NY: Wiley, 1988; Vol. 545, p 28.

2. Kleiner, I. The Evolution of Group Theory: A Brief Survey. Math. Mag. 1986, 59, 195-215.

3. Freudenthal, H. What Groups Mean in Mathematics and What They Should Mean in Mathematical Education. In Developments in Mathematical Education: Proceedings of ICME-2; Howson, A. G., Ed.; Cambridge University Press: Cambridge, UK, 1973; pp 101-114.

4. Mackey, G. W. Group Theory and its Significance for Mathematics and Physics. Proceedings of the American Philosophical Society. 1973, 117, 374-380.

5. Zeldin, M. An Introduction to Molecular Symmetry and Symmetry Point Groups. $J$. Chem. Educ. 1966, 43, 17.

6. Committee on Professional Training. Undergraduate Professional Education in Chemistry: ACS Guidelines and Evaluation Procedures for Bachelor's Degree Programs; American Chemical Sociaty: Washington, DC, 2015.

7. Committee on Professional Training. ACS Guidelines \& Supplements. n.d. https://www.acs.org/content/dam/acsorg/about/governance/committees/training/acsap proved/degreeprogram/inorganic-chemistry-supplement.pdf (accessed Sep 16, 2018).

8. Gallian, J. Contemporary Abstract Algebra. Cengage Learning: Boston, M.A., 2009

9. Hazzan, O. Reducing Abstraction Level When Learning Abstract Algebra Concepts. Educ. Stud. Math., 1999, 40, 71-90.

10. Selden, A.; Selden, J. Errors and Misconceptions in College Level Theorem Proving. In Misconceptions and Educational Strategies in Science and Mathematics, Proceedings of the Second International, Ithaca, New York, July26-29, 1987. Novak, J. D., Ed.; 1987; pp. 457-470.

11. Blair, R. M; Kirkman, E. E; Maxwell, J. W. Statistical Abstract of Undergraduate Programs in the Mathematical Sciences in the United States: Fall 2010 CBMS survey. American Mathematical Society: Washington, D.C., 2013.

12. Larsen, S. Struggling to Disentangle the Associative and Commutative Properties. Learn. Math. 2010, 30, 37-42.

13. Leron, U.; Hazzan, O.; Zazkis, R. Learning Group Isomorphism: A Crossroads of Many Concepts. Educ. Stud. Math. 1995, 29 (2), 153-174.

14. Dubinsky, E.; Dautermann, J.; Leron, U.; Zazkis, R. On learning Fundamental Concepts of Group Theory. Educ. Stud. Math. 1994, 27, 267-305.

15. Melhuish, K. M. The Design and Validation of a Group Theory Concept Inventory. Ph.D Dissertation, Portland State University, Portland, OR, 2015. 
16. Weber, K.; Larsen, S. Teaching and Learning Group Theory. In Making the Connection: Research and Teaching in Undergraduate Mathematics Education; Carlson, M. P., Rasmussen, C., Eds.; Mathematical Association of America: Washington, D.C., 2008, pp. 137-149.

17. Hazzan, O. Reducing abstraction: The case of constructing an operation table for a group. J. Math. Behav. 2001, 20, 163-172.

18. Schumacher, C. S.; Siegel, M. J. CUPM Curriculum Guide to Majors in the Mathematical Sciences. Mathematical Association of America: Washington, DC, 2015.

19. Harle, M; Towns, M. A. Review of Spatial Ability Literature, Its Connection to Chemistry, and Implications for Instruction. J. Chem. Educ. 2010, 88, 351-360.

20. Cooper, M.M.; Underwood, S. M.; Hilley, C. Z.; Klymkowsky, M. W. Development and Assessment of a Molecular Structure and Properties Learning Progression. $J$. Chem. Educ. 2012, 89, 1351-7.

21. Grove, N. P.; Guerin, N. P.; Collins, D. J.; López, J. J; Bretz, S. L.; Zhou, H.- C. Designing, Teaching, and Evaluating a Unit on Symmetry and Crystallography in the High School Classroom. J. Chem. Educ. 2009, 86, 946.

22. Cooper, M. M; Corley, L. M.; Underwood, S. M. An Investigation of College Chemistry Students' Understanding of Structure-Property Relationships. J. Res. Sci. Teach. 2013, 50, 699-721.

23. Gravemeijer, K.; Doorman, M. Context Problems in Realistic Mathematics Education: A Calculus Course as an Example. Educ. Stud. Math. 1999, 39, 111-129.

24. Freudenthal, H. Revisiting Mathematics Education: China Lectures; Kluwer Academic Publishers: Hingham, MA, 1991.

25. Bereiter, C. Toward a Solution of the Learning Paradox. Rev. Educ. Res. 1985, 55, 201-226.

26. Gravemeijer, K.; Terwel, J. Hans Freudenthal: A Mathematician on Didactics and Curriculum Theory. J. Curric. Stud. 2000, 32, 777-796.

27. Black, A. E.; Deci, E.L. The Effects of Instructors' Autonomy Support and Students' Autonomous Motivation on Learning Organic Chemistry: A Self-Determination Theory Perspective. Sci. Educ. 2000, 84, 740-756.

28. Freeman, S.; Eddy, S. L.; McDonough, M.; Smith, M. K., Okoroafor, N.; Jordt, H.; Wenderoth, M. P. Active Learning Increases Student Performance in Science, Engineering, and Mathematics. Proceedings of the National Academy of Sciences. 2014, 111, 8410-8415.

29. Larsen, S. TAAFU: Teaching Abstract Algebra for Understanding. http://www.web.pdx.edu/ slarsen/TAAFU/unit1/lesson1/lesson1.php (accessed Sept 12, 2018).

30. Johnson, E. Inquiry-Oriented Instruction: What It Is and How We Are Trying to Help. http://blogs.ams.org.proxy.lib.pdx.edu/matheducation/2015/04/10/inquiry-orientedinstruction-what-it-is-and-how-we-are-trying-to-help/ (accessed Mar 9, 2017)

31. Moog, R. S.; Spencer, J. N. POGIL: An Overview. In Process Oriented Guided Inquiry Learning (POGIL); ACS Symposium Series; American Chemical Society: 2008; Vol. 994, pp. 1-13. 
32. Reisner, B. A.; Eppley, H. J.; Geselbracht, M. J.; Jamieson, E. R.; Johnson, A. R.; Smith, S.R.; Stewart, J. L; Watson, L. A.; Williams, S. B. Building an online teaching community: an evolving tale of communication, collaboration, and chemistry. In Enhancing Learning with Online Resources, Social Networking, and Digital Libraries. American Chemical Socitey: Washington, D.C., 2010; Vol. 1060, pp. 309330.

33. Gravemeijer, K. Developmental Research as a Research Method. In Mathematics Education as a Research Domain: A Search for Identity. Sierpinksa, A., Kilpatrick, J., Eds.; Kluwer Academic Publishers: Netherlands, 1998, pp. 277-295.

34. Larsen, S.; Lockwood, E. A Local Instructional Theory for the Guided Reinvention of the Quotient Group Concept. J. Math. Behav. 2013, 32, 726-742.

35. Steffe, L. P. The Constructivist Teaching Experiment: Illustrations and Implications. In Radical Constructivism in Mathematics Education. Von Glasersfeld, E., Ed.; Kluwer Academic Publishers: Netherlands, 1991, pp. 177-194.

36. Steffe, L. P; Thompson, P. W. Teaching Experiment Methodology: Underlying Principles and Essential Elements. In Handbook of Research Design in Mathematics and Science Education. Kelly, A. E., Lesh, R. A., Eds.; Routledge: New York, NY, 2000, pp. 267-306.

37. Gravemeijer, K. Preamble: From Models to Modeling. In Symbolizing, Modeling and Tool Use in Mathematics Education. Gravemeijer K., Lehrer R., Van Oers B., Verschaffel L., Eds.; Springer: Dordrecht, 2002. pp. 7-22.

38. Collective, T.D. - B.R. Design-based Research: An Emerging Paradigm for Educational Inquiry. Educ. Res. 2003, 32, 5-8.

39. Gravemeijer, K.; van Eerde, D. Design Research as a Means for Building a Knowledge Base for Teachers and Teaching in Mathematics Education. Elem. Sch. J. 2009, 109, 510-524.

40. Gravemeijer, K. Local Instruction Theories as Means of Support for Teachers in Reform Mathematics Education. Math. Thinking Learn. 2004, 6 (2), 105-128.

41. Gravemeijer, K. How Emergent Models may Foster the Constitution of Formal Mathematics. Math. Thinking Learn. 1999, 1, 155-177.

42. Carter, R. L. A Flow-Chart Approach to Point Group Classification. J. Chem. Educ. 1968, 45, 44.

43. Treffers, A. Three dimensions: A model of goel and theory description in mathematics instruction - The Wiskobas Project; Reidel: Dordrecht, The Netherlands, 1987. 
Chapter 6: Paper 2: A Local Instructional Theory for the Guided Reinvention of a Classification Algorithm for Chemically Important Symmetry Groups

A Local Instructional Theory for the Guided Reinvention of a Classification Algorithm for Chemically Important Symmetry Groups

\section{Anna Marie Bergman}

In this paper I describe a local instructional theory (LIT) for the guided reinvention of a classification system used for identifying symmetry groups of molecules. This LIT is the result of a design study focused on students work with symmetry groups in the context of chemistry. This local instructional theory consists of both a generalized instructional sequence intended to support the guided reinvention of a classification algorithm for molecular structures, and the theoretical and empirical rationale for the given sequence. The key steps in the sequence are described in terms of students' mathematical activity and evidence from the various teaching experiments is used to further explicate important aspects of the reinvention process.

This paper describes a local instructional theory (LIT) for the guided reinvention for a classification system for chemically important symmetry groups. This LIT is the main deliverable from a design study aiming to develop a theory of how students might reinvent a classification system for point groups. The instructional goal of the study was to engage mathematics students in an authentic application of group theory using guided reinvention. The generalized instructional sequence found in the pages to follow is described in term of students' mathematical activity during the reinvention process. Each step in the sequence is illustrated using instructional tasks and evidence of students' mathematics in the form of excerpts and written work collected through a series of teaching experiments. This research fits with the growing body of work using the 
instructional design theory of Realistic Mathematics Education to support the student understanding of various mathematical concepts at the undergraduate level, (Larsen, Johnson, \& Bartlo, 2013; Rasmussen, 2007; Wawro, Rasmussen, Zandieh, \& Larson (2013); Zandieh \& Rasmussen, 2010).

Keywords: Realistic Mathematics Education, Abstract Algebra, Group Theory, Symmetry

\section{Background}

Abstract algebra has long been considered a challenging course (Leron, \& Dubinsky, 1995). Both "mathematics faculty and students generally consider (abstract algebra) to be one of the most troublesome undergraduate subjects" (Dubinsky et al., 1994, p.268). The content is abstract, axiomatic, and complex (Melhuish, 2015), where many of the fundamental concepts such as isomorphism require a complex coordination of many ideas (Leron, Hazzan, \& Zazkis, 1995). Furthermore it is also a course in which students are often learning how to prove, "for most undergraduates this course is one of their earliest experiences in coping with the difficult notions of mathematical abstraction and formal proof" (Weber \& Larsen, 2008, p.139). The small body of existing literature related to student thinking in abstract algebra unanimously documents the difficulty of student's conceptual understandings of various topics (Melhuish, 2015). This has led the majority of research on the teaching and learning of undergraduate abstract algebra to fall into one of two categories: student misconceptions and instructional innovations (Weber \& Larsen, 2008). 
For many, abstract algebra is the first course in which students are asked to engage with concepts that are brought into existence via formal definitions. In other words, concepts are defined and presented often by a list of relevant properties followed by an examination of 'what facts can be determined just from [the properties] alone.' (Dubinsky et al., 1994). Hazzan (1999) found that this new mathematical style of presentation can lead students to adopt mental strategies to enable them to mentally cope with the new approach, as well as with the new kind of mathematical objects. In particular, Hazzan provided a framework for three distinct ways in which students reduce abstraction in order to cope with the abstract nature of the content. More recent work (Melhuish, Bergman, Czocher, 2018) has extended this framework of mental strategies purposed by Hazzan to include eleven different kinds of mental strategies for navigating abstraction that students may employ when learning abstract algebra.

One response to these student struggles from both instructors and instructional designers has been to try and find ways to help students understand abstract algebra concepts in ways that are more obviously relevant for the students, and less initially abstract. Nearly half a century ago legendary mathematician turned mathematics educator Hans Freudenthal (1973) argued for teaching abstract algebra, in particular group theory, by first investigating automorphisms of sets. He posited that "groups are important because they arise from structures as systems of automorphisms of those structures" (1973, p.109), and that investigating these sets of automorphisms is a natural way for students to approach group theory that builds on their intuitions and informal approaches. Larsen (2004) took this suggestion to heart when first designing his own instructional innovation, the evidence-based Inquiry Oriented Abstract Algebra (IOAA) 
curriculum. The termlong IOAA curriculum begins with the investigation of the symmetries of an equilateral triangle as an entry point to the guided reinvention of the group concept before continuing on to support students in reinventing other key concepts such as isomorphisms and quotient groups (Larsen, 2013). Initial investigations into the efficacy of this curriculum support both its ability to evoke desirable mathematical activities such as defining, conjecturing, and proving; and for promoting student understanding of key abstract algebra concepts (Larsen, Johnson, \& Bartlo, 2013).

For this study I aimed to develop a generalized instructional sequence which capitalizes on the potential Freudenthal (1973) described and Larsen (2013) found introducing abstract algebra with an investigation of a system of automorphisms of a structure under composition. Building on their suggestions and success, I would like go one step further by presenting students with symmetries in a real-world context. I chose the context of chemistry because undergraduate inorganic chemistry students often learn group theory as a tool to help differentiate molecular structures (Raker et al., 2015a, 2015b).

The classification of chemically important symmetry groups

In experimental chemistry much of the work involves understanding and interpreting the nature of chemical compounds. Chemists use an understanding of symmetry theory to completely and rigorously answer the question, "What is possible, and what is completely impossible?" Cotton (1988) claimed that by symmetry considerations alone, chemists can always gain insight into the qualitative features of the molecule without any quantitative calculations such as how many energy states there are and also what 
interactions and transitions may occur between them. Symmetry theory is particularly powerful in inorganic chemistry where molecules tend to be smaller and more symmetric than their larger organic counterparts. Even undergraduate chemistry students are taught to use symmetry concepts "to predict infrared spectra, describe orbitals used in bonding, predict optical activity, interpret electronic spectra, and study a number of additional molecular properties" of molecules (Miessler et al., 2014, p.75).

One way in which chemists gain insight on molecular structures is by determining their symmetry group, known to a chemist as its point group. The set of elements for a point group is a set of symmetry operations (transformations which return the molecule to itself while preserving distances) where each included operation leaves a specific point (or set of points) of a molecule unchanged when applied to a three-dimensional molecular structure. This set of symmetries is then paired with the binary operation of composition. There are only a relatively small number of symmetry groups that can occur, limited by both mathematics (possible symmetries in three-dimensional space) and chemistry (too many atoms in a single plane will produce too weak a bond to stay stable). The total number of chemically important symmetry groups is bound to 32 (Zeldin, 1966). In summary, group theory is especially powerful for identifying and differentiating molecules based on the valid symmetry operations associated with their shape. My goal is to engage mathematics students in the classification of these symmetry groups to better understand both shapes of molecular structures and to deepen their understanding of algebraic structures, meanwhile enriching their understanding of abstract algebra. It is important to reiterate that my goal is to engage mathematics students, and therefore their 
approaches to using symmetries to differentiate shapes and structures, will necessarily be different form that of a chemist.

Chemists explicitly distinguish between symmetry elements and symmetry operations Symmetry elements are mirror planes, rotational axes, and inversion centers. In other words, the geometric entities with respect to which one or more symmetry operations may be carried out. Symmetry operations are the movements, such as reflections, rotations, and inversion; the angle and distance preserving motions that return the molecule to its original orientation. In mathematics we too consider symmetry in two different ways, although not the same two. We mathematicians consider symmetry as a property of a figure, and as a rigid motion (isometry) that maps a figure to itself. Chemists identify four kinds of non-trivial symmetry elements and their associated symmetry operations, described in Table 4 below.

Table 4. Summary table for symmetry elements and operations typically used in an inorganic chemistry course

\begin{tabular}{ll}
\hline \multicolumn{1}{c}{ Symmetry element } & \multicolumn{1}{c}{ Symmetry Operation(s) } \\
\hline 1. Plane & Reflection in the plane \\
2. Inversion center & Inversion of all atoms through the center \\
3. Proper rotational axis & One or more rotations about the axis \\
4. Improper rotational axis & $\begin{array}{l}\text { A rotation followed by a reflection through a } \\
\text { plane perpendicular to the axis of rotation }\end{array}$
\end{tabular}

Once a chemist has identified the symmetry operations available to a particular molecule, through the existence of various symmetry elements, they then use an algorithm, Figure 21, to identify the symmetry classification of the molecule in terms of their symmetry group. 


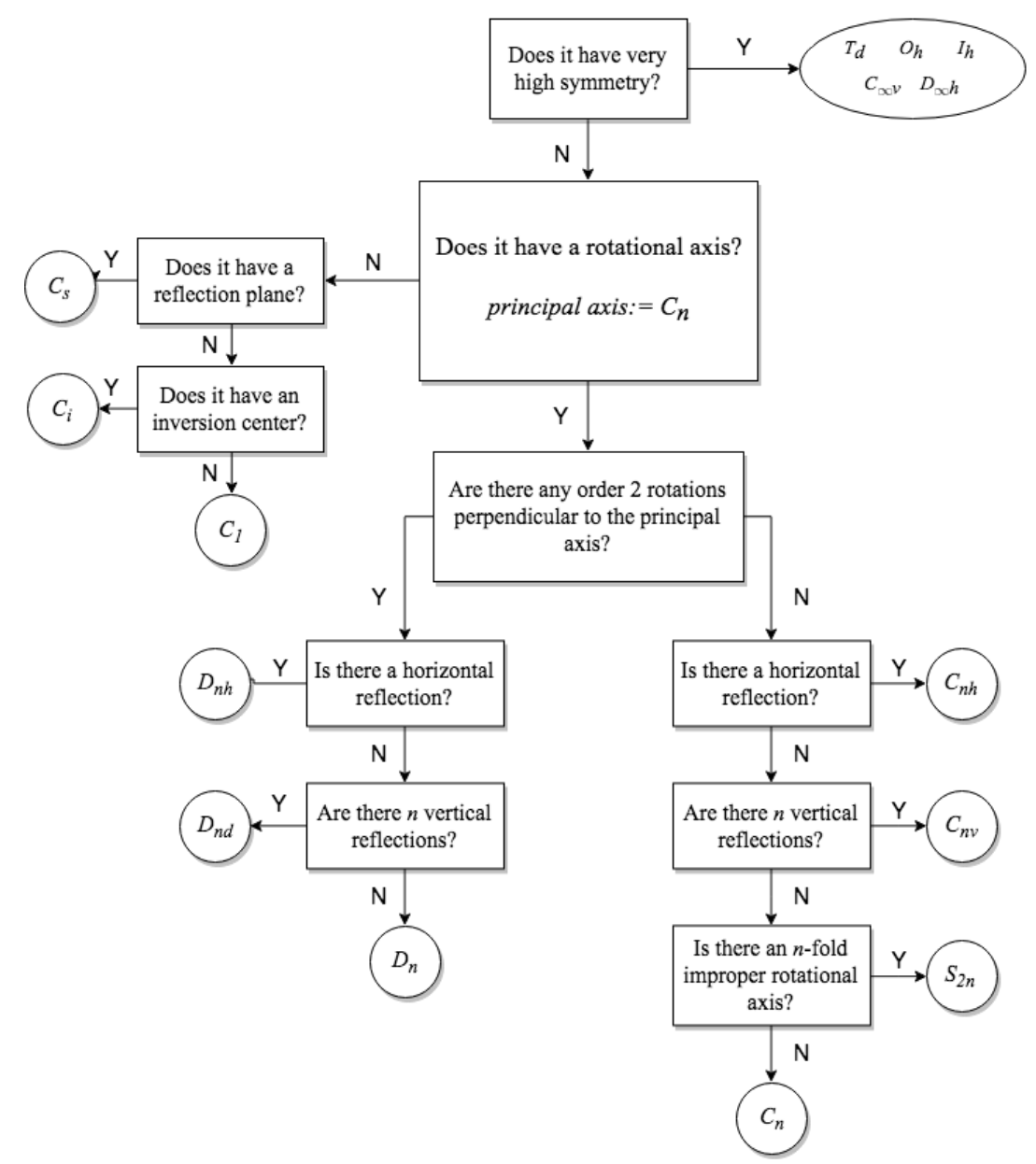

Figure 21. A typical point group classification flowchart given to inorganic chemistry students.

The groups given as outputs are described using classes of chemical groups with similar group structures, derived from the collection of symmetries within each group where the order of rotation is left as a variable. Table 5 below gives the conventional mathematical name for each chemically named group in Figure 21. 
Table 5. A full collection of chemically relevant symmetry groups represented with both mathematical group names and chemical group names

\begin{tabular}{lcc}
\hline \multicolumn{1}{c}{ Group Structure } & Chemical Group Names & Mathematical Group Names \\
\hline Non axial & $C_{i}, C_{5}$ & $\mathbb{Z}_{2}$ \\
\hline Cyclic & $C_{1}, C_{2}, C_{3}, C_{4}, C_{5}, C_{6}, C_{7}, C_{8}$ & $\mathbb{Z}_{n}: n \leq 8$ \\
w/ horizontal plane & $C_{2 h}, C_{3 h}, C_{4 h}, C_{5 h}, C_{6 h}$ & $\mathbb{Z}_{2} \times \mathbb{Z}_{n}: 2 \leq n \leq 6$ \\
w/ vertical planes & $C_{2 v}, C_{3 v}, C_{4 v}, C_{5 v}, C_{6 v}, C_{7 v}, C_{8 v}$ & $D_{2 n}: 2 \leq n \leq 6$ \\
\hline Dihedral & $D_{2}, D_{3}, D_{4}, D_{5}, D_{6}, D_{7}, D_{8}$ & $D_{2 n}: 2 \leq n \leq 6$ \\
w/ horizontal plane & $D_{2 h}, D_{3 h}, D_{4 h}, D_{5 h}, D_{6 h}, D_{7 h}, D_{8 h}$ & $\mathbb{Z}_{2} \times D_{n}: 2 \leq n \leq 6,8$ \\
w/ vertical planes & $D_{2 d}, D_{3 d}, D_{4 d}, D_{5 d}, D_{6 d}, D_{7 d}, D_{8 d}$ & $D_{2 n}: 2 \leq n \leq 6$ \\
\hline Improper rotations & $S_{4}, S_{6}, S_{8}$ & $\mathbb{Z}_{4}, \mathbb{Z}_{6}, \mathbb{Z}_{8}$ \\
\hline Cubic groups & $T, T_{h}, T_{d}, O, O_{h}, T_{h}, I, I_{h}$ & $A_{4}, A_{5}, S_{4}, A_{4} \times \mathbb{Z}_{2}, A_{5} \times \mathbb{Z}_{2}, S_{4} \times \mathbb{Z}_{2}$ \\
\hline Linear groups & $C_{\infty \infty}, D_{\infty h}$ & $S O_{2}, O_{2}$ \\
\hline
\end{tabular}

While one might initially assume that this algorithm is a classification of the symmetry groups of three-dimensional shapes, because molecular models are 3dimensional objects, it is quite different from what a mathematician might create if they were solely interested in classifying the symmetry groups of 3-d figures. Instead, this algorithm is a classification of a restricted set of symmetry groups of 3-d figures. The biggest restriction on this classification system comes from chemistry, where the order of any rotation must be less than or equal to 8 . This is because the atoms in a molecule need to be close enough to form electrical bonds which maintain the stability of the molecule. This leaves only 28 symmetry groups up to isomorphism within the classification system.

Further, the notion of equating isomorphic groups is not utilized by chemists when classifying symmetry groups. For a mathematician a symmetry group generated by a sole order 2 rotation and a different symmetry group containing only a reflection are both isomorphic to any group of order two, and therefore quickly considered "equivalent, up to isomorphism" as their algebraic structures are identical. This family of isomorphic groups is typically identified with the specific group $\mathbb{Z}_{2}$ (integers under addition mod 2). 
In contrast a chemist would consider these two different groups, $C_{2}$ and $C_{s}$ respectively, because the kind of symmetry the molecule possesses has different chemical consequences such as differences in modes of vibrations or bonding capabilities.

Therefore the 32 symmetry groups up to isomorphism included in the classification algorithm are represented by 51 different chemical point groups. A consequence of having isomorphic groups with different group names, is that when re-considering the flowchart in Figure 21 written with conventional math group names in place of the chemical counterparts there are multiple terminal branches with the same group structure, as seen in Figure 22 below.

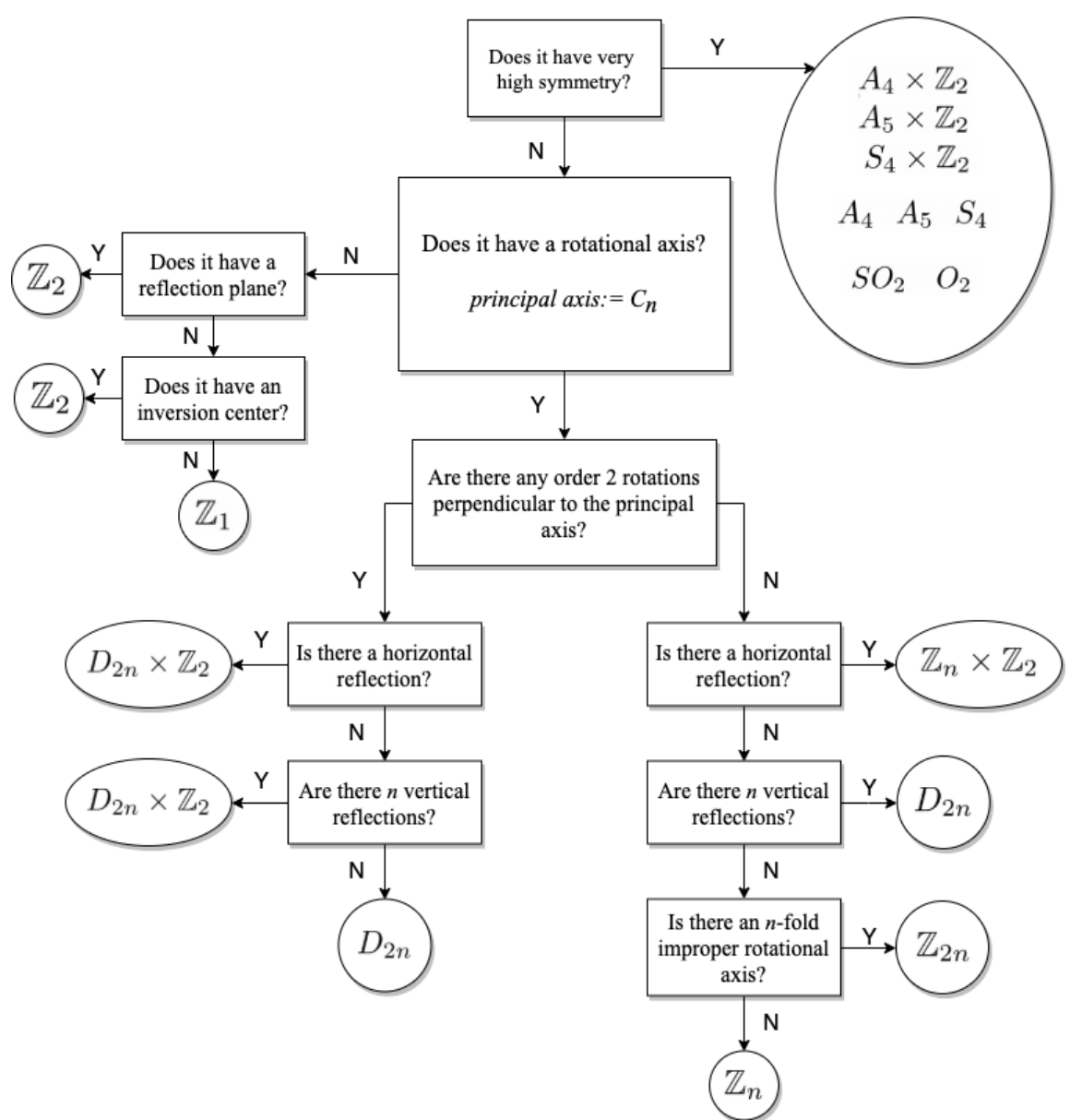

Figure 22. A typical flowchart given to inorganic chemistry students with mathematical group names instead of chemical group names 
One last difference between the classification of chemically important symmetry groups by chemists and the classification of symmetry groups of 3-d figures by mathematicians is which symmetries are considered primitive. As described above in Table 4 chemists use four flavors of symmetries: rotations, reflections, inversions, and improper rotations. Mathematicians on the other hand only consider rotations, reflections, and inversions to be the primitive symmetries in 3-space as improper rotations can be generated by rotations and reflections. So ultimately what I am trying to support the student reinvention of is a mathematical version of a similar classification of chemically important symmetry groups using conventional mathematical names and notions as opposed to those employed by chemists. Therefore, the overall goal of this study was to design a local instructional theory for how students might use group theory in an experientially real way to classify chemically important symmetry groups. This led to main research question: How can students be supported in reinventing an algorithm for the classification of chemically important symmetry groups?

Theoretical Perspective

For this study I chose to conduct a design experiment using the instructional design theory known as, realistic mathematics education (RME). RME has both a theoretical perspective and three accompanying design heuristics that helped guide this study. The notion that mathematics is a human activity is at the very core of RME and provides the theoretical perspective that the goal of an instructional sequence is to provide students with an opportunity to participate in the organizational activity of mathematizing. The first design heuristic of RME that significantly influenced this research is notion of 
guided reinvention. Gravemeijer and Doorman (1999) explain that the idea of guided reinvention "is to allow learners to come to regard the knowledge that they acquire as their own private knowledge, knowledge for which they themselves are responsible" ( $p$. 116). The second design heuristic, didactic phenomenology, emphasized the need to provide the students with a context that could be productively mathematized using exactly the mathematics they are meant to learn. In other words, in order to have the students reinvent a way to identify group structures for molecules they first needed a situation in which they determined group structures.

The third and final RME design heuristic that guided this work was the notion of emergent models. The emergent models heuristic is used in the creation of an LIT as, "a tool for conceptualizing how students' informal mathematical activity can emerge from a starting point context and then develop into the more formal mathematics that is the goal of instruction" (p.26, Larsen, 2018). As the students begin to mathematize the given problem situation their initial strategy emerges as a model of the students' activity. Once the students' strategy is leveraged in new situations it evolves into a model for more formal activity. Gravemeier (2002) describes three interrelated mechanisms of emergent models: "Firstly, there is the overarching model, which first emerges as a model of informal activity, and then gradually develops into a model for more formal mathematical reasoning. Secondly, the model-of/model-for transition involves the constitution of some new mathematical reality - which can be called formal in relation to the original starting points of the students. Thirdly, in the concrete elaboration of the instructional theory, there is not one model, but the model is actually shaped as a series of symbolizations" 
(Gravemeijer, 2002). In this sense, this heuristic is used to describe both the qualities and features of the process, not just the process itself.

A local instructional theory (LIT) describes a generalized roadmap for student reinvention of a particular mathematical concept (Gravemeijer, 1998). This path is generalized sequence of steps, described in terms of student strategies and ways of thinking that have been identified as important milestones in the development of the fundamental ideas of the particular mathematical concept (Larsen \& Lockwood, 2013). The theory is local in the sense that it describes how the specific topic should be taught to fit the guiding principles of RME. However, the LIT differs from just a sequence of instructional tasks in the sense that the LIT also focuses on the rationale in choosing such a sequence of activities. The rationale provides an explanation of how the particular instructional activities comply with the intention to give students the opportunity to reinvent mathematics (Gravemeijer, 1998). More importantly, the rationale also explains how the instructional activities support students in their reinvention. The LIT described here is offered in terms of students' mathematical activity coupled with suggestions on how to both evoke and leverage such activity, followed by examples of students work collected in the design study.

The local instructional theory described here represents $a$ path in which students may reinvent a classification system for determining group structures from given molecules. The specific mathematical activity of the students may vary depending on their previous mathematical background, particularly their prior exposure to group theory. The LIT described here is the culmination of refinement and testing with various individuals but 
does not exhaust all approaches or paths which students may take in the reinvention process.

Methods

The local instructional theory described in this paper is the result of a design experiment (Collective, 2003). Design experiments provide an opportunity to systematically study the various forms of learning within the context and the means of supporting them; coupled with the pragmatic bend of engineering a particular form of learning through a built-in refinement method (Cobb, Confrey, DiSessa, Lehrer, \& Schauble, 2003). By conducting three different teaching experiments, I was able to iteratively test and refine the LIT, ultimately abstracting the key steps from multiple observations of students' mathematical activity. Details about the details and duration of each experiment can be found in Table 6 below. All sessions were audio and video recorded, all written work was collected, and all of the participants were compensated for their time. Each of the teaching experiments were conducted with a pair of mathematics students who had no apriori experience with group theory in the context of chemistry. While none of the participants had any formal training in chemistry, the mathematical backgrounds of the participants varied across the three experiments.

Table 6. An overview of each of the three teaching experiments.

\begin{tabular}{|l|l|l|}
\hline \multicolumn{1}{|c|}{ TE1 } & \multicolumn{1}{c|}{ TE2 } & \multicolumn{1}{c|}{ TE3 } \\
\hline Emmy \& Felix & Arthur \& Stu & Ada \& Sophie \\
\hline 4 Sessions & 12 Sessions & 11 Sessions \\
\hline $60-80 \mathrm{~min} /$ session & $45-120 \mathrm{~min} / \mathrm{session}$ & $45-90 \mathrm{~min} / \mathrm{session}$ \\
\hline $\begin{array}{l}\text { 2 Graduate students in } \\
\text { mathematics education }\end{array}$ & $\begin{array}{l}\text { 2 Undergraduates in } \\
\text { mathematics }\end{array}$ & $\begin{array}{l}1 \text { electrical engineering major } \\
1 \text { mathematics major }\end{array}$ \\
\hline
\end{tabular}


Ample group theory experience; including prior graduate level mathematics experience classifying finite groups
Both had completed an introductory group theory course the previous term
No previous group theory or proof-based mathematics course

The goal of the first teaching experiment (TE1) was to explicate $a$ way in which a pair of students could successfully classify chemically important point groups and so it served as a kind of an existence proof (for more detail, see Bergman \& French, 2019). In order to learn how students might reinvent a classification system for symmetry groups, TE1 was conducted with a pair of mathematics education graduate students, Emmy and Felix, each of whom had completed a graduate level course focused on classifying groups of finite order. The following teaching experiments in the study were conducted with pairs of undergraduates. The second teaching experiment (TE2) was conducted with a pair of undergraduate mathematics students, Arthur and Stu, who had recently completed a traditional quarter long lecture-based 300-level introductory group theory course. The final teaching experiment (TE3) was conducted with a pair of undergraduate students, Ada an electrical engineering student and Sophie a mathematics student, neither of whom had any experience with group theory. Ada and Sophie had both recently completed a 200-level linear algebra course and were provided two days of selected lessons from the Inquiry Oriented Abstract Algebra (IOAA) curriculum (Larsen, 2010a) as an introduction to group theory prior to implementing the local instructional theory outlined below.

\section{An Overview of the Local Instruction Theory}

Below is a local instructional theory for the guided reinvention of a classification system for chemically important symmetry groups that can be used to identify the symmetry group of a given molecule. First I provide an overview of each step of the LIT 
in Table 7 which gives each step of the LIT accompanied with a short rationale. The LIT describes the emergence and evolution of two different emergent models. The first referred to as Model 1 describes the students' strategy for creating ever more compact representations for the group concept. Model 1 concludes with the use of some kind of representation like a group name. Model 1 then feeds into Model 2 which describes the students classification system for chemically important symmetry groups. It's important to note that while students complete the LIT reinventing a classification system for chemically important symmetry groups, they may interpret it as a tool for identifying symmetry groups, not necessarily as a classification system.

Following Table 7 is a more detailed description of each step of the LIT using examples of students work collected from each of the teaching experiments. Most of the examples of students work come from the third and final teaching experiment TE3, conducted with Ada and Sophie. The steps described below consist of the major chapters of the student's mathematical activity that encompassed the reinvention of a classification system for chemically important symmetry groups. However, it is important to note that the sequence of steps described below is not the same sequence that any one of the teaching experiments followed. During each of the teaching experiments all the steps were completed, but the order was different as the LIT was tested and refined. The sequence of steps given in the LIT above represents the mathematical activity associated with one way students might reinvent this kind of algorithm, abstracted from and informed by multiple iterations of the design research cycle. 
Table 7. A local instructional theory for the guided reinvention of a classification algorithm for chemically important symmetry groups

\begin{tabular}{|c|c|}
\hline Step & Rational/Purpose \\
\hline $\begin{array}{l}\text { 0. An experientially real } \\
\text { starting point in the } \\
\text { context of geometric } \\
\text { symmetry where } \\
\text { students are introduced } \\
\text { to the idea that a } \\
\text { symmetry can be } \\
\text { described as a rigid } \\
\text { motion that can be } \\
\text { combined with another } \\
\text { symmetry to produce a } \\
\text { (possibly new) } \\
\text { symmetry. }\end{array}$ & $\begin{array}{l}\text { The idea that a symmetry can be described as a rigid motion that can be } \\
\text { combined with another symmetry to produce a (possibly new) } \\
\text { symmetry must be experientially real to students. This is important } \\
\text { because in order to do group theory the elements of the group, in this } \\
\text { case the symmetries, must be able to be combined under some kind of } \\
\text { operation. Symmetries as physical properties, such as planes of } \\
\text { reflection or as orientations from the result of a rotation don't make } \\
\text { sense to combine. However a reflection about a plane followed by a } \\
\text { particular rotation is absolutely reasonable. Students need to also have } \\
\text { some understanding that symmetries can be collected into groups. This } \\
\text { may require preliminary instructional activities. }\end{array}$ \\
\hline $\begin{array}{l}\text { The emergence of a } \\
\text { strategy for representing } \\
\text { the symmetry group of a } \\
\text { given molecule as a } \\
\text { model-of the symmetry } \\
\text { group of a particular } \\
\text { molecule (Model 1) }\end{array}$ & $\begin{array}{l}\text { The reinvention of a classification system for determining symmetry } \\
\text { groups of various molecules should begin with students' informal } \\
\text { activity in an experientially real context. The students initial process for } \\
\text { identifying and describing the symmetry elements of the molecules as a } \\
\text { group (Model 1) can be seen as anticipating the process that they will } \\
\text { eventually formalize and incorporate into something like the standard } \\
\text { flow chart (Model 2). This activity should anticipate both the } \\
\text { uniqueness of a symmetry group for each molecule based on the } \\
\text { collection of symmetries that exist within the molecule, and it should } \\
\text { expose students to a variety of group structures; such as } \mathbb{Z}_{2} \times \mathbb{Z}_{2} \text {, a } \\
\text { dihedral group, and the direct product of a dihedral group and } \mathbb{Z}_{2} \text {. At } \\
\text { this point the emergence of a system to determine symmetry groups, } \\
\text { (Model 2) is only seen by the researcher as a model of the student's } \\
\text { informal activity related to identifying particular symmetry groups. }\end{array}$ \\
\hline $\begin{array}{l}\text { 2. Describing and naming } \\
\text { the symmetry group with } \\
\text { an encapsulated group } \\
\text { representation as a } \\
\text { model-for the symmetry } \\
\text { group of a molecule } \\
\text { (Model 1) }\end{array}$ & $\begin{array}{l}\text { As students begin to develop a system for identifying various symmetry } \\
\text { groups, they need to decide on a group representation that is } \\
\text { encapsulated or compact enough to be used as the outputs for their } \\
\text { classification system. The students' encapsulated group representation } \\
\text { can be seen as the final version of the students' model for their unique } \\
\text { symmetry group (Model 1), which again will be incorporated into their } \\
\text { classification system (Model 2). This is an initial step in the transition } \\
\text { from model of to model for. }\end{array}$ \\
\hline $\begin{array}{l}\text { 3. Formulating an explicit } \\
\text { system for determining } \\
\text { the symmetry group of a } \\
\text { molecule (Model 2) as } \\
\text { mathematizing the } \\
\text { previous activity }\end{array}$ & $\begin{array}{l}\text { A system for determining the unique symmetry group for a given } \\
\text { molecule, which initially emerged as a model of the students' informal } \\
\text { activity, is explicated and then used as a model for more formal activity } \\
\text { as it feed into a classification system for determining the symmetry } \\
\text { group of any given molecule (Model 2). }\end{array}$ \\
\hline
\end{tabular}




\begin{tabular}{|c|c|c|}
\hline & $\begin{array}{l}\text { "Applying" the system } \\
\text { for determining the } \\
\text { symmetry group of a } \\
\text { specific molecule } \\
\text { (Model 2) in similar } \\
\text { situations }\end{array}$ & $\begin{array}{l}\text { The transition of a classification system (Model 2) from a model of the } \\
\text { students' activity to a model for more formal reasoning can begin by } \\
\text { having the students consider similar contexts by investigating more } \\
\text { molecules. Students can be given a variety of molecules whose } \\
\text { symmetry groups are both the same and slightly different than what } \\
\text { they saw in Step } 1 \text {. By considering new molecules with very similar } \\
\text { group structures to the ones they have already investigated, say } \mathrm{D}_{8} \times \mathbb{Z}_{2} \\
\text { instead of } \mathrm{D}_{6} \mathrm{X} \mathbb{Z}_{2} \text {, students can test their system. By considering } \\
\text { something new, such as a pure cyclic group, students can also refine } \\
\text { their system to include more group structures. }\end{array}$ \\
\hline & $\begin{array}{l}\text { "Applying" the system } \\
\text { for determining the } \\
\text { symmetry group of a } \\
\text { specific molecule } \\
\text { (Model 2) in different } \\
\text { contexts }\end{array}$ & $\begin{array}{l}\text { This transition of the classification system to a model of a classification } \\
\text { system for chemically important symmetry groups (Model 2) which can } \\
\text { be used for more formal reasoning can continue by having the students } \\
\text { consider different but structurally similar contexts. This is } \\
\text { accomplished in two different ways: } \\
\text { a. Students are first asked to test their model on a molecule that has a } \\
\text { symmetry group that students already have experience with, but which } \\
\text { can be generated by new kind of symmetry that they have not } \\
\text { experienced, an inversion. In doing so, students can continue to refine } \\
\text { their model to include more kinds of symmetries and/or more kinds of } \\
\text { symmetry groups. } \\
\text { b. Students are then asked to test their model on very symmetric } \\
\text { molecules with high order symmetry groups. While these types of } \\
\text { molecules have new symmetry groups than those the students have } \\
\text { previously experienced, they are composed of the same symmetry } \\
\text { elements the students have already used in their model. }\end{array}$ \\
\hline & $\begin{array}{l}\text { Justifying generality / } \\
\text { robustness of the } \\
\text { classification system } \\
\text { (Model 2) }\end{array}$ & $\begin{array}{l}\text { Students should know that one aspect of a complete classification } \\
\text { system is one in which they can convince someone else that their } \\
\text { flowchart can accurately identify the symmetry groups of any three- } \\
\text { dimensional molecule. Students' arguments for the generalizability of } \\
\text { their classification system may be quite different depending on their } \\
\text { level of mathematical background. }\end{array}$ \\
\hline
\end{tabular}

Step 0: Establishing geometric symmetry as an experientially real starting point.

The main LIT assumes that there is an experientially real starting point, where the students can be given physical models of a variety of molecules to investigate the unique symmetry group of each. Students should have ample time to establish the context of molecular structures as personally meaningful, and to help build up their informal understanding of how someone could investigate three-dimensional structures. For some students working directly with ball and stick models of molecules is experientially real 
enough to begin considering symmetries as rigid motions such as Emmy and Felix in TE1 whose approach is described in Bergman \& French, 2019. However, the context of investigating symmetries in three-dimensional space may not be experientially real for all students. Depending on the mathematical background of the students it may be necessary to start with a preliminary instructional activity to help establish the idea that a symmetry can be described as a rigid motion that can be combined with another symmetry to produce a (possibly new) symmetry. It is also important that students understand that two symmetries that yield the same resulting orientation are considered equivalent. Lastly, students need to be aware of the notion of collecting symmetries into a group structure, i.e. a symmetry group. Therefore, students need to be cognizant of the group concept.

\section{Sample Tasks and Prompts for Step 0:}

The activity of the students in TE2 gives a compelling argument for the need of some kind of preliminary task to support students as they begin identifying 3-dimensional symmetries. The students in TE2 began the teaching experiment by exploring threedimensional ball and stick models with the following prompt. It was clear from the beginning of the session that the students weren't sure what counted as a symmetry.

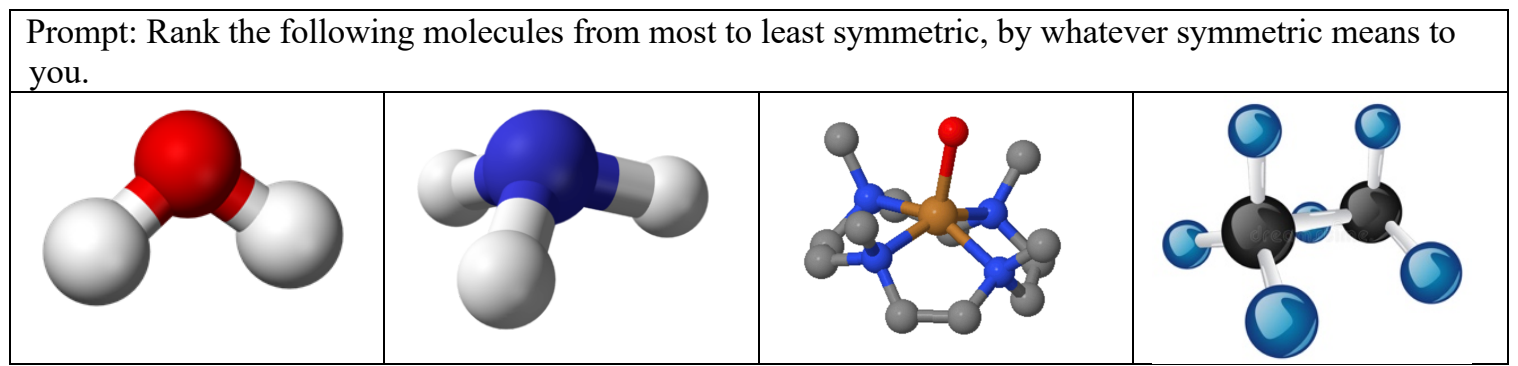

Figure 23. The initial prompt given to participants in TE2 
From the very beginning it was pretty clear that Arthur and Stu did not have robust conceptions of symmetry, or at least they were not able to readily use their conception of symmetry to easily rank the molecules. Arthur and Stu had various ideas related to symmetry that they tried to use to rank the molecules, but even they admitted that these ideas were "super rough":

Arthur: (picks up water) ...least symmetric, I suspect right out of the gates.

Stu: This one (ethane) feels the most symmetric because it has several axes. Arthur: Umm, several axes is good.

Annie: Why did you feel like (water) was least symmetric?

Arthur: Um, the thing that jumped out is the angles here (in water) I mean I can't think of a... looking at this angle I can think of it being symmetric across this line (Figure 24) but I don't see a lot of other opportunities to do that. I mean by my super rough definition of symmetry, I just see, ah hmm.

Yeah I don't have much to follow up on that. Multiple axes was helpful on that one (ethane).

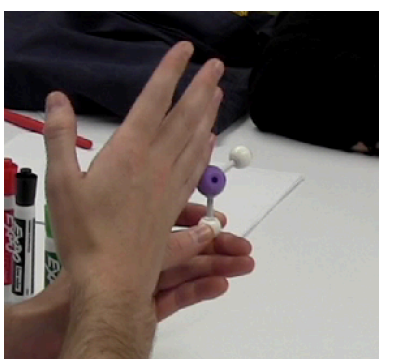

Figure 24. Arthur's gesture indicating a reflection plane in water.

Stu: I guess this one (water) seems more symmetric to me than this one (ammonia) because I see this way (Figure 25a) and I didn't see this way 
until you mentioned it. And on this one (ammonia) I only see one way, this tri-symmetry.

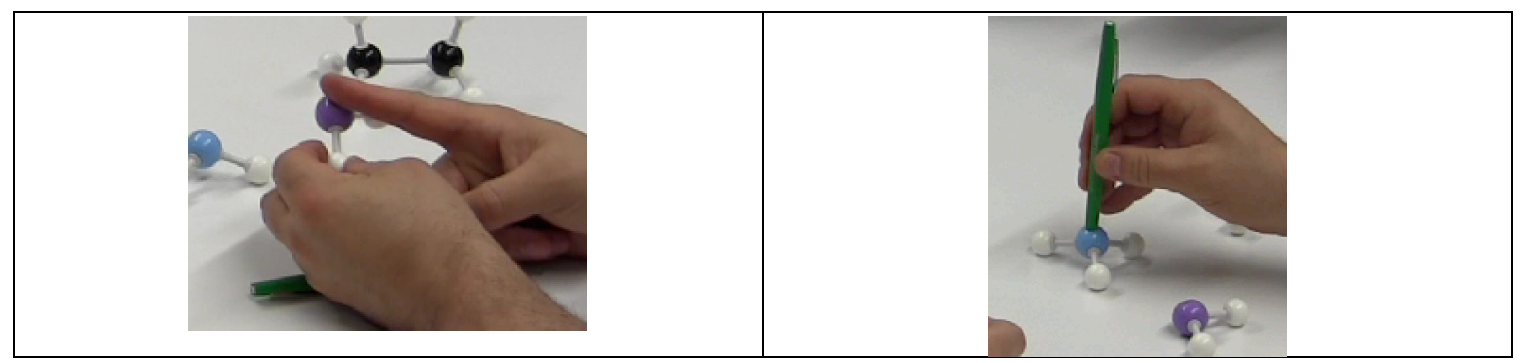

Figure 25. Stu indicating rotational axes in water.

Annie: What are you seeing? What are you basing who has more symmetries on?

Stu: Yeah, um I see two axes of symmetry on this one (water) but I only see one on this one, and that's what I'm basing it off of.

Annie: Can you kind of point to where you see the axes of symmetry?

Stu: Yeah on this one ammonia I see it right here (Figure 25b) and on this one (water) I see it, (Figure 25a) well you could rotate it all the way around it like that and then, as Arthur said, this way. Ah I don't see anymore.

Arthur: Maybe I was just reaching for (ammonia), looking at, maybe I was thinking more about rotational symmetries. I don't actually know if that's a thing.

When I turned to ask Stu how he was thinking about symmetries he said, similarly to Arthur, and as he was answering he said just noticed another axis of symmetry in ammonia (see Figure 26 below). Assuming he meant a rotational axis, I asked him to elaborate and we had the following exchange: 
Annie: So now when you say there is a rotational axis here, are you imaging kind of like rotating around this bond? Are you seeing the axis go through these guys (one hydrogen and the center atom)?

Stu: No, I'm not seeing a rotational axis I'm seeing a mirror axis a reflection. Annie: ok so maybe more like a plane?

Stu: Yeah so if there were a line here (Figure 26) then there's definitely symmetry mirroring across that line. And if you rotate it, it twice, but that's what I'm talking about.

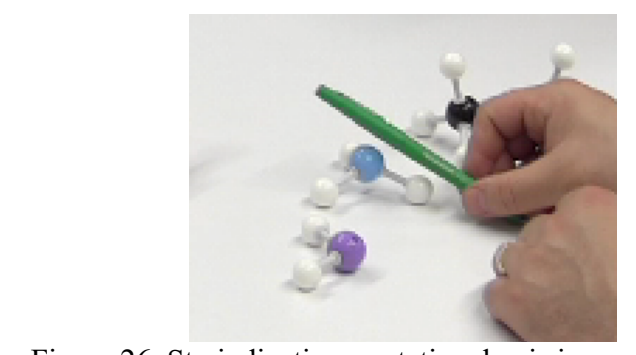

Figure 26. Stu indicating a rotational axis in ammonia.

Stu's language shows that he is trying to use the properties of two-dimensional symmetries for three-dimensional motions, for example when Stu mentions "mirroring across a line." While he has some of the right parts, at this point it seemed to be clear that Stu's notion of symmetry is intimately tied to 2-dimensional space. Shortly after, Stu then mentioned that he hadn't started looking for 'diagonal reflections', and when I ask him to elaborate what he means by 'diagonal reflections' he said:

Stu: ...like where you would... it's easier with a piece of paper, it's hard for me to see it with 3-dimensions. Normally I would think of this sort of thing (Stu rotates his hand back and forth as seen in Figure 27 below) along a diagonal, but I don't really see that here. 


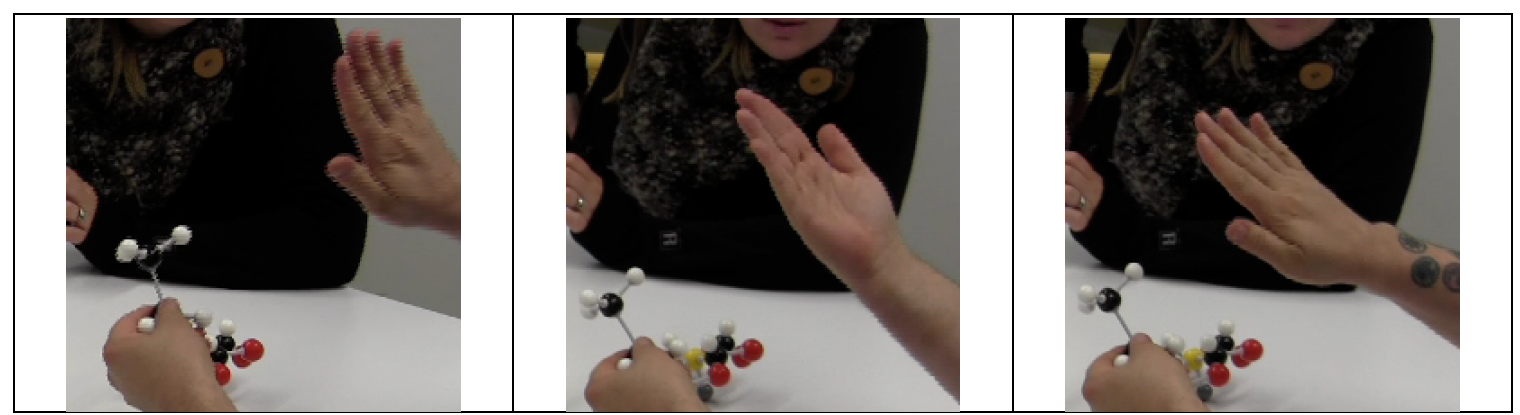

Figure 27. Stu demonstrating a reflection with a hand gesture.

This explanation shows that Stu's idea of reflection is consistent with a 3-dimensional rotation. While Stu is correct in his thinking that there exists an equivalence between 2dimensional reflections and 3-dimensional rotations, this idea of reflection is not productive when trying to identify 3-dimensional reflections.

This attention to rotational symmetry persisted throughout the entire session and eventually fed into their notion of symmetry in general. Eventually Stu suggests the following idea for how he's thinking about symmetry; for Stu one molecule is more symmetric than another if it has "more ways to look similar". Stu gives an example saying, "there is a higher proportion of time that ethane will get back to itself." Arthur elaborates on this thinking by saying:

Arthur: If I'm looking at this in like a chemistry way, where like at any one point this thing (Tetra-aza Copper II, see Figure 28) has just randomly been rotated, in some, some configuration here, and we're just constantly having some random rotation or something like, this thing (ethane) seems much more likely to end up in a symmetrical position. Like if they're both rotating the same frequency (see Figure 28), we're more likely to get this (ethane) back to a starting point then this (Tetra-aza Copper II). Whereas 
like this (Tetra-aza Copper II) would arrive back at starting configuration less frequently.

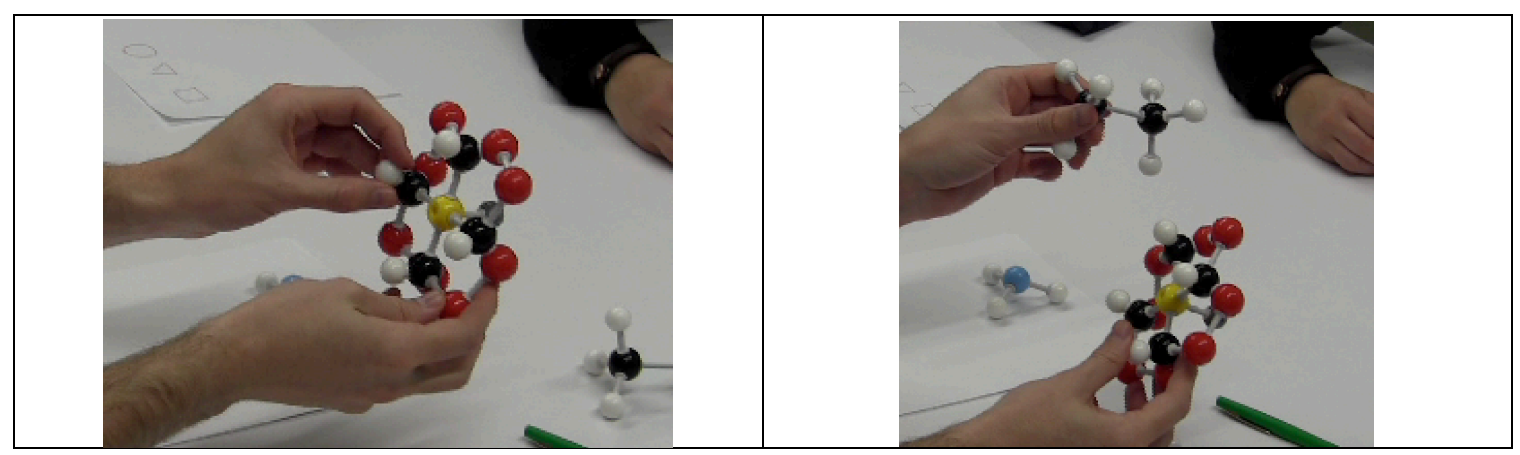

Figure 28. Arthur using ball and stick models of Tetra-aza Copper II (left) and ethane (right) to describe his idea of symmetry.

Notice that this notion of symmetry only attends to rotational symmetry, which can be physically performed on ball and stick molecules but fails to include any other kinds of symmetry such as reflections which are strictly mental operations that cannot be performed physically. Stu's description of reflection and the students' dependence on rotations in TE2 suggested that future students could probably benefit from a conversation about symmetries before they're asked to consider three-dimensional models.

Stu and Arthur reinforced my findings from TE1 that beginning an investigation of symmetries in three-dimensional space is non-trivial for students (Bergman \& French, 2019). Starting with a preliminary investigation of two-dimensional symmetries has two advantages, first it provides students with an opportunity to consider and discuss symmetries as objects, as opposed to a property of a figure. The second advantage of starting with a preliminary investigation of two-dimensional symmetries it that allows students to develop definitions of both symmetry and symmetry equivalence before considering three-dimensional molecules. When considering physical models of 
molecules students often gravitate towards rotations and struggle with initially accepting reflections in particular, presumably because rotations are the only symmetries that can actually be "performed" on a physical model. However, if students are armed with a definition of symmetry that counts any distance preserving map that takes the object to itself and have already considered reflections in two-dimensional space, they seem much more comfortable with accepting and identifying reflections when considering threedimensional figures. In other words, students are most successful when they commit to symmetries as motions. In order to support Stu and Arthur's reinvention of these definitions I began the next session with The Measuring Symmetry Task (Larsen \& Bartlo, 2009) which had been shown powerful in promoting student generated definitions of both symmetry and symmetry equivalence. Once they had a chance to explore symmetries in 2-dimensions they were much more comfortable returning to 3-dimensions to start considering symmetry groups.

Learning from this experience I decided to begin TE3 with the measuring symmetry task. The students in TE3 completed the task and created definitions for both symmetry and equivalence of symmetries. For more detail on the measuring symmetry task and the mathematical activity it elicits, see Larsen \& Bartlo, (2009). Since the students in TE3 had no previous experience with group theory they were provided additional preliminary activities beyond the measuring symmetry task. In order for Ada and Sophie, the students in TE3, to engage in in identifying symmetry groups, they needed to be aware of symmetry groups. To introduce them to the group concept I supplied them with three sessions of instruction where they reinvented the group concept using the IOAA curriculum (IOAA Citation). For a detailed description of the mathematical activity of 
students reinventing the group concept, see $A$ local instructional theory for the guided reinvention of the group and isomorphism concepts (Larsen, 2013).

Step 1: The emergence of a strategy for representing the symmetry group of a given molecule as a model-of representing the symmetry group of any molecule (Model 1)

Once an experientially real starting point has been established in the context of a three-dimensional symmetry investigation, the first step in the reinvention process is the emergence of a strategy for describing the symmetry group of a given molecule (Model 1). This strategy will emerge as a model of the students' activity as they identify the symmetry groups of a collection of molecules and includes two parts:

1. Identify the set of symmetries

2. determine the group structure

I used the prompt in Figure 29 below to evoke this activity.

Sample Tasks and Prompts for Step 1:

Prompt: Find the symmetry group for each of the following:

(2)

Figure 29. The initial prompt given to students in TE3.

In each of the teaching experiments I began by asking students to find the symmetry groups of water, ammonia, and ethane in an eclipsed configuration. In every experiment the students began with water, followed by ammonia, and lastly ethane. 


\section{Step 1: Part 1. Identify the set of symmetries}

Students often start with an exploration of the rotations, followed by reflections; they seem to gravitate towards rotations first as they are motions that can be physically performed on the model. As students identify various symmetries, they begin to create inscription systems, typically starting with geometric images to record their findings.

In TE3, students started by deciding on an inscription system to keep track of each symmetry as they explored all the possible orientations that can result after a symmetry is applied to a fixed starting orientation. The students also associated these orientation pictures with symbols that identified them with the symmetry operations as seen in Figure 30 below. Similar to students in TE1 and TE2 Ada and Sophie often associated the highest order rotation with the letter $R$. Ada and Sophie then went a step further and also fixed their notion of rotation to the $z$-axis. With the exception of water they used $F$ to represent a reflection through the $x y$-plane, conventionally called a vertical reflection whose mirror plane contains the principal axis. Their choice of $F$ for a reflection was a relic from their work with reflections in two-dimensions which they labeled $F$ for "flips." Lastly, Ada and Sophie used the term "transport" to represent a horizontal reflection, one whose mirror plane is orthogonal to the principal axis. This choice of $T$ represented their idea that reflections were kind of similar to transporters in Star Trek. Transporters are a fictional teleportation machine which convert a person or object into energy pattern then beamed it to a target local where it rematerialized. The use of symbols is an important step in mathematizing the symmetries to the point where their combinations could be easily recorded, in other words their use allows for easier recordkeeping of more formal mathematics. 


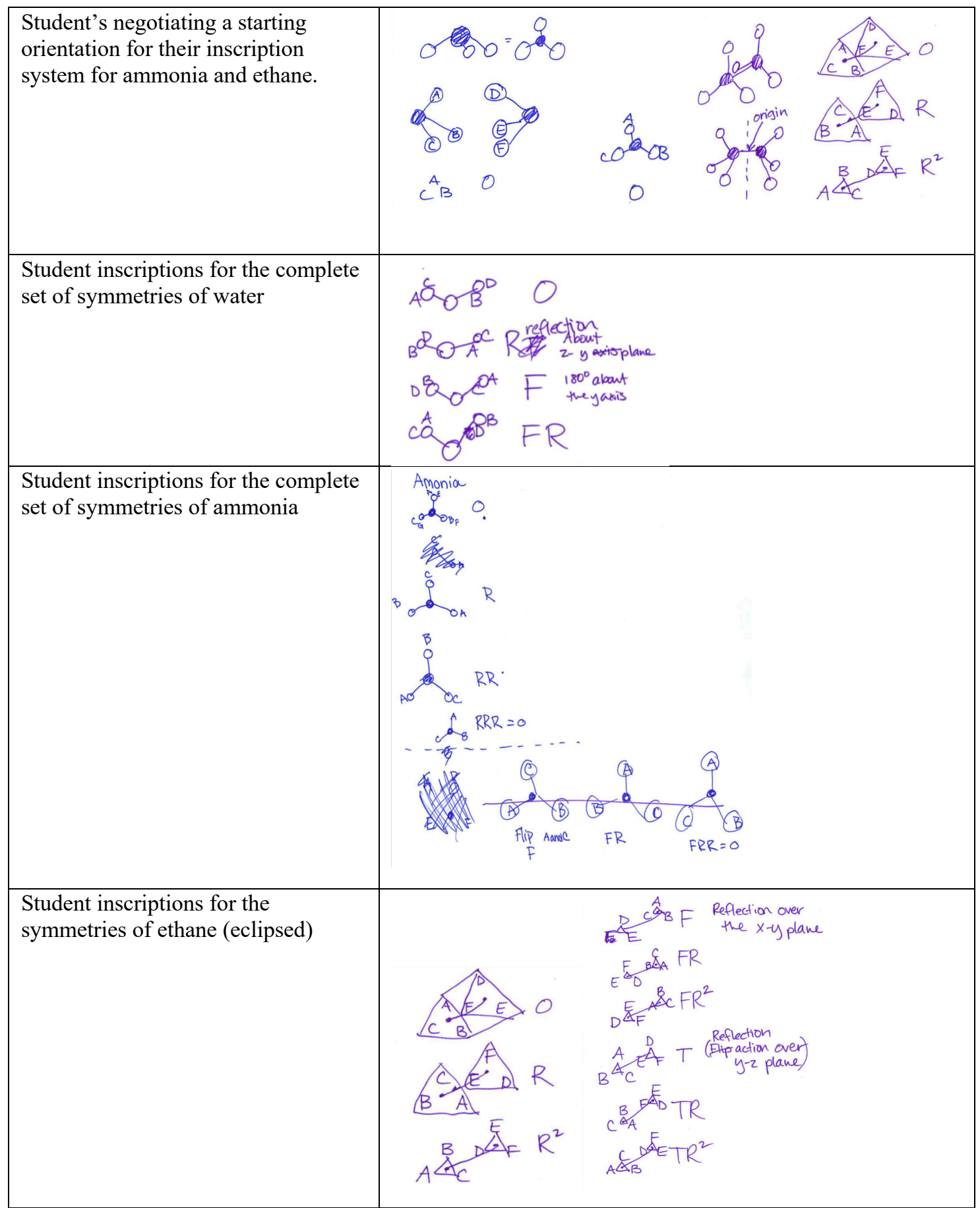

Figure 30. Students' geometric representations of symmetry group elements for water, ammonia, and ethane from TE3.

Sometimes students used a Cayley table as an organizational tool with the intention of

find more symmetries. For example, in TE2 Arthur and Stu created a Cayley table for 
ethane after finding eight different symmetries aware that there may be more symmetries as seen in their response to my asking them if they thought they had them all as we transitioned to creating the Cayley table in Figure 31:

Annie: Do you think you have all the symmetries so far?

Stu: I'm not convinced we've discovered them all.

Annie: Do you think there might be more once we start combining them together?

Stu: I'm open to the idea, that's for sure.

Arthur: So I was thinking when we look at the symmetries of like a square, we've got our rotations and our reflections, but we can't like twist it or break it or something. And I feel like now we're actually starting to see the, ya know the state results of these three (hydrogen) needing to stay within these three (hydrogen). That like, we can't, there's no symmetry that like swaps these two (hydrogen) but not these two (opposing hydrogen) or something. So I bet we'll see that this is just like adding one more transformation to spinning triangles or something. Which it might just be that, that this is like, we might have twice as many states as this (ammonia) or something. I bet that there's some cool stuff that we'll figure out. But I don't have any established value to add.

Stu: Yeah, it feels complete, but I wouldn't be shocked if it weren't. 
As expected, as Arthur and Stu began to fill in the body of their Cayley table for ammonia with the resulting combinations of symmetries, they began to discover new elements in the body of their Cayley table that were not part of the original eight symmetries included in the header. Stu puts red boxes around their new elements as seen in Figure 31 below.

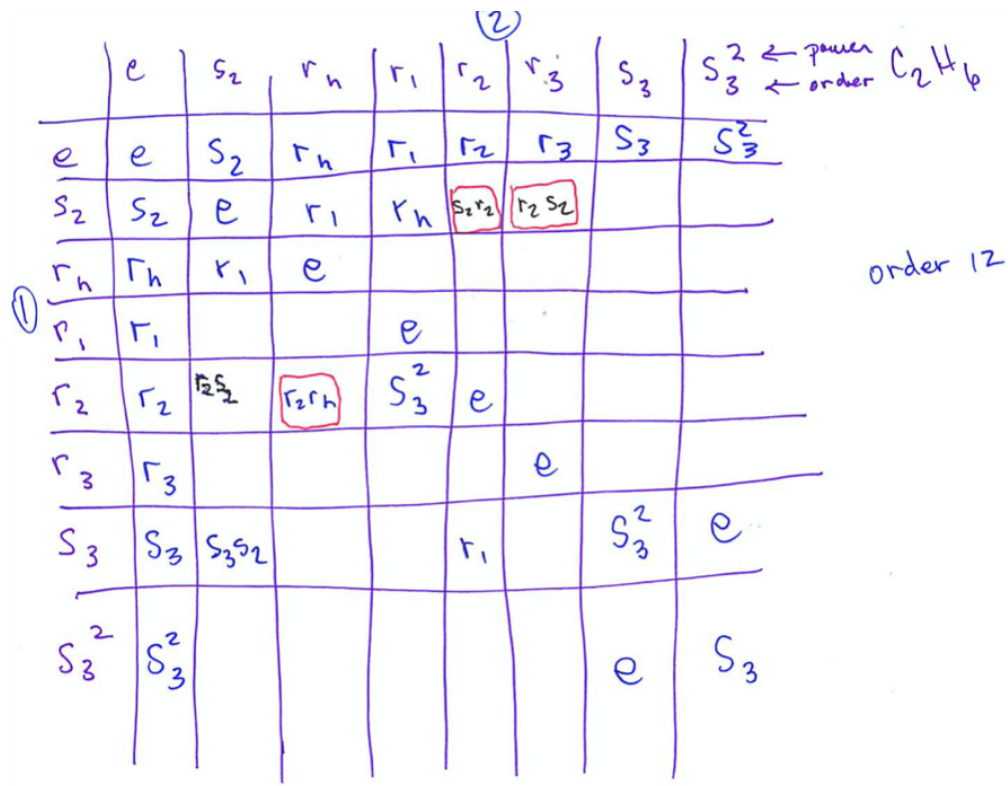

Figure 31. Arthur and Stu's initial Cayley table for (a subset of) the symmetries of ethane used to discover new symmetry elements.

Cayley tables actually played a dual role during the teaching experiments. As seen above, sometimes they were used to complete part 1 of Step 1, identifying the set of symmetries and sometimes Cayley tables were used for the part 2 of Step 1, determining the group structure.

\section{Step 1: Part 2. Determine the group structure}

In TE1 after Emmy and Felix had discovered and recorded geometric images for a few of the symmetries of ethane (in the eclipsed configuration) they quickly began to 
construct a Cayley table to determine generator relations as seen in the following exchange:

Felix: I'm gonna build a table of all of these (symmetries).

Emmy: Good, will you please keep track of this? What happens when we do $R$ with $S$ ? Can we just deal the combinations?

Felix: Let's do it.

Emmy: Cool.

While Emmy and Felix did end up using their Cayley table for ethane to discover new symmetry elements, they were always more interested in determining generator relations, and thus group structure. This can be seen in the final version of their table for ethane found in Figure 32 below. Notice that Felix and Emmy stopped using the table after they had determined the order of each generator and the generator relations as seen written above their table. This was before they had found all the elements of the symmetry group; as evidence, there are only 11 symmetries listed in the header of the table and twelve in the symmetry group. 


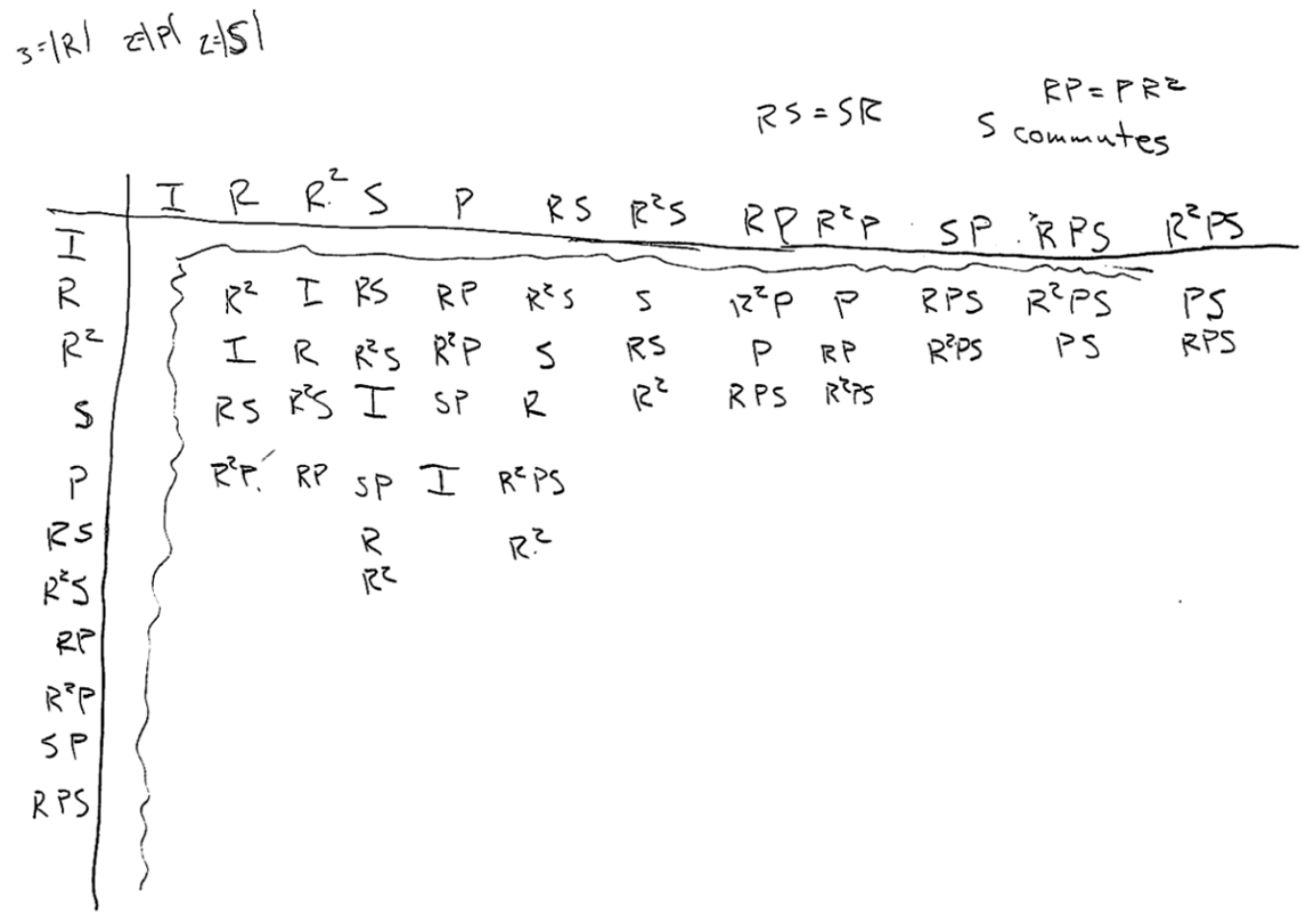

Figure 32. Emmy and Felix's Cayley table for ethane (in an eclipsed configuration).

By asking students to describe the unique collection of symmetries for each of these three molecules and organize them in a manner that helps them to determine what kind of group structure they will produce, students begin to develop a method for determining symmetries. This method for determining symmetries is the initial emergence of the students' model of a system for classifying symmetry groups (Model 2) that can be used to determine the symmetry group of a given molecule. Asking students to investigate the variety of group structure represented by water, ammonia, and ethane, $\mathbb{Z}_{2} \times \mathbb{Z}_{2}, D_{6}$, and $D_{6}$ $\mathrm{x} \mathbb{Z}_{2}$ respectively, has multiple advantages. First, it exposes students to the kinds of group structures that compose the majority of chemically important symmetry groups; cyclic groups, dihedral groups, and the direct product of $\mathbb{Z}_{2}$ with cyclic and dihedral groups. Second, it gives students a number of opportunities to test out their method. After a few molecules, students seem to gravitate towards a particular method. 
Step 2: Describing and naming the symmetry group with an encapsulated group representation as a model-for the symmetry group of a molecule (Model 1)

As students investigate and describe the unique collection of symmetries for a variety of molecules, they will use various group representations to inscribe their collections of symmetries. These inscriptions and representations could include geometric figures and Cayley tables as seen in Figure 32 above respectively, group presentations, conventional group names, etc. and they are all an important component of the students' emergent model. Knowing what kinds of representations encapsulate a symmetry group is nontrivial for students. Students often produce a Cayley table in order to organize, test, and identify that they have indeed a found a group structure, however students will then continue on their search using their Cayley table to try and produce a more concise group representation. This is a powerful inclination because it helps to anticipate the need for the use of a "smaller" group representation. Eventually the students will have to decide on a way to describe the different groups of symmetries with a concise/encapsulated enough representation to be used as an output for their classification system. Therefore, it is important for students to have some kind of experience with naming groups in order to be successful in this step as it requires the students to produce some kind of description of a symmetry group.

If the students have any traditional group theory experience, they will likely be trying to describe the group structures by conventional names; $\mathbb{Z}_{4}, D_{6}, \mathbb{Z}, \mathbb{R}$, etc. The students' mathematical activity and amount of time spent arguing that the sets of symmetries of the molecular models, as identified by the students, are isomorphic to more conventional 
group names may vary greatly depending on the mathematical background of the student. For the students in TE3 their lack of previous exposure to group theory meant that conventional group names were not available. Instead the students in TE3 were supplied with additional tasks in order to help them create group names. Their mathematical activity is described below and the supplemental tasks are described in detail in Chapter 7: Paper 3: Productively Applying and Adapting a LIT for Different Kinds of Mathematical Preparation.

Whatever group names students end up using they serve as part of the students' emergent model for a strategy for describing a symmetry group (Model 1) that feed directly into their more general classification system (Model 2) by serving as the outputs to a flowchart. This manifestation of their model can be described as a chain of signification (Gravemeijer, 1999). A chain of signification is constructed when the activity with one sign comes to signify activity with a previous sign. A sign in this case is the students' encapsulation of the group concept using smaller and smaller representations until ultimately referring to groups by group names. The overarching chain of signification can be seen in Figure 33 below. The actual students' group representations can be seen by linking the students' activity described in Step 1 above and throughout Step 2 below. This student's use of group names is both the end of the chain of signification and also the final expression of their Model 1.

Group names
$\left.\begin{array}{l}\text { List of rules / Group presentation }=\text { signifier 2 } \\ \hline \text { Cayley table }\end{array}\right\}$ signifier 3
Geometric images $1=$ signified 2
Figure 33. Preliminary chain of signification for Model 1.

Sample Tasks and Prompts for Step 2: 
For students with previous group theory experience, deciding on names for various group structures is an activity based on establishing isomorphisms between their groups of symmetries and conventional group structures. For students with ample group theory experience, establishing isomorphisms is non-trivial and may involve short semantic conversations as observed in TE1 when Emmy and Felix considered groups of order four after having identified four symmetries for water, each their own inverse;

Emmy: I think I know that there is a cyclic group and the Klein IV group, I know that.

Felix: It's not cyclic (the symmetry group for water).

Emmy: It's not cyclic. And everyone is its own inverse in that group (Klein IV).

Felix: Same for this one too.

Emmy: Then it's Klein IV if everyone is order 2 except the identity.

The students in TE2, with introductory group theory knowledge, had some access to group names and therefore spent far more time and attention arguing that their sets of symmetries were isomorphic to their conventional representations, especially for ammonia and ethane. While the students in TE2 also seemed to recognize the structure of $\mathbb{Z}_{2} \times \mathbb{Z}_{2}$, the Klein IV group, fairly quickly in their Cayley table for the symmetries of water, they were less sure what group they had once the groups got larger. For a more indepth conversation on the students in TE2 and their activity around establishing 
isomorphisms and exploring direct products, see Chapter 7. Here I want to focus on a task sequence designed to help students develop group names who don't have access to conventional group names.

Recall that the students in TE3 began the teaching experiment without any previous expose to group theory and so I needed to prepare them so that they could engage with the LIT. In keeping with the underlying theoretical perspective that students should be given opportunities to reinvent mathematics, I chose to prepare the students by engaging them in a pre-existing LIT found in the IOAA curriculum for the guided reinvention of the group concept (Larsen, 2010a). The students were asked to explore the symmetries of a square and in doing so reinvented the group concept. One result of using IOAA is that students are taught to use group presentations as representations for groups. In TE3 we referred to each group presentation as their "list of rules" that one would need to recreate a list of elements and/or a Cayley table.

Sometimes these "list of rules" had strictly more necessary information than in a traditional group presentation which only lists orders of generators and relations. The extraneous information was often group axioms or relations illustrating the identity property of the trivial symmetry that had also been found to be true, and with each molecule the students listed less extraneous information as seen in Figure 34.

\begin{tabular}{|c|c|c|}
\hline Water $\cong \mathbb{Z}_{2} \times \mathbb{Z}_{2}$ & Ammonia $\cong D_{6}$ & Ethane $\cong D_{6} \times \mathbb{Z}_{2}$ \\
\hline $\begin{array}{ll}O F=F & \\
O R=R \quad \text { Associative } \\
2 R=O \quad F R=R F \\
2 F=O\end{array}$ & $\begin{array}{l}R^{3}=0 \quad F^{2}=0 \\
O R=R \quad O F=F \\
R F=F R^{3}\end{array}$ & $\begin{array}{rlrl}R F & =F R \\
T^{2}=0 & R^{3}=0 \quad F^{2}=0 \\
F R=R F & R T=T R^{2} \\
& T F=F T\end{array}$ \\
\hline
\end{tabular}

Figure 34. Students' group presentations for the symmetry group of water, ammonia, and ethane from TE3. 
While the students' group presentations did serve as accurate representations for each of the symmetry groups, they were not compact enough to serve as outputs for a classification system. The students in TE3 needed to come up with something like a group name. From their work with water, ammonia, and ethane they had seen a variety of group structures. I wanted to give them more experience with additional molecules so that they had a larger example space to pull from when created group names. The students in TE3 were then given a second, bigger collection of molecules and again asked to find the symmetry group of each. The molecules and the students' group presentations for each can be found in Figure 35 below.

Prompt: Find the symmetry group for each of the following:

\begin{tabular}{lllll}
\hline Tetra-aza copper II & $\begin{array}{c}\text { Hydrogen } \\
\text { peroxide }\end{array}$ & Boric acid & $\mathrm{BrF}_{5}$ & cyclobutene \\
\hline $\mathbb{Z}_{4}$ & $\mathbb{Z}_{2}$ & $\mathbb{Z}_{3} \times \mathbb{Z}_{2}$ & $\mathrm{D}_{8}$ & $\mathrm{D}_{8} \times \mathbb{Z}_{2}$ \\
\hline
\end{tabular}

Student's "list of rules" i.e. group presentation for each symmetry group:

\begin{tabular}{|c|c|c|c|c|}
\hline $\begin{array}{l}T A C \\
R^{4}=0 \quad \text { u symmetries } \\
\text { No } F_{S} \text { No } T_{S}\end{array}$ & $\begin{array}{c}\text { Hydrogen Peroxide } \\
\qquad R^{2}=0\end{array}$ & $\begin{array}{l}\text { Boric Acid } \\
R^{3}=0 \quad T^{2}=0 \\
6 \text { symmutries } \\
T R=R T\end{array}$ & $\begin{array}{l}\text { Brain Slug } \\
4 \text { rotations } \\
R^{4}=0 \quad F^{2}=0 \\
8 \text { symmetries } \\
R F=\$ F R^{3}\end{array}$ & $\begin{array}{l}\text { Cyclobutane } \\
R^{4}=0 \quad F^{2}=0 \quad T^{2}=0 \\
16 \text { symmetries } \\
R F=F R^{3} \\
T R=R T \\
T F=F T\end{array}$ \\
\hline
\end{tabular}

Figure 35. Additional molecules given to students in TE3 along with the group presentations created to describe each.

Once the students produced accurate group presentations for each of the new molecules, they were ready to come up group names based on similar group structures. In order to do this, students were asked to create "score cards" for each of the molecules 
they had investigated they group the cards based on similar group types, found in Figure 36.

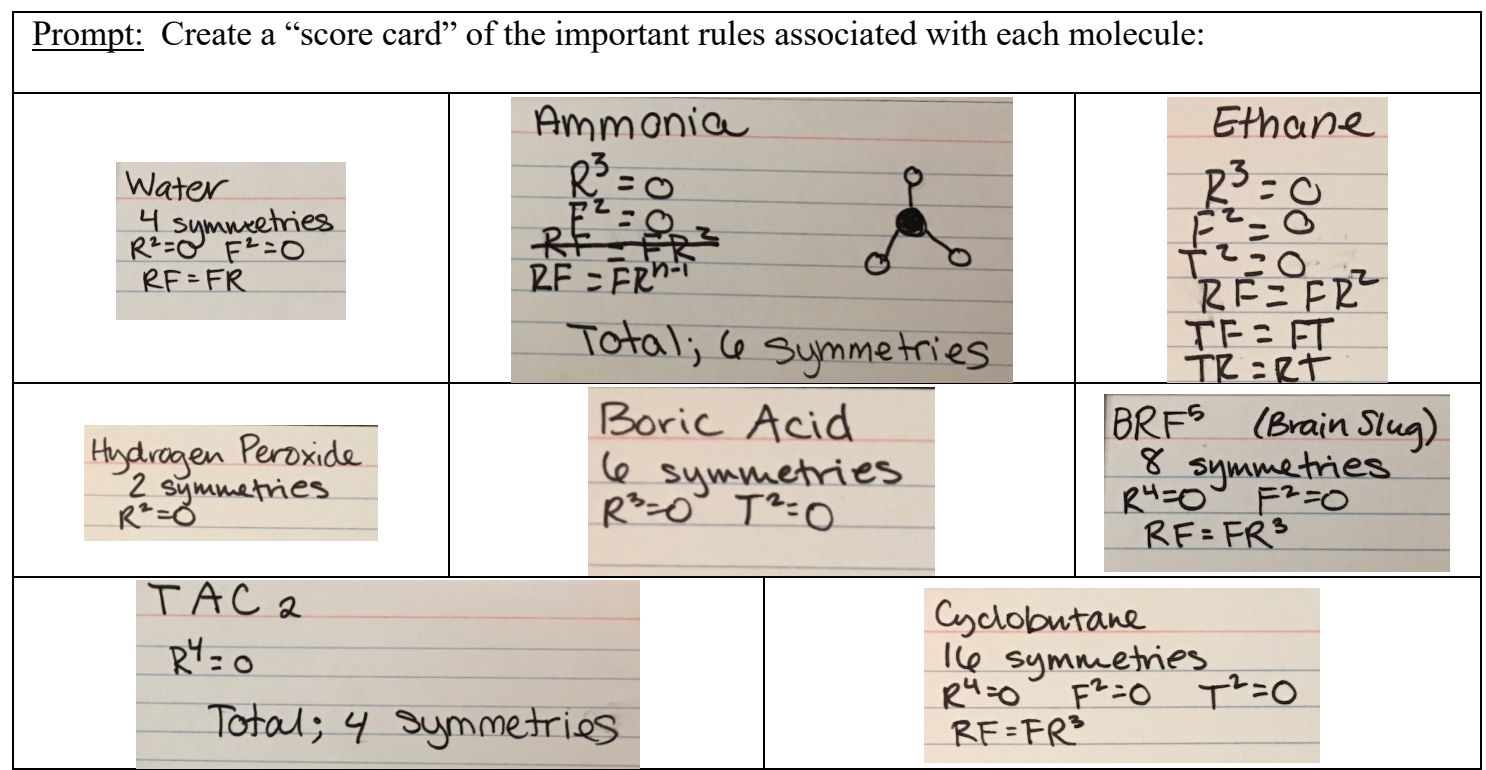

Figure 36. Student's "Score Cards" containing a group presentation for each molecule they have investigated, TE3.

When asked to group the score cards to determine types of groups, the students offered two different ideas, to either group molecules who had common symmetries, or to group them by the order of their rotation. Together they decide to group them by the order of rotation group, thus giving them three kinds of groups. I quickly remind them that with that strategy there will be 8 groups total because of the restriction on order of rotational groups due to chemical constraints which we had previously discussed. Then we had the following exchange:

Annie: If we go back to your instructions the fact that you wrote "if F" "if T exists" it makes me feel like it's important whether or not you know whether or not it has one of these kinds of symmetries or another.

Sophie: Yeah. 
Ada: Yeah.

Annie: Right, once I know the order of rotation, I know what to do with it (indicating to their instructions for determining a group). But really if something exists or not, to me that holds a little more weight.

Sophie: Yeah, that does, that's kinds of important. Not all of them have T's.

[They quickly rearrange the score cards into groups of groups that share the same symmetries.]

Annie: How does that feel?

Ada: Visually I think that they look different, like just oh gee what do these two have in common, (motions to Tetra-aza Copper II and hydrogen peroxide), versus oh gee what do these two have in common (motions to water and hydrogen peroxide), it's harder to picture, but it also, I feel like it means you're gaining more when you're dividing it into a group because you're grouping things together in a different way some way that you wouldn't just snap decision and look at.

Annie: It's not quite as superficial is it?

Ada: Yeah

Sophie: Yeah

Ada: You probably gain more information with that (motions to new grouping). 
The students second grouping can be seen in Figure 37 below, again grouped together based on existence of specific kinds of symmetries.

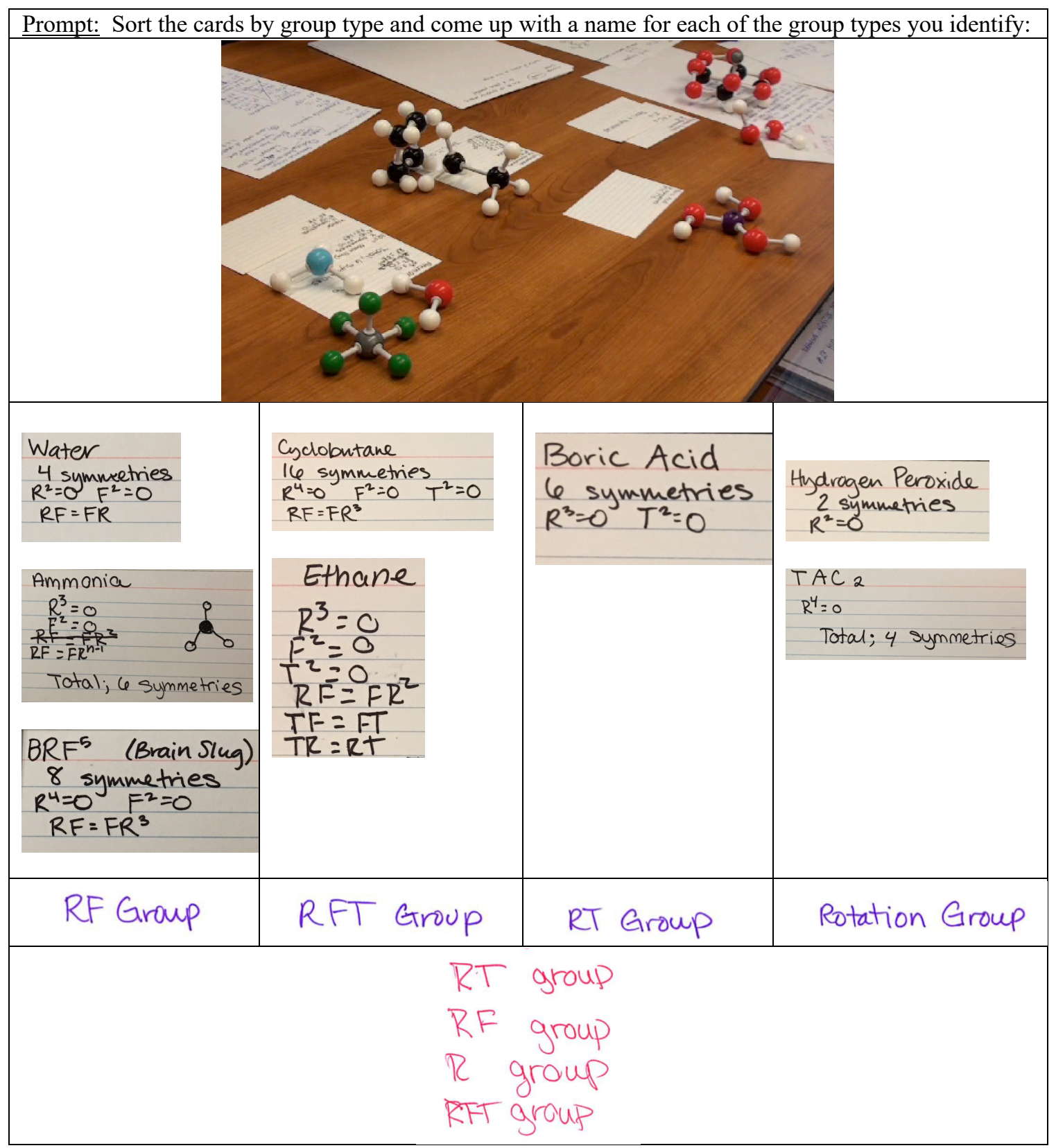

Figure 37. Students sorting of their score cards into four group types along with the names assigned to each type of symmetry group, TE3.

Step 3: Formulating an explicit system for determining the symmetry group of a molecule

(Model 2) as mathematizing the previous activity 
Once students have successfully described the symmetry groups for each of the molecules in the previous steps they are ready to reflect on their activity and explicate their methods. As students reflect on their strategy for determining symmetry groups, they tend to produce some kind of flowchart, decision tree, or list of questions to help identify the existence of various symmetries. While students are describing the various symmetries they identified, they are encouraged to also produce an accompanying instruction manual describing how to use their flowchart. These instructional manuals often contain the students' own definitions for various symmetries. While students may have tried a variety of methods for determining symmetry groups at this point by working with multiple molecules with varying group structures, they should have enough experience in order to articulate a method that describes a single streamlined activity. The final product of Step 3 may be inaccurate and/or incomplete, see Chapter 5: Paper 1: Developing an Active Approach to Chemistry Based Group Theoryor Bergman \& French, 2019 for detailed examples from TE1. The students should be pushed to create a system that can at least accurately identify the symmetry groups the students have already worked with which will be enough to begin testing and refinement. Below is the prompt used in TE3 to elicit the first written version of the students' system (Model 2) and the corresponding mathematical activity of the students.

Sample Tasks and Prompts for Step 3:

Prompt: What we want to do next, is to develop and describe a system for finding the symmetry group of any given molecule based on what you did with these three (water, ammonia, and ethane). This happens all the time in chemistry class, a student is given a molecule and they want to know what its symmetry group is because to a chemist its symmetry group gives vast insight into its properties. So assuming the end user knows what symmetries are and how to identify them, can we come up with a system that tells them what kind of symmetry group the molecule would have?

"if you are holding a thing (molecule), what information do you have to get from this (the model of the molecule) to get to this (the symmetry group)?" 
Figure 38. Prompt given to students in TE3 to promote the explication of their system for determining a symmetry group.

Students in TE3 were provided this prompt after having identified the symmetry groups of water, ammonia, and ethane. Recall, these students provided group presentations for each of the molecules as their group representation, as seen in Figure 38 above. So when asked to create a system for determining groups, for Ada and Sophie this meant a way to create group presentations as seen in the exchange:

Annie: So we want to do is come up with a system for say chemistry people or somebody else, who doesn't want to do all the group theory in the middle, they just want to go from; I have a molecule, through some kind of algorithm or flow-chart, to here's its group.

Ada: $\quad$ So their end group... they would desire...

Annie: So what was the most useful thing for you guys to know the whole group? What is the most sort of compact information that we can give them so that they know the whole group?

Ada: The rules!

Sophie: Yeah, the rules.

Ada and Sophie turn their focus on the rules to try and decide what the different parts of the rules are in order to help "the end user" create such a list. Ada starts by recreating each of their group presentations for each molecule water, ammonia, and ethane. 
Meanwhile, Sophie starts looking for commonalities between the group presentations using a Venn diagram seen in Figure 39 below.

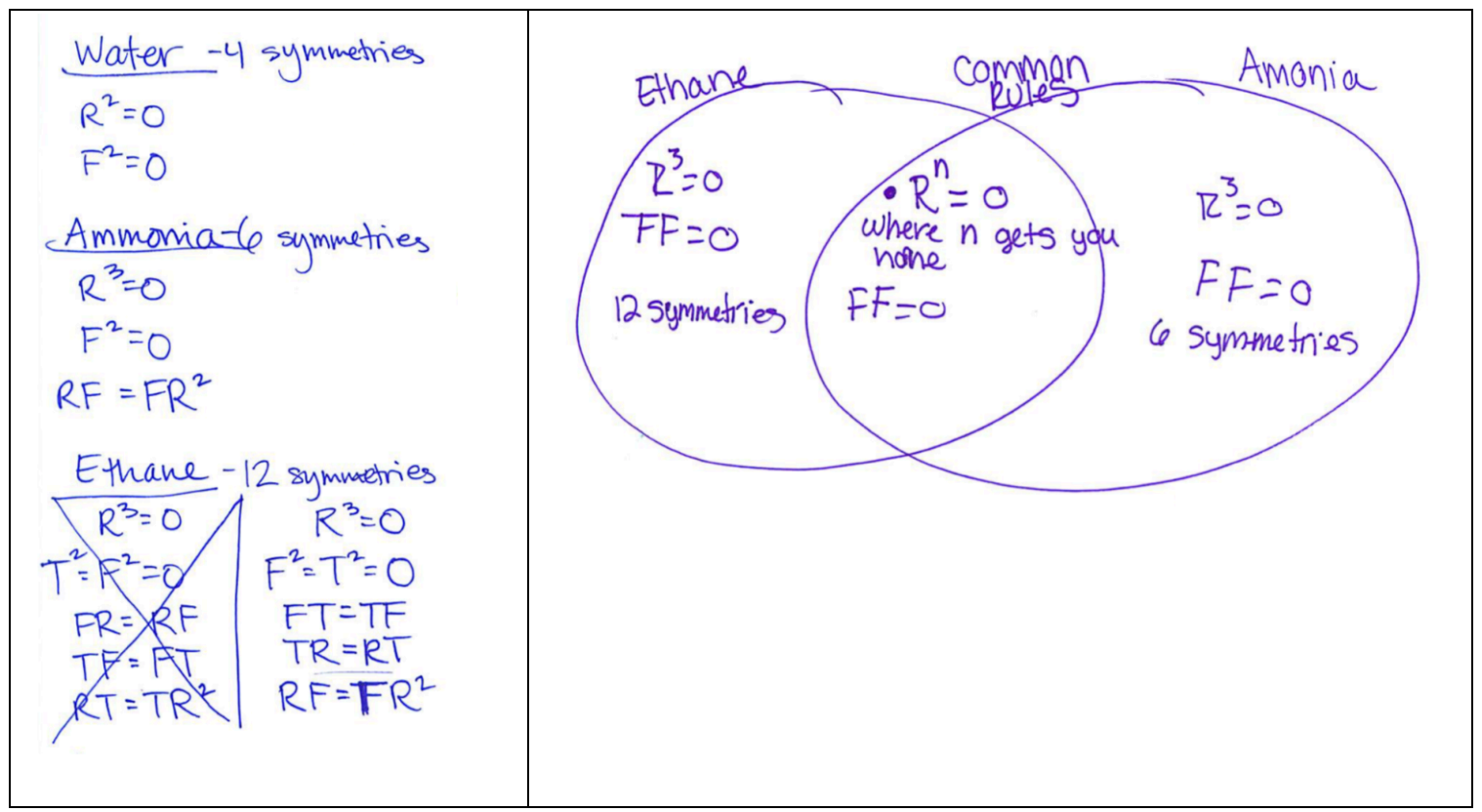

Figure 39. Student's work associated with trying to find commonalities between group structures, TE3.

After considering similarities and differences between the presentations, Ada eventually posits:

Ada: It would be nice if they started out by knowing what order of rotation.

Sophie: Yeah

Ada: ...cause that's like the main difference I'm seeing here.

Sophie: So maybe like if they can decide how many orders of rotation it has then they can find they find one component to the symmetries?

Sophie: Yeah. 
Ada: So maybe we could start out by asking a series of questions to be like, well how many rotations are in it, and then um, can we assume all of them are in 3-d?

Annie: Yeah, oh yeah, all molecules live in 3-d.

Ada: That's much easier to know.

From there Ada and Sophie start making a list of steps for someone else to start to identify a specific symmetry group, seen in Figure 40 below. While discussing their findings of how various symmetries interacted Sophie began to suspect that there was a relationship between a pure rotation $R$ and a reflection containing the rotational axis. This relationship can be referred to as the dihedral relation, as it is a fundamental relationship of dihedral groups. Her work can be seen at the bottom of her first attempt at her algorithm in Figure 40 below.

\begin{tabular}{|c|c|}
\hline 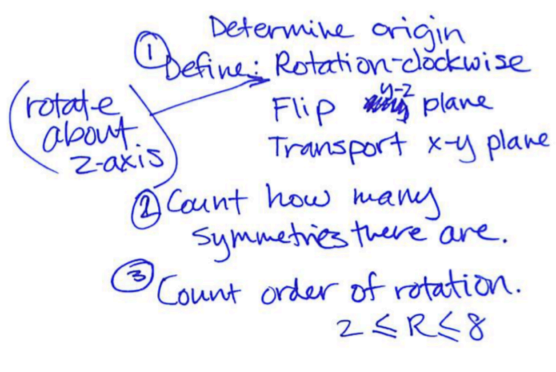 & 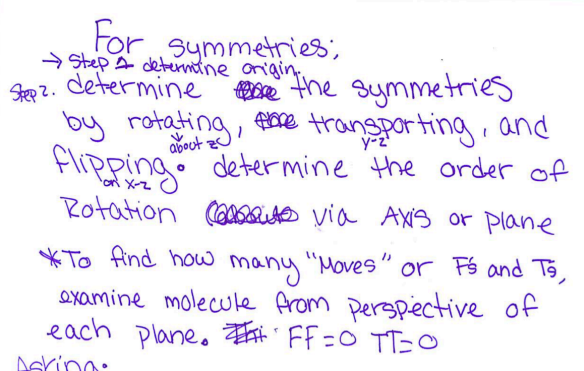 \\
\hline $\begin{array}{l}\text { If } R \& F \text { exist (in symuctios) } \\
\text { where } n=\text { order of rotation } \\
R F=F R^{n-1} \\
T \text { is communative with } \\
\quad R \& F \text {. }\end{array}$ & 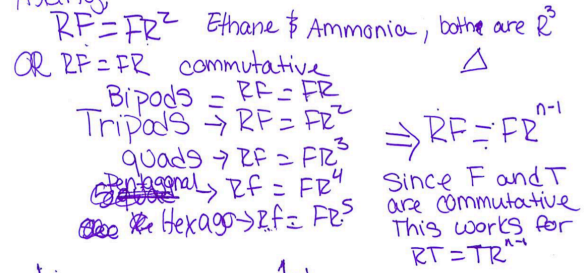 \\
\hline & $\because R$ \\
\hline
\end{tabular}

Figure 40. Ada and Sophie's initial attempts at explicating a system for determining a symmetry group, TE3.

Ada articulates their findings by saying: 
Ada: So the equation, that thing that we end up seeing in this pattern is that the motion of $R F$ is gonna equal $R F$ to the $n-1$, if $n$ equals the order of rotation. That might be true for everything, which is awesome, but I don't think it helps narrow down.

Annie: Was it... well I mean it's not true if you don't have $F$ and $R$.

Ada: That's true.

Ada: $\quad$ So it's only true if $R$ exists and $F$ exists.

Sophie: Ok, and we're completely pretending that $T$ is not a thing right now.

Annie: Right now it seems like we're just talking about the relationship between $F$ and $R$.

Sophie: Yeah, ok this is just for the, oh yeah. That's kind of cool, that did a thing. I wonder what happens if we talk about $T$ and $F$ though? They're commutative so $F T$, what about $T$ and $R$ they're commutative too, so it's really just these two that are the important ones cause they change things.

This prompts Ada to write out the rules they have determined so far (see Figure 40), and then asks:

Ada: Is it useful if we just tell them that rule?

Annie: Seem like it.

Ada: Cause then they just know. 
The inclination to begin the algorithm with counting rotations, then checking for reflections, followed by identifying a group based on the interaction between these symmetries was not isolated to TE3. In fact in each of the teaching experiments, students explicated similar strategies, as seen in Figure 42 below.

\begin{tabular}{|c|c|}
\hline TE1 & TE2 \\
\hline 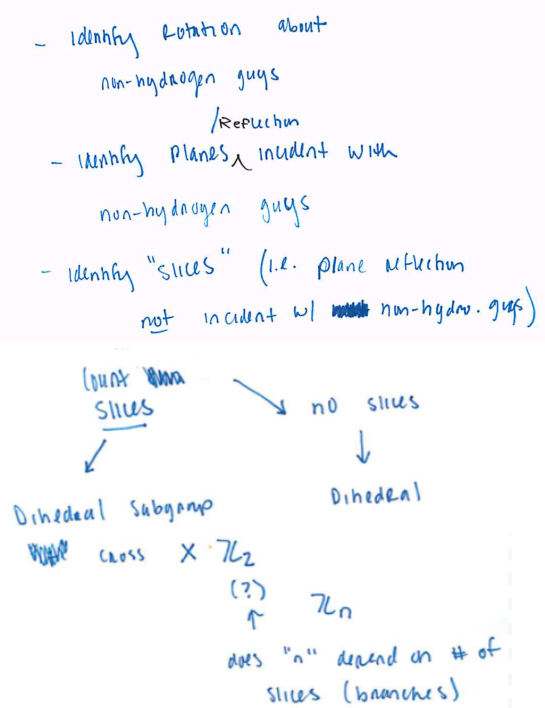 & $\begin{array}{l}\text { 1. Stare until to find reflections and rotations. } \\
\text { 2. Map out the symmetries in a useful way. Find prssible } \\
\text { unique end states which are indiscernible from or. The } \\
\text { brigineal state, from any given starting perspective. } \\
\text { 1. Look at "Projections" of ubjects for obvious symmetries } \\
\hat{v} \text { Test symmetries for unique results } \\
\text { Explore possible unique configurations (Independent of } \\
\text { the transformations that generate them). }\end{array}$ \\
\hline
\end{tabular}

Figure 42. Arthur and Stu's initial model for a system for determining symmetry groups in general, TE2.

Each of these articulations serves as the first explicit instantiation of the students' model of how to identify a specific symmetry group that will eventually be tested and refined as it feeds into their system for classifying symmetry groups (Model 2).

Step 4: “Applying” the system for determining the symmetry group of a specific molecule (Model 2) in similar situations

Students need an opportunity to test and refine their model in contexts that are structurally similar to what they have already seen, as it allows them gain more of a sense of ownership over their model students. This also gives students an opportunity to test 
the usefulness and applicability of their classification system. As students test and refine their model it begins to transition from a model of the students' own informal activity of identifying the symmetry group of a specific molecule to a model for classifying symmetry groups in general (Model 2). Each refinement of the students' model is further evidence of this transition.

Once the students have completed Step 3 by constructing a method for identifying symmetries for molecules similar to what they have already experienced, they are ready for the first round of testing and refinement. For students with lots of group theory experience, much of this testing and refinement can be done through thought experiments considering the existence of various combinations of reflections and rotations. See Bergman \& French, 2019 or Chapter 5 for more detail on how students in TE1 adapted their flowchart through a series of though experiments. Below is the story of TE3 and how they began to test and refine their system.

\section{Sample Tasks and Prompts for Step 4:}

Recall that Ada and Sophie's engagement with the LIT did not happen in exactly the order as presented here. After Ada and Sophie were equipped with a strategy for identifying symmetry groups (seen in Figure 41 above) they were asked to find the symmetry group for each molecules found in Figure 43 below. Notice that these are the same molecules as are referenced in Step 2. For Ada and Sophie, their description of a strategy (Step 3) came before their use of compact group representations (Step 2); and in order to create compact group representations they were given molecules containing familiar symmetries (Step 4) as seen Figure 43 below. 
Prompt: Find the symmetry group for each of the following:

\begin{tabular}{|c|c|c|c|c|}
\hline Tetra-aza copper II & $\begin{array}{l}\text { Hydrogen } \\
\text { peroxide }\end{array}$ & Boric acid & $\mathrm{BrF}_{5}$ & cyclobutene \\
\hline $\mathbb{Z}_{4}$ & $\mathbb{Z}_{2}$ & $\mathbb{Z}_{3} \times \mathbb{Z}_{2}$ & $\mathrm{D}_{8}$ & $\mathrm{D}_{8} \mathrm{x} \mathbb{Z}_{2}$ \\
\hline
\end{tabular}

Figure 43. Additional molecules given to students to test their system for identifying symmetry groups in TE3.

The five molecules in Figure 43 gave students an opportunity to both test their system and work with the most common group structures found in chemically important symmetry groups cyclic, dihedral, cyclic $x \mathbb{Z}_{2}$, and dihedral $x \mathbb{Z}_{2}$. As described in Step 2 above, Ada and Sophie successfully produced group presentations for each of the molecules using their system (see Figure 35 above). The elaboration of Step 2 also describes how this activity fed into the creation of group names $R, R F, R T, R F T$.

Prompt: Using your new group names $R, R F, R T$, and $R F T$ flesh out your directions for findings a symmetry group so that it is more like a decision tree that spits out the right group name.

Figure 44. Prompt given to Ada and Sophie during Step 4 of TE3

Ada and Sophie were then asked to use these new group names to refine their system from a sequence of questions into some kind of flowchart, decision tree, or algorithm that spits out that correct group name. This led them to create the flow-chart found Figure 45 below. 


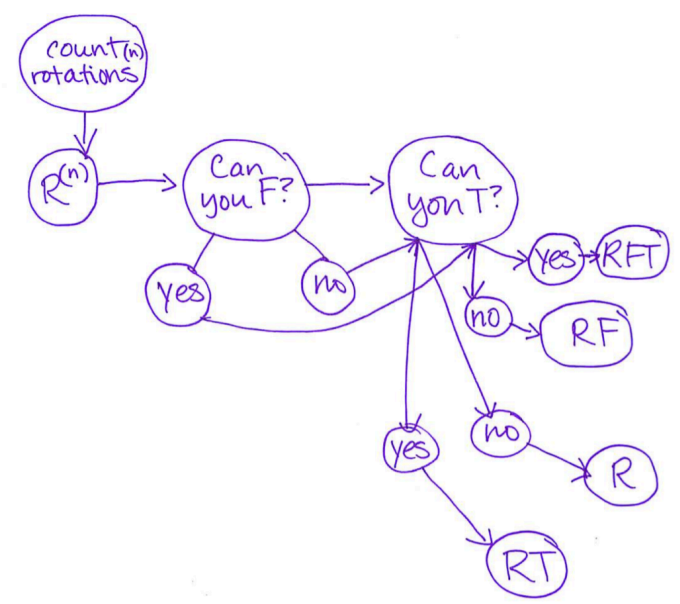

Figure 45. Ada and Sophie's model for identifying the symmetry group of a given molecule incorporating their newly created group names, TE3.

The specific molecules used in this prompt were chosen because they have groups composed of the same symmetry elements that students have already seen. By considering new molecules with very similar group structures to the ones they have already investigated, say $\mathrm{D}_{8} \times \mathbb{Z}_{2}$ instead of $\mathrm{D}_{6} \times \mathbb{Z}_{2}$, students can test their model which serves two purposes; 1) it allows them to see if their model is general enough to handle things that are slightly different than what is was built from and 2) it allows them to see if their description of the method is well enough articulated to serve as guidance to apply it to new things. By considering new group structures, such as a pure cyclic group, students can also refine their model to include more kinds of groups. By the time students complete Step 4 they should be quite confident that their model will produce the correct symmetry for any molecule that has a group structure composed of rotations and/or reflections, with no more than one rotational subgroup order three or higher.

Step 5: "Applying" the system for determining the symmetry group of a specific molecule (Model 2) in different contexts 
As students test and refine their model it continues its transition from a model of the students' own informal activity to a model for more formal reasoning, and again each refinement of the students' model is further evidence of the transition of their emergent model. During the first four steps of the LIT, the students are likely to be unaware of the existence of inversion symmetries. This means that their method for classifying groups could produce incorrect results. Step 5 of the LIT is designed to support the students in generalizing their approach to include this final primitive symmetry.

Sample Tasks and Prompts for Step 5:

Ethane is an excellent molecule to continue to test students' models to push them to refine them so that it can accommodate every type of three-dimensional symmetry. Ethane can present itself in two highly symmetric configurations, eclipsed or staggered, as seen in Figure 46.

Ethane in an eclipsed
configuration $\quad \begin{gathered}\text { Ethane in a staggered } \\ \text { configuration }\end{gathered}$

Figure 46. A description of ethane in either the eclipsed or staggered configurations.

Ethane has the same symmetry group up to isomorphism in either the eclipsed or staggered configuration, $D_{6} \times \mathbb{Z}_{2}$, however the collection of symmetries differs and therefore chemists refer to their symmetry groups as $D_{3 h}$ and $D_{3 d}$ respectively. Whereas ethane in the eclipsed configuration can be described as a collection of reflections and 
rotations, in the staggered configuration ethane contains an inversion symmetry not available when eclipsed.

Prompt: Find the symmetry group of ethane in a staggered configuration:

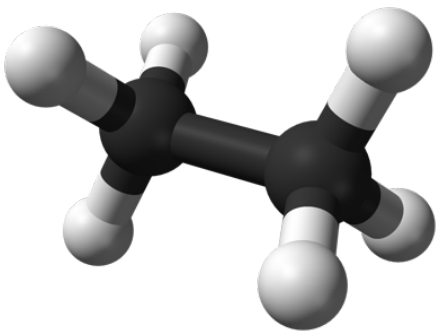

Figure 47. Prompt given to students in TE1, TE2, and TE3.

In TE3 when given ethane (staggered) the students struggled to identify the symmetries, especially the order 3 rotation. This was slightly different from the students in TE1 and TE2 who quickly gravitated toward rotations in each of the molecules. Instead Ada and Sophie made quick use of their newly developed flowchart from the previous step to determine that the molecule had no symmetries:

Annie: So wait, this guy has no rotations?

Ada: He's in that group, (they are both pointing to the $R^{n}$ output on their flowchart).

Annie: Ok, wait, she's (Sophie) saying $R^{0}$ and you're pointing to $R^{n}$.

Sophie: Where $n$ is zero.

Ada: $n$ can be zero

Annie: So this molecule has no symmetries?

Sophie: Not that we know of yet. So far, that's what we have so far. 
I told them to keep playing with the molecule and quickly thereafter Ada identified an order two rotation orthogonal to the principal axis along the center bond. She explained her finding to Sophie and while describing the result of the rotation, also noticed the existence of a reflection plane along the center bond incident with an opposing pair of hydrogen atoms.

Ada: So I'm thinking 4 symmetries we're up to now.

Sophie: Let's write them down. Ok so...

Ada: Alright so essentially we've got...

Sophie: You said $R^{2}$, and I could see the two 180 degree ones. ok.

Ada: And then if we agree on the orientation there's either an $F$ or a $T$. So which plane you want where?

Ada and Sophie's system for finding a symmetry group always included descriptions of the symmetries using orientation of the molecule, and so they used that to identify what kind of reflection they have found. After anchoring their newfound rotation along the z-axis (see their directions for orienting in Figure 41 above), Ada offered the group $R^{2} T$.

While Sophie was trying to visualize the 180-degree rotation discovered by Ada, she 'incorrectly' performed a 120-degree rotation along the principal axis. I decide to remind them of this motion Sophie had performed and ask them if the motion is a symmetry using their definition of a non-deforming action that returns a molecule to its starting orientation, and they agree it does. Returning to their molecule they reorient it this time 
with the order 3 rotational axis aligned with the $z$-axis. Using their flowchart once more and this time with an order 3 rotation and a reflection plane containing the order 3 axis, they decide on the symmetry group $R^{3} F$ instead. At this point we have the following exchange:

Annie: Ok so where are we sitting with this guy right now? What (group) do we feel like he has?

Sophie: Um like 12 symmetries, but I don't know if that's right. I'm just guessing. Ada: $\quad \mathrm{Um}$, so 6 is where I'm landing because I have $R^{3}=0$ and $F^{2}=0$ and so that means that I have to have 6 symmetries.

Sophie: Ok, and no $T \mathrm{~s}$.

Annie: So, you have, $R^{3} F$. Ok so $R$ rotates him this way (I rotate the molecule along the center bond) and $F$ reflects him this way (I use my hand to indicate the plane of reflection also along the center bond). And $T$ was the one that kind of went front to back, or triangle 1 to triangle 2 .

Sophie: Umm hmm.

Annie: But you guys said that this (the 180-degree rotation orthogonal to the center bond) was a symmetry...

Sophie: yeah...

Annie: Is that right?

Sophie: Umm hmm (Ada nods).

Annie: If I only have combinations of $R$ 's and $F$ s, how am I ever going to achieve that (the 180-degree rotation)? 
Sophie: ...you have to have a $T$

Annie: But he doesn't look like he has a $T$.

Sophie: He doesn't look like he does. but...

Ada: $\quad$ Well the $T$ wouldn't work because $T$ is a plane that you are smooshing across and if you do that you end up with the opposite thing on the sides.

Annie: But we agree that this (the 180-degree rotation) is a thing.

Ada: That would be rotation about an axis, a different axis then the first one.

While it is totally mathematically possible to describe the group of symmetries for ethane using the three symmetries Ada and Sophie had already identified as generating elements this would have fundamentally changed their system (Model 2). At this point I could have let them add the option of a second rotation to their flow chart to see where that would have gone. In fact, the consideration of a second rotational axis is standard in conventional classification systems within chemistry as seen in Figure 21. However, because my goal for introducing ethane was also to introduce the inversion center, the last mathematically primitive symmetry in 3-dimensions, I chose instead to point out the need for a new kind of symmetry.

Annie: So there's something going on here so it's almost like I wish I had a $T$, right?

Sophie: Yeah 
Annie: A way to get front to back or one triangle to the other, so that maybe I can achieve that (180-degree rotation) without having to think about that as a rotation.

I describe the inversion center, and its effect on molecules to Ada and Sophie using their findings from ethane in the staggered configuration in the following exchange:

Annie: So right now in three-dimensional space, we have a symmetry that interacts with a plane and we have a symmetry that interacts with a line.

Ada: $\quad$ Um hmm.

Annie: Right? What do you think about maybe a symmetry that interacts with a point?

Sophie: I mean, I think it's definitely possible, I was curious of what you were going to say came after a plane, so I'm glad you said point.

Annie: So when I rotate him (ethane by 180 degrees orthogonal to the order 3 rotation along the main bond) this hydrogen goes to here.

Both: Yeah.

Annie: What if instead rotating it I give you a point in the very center, right, your center of mass. Where you invert through the point. So he's gonna go to there, he's gonna go up and move there, and he's gonna go up and over to that one. [all of this is accompanies by hand movements demonstrating the result of an inversion]

Sophie: I can see that, yeah. 
Annie: It's like a bloop.

Ada: Yep.

Annie: What do you think of that as a symmetry?

Sophie: Well that's...I...that's what I was feelin like in my head, but I didn't know if it would be legal because it's, it's not quite as oriented.

Annie: Well it's not and $F$ or a $T$ right, it's something new.

Sophie: Yeah, yeah. But you can feel that the symmetries are there because the shapes are the same on both sides so you know that they can do more.

Annie: That is kind of the amazing thing about symmetries right, is that there is a big gut reaction to a lot of this.

Sophie: Like you know, but it's how...

Annie: So let me introduce you guys to the inversion center, the last what we would call primitive symmetry in three-dimensional space.

Sophie suggests they refer to this new symmetry as $I$ for inversion, as Ada adds the new symmetry to their list of rules for ethane (Figure 48). Ada then decides that they need to figure out how this new symmetry interacts with $R$ and $F$.

Ada and Sophie go on to determine the generator relations between the symmetries (Figure 48), which they then use to write a group presentation for their newfound group $R^{3} F I$ (Figure 48). 


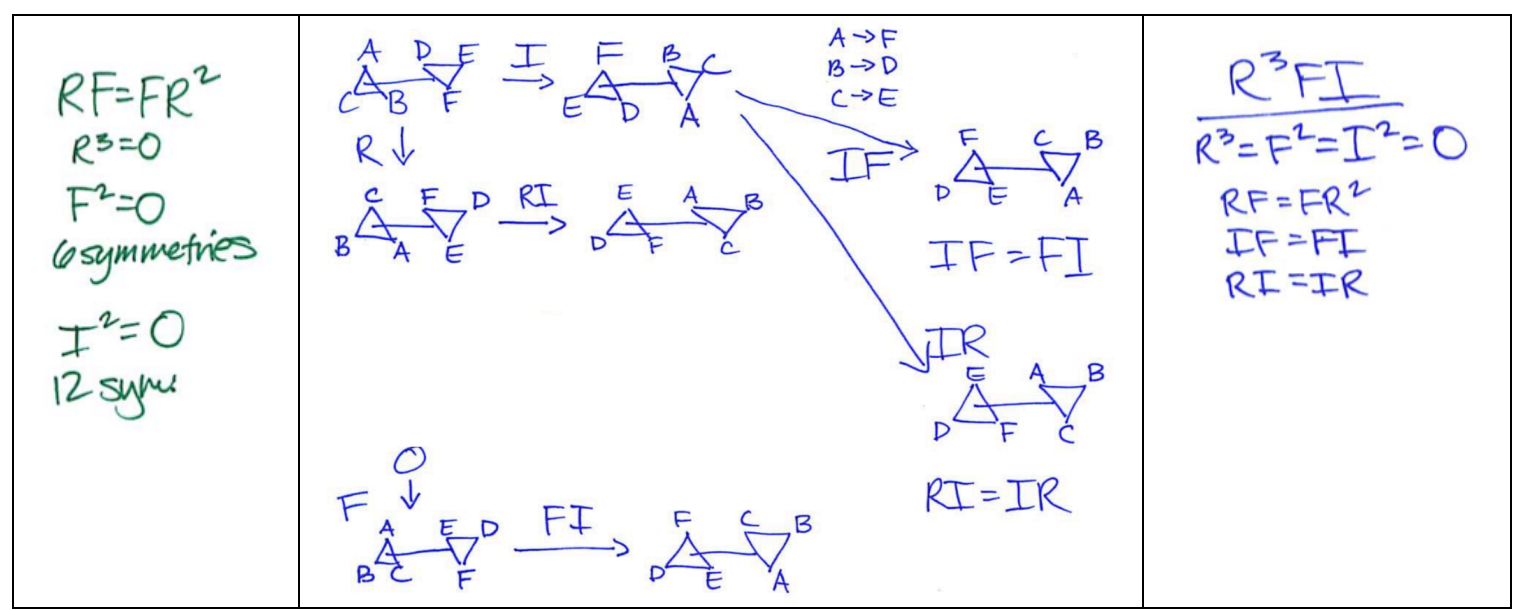

Figure 48. Student's written work while investigating an inversion in ethane.

Recall once more that Ada and Sophie didn't have prior exposure to conventional group theory concepts before the start of the teaching experiment and so the notion of isomorphisms, aka group equivalences was not available to them. Nevertheless, the students identified structural similarities between this group and the original ethane group. Sophie quickly recognized similarities between how a horizontal reflection $T$, and an inversion center $I$ interact with other kinds of symmetries.

Sophie: I's a lot like $T$.

Ada: Yeah.

Annie: What do you mean, $I$ is a lot like $T$ ?

Sophie: Well just as like the way the he's commutative...

Annie: So like how he participates in the group structure?

Sophie: yeah How he works with, his other friends in the group.

When I asked them how I would fit into their flowchart, Ada immediately responded, "I would just take an extra bubble and (write/ask...verb) 'Can you I?'”. While debating 
where in the flowchart the new question should fit, they also begin to wonder how the addition of $I$ will affect their outputs:

Ada: So when we draw this (the new flowchart), what do we want to have it lead to then?

Sophie: If it's invertible? Um, what's the order of invertibility?

Ada: 2?

Sophie: No, I don't know, no, don't write that. I feel like $I$ should be before or after you ask if it can flip (reflect about the vertical plane) or if it has to... can it do that without flipping?

Ada: In my brain I want to put it next to $T$ for some reason.

Sophie: Cause they're a lot of the same things...

Ada: They kind of feel the same.

Sophie: Yeah.

Ada: Ok, so are we re-naming these four groups and adding an I in there? That's the other thing are we filtering to different groups? Because if "Can you $I$ ?" ...well $I$ is not included in these four (groups), so I feel like we need a new flow chart with more groups right?

Ada posits that there will be four new groups with the addition of $I$, this giving eight different kinds of symmetry groups all together. She lists them out in Figure 49 below. 


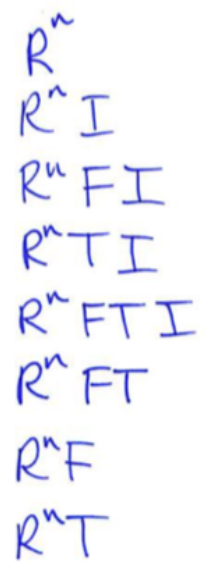

Figure 49. Ada's list of possible new groups considering the addition of the inversion center.

While Ada and Sophie recognized many similarities between the symmetries $I$ and $T$ without the concept of isomorphism they had no mechanism (or need) to collapse their set of groups. Ada and Sophie ultimately adapted their flowchart to include both a new question for the symmetry, $I$, and new kinds of symmetry groups, as seen in Figure 50 below. The result of their non-collapse is actually quite exciting as it produces a classification system similar to those used by chemists. Again, chemists are interested in both the symmetry group of a molecule, and also the symmetries themselves. As it is the elements of the symmetry group which give insight on where to expect certain observable properties such as modes of motion of a molecule. For example, one would expect a molecule with a single order two rotation to move quite differently than a molecule with just an inversion center; however to the mathematician they both have symmetry groups isomorphic to $\mathbb{Z}_{2}$. 


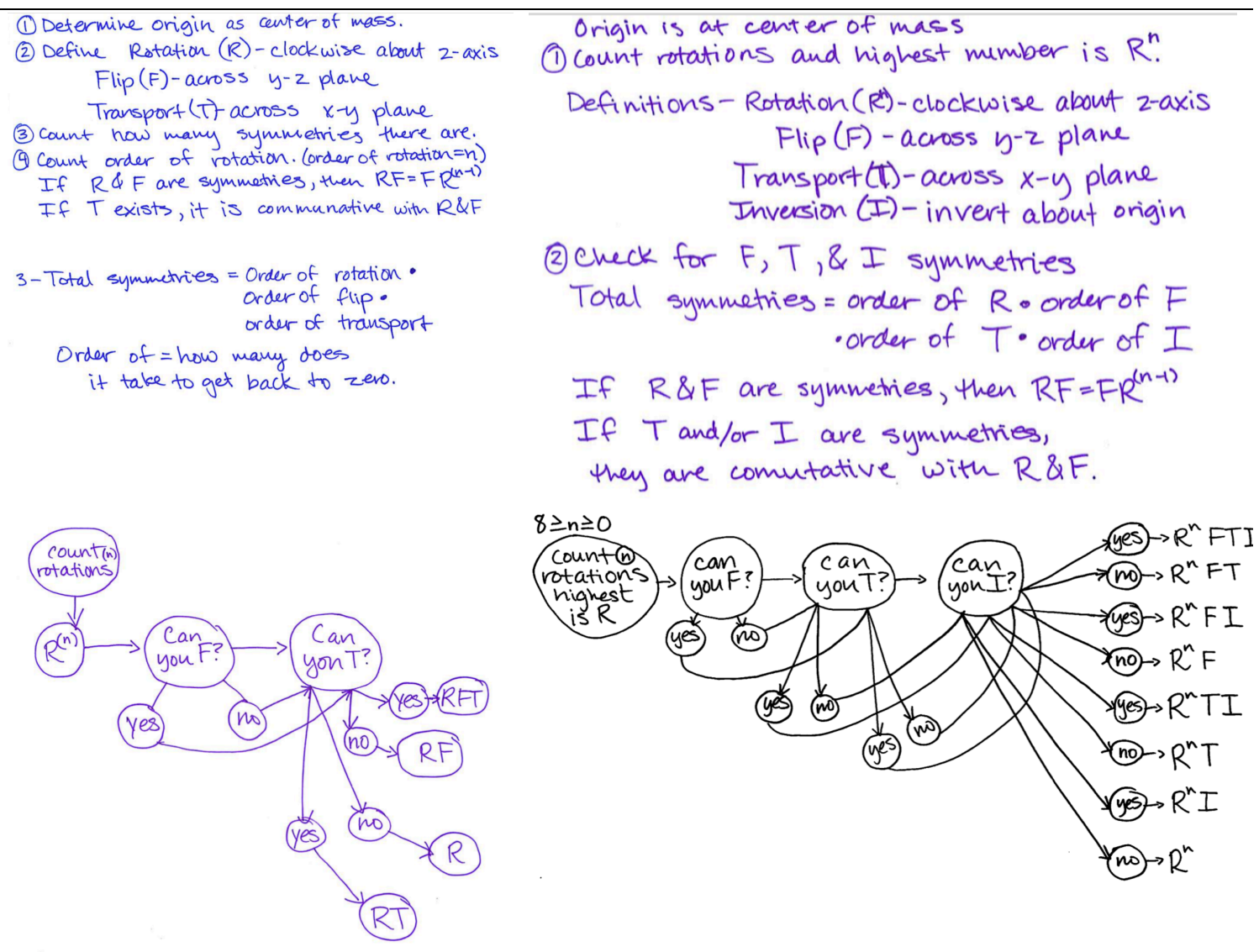

Figure 50. Students' systems for determining symmetry groups before (left) and after (right) considering an inversion center, TE3.

In TE1 and TE2 the discovery of the inversion center only led the students to adapt their flowcharts to include a new kind of symmetry, unlike Ada and Sophie they didn't include any new groups. This was not surprising as the students had a more functional understanding of isomorphism classes of groups. Since the new symmetry didn't produce a new kind of group structure, students made minor adjustments to their work. Both students in TE1 and TE2 included the consideration of the inversion center adjacent to their inquiry of a horizontal plane. Adjustments to the flowcharts in TE1 and TE2 before and after the consideration of an inversion center can be seen in Figure 51 below, in green and purple respectively. For a more detailed description of the mathematical 
activity of the students in TE1 can be found in Chapter 5: Paper 1: Developing an Active Approach to Chemistry Based Group Theory.

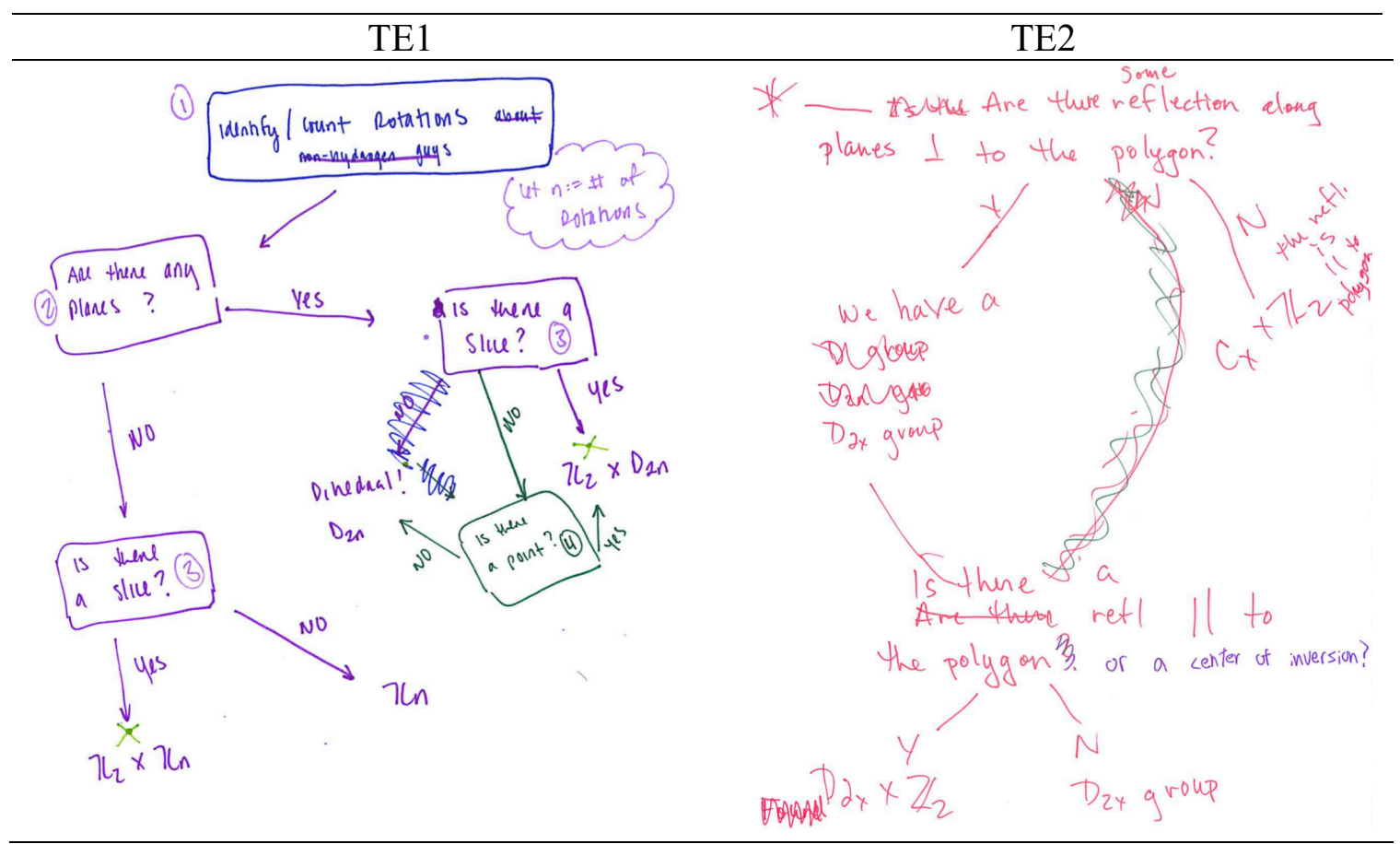

Figure 51. Students' systems for determining symmetry groups from TE1 (left) and TE2 (right) after considering an inversion center.

One last way student can test their model is on very symmetric molecules with high order symmetry groups. While these types of molecules have new symmetry groups than those the students have previously experienced, they are composed of the same symmetry elements the students have already used in their model. For a conventionally accurate classification system students should be given an opportunity to consider how to include very high order symmetry groups in their model. Highly symmetric molecules have symmetry groups that are quite different than any of the groups the students' model might predict. The molecules with 'special groups,' are those with more than one axis of order 3 rotations or higher, which result in symmetry groups of $\mathrm{A}_{4}, \mathrm{~A}_{4} \times \mathbb{Z}_{2}, \mathrm{~S}_{4}, \mathrm{~S}_{4} \times \mathbb{Z}_{2}, \mathrm{~A}_{5}$, and $A_{5} \times \mathbb{Z}_{2}$ and those with an infinite rotational subgroup, i.e. linear molecules. While 
the group structure might be at best unfamiliar and at worst unknown to the students depending on their prior group theory experience, they are all generated by the same three primitive symmetries the students have been working with all along, rotations, reflections, and an inversion center. Therefore it is possible students could produce presentations for these groups even if they could not identify the conventional representative of the isomorphism class. Unfortunately, due to time constraints this type of activity was never tested during the course of this study. A sample and prompt are provided in Appendix A along with the symmetry group of each molecule provided.

\section{Step 6: Justifying generality / robustness of the classification system (Model 2)}

After multiple rounds of testing and refinement students, should be ready to argue for the generality of their model and its ability to correctly identify the symmetry group of a given molecule. The level of formality, abstractness, and generality of the student's arguments may vary wildly depending on their own mathematical background.

Furthermore, the task of proving there can be no other three-dimensional symmetries than the types they have already considered is highly non-trivial and involves mathematical activity quite different than that involved in the first 5 steps of the LIT.

\section{Sample Tasks and Prompts for Step 6:}

\section{Prompt:}

1. Do you think your system captures every possible molecule?

2. How can you be sure that your system captures every possible molecule?

Figure 52. Sample prompt given during Step 6 of the LIT

The students in TE2 and TE3 they were only able to speak to what they had experienced, and desired interaction with more examples. Evidence of this was given by 
Stu when asked about the generality of their system (Model 2), Stu provided the following observation:

Annie: Do you think you, from what you've seen and what you've done. Do you think that you have gotten all the possible symmetry groups or molecules and if you don't what else do you think you'd want to know to argue that your flowchart is complete?

Stu: Well cubes are a big deal so are there, we've only talked about um $n$ rotations, one group of rotations that have an order. There could be more than one group of rotations, that's possible with more than one order. Um we could talk about, could we talk about reflections in 3-planes? We probably could. Yeah none of these have had that, but they certainly could.

Arthur: None of these have had what? Reflections in...

Stu: $\quad$ Yeah like if you think of $\mathbb{R}^{3}$.

Arthur: Umm hmm.

Stu: You could reflect along the z-axis, the x-axis, and the y-axis, for example and none of these have done that and a cube would handle both of those, would be in both of those categories, I think.

Annie: So maybe getting a handle on really high symmetry stuff?

Stu: $\quad$ Yeah.

Annie: You recognize that high symmetry things like cubes are still out there.

Stu: Yep. 
Arthur: Yeah.

Only the students in TE1 showed some understanding of what they would need to do to start answering the question. When asked if they could use their system to identify the symmetry group for any given molecule, they said the following:

Annie: And you think that you can identify the symmetry group of any...

Emmy: Give us a molecule Annie, just give it to us.

Annie: I don't have any more molecules, but hypothetically that means that you think you can identify the symmetry group of any molecule using rotations, planes, slices, and points. How do you know that those are all the kinds of symmetries that we have in 3-space? How would you start to think about this? I don't need a proof by the time you walk out today, but...

Emmy starts listing the motions they've identified so far.

Felix: What else is going to happen, let's think about this.... it has to keep the distance and the angle...

Felix starts using his hands to create a makeshift 3-dimensional coordinate system (seen in Figure 53) and mumbles inaudibly about different symmetries. Eventually I reiterate my question:

Annie: How would you know that there weren't any more. 
Felix: This is what's coming to my head, all the ways that I'm looking at like um vectors on the $x$ going out one, on the $y$ going out one, and on the $z$ going out one and how we can transform these, cause the angles have to remain the same and the length of the vectors have to remain the same. So how can we transform them through planes, we got the planes, through rotations, and the point switches everything upside down. Can we do something else to them? I feel like, no. I feel like no.

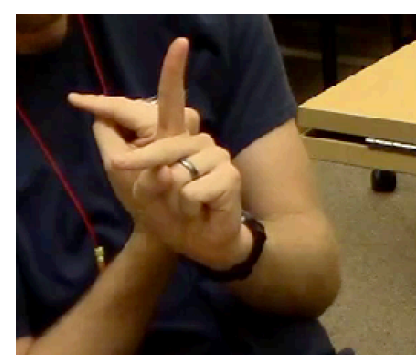

Figure 53. Felix's gesture of three-dimensional basis vectors, TE1.

Annie: So you're saying I can describe all my symmetries using some kind of basis elements in 3-space?

Felix: Yes.

Annie: Emmy do you think that's a reasonable approach?

Emmy: I guess so, if you want to do a bunch of linear algebra.

Witness: Can linear algebra offer a potential way out of this problem?

Emmy: What problem are we in?

Annie: Do you have all of the kinds of symmetries in 3-space?

Emmy: Oh prove it.

Witness: You're talking about basis elements being transformed, in my linear algebra brain that sounds like a linear transformation.

Emmy: Yeah. 
Witness: Which makes me wonder A. are symmetries linear transformations? and B. can that help us figure out if we have them all?

Felix: A, yes.

Emmy: Yeah, duh. A, yes. B, probably. Like $80 \%$ sure.

Witness: So how would you prove that a symmetry had to be a linear transformation?

In a short thought experiment about the various properties that symmetries would exhibit as linear transformations Felix posits that determinates would need to be 1 or -1 . Unfortunately we ran out of time before the students made any more progress and the teaching experiment ended.

\section{Conclusions}

In this paper I have presented a local instructional theory for the guided student reinvention of a classification system for chemically important symmetry groups that can be used for identifying the symmetry group of any given molecule. The LIT begins with an investigation with molecule with specific symmetry group properties which necessitates a student strategy for identifying symmetry groups. This strategy is leveraged into the creation of a classification algorithm for symmetry group identification. The LIT is described as the sequence of key steps identified as important milestones in the reinvention process. Each step is then described in further detail in terms of students' mathematical activity using the emergent model heuristic to describe how the step supports the evolution of the student's mathematical activity. For each step 
of the LIT I have also provided sample tasks and prompts that might evoke the particular mathematical activity necessary to complete the step along with empirical examples of student work.

The primary purpose for this study was to create an instructional sequence that gives students an opportunity to appreciate the applicability of and further their understanding of group theory by engaging in a real-world context. This theory of how to engage students in the reinvention of a classification system for chemically important symmetry groups is useful because it allows students to investigate important ideas in abstract algebra in a real-world context. Furthermore, by leveraging students' informal approaches in the creation of more formal mathematics, the students are given an opportunity to reinvent both fundamental group theory concepts such as group names, and something much more advanced, a classification system for symmetry groups.

While this research study successfully produced a LIT that is ready to inform a variety of instructional sequences for students with varying mathematical backgrounds, it also laid the groundwork for at least two directions for further research. The first direction is quite local and focuses on furthering the development and refinement of the LIT through additional teaching experiments. While the three teaching experiments reported here have produced ample evidence to support the LIT in a global sense, more evidence of students' mathematical activity for some of the later steps of the LIT would be particularly helpful in providing more detailed elaboration of these key steps. A follow-up study that extends the investigation to include very high order symmetry groups, e,g, the Platonic solids and focuses on how to support students in generating and structuring arguments for the completeness and generalizability of their classification 
algorithm would be a natural next step. The second way to build on this study would be to test the LIT with a new population of students, specifically chemistry students. Future studies might try to better understand, how might chemistry students engage in such an instructional theory? Or how might the mathematical activity of chemistry students be the same or different from that of mathematics students? Findings from a study conducted with chemistry students might lead to further adaptations or refinements of the LIT.

Lastly, there is work left to be done in order to scale up from working with a pair of students with unlimited time to a more typical classroom setting. Luckily, much of the process of designing and scaling up from a LIT to a classroom curriculum has already been laid out by Larsen, Johnson, and Bartlo (2013). Larsen and his team describe three overlapping stages of research which could easily be applied to my own work. First, they describe a series of small scale design experiments working with pairs of students aimed to develop the LIT; I would argue that this paper is evidence of my completion of this first stage. The second stage of research focuses on generalizing from the initial laboratory design context to an authentic classroom setting. This stage is important to understand more about how to time and pace the LIT as there are external factors on time and resources in classroom that simply are not present in a teaching experiment. Lastly, the third and final stage of scaling up a LIT from working with pairs of students to a full classroom is to generalize to instructors who were not involved in the design process. It is much easier for the lead researcher and curriculum designer to always be the lead instructor. It requires more explicit descriptions of the steps of the LIT and further 
elaboration of its rationale in order for someone else to teach it successfully where students are adequately supported in the reinvention process. 
Chapter 7: Paper 3: Productively Applying and Adapting a LIT for Different Kinds of Mathematical Preparation

Productively Applying and Adapting a LIT for Different Kinds of Mathematical Preparation

\section{Anna Marie Bergman}

A local instructional theory (LIT) describes a generalized roadmap for student reinvention of a particular mathematical concept (Gravemeijer, 1998). This path is a generalized sequence of steps, described in terms of student strategies and ways of thinking that have been identified as important milestones in the development of the fundamental ideas of the particular mathematical concept (Larsen \& Lockwood, 2013). The theory is local in the sense that it describes how the specific topic should be taught in order to support students in a guided reinvention process. However, the LIT differs from just a sequence of instructional tasks in the sense that the LIT focuses on the rationale behind choosing such a sequence of activities. This rationale provides an explanation of how the particular instructional activities comply with the intention to give students the opportunity to reinvent mathematics (Gravemeijer, 1998), but might leave the choice of specific instructional activities and prompts up to the instructor. In other words, instead of specifying particular tasks, the instructor (or adaptor) using the LIT is free to make specific instantiations using the rationale as a guide since the rationale explains the key features of the generalized tasks. This paper aims to help illuminate how a single LIT can inform two different instructional sequences to account for differences in student's a prior experiences.

Introduction 
Abstract algebra is a required course for almost every undergraduate mathematics student (Blair, Kirkman, \& Maxwell, 2013, p.54) and it is notoriously difficult for them (Larsen, 2010b; Leron, Hazzan, \& Zazkis, 1995; Weber \& Larsen, 2008). In fact much of the literature on student understanding in abstract algebra highlights student struggles (Weber \& Larsen, 2008). One of the many contributing factors to student difficulties in the subject is the abstract nature of the content of the course (Hazzan, 1999). In response, I recently conducted a design experiment aimed to develop an instructional sequence that presented concepts in abstract algebra in a way that was less abstract for students. In an attempt to begin reducing the level of abstract, I decided to use a real-world application of abstract algebra found in the context of inorganic chemistry.

In inorganic chemistry, where molecules are typically smaller and therefore higher more symmetric than the larger molecules studied in organic chemistry, a particular subset of symmetry groups are used to gain important chemical insights to molecular structures. Point groups (symmetry groups where at least the center of mass remains fixed) are often used to help understand experimentally observable physical attributes of molecules such as bond lengths, angles and modes of motion. Due to restrictions from both mathematics and chemistry, the number of chemically relevant symmetry groups (the collection of symmetries of some molecule under the operation of composition) is very well bounded. In fact, mathematically speaking there are only 32 chemically important symmetry groups up to isomorphism, (Zeldin, 1966). These groups are often classified and organized into a kind of flowchart, as seen in Figure 54 below, differentiated by the various symmetry transformations available to the molecule. 
Students are taught to use this flowchart in order to determine the specific symmetry group of a given molecule.

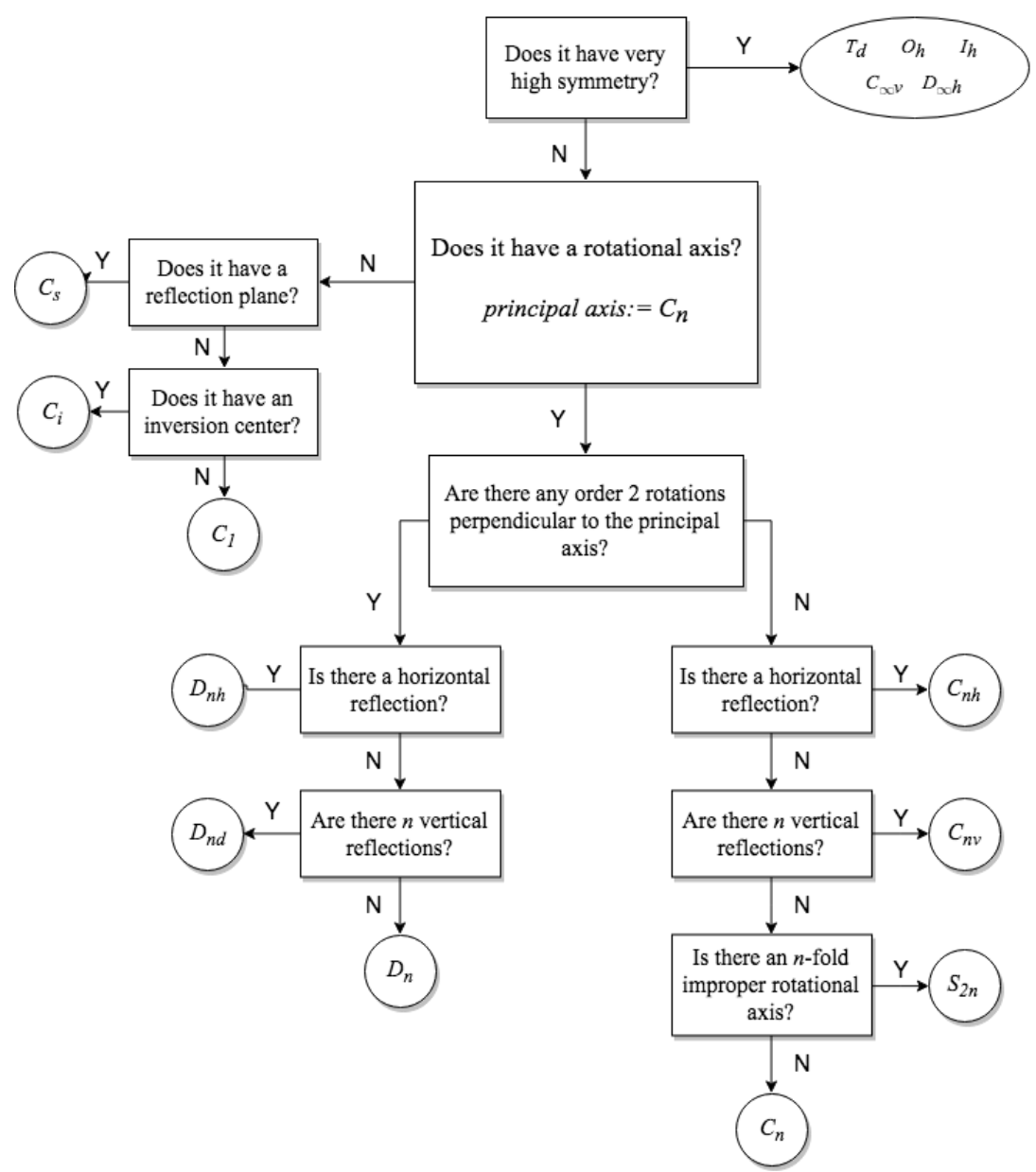

Figure 54. A typical flowchart given to undergraduates in inorganic chemistry.

For the design study reported on here, I decided to engage students in an investigation in abstract algebra by supporting them in reinventing such a classification system situated in the context of chemistry. It is important to note that since my goal was always to engage primarily mathematics students, the final flowcharts that my students developed were quite different from a standard flowchart found in a chemistry text in both subtle and important ways. These differences are explained in greater detail in Chapter 6: Manuscript 2. Figure 55 below shows one example of a flowchart created by the first 
pair of students in the design study. It important to notice that in both the standard chemistry classification system seen in Figure 54, and the students' classification scheme below, the outputs of the flowcharts are very concise representations of symmetry groups. In fact in both instances the group representation used can be described as a group name, arguably the most compact and encapsulated sign or representation of a group. Notice that symmetry groups have very different names in chemistry and in mathematics because (unfortunately) the languages of our disciplines are very different and trying to translate between the two is non-trivial for students and experts alike.

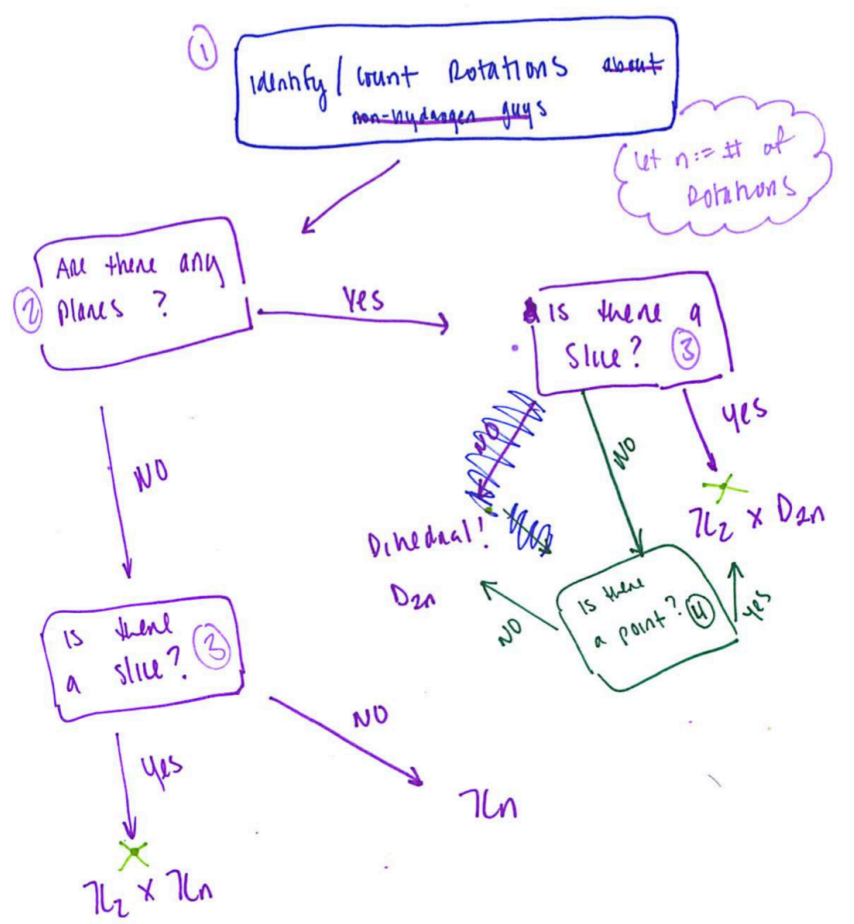

Figure 55. The student's final model for classifying chemically important symmetry groups from TE1.

\section{Theoretical Perspective}

The design study reported here utilized the instructional design theory of realistic mathematics education, RME, which provided both an underlying theoretical perspective and the accompanying design heuristics. The notion that mathematics is a human activity 
is the theoretical foundation that realistic mathematics education is built on and feeds directly into the reinvention principle, which is the first design heuristic of RME. The reinvention principle suggests that by engaging students in the guided reinvention of a particular mathematical concept, we are creating opportunities for them to meaningfully engage in mathematizing. When used as a design heuristic the reinvention principle promotes the idea of starting the reinvention process in a context that is experientially real to students (Gravemeijer \& Doorman, 1999). The reinvention/evolution from an informally situated student strategy to conventionally recognizable, formal mathematics is evoked using the second design heuristic of emergent models. Emergent models help to describe the overall evolution of the students' mathematical activity. Model in this sense is quite holistic as the model includes all the student inscriptions and ways of thinking necessary to slowly transition from informal to formal mathematics. The design heuristic of didactical phenomenology helps to inform the selection of contexts by promoting the use of tasks that can be solved with the very concept students are eventually intended to reinvent. For example, if students are intended to reinvent the concept of length asking students to organize objects of various length might promote the notion of organization by length. Each successive heuristic gives deeper insight on what the students should do in order to create increasingly formal and powerful mathematics. Since the formal mathematics that is eventually developed is rooted in the student approaches, this particular development process helps students have a greater sense of ownership over the formal mathematics created. The result of a design experiment using RME is a theory that describes how students might reinvent a particular mathematical concept. Such a theory is called a local instructional theory. 
There already exists quite a few LITs within the undergraduate mathematics education community, mostly focused on topics in algebra and differential equations. Some of the earliest LIT's created in undergraduate mathematics were in the domain of differential equations. Ongoing work has contributed to this body of work to now include the following topics: solving ODEs; numerical, analytic and graphical solution methods; solutions and spaces of solutions; linear systems; linearization; qualitative analysis of both ODEs and linear systems of ODEs; and structures of solution spaces. Together these LIT's and their associated instructional materials compromise a first full semester course in differential equations titled, Inquiry Oriented Differential Equations (IODE) (Rasmussen, Keene, Dunmyre, \& Fortune, 2018). Sequences of LIT's have also been developed in algebra, more specifically linear algebra and group theory. In linear algebra LIT's describing the reinvention of linear independence and span, matrices as linear transformation, and change of basis, diagonalization, and eigentheory, are used together in Inquiry Oriented Linear Algebra (IOLA) (Wawro, Zandieh, Rasmussen, \& Andrews-Larson, 2013). Lastly, the IOAA curriculum is a term-long inquiry-oriented group theory course built around three LIT's for the concepts of group, isomorphism, and quotient groups (Larsen, Johnson, \& Bartlo, 2013). Cook (2012) has designed LIT's reinventing of various concepts in ring theory including ring, integral domain, and field by leveraging the context of equation solving. Work has also begun in the area of advanced calculus with focus on topics such as the reinvention of the formal definition of limit (Swinyard, 2011), sequence convergence, completeness of the real numbers, and continuity of real functions (Strand, 2016). The findings from the overarching study 
reported on here will build on many of the findings from these efforts, and add to this pre-existing body of work with the addition of a new LIT.

Local instructional theories are generalized sequences of steps identified as key during the reinvention process of a particular mathematical concept (Gravemeijer, 1998). A local instructional theory differs from an instructional sequence in that its focus is not on the sequence itself, but on providing a rationale for the sequence (Larsen, 2013). While LITs do include a sequence of generalized tasks, more importantly they also include the theoretical and empirical rationale for each task. This rationale helps to explain how the necessary mathematics might emerge in the student's informal approaches. Another purpose of including the rationale is so that a future user of the LIT can figure out how to implement it in their context. However, the current RME literature does not explain in detail how to do this; how to use the rationale to adapt a generalized sequence to specific situation, and that is what this paper is about.

\section{Design Study Methods}

The findings reported here are from a larger design experiment aimed at developing a local instructional theory for the guided reinvention of a classification system for symmetry groups in the context of chemistry. Three teaching experiments were conducted with pairs of students in order to test and refine a theory for how students might reinvent a flowchart or decision tree that can correctly identify the symmetry group of a given molecule (see Chapter 5: Paper 1: Developing an Active Approach to Chemistry Based Group TheoryBergman \& French, 2019). My job as the instructional designer was to both evoke various student strategies and also find was that those 
strategies could be leveraged into the development of more formal mathematics. My consistent effort to use of students' own mathematics was intended to support students sense of ownership over mathematics as suggested by RME (Gravemeijer, 1998). The main research question guiding this study was: How can students be supported in reinventing an algorithm for the classification of chemically important symmetry groups?

The teaching experiments were conducted with pairs of students with varying mathematical backgrounds. While the ultimate goal was to create a LIT for undergraduate students, I chose to conduct the first teaching experiment (TE1) with a pair of mathematics education graduate students, who had extensive experience studying abstract algebra. The participants in TE1 had both completed a yearlong graduate level abstract algebra sequence including a ten-week investigation into the classification of finite groups. The mathematical activity of the students in TE1 informed a preliminary local instructional theory that was then tested in the subsequent teaching experiments. A detailed account of their activity can be found in Bergman \& French, 2019. While the results of TE1 provided important insights on the LIT overall, the experiment didn't supply insight into the additional support students with less mathematics might need. Therefore, additional teaching experiments were always assumed to be necessary. Teaching experiment two (TE2) involved a pair of senior level undergraduate students who had recently completed a traditional proof-based introductory group theory course and were given the pseudonyms Arthur and Stu. The third and final teaching experiment was conducted with Ada and Sophie (pseudonyms) majoring in mathematics and engineering, respectively. Ada and Sophie had recently completed an introductory linear 
algebra course and neither had any experience with group theory or advanced

mathematics. The data reported here is from TE2 and TE3 exclusively.

The LIT was ultimately abstracted from the mathematical activity of the students across all three teaching experiments and is described as the 6 steps that were identified as key in each of the three reinvention processes, seen in Table 8 below. The steps of the LIT are described in terms of students' mathematical activity, and further elaborations of each step along with sample prompts and evidence of students' mathematics can be found in Chapter 6 Manuscript 2. This paper will focus on Step 2 of the LIT in which students develop group representations compact enough to serve as outputs of their classification system.

Table 8. A local instructional theory for the guided reinvention of a classification algorithm for chemically important symmetry groups

\begin{tabular}{|c|c|}
\hline Step & Rational/Purpose \\
\hline $\begin{array}{l}\text { 0. An experientially real } \\
\text { starting point in the } \\
\text { context of geometric } \\
\text { symmetry where } \\
\text { students are introduced } \\
\text { to the idea that a } \\
\text { symmetry can be } \\
\text { described as a rigid } \\
\text { motion that can be } \\
\text { combined with another } \\
\text { symmetry to produce a } \\
\text { (possibly new) } \\
\text { symmetry. }\end{array}$ & $\begin{array}{l}\text { The idea that a symmetry can be described as a rigid motion that can be } \\
\text { combined with another symmetry to produce a (possibly new) } \\
\text { symmetry must be experientially real to students. This is important } \\
\text { because in order to do group theory the elements of the group, in this } \\
\text { case the symmetries, must be able to be combined under some kind of } \\
\text { operation. Symmetries as physical properties, such as planes of } \\
\text { reflection or as orientations from the result of a rotation don't make } \\
\text { sense to combine. However a reflection about a plane followed by a } \\
\text { particular rotation is absolutely reasonable. Students need to also have } \\
\text { some understanding that symmetries can be collected into groups. This } \\
\text { may require preliminary instructional activities. }\end{array}$ \\
\hline
\end{tabular}




\begin{tabular}{|c|c|}
\hline $\begin{array}{l}\text { The emergence of a } \\
\text { strategy for representing } \\
\text { the symmetry group of a } \\
\text { given molecule as a } \\
\text { model-of the symmetry } \\
\text { group of a particular } \\
\text { molecule (Model 1) }\end{array}$ & $\begin{array}{l}\text { The reinvention of a classification system for determining symmetry } \\
\text { groups of various molecules should begin with students' informal } \\
\text { activity in an experientially real context. The students initial process for } \\
\text { identifying and describing the symmetry elements of the molecules as a } \\
\text { group (Model 1) can be seen as anticipating the process that they will } \\
\text { eventually formalize and incorporate into something like the standard } \\
\text { flow chart (Model 2). This activity should anticipate both the } \\
\text { uniqueness of a symmetry group for each molecule based on the } \\
\text { collection of symmetries that exist within the molecule, and it should } \\
\text { expose students to a variety of group structures; such as } \mathbb{Z}_{2} \times \mathbb{Z}_{2} \text {, a } \\
\text { dihedral group, and the direct product of a dihedral group and } \mathbb{Z}_{2} \text {. At } \\
\text { this point the emergence of a system to determine symmetry groups, } \\
\text { (Model 2) is only seen by the researcher as a model of the student's } \\
\text { informal activity related to identifying particular symmetry groups. }\end{array}$ \\
\hline $\begin{array}{l}\text { 2. Describing and naming } \\
\text { the symmetry group with } \\
\text { an encapsulated group } \\
\text { representation as a } \\
\text { model-for the symmetry } \\
\text { group of a molecule } \\
\text { (Model 1) }\end{array}$ & $\begin{array}{l}\text { As students begin to develop a system for identifying various symmetry } \\
\text { groups, they need to decide on a group representation that is } \\
\text { encapsulated or compact enough to be used as the outputs for their } \\
\text { classification system. The students' encapsulated group representation } \\
\text { can be seen as the final version of the students' model for their unique } \\
\text { symmetry group (Model 1), which again will be incorporated into their } \\
\text { classification system (Model 2). This is an initial step in the transition } \\
\text { from model of to model for. }\end{array}$ \\
\hline $\begin{array}{l}\text { 3. Formulating an explicit } \\
\text { system for determining } \\
\text { the symmetry group of a } \\
\text { molecule (Model 2) as } \\
\text { mathematizing the } \\
\text { previous activity }\end{array}$ & $\begin{array}{l}\text { A system for determining the unique symmetry group for a given } \\
\text { molecule, which initially emerged as a model of the students' informal } \\
\text { activity, is explicated and then used as a model for more formal activity } \\
\text { as it feed into a classification system for determining the symmetry } \\
\text { group of any given molecule (Model 2). }\end{array}$ \\
\hline $\begin{array}{l}\text { 4. "Applying" the system } \\
\text { for determining the } \\
\text { symmetry group of a } \\
\text { specific molecule } \\
\text { (Model 2) in similar } \\
\text { situations }\end{array}$ & $\begin{array}{l}\text { The transition of a classification system (Model } 2 \text { ) from a model of the } \\
\text { students' activity to a model for more formal reasoning can begin by } \\
\text { having the students consider similar contexts by investigating more } \\
\text { molecules. Students can be given a variety of molecules whose } \\
\text { symmetry groups are both the same and slightly different than what } \\
\text { they saw in Step } 1 \text {. By considering new molecules with very similar } \\
\text { group structures to the ones they have already investigated, say } \mathrm{D}_{8} \times \mathbb{Z}_{2} \\
\text { instead of } \mathrm{D}_{6} \times \mathbb{Z}_{2} \text {, students can test their system. By considering } \\
\text { something new, such as a pure cyclic group, students can also refine } \\
\text { their system to include more group structures. }\end{array}$ \\
\hline
\end{tabular}




\begin{tabular}{|l|l|}
\hline $\begin{array}{l}\text { 5. "Applying" the system } \\
\text { for determining the } \\
\text { symmetry group of a } \\
\text { specific molecule } \\
\text { (Model 2) in different } \\
\text { contexts }\end{array}$ & $\begin{array}{l}\text { This transition of the classification system to a model of a classification } \\
\text { system for chemically important symmetry groups (Model 2) which can } \\
\text { be used for more formal reasoning can continue by having the students } \\
\text { consider different but structurally similar contexts. This is } \\
\text { accomplished in two different ways: } \\
\text { a. Students are first asked to test their model on a molecule that has a } \\
\text { symmetry group that students already have experience with, but which } \\
\text { can be generated by new kind of symmetry that they have not } \\
\text { experienced, an inversion. In doing so, students can continue to refine } \\
\text { their model to include more kinds of symmetries and/or more kinds of } \\
\text { symmetry groups. }\end{array}$ \\
$\begin{array}{l}\text { b. Students are then asked to test their model on very symmetric } \\
\text { molecules with high order symmetry groups. While these types of } \\
\text { molecules have new symmetry groups than those the students have } \\
\text { previously experienced, they are composed of the same symmetry } \\
\text { elements the students have already used in their model. }\end{array}$ \\
\hline $\begin{array}{l}\text { Justifying generality / } \\
\text { robustness of the } \\
\text { classification system } \\
\text { (Model 2) }\end{array}$ & $\begin{array}{l}\text { Students should know that one aspect of a complete classification } \\
\text { system is one in which they can convince someone else that their } \\
\text { flowchart can accurately identify the symmetry groups of any three- } \\
\text { dimensional molecule. Students' arguments for the generalizability of } \\
\text { their classification system may be quite different depending on their } \\
\text { level of mathematical background. }\end{array}$ \\
\hline
\end{tabular}

The students' activity in Step 2 varied greatly depending on their mathematical background, especially comparing TE2 and TE3. The goal of this paper is to highlight these variations and how I responded to these variations in order to support the students in completing Step 2. For the students in TE2 who had previous exposure to conventional group theory concepts, their goal for Step 2 was to use "standard group names." By "standard name" I am referring to the paradigmatic representatives of the isomorphism classes to which each symmetry group belongs. For example, there is only one algebraic structure that satisfies all of the group axioms with three elements. This isomorphism class is often referred to as $\mathbb{Z}_{3}$ even though the elements in the set are often referring to something other than $\{0,1,2\}$ under modular addition. It is common practice in abstract algebra, and also in chemistry, to use a single name to describe an entire isomorphism class. In chemistry they do it quite explicitly by saying ammonia belongs to 
$D_{6}$. While the students in TE2 seemed to have some access to standard group names, they needed additional support connecting their intuitive notions of different group structures with conventional representations.

Recall that the students in TE3 (teaching experiment 3) no previous exposure to abstract algebra and so standard group names were simply unavailable to them. Instead of developing tasks to strengthen their connection to conventional group theory, I developed a series of additional tasks that led them to reinvent group names altogether. Since the entire LIT requires that students can describe a set of symmetries as a symmetry group, the students in TE3 were first introduced to the group concept at the start of the experiment in order to productively engage in the LIT. When introduced to the group concept the students became familiar and proficient with using a particular group representation called a group presentation. A group presentation consists of a list of generating element, their orders, and the relations between each pair of generators. For example the symmetries of a triangle $D_{6}$ can be represented as $\left\{R F \mid R^{3}=F^{2}=I ; R F\right.$ $\left.=F R^{-1}\right\}$ which is a kind of shorthand recipe, or "list of rules" necessary to recreate all the elements in the group and see how they combine under the operation.

In both TE2 and TE3 students were eventually asked to come up with the names of symmetry groups of particular molecules which needed to be concise enough representations to be used as an output in a flowchart or decision tree. However, neither of the groups could consistently produce group names on their own, and due to the mathematical backgrounds of the students, different mathematics was available to them in order to solve the problem. Therefore two design challenges emerged 1) for students with limited access to conventional group theory, and 2) for students with no access to 
conventional group theory. This paper explains how I as the instructional designer responded to both of these situations in order to support the students in successfully completing Step 2. The main research question leading this activity and framing the findings of this paper is: How do I support students in successfully engaging in the mathematical activity necessary for the LIT while building on the strategies that they offer and the mathematics available to them? The results of this specific question might also speak directly to our broader understanding of how to use LITs with varying populations of students and begin to answer the bigger research question of: How are LITs adapted into different instructional sequences depending on the mathematical background of the student?

\section{Data Analysis Methods}

The corpus of data included video recordings of each teaching experiment and all the written work which was scanned into pdf documents. The two teaching experiments reported here, TE2 and TE3, were just shy of 17 hours and 13 hours, respectively where TE3 consisted of 12 sessions and TE2 11 sessions total. Across both TE2 and TE3 the sessions ranged from 35 to 124 minutes, averaging 78 minutes per session across both experiments. Between sessions I maintained a document recording both the goals for each session, and a short recap of the students' mathematical activity after each session including a description of how well the goals were met as a part of ongoing analysis. Once each experiment was complete the video recordings were used in retrospective analysis. For each session, the video was first trimmed into 3 minute codable units, then each codable unit was analyzed and the analysis was organized into a content log (seen in 
Table 9 below). The content logs were used to record a description of the students' activity, highlighted places the students moved forward in the LIT, and ultimately helped organized my understanding of the student's activity overall.

Table 9. Example of a content log used for data analysis and organization.

\begin{tabular}{|c|c|c|c|}
\hline $\begin{array}{l}\text { Video } \\
\text { Clip }\end{array}$ & What happened & $\begin{array}{c}\text { Particularly interesting } \\
\text { things }\end{array}$ & $\begin{array}{l}\text { Ways in which the } \\
\text { students moved their } \\
\text { thinking forward }\end{array}$ \\
\hline $\begin{array}{l}\text { TE1_S1 } \\
57 \text { to } 60\end{array}$ & $\begin{array}{l}\text { Stu says he's not concerned because he thinks that it } \\
\text { just has to do with how they labeled e to begin with. } \\
\text { I remind them that we're trying to find groups of } \\
\text { symmetries instead of groups of transformations, so } \\
\text { when we combine these things don't we expect certain } \\
\text { things to happen. } \\
\text { [I think that I'm trying to get them to see that they } \\
\text { need a homomorphism, where I should have asked } \\
\text { them do they have all the same kinds of symmetries } \\
\text { and do they work together in the same way in both } \\
\text { groups.] } \\
\text { Arthur asks about structure preservation and writes } \\
\text { down the following, } \\
f(X Y)= \\
\quad f(X) f(y) \\
\text { I ask what it means for two groups to be the same, or } \\
\text { equivalent? } \\
{[00: 01: 16.23] \text { Stu says, "no I mean that this is a }} \\
\text { homomorphism". } \\
\text { I immediately ask what he means by homomorphism, } \\
\text { he has an elaborate explanation, super interesting. }\end{array}$ & $\begin{array}{l}00: 01: 16.23] \text { Stu says, "no I } \\
\text { mean that this is a } \\
\text { homomorphism". } \\
\text { I immediately ask what he } \\
\text { means by homomorphism, } \\
\text { he has an elaborate } \\
\text { explanation, super } \\
\text { interesting. }\end{array}$ & $\begin{array}{l}\text { I ask what it means for } \\
\text { two groups to be the same, } \\
\text { or equivalent? }\end{array}$ \\
\hline
\end{tabular}

In order to understand similarities and differences across each of the LIT's I created a table of all the prompts used across each of the three teaching experiments, found in Appendix B. This table allowed me to first see the consistency of the student's mathematical activity which was abstracted into the LIT described in Chapter 6 : Manuscript 2. The table also allowed me to distinguish situations where I did things differently from the previous $\mathrm{TE}(\mathrm{s}) ; 1)$ in expectation of differences in students' mathematical backgrounds and/or 2) in response to differences in students' mathematical 
backgrounds. This round of data analysis illuminated the stark differences between both the students' mathematical activity and my responses to that activity as the researcher during Step 2 of the LIT during TE2 and TE3. This paper is a detailed description of the students' mathematical activity while completing Step 2 of the LIT during TE2 and TE3 and how I as the researcher responded to their activity in an attempt to build on their intuitions and support their success.

In order to complete Step 2 of the LIT, students needed to identify the symmetry groups for a variety of molecules AND describe each group with some kind of representation that was encapsulated enough to eventually serve as an output for the student's system for finding symmetry groups. In other words the students needed to assign something like a group name to the different types of symmetry groups. In both TE2 and TE3, additional prompts and tasks were used to help support students in identifying/creating these group names. The nature and goals of the supplemental tasks and prompts were very different in each of the teaching experiments reflective of the student's mathematical backgrounds. In both instances I, the teacher researcher, responded to the student's mathematical activity by providing supplemental tasks. Each task aimed to capitalize on the observed activity and then leverage their strategy into an approach that was ultimately robust enough to successfully complete Step 2. Below are two different cases of how students were supported in meeting this goal.

Results

The results of these analyses are presented below in two cases. Each case describes; the students' initial approaches to using group representations, additional prompts and/or 
tasks used to support further refinement of their group representation, and lastly how the students engaged with the additional tasks to ultimately complete Step 2 . Case 1 is a tale of partial successes and lessons learned while working with Arthur and Stu in TE2 who had limited access to conventional group theory. Case 2 is a success story highlighting the work of Ada and Sophie in TE3.

Case 1: Arthur and Stu

To begin TE2 Arthur and Stu were asked to first rank the four molecules found in Figure 56 below from most to least symmetric. Unfortunately, Arthur and Stu had admittedly "rough ideas" when it came to symmetry and were somewhat overwhelmed by the possibilities of three-dimensions. After a quick conversation about symmetries and symmetry equivalence in two-dimensions Arthur and Stu returned to threedimensional space to ultimately find the symmetry group of each of the molecules in Figure 56.

\begin{tabular}{|c|c|c|c|}
\hline \multicolumn{2}{|c|}{ Prompt: Find the symmetry group for each of the following: } \\
\hline Water & Ammonia & Tetra-aza copper II & Ethane (eclipsed) \\
\hline
\end{tabular}

Figure 56. The initial prompt given to participants in TE2.

Arthur and Stu had previous experience with group theory and therefore favored standard group names such as $\mathbb{Z}_{2} \times \mathbb{Z}_{2}$ and $D_{6}$; again names that they had studied or at least been previously exposed to in their formal education. This was not surprising 
considering all the possible kinds of group representations, group names are the most common representation used in abstract algebra texts (Bergman, Melhuish, \& Kirin, 2015). So, while the students did have some access to group names they always seemed to be at the tip of their tongues. From the very beginning Arthur and Stu's strategy was to try and identify a "known group" (represented by a standard group name) that was the same as whatever symmetry group they had just created through observations, only with differently labeled elements. For example, while identifying their first symmetry group for a water molecule, Arthur and Stu made a Cayley table to organize their work, seen in Figure 57.

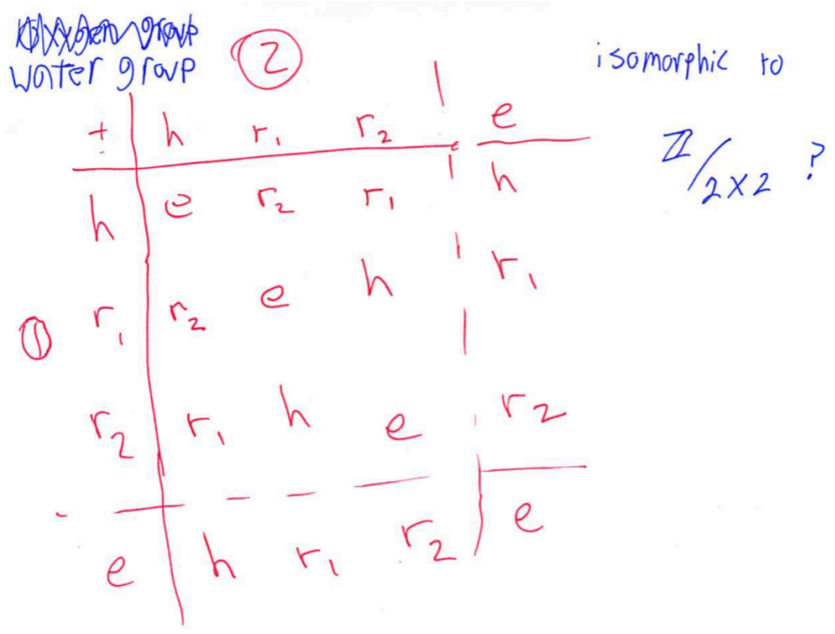

Figure 57. Arthur and Stu's Cayley table for the symmetries of water

Once they had completed their table Arthur mentioned that their symmetry group for water "feels a lot like the set $\{(0,0),(0,1),(1,0),(1,1)\}$ under some kind of operation," then suggested $\mathbb{Z} / 2 \times 2$ as seen written in Figure 57 above. While one might argue Arthur was close in his description, the symmetry group for water is actually isomorphic to $\mathbb{Z}_{2} \times \mathbb{Z}_{2}$. When I as researcher asked if maybe he meant $\mathbb{Z}_{2} \times \mathbb{Z}_{2}$ instead, Arthur and Stu 
both immediately recognized the more familiar group name and we moved on to finding the symmetries of the remaining molecules.

Later on, when working on ethane Arthur and Stu employed a similar strategy of first identifying all the possible symmetries as resulting orientations, which they referred to as "states" leaning on the one-to-one correspondence between number of unique symmetries and unique orientations. Once they had identified twelve different symmetries Arthur and Stu were asked if they could identify which group they had found. While $\mathbb{Z}_{2} \times \mathbb{Z}_{2}$ and the symmetries of triangle, the isomorphic names they used for the symmetry groups of water and ammonia respectively, were arguably within the example space of known groups that Arthur and Stu had experience with, it was clear that the group $D_{6} \times \mathbb{Z}_{2}$ was less familiar. Again, their goal was always to identify a "known group" that behaved the same as their symmetry group, Stu began by suggesting the following:

Stu: I'm inclined to put it (the collection of 12 symmetries) in a 144 sized table which is daunting.

Arthur: Doesn't that mean we should also look for a group that we, a known group with a...

Stu: Yeah

Annie: Ok wait, first of all does the group have 144 elements?

Stu: $\quad$ No it has 12.

Arthur: So when I said 144, I was picturing a 12 by 12 Cayley table.

Annie: Do you both feel pretty confident that this is a group of order 12 ?

Stu: Yes 
Arthur: Yes

Annie: That this has 12 symmetries?

Arthur: Yes

Annie: Ok, how do you want, so we're still looking for what group is this? There are a lot of groups of order 12, like 5 or 6 and they're kind of funky. Um, but there are different ways that you can represent that group, you can finish filling out your Cayley table, you can, um you know there's different ways that you can describe it. As long as you feel like you've given enough information.

At this point I was not sure what other kinds of group representations were available to Arthur and Stu that might help them to identify the conventional group name. As the conversation continued, I tried to steer the students away from needing outside sources and subtlety suggest group presentations. However, as it was their strategy, unsurprisingly Arthur continually expressed a desire to use conventional names, symbols, and representations whenever possible. In fact this was not only his approach to describing symmetry groups as a whole but was his desire throughout the entire teaching experiment.

Arthur: I am feeling good about twelve unique elements. So I think, I guess it really depends on how many groups of order 12 . If there is a number of them that's too hard to process and that we can't look for, because if we 
had, depending on how many order 12 groups there are, comparing Cayley tables would be relatively easy unless there are...

Annie: I think there are 5 or 6 groups of order 12.

Witness: There's 5 .

Arthur: That would be hard to generate and easy to look up.

Annie: Is there another way that we could describe this group? Like maybe instead of comparing him to some known group, we can just say, hey this is a group and here's what I know about it.

Arthur: We could do that.

Annie: Just because originally convinced that maybe this (their table for Ammonia) wasn't exactly the same group as the symmetries of a triangle, it didn't mean that you didn't feel like you had finished the group.

Arthur: Um hmm.

Annie: You know once you had a complete Cayley table, that was one way of describing this group.

Arthur: Right.

Annie: Is there another way you could describe a group? Maybe we have to do the Cayley table?

Arthur: I find the Cayley table helpful but also, matching it to like a $\mathbb{Z}_{2} \times \mathbb{Z}_{2}$ or matching it to some known ordered groups that mirror its behavior. Which is kind of the direction I was thinking in for comparing it to a Cayley table. What are you thinking? (to Stu) 
Arthur and I appear to be approaching an impasse, he wants to compare Cayley tables to outside information, and I don't want to incorporate checking an outside source as part of the LIT. As the design researcher, this exchange was beginning to show me an issue with the LIT, which ultimately led to the explication of Step 2; students with limited group theory knowledge might also have limited example spaces of groups, more importantly a limited example space of group representations. Eventually Arthur asks Stu to share his thoughts, and Stu's response was absolutely epic:

Stu: I think we could rep... like one way I might wanna think about starting is um, we know that we could compare it to this (ammonia) because this half of ethane looks an awful lot like ammonia, right? (see Figure 58 below)

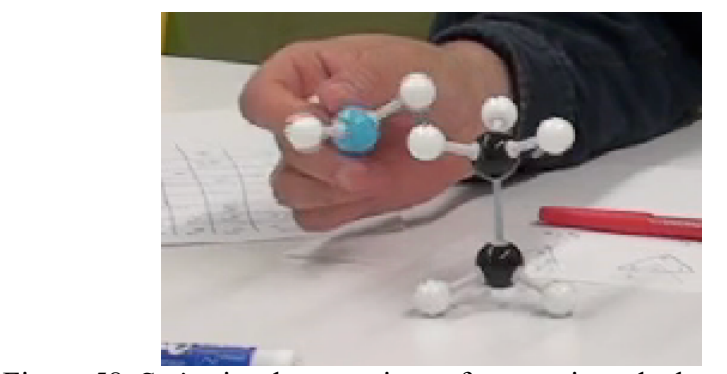

Figure 58. Stu's visual comparison of ammonia and ethane

Arthur: yeah

Stu: $\quad$ So I feel like it might be something like $\mathbb{Z}_{2}$ cross ammonia. If that makes any sense?

In the moment I interpreted this response as very similar to the conventional group name of $\mathbb{Z}_{2} \times D_{6}$ and so I pressed Stu to explain his thinking a bit further: 
Annie: What does $\mathbb{Z}_{2}$ cross something get you?

Stu: It would get us a direct product, well $\mathbb{Z}_{2} \ldots$ it would get us an order 12 group, well I don't know if it's a group but an order 12 set for sure Arthur: $\mathbb{Z}_{2} \times \mathbb{Z}_{6}$

Stu: Well that's not really $\mathbb{Z}_{6}$ is it (motioning to table for ammonia)?

Annie: This was actually $D_{6}$, so of order 6 there's $2, \mathbb{Z}_{6}$ and $D_{6}$. There's the commutative guy who's cyclic or you could think of him like $\mathbb{Z}_{2} \times \mathbb{Z}_{3}$ and then there's the $D_{6}$, right. The one where the order 3 and the order 2 are non-commutative. I think you probably found that in your dihedral group you had what's called the dihedral relation right? Where rotations times flip was equal to the inverse rotation times flip. Right. It wasn't RF=FR it was $\mathrm{RF}=\mathrm{R}^{-1} \mathrm{~F}$, right it was the little swippy swappy... does that sound...

Arthur: That sounds familiar, but I don't pretend to like... I didn't make that connection.

Annie: I like what you're thinking here on, maybe it's this guy (ammonia) pasted on something else. That's pretty clever.

Stu: I mean this is a set (this collection of symmetries for ethane) here for sure, and we have an operation of composition. So we could think of it as a group that way but we don't have a way of proving that, like with, like this is how I want to prove it (motions to the start of a Cayley table for ethane). This is like what feels concrete to me. The rest of this feels like speculation and I'd have to like really think about a clever way to prove that's true. 
Arthur: That's true. It also seems like we're going to need to come up with something clever to make that doable (points to Tetra-aza copper II molecule) cause I don't, I suspect that...

Stu: Don't touch it yet.

Arthur: ...this tool won't work for this (referring to ammonia). I'm prepared to be surprised but I'm worried that if we don't come up with something, some clever connection to a known group that we won't be able to extend it to this (Tetra-aza copper II).

Unsure of how to move forward without the use of outside Cayley tables to verify their group structure, in the moment I chose to step out of the room to discuss with my witness of how we might move forward without having to make a Cayley table to compare to known groups of order 12. Upon returning to the room Stu confidently said:

Stu: I feel confident that it's $\mathbb{Z}_{2} \times D_{6}$.

Annie: You do! Well that's a good place for me to walk in.

David: So why do you feel confident about that?

Stu: Because the purple triangles always match the green triangles below, and for every purple triangle there's a green triangle on top and that's a lot like $\mathbb{Z}_{2}$ to me (see Arthur and Stu's geometric representations for ethane in Figure 59 below). And then the $D_{6}$ for the other reason, this (ethane) looks like ammonia which we decided was $D_{6}$.

Annie: What do you think about that Arthur? 
Arthur: I follow that, that we're essentially looking at the symmetries on a triangle and now we're just giving it two, you know we're adding one more piece of information with two states, yeah. [Note that Arthur's use of "state" in this instance is describing the color associated with the top triangle and is different than before when a "state" referred to an orientation resulting from a symmetry transformation.]

Arthur: I'm seeing almost like ordered pairs where we have the symmetry from the dihedral group and then just a one or a zero to denote purple or green. Annie: Oh.

Arthur: Just cause we've got $D_{6}$ information paired with binary information, so that's what I meant by that.

Annie: And Stu does that jive with how you're thinking about it too?

Stu: Yes.

Annie: Alright, I feel like we should just say, yeah that's the group that you found. 


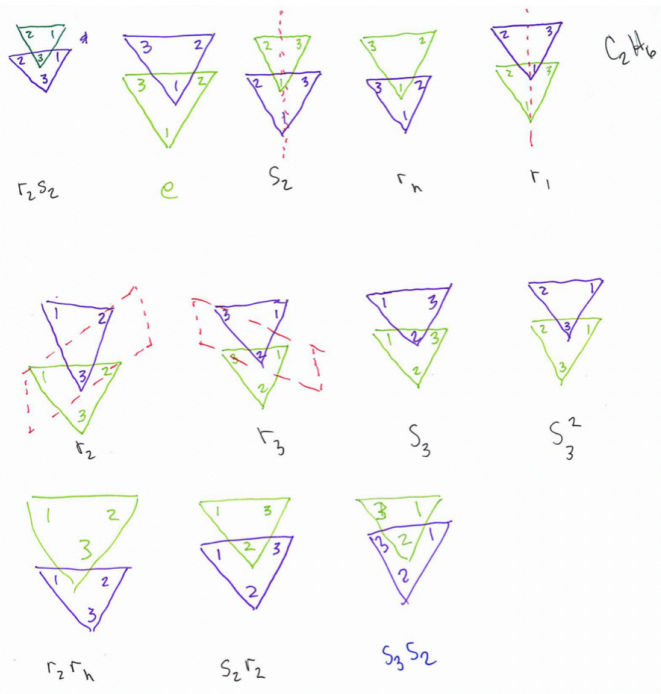

Figure 59. Arthur and Stu's geometric representations for the symmetries of ethane.

So our conversation about the symmetry group for ethane ended with me telling Arthur and Stu that they were correct, the symmetry group for ethane is something like $\mathbb{Z}_{2}$ cross ammonia, in fact it was isomorphic to $\mathbb{Z}_{2} \times D_{6}$. After the session, during ongoing analysis before the next session, we decided that this idea of $\mathbb{Z}_{2}$ cross ammonia was particularly interesting and worth exploring further. First, I wanted to know more about how Stu was thinking about direct products. The symmetry group the students constructed for ethane can technically be described as an internal direct product, Stu chose to use an external direct product to describe the group which is technically different. External direct products are groups represented by ordered pairs where the components in each position come from completely different groups where the operations can also be different. In general two elements of the direct product $G \times \mathrm{x} H$, say $(a, b)$ and $(c, d)$, are combined component wise, $(a, b)(c, d)=(a * c, b \circ d)$ where * is the operation in group $G$ which contains elements $a$ and $c$ and $\circ$ is the operation in group $H$ which contains elements $b$ and $d$. For example consider the group $D_{6} \times \mathbb{Z}_{2}$; the elements in the 
first component are symmetries of a triangle $\left\{F^{n} R^{m} \mid 0 \leq n \leq 1\right.$ and $\left.0 \leq m \leq 3\right\}$ combined under function composition and the elements in the second component are the either 0 or 1 combined under a specific modular addition.

Alternatively, internal direct products are a bit more subtle and are a more natural representation of the symmetry group Arthur and Stu were investigating. A group $G$ can be described as an internal or "inner" direct product if $G=H K$ where $H$ and $K$ are normal subgroups of $G$, such that $H \cap K=\{e\}$; that is every element $x$ of $G$ can be written as $x=$ $h k$ for some $h \in H$ and $k \in K$ and then $G \cong H \times K$. The trivial intersection guarantees that each element can be uniquely represented as a product of the form $h k$ where $h$ is an element of $H$ and $k$ is an element of $K$. The fact that both subgroups are normal means that a direct product of two such elements, $\left(h_{1} k_{1}\right)\left(h_{2} k_{2}\right)$ another such element. Specifically, $\left(h_{1} k_{1}\right)\left(h_{2} k_{2}\right)=\left(h_{1} h_{2}\right)\left(k_{1} k_{2}\right)$. This guarantees that an internal direct product $G=H K$ is isomorphic to the external direct product $H \times K$ (see Appendix C for a proof of the isomorphism). Therefore a molecule such as Tetra-aza copper II which has a pure rotational symmetry group isomorphic to $\mathbb{Z}_{4}$, could never be isomorphic to a direct product because it fails the very first condition. There does not exist subgroups with a non-trivial intersection in a pure rotational group, instead all the elements in the symmetry group can be represented by various powers of a single symmetry.

This commutative relationship between elements in the two subgroups is essential, otherwise instead of creating a direct product the symmetry group could instead become a semi-direct product. Take for example the two molecules ammonia which has a symmetry group isomorphic to $D_{6}$ composed of an order 3 rotation and a series of "vertical" reflection planes incident with the rotational axis and boric acid which has a 
symmetry group isomorphic to $\mathbb{Z}_{3} \times \mathbb{Z}_{2}$ composed of an order 3 rotational axis and a "horizontal" reflection plane orthogonal to the rotational axis, seen in Figure 60 below.

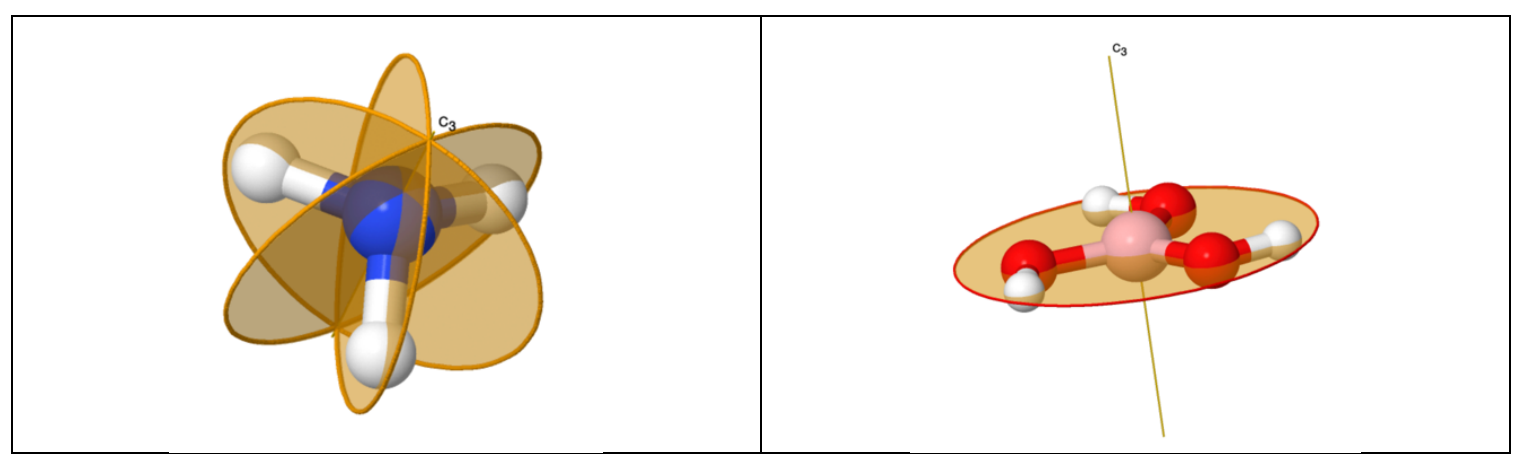

Figure 60. The symmetries of ammonia and boric acid, respectively.

Notice that all the symmetries of both ammonia and boric acid can be represented as a combination of a rotation and a reflection, $r^{n} \sigma$, with $n \in\{0,1,2\}$ and the particular orientation of $\sigma$ would change depending on the molecule. While the elements of each symmetry group can be written with "the same" inscriptions, the ways in which the elements in the subgroup generated by the rotation and the subgroup generated by the reflection interact is different depending on the orientation of the reflection planes. In ammonia where the reflection planes are incident with, or contain the entire rotational axis, there exists a dihedral relation between the generating elements where $r \sigma=\sigma r^{-1}$. In other words if one were to label the hydro atoms in ethane 1-2-3 in clockwise order, the labeling would reorder after any reflection is preformed leaving a labeling akin to 1-3-2. Alternatively in boric acid where the reflection plane is orthogonal to the rotational axis, the combination of rotation and reflection has a no effect on the labeling. In boric acid if one were to label the hydrogens 1-2-3 clockwise, they would always remain in the same ordering no matter the symmetry transformation preformed. Ultimately this reordering effect reflections have in ammonia where the elements do not commute leads to what's 
referred to as an internal semi-direct product as opposed to the direct product in boric acid.

The relationship between these two different group structures is non-trivial even for advanced students, and so it inspired me to also push on Arthur and Stu's ideas about isomorphisms. As for “ $\mathbb{Z}_{2}$ cross ammonia" mathematically it isn't accurate for a number of reasons; ammonia isn't technically a group, none of the elements in the symmetries of ethane are integers under modulo addition, and there is no external direct product, therefore I was especially curious of how Stu saw this group as "like" that of the symmetries of ethane.

In order to gain insight into how Arthur and Stu were thinking about direct products and isomorphisms I first created a prompt to probe their thinking. Once I had a better sense of how they were imagining direct products and seeing equivalence between groups, I design follow-up tasks to support their understanding and strengthen it to the point where they could ultimately be successful in continuing on in the LIT. The following is organized by Arthur and Stu's thinking around direct products followed by their activity around arguing isomorphism.

Arthur, Stu, and Direct Products

Arthur and Stu's thinking around direct product can best be described in two steps. These steps are closely associated with the necessary conditions for a group to be an internal direct product, and again therefore isomorphic to an external direct product see Appendix $\mathrm{C}$ for a proof. In order for a group to be an internal direct must meet three conditions: 
1. every element in the group must be able to be represented as the product $h k$ of elements from two different subgroups, $H$ and $K$

2. these two subgroups $H$ and $K$ must have a trivial intersection

3. these two subgroups $H$ and $K$ must both be normal, which implies they elements of $H$ and $K$ are commutative, that is $h k=k h$ (see Appendix $C$ for a proof)

However, there is another kind of group structure called an internal semi-direct product that also meets the first two criteria above, as seen in the example above with boric acid. Using excerpts of their work I will argue that both Arthur and Stu saw evidence of the some of the conditions being met in their symmetry group. Then I will use the data to show that Stu goes on to show that he seems to have all the connections necessary to meet the three conditions, he just doesn't have the language to make a more formal argument. Arthur on the other hand continues to show evidence that he never fully sees their symmetry group meeting all the requirements.

$\underline{\text { Step 1: Arthur and Stu see the symmetry group for ethane looks like an internal product }}$ In order to better understand Arthur and Stu's thinking on direct products in general and to start our conversation I began with the prompt in Figure 61 below.

Last time you said that the symmetry group of ethane was $D_{6}$ cross $\mathbb{Z}_{2}$ can you tell me, what's the $D_{6}$ and what's the $\mathbb{Z}_{2}$ ? Like where is $D_{6}$ and where is $\mathbb{Z}_{2}$ ?

Figure 61. Investigatory prompt into student thinking on direct products 1. 
Arthur and Stu responded by providing very similar explanation around their thinking about direct products as the previous session, this time using their geometric figures in Figure 59 as a part of their argument.

Arthur: $D_{6}$ was the configuration of the three kind of top hydrogen as we were looking at them and $\mathbb{Z}_{2}$ encoded which face was up. Or in the case of these colorings, just cause we needed a way to tell the two triangular faces apart. So it was a case of the six combinations and then like a binary dimension to that, which is what crossing $\mathbb{Z}_{2}$ encoded.

Annie: Does that jive with that you were thinking Stu?

Stu: Yeah, yep that's what I was thinking.

Annie: Ok.

Stu: I wasn't convinced until I noticed that we indeed had the same number of green combinations as purple combinations because of (inaudible) for the top.

Annie: So, I guess of the twelve things that you found, do you see $D_{6}$ in these twelve? Is $D_{6}$ in there?

Stu: Yeah, it's the number of combinations of 3,2,1 in the triangle.

Annie: So, but there's twelve combinations here (pointing to paper).

Stu: Right, there's six green ones and six purple ones.

Annie: So is one, like are all the green ones a $D_{6}$ and all the purple ones a $D_{6}$ ? Is that how you're seeing it?

Stu: That's how I'm seeing it. 
Arthur: Umm hmm.

Annie: Ok, ok.

Stu: Yeah. That's crossed with whether purple or green is on top.

This description of their symmetry group is actually quite encouraging. Their portioning of the set into two subsets, is powerful since the group $D_{6} \times \mathbb{Z}_{2}$ can easily be partitioned into two copies of $D_{6}$ where one has a 0 in all the second components and the other has a 1 in all of them. By describing all the symmetries as the product of something in $D_{6}$ and "either color-swapped or not" they seem to be capturing the notion that all the elements in the group can be represented as some $h k$ for two subgroups $H$ and $K$.

Later in the session as Arthur and Stu were starting to describe a system for identifying symmetry groups, Arthur further elaborated on this idea of color swapping being representative of a direct product and when doing so Stu shared an important insight:

Annie: So it sounds like the mapping is part of the story, right the getting from a $3-d$ thing to some kinds of 2-d representation. In two of your molecules you chose to use representations with numbers and twice you didn't. I mean you can standardize that for your system if you want. You're right you did do slightly different things per molecule, but maybe you want one system moving forward.

Arthur: I think it would be most efficient to go to the numbers to move forward.

Stu: Well it's not just, it's... 
Arthur: Plus coloring.

Stu: Plus coloring on both.

Arthur: Yeah

Stu: And the coloring um, represented one kind of symmetry and the numbers represented a different type of symmetry, an independent type of symmetry.

Arthur: Ok so the numbers plus coloring I think is accessible, this is, these (some of their notation for ethane) were just unique markings that already existed plus coloring, so numbers plus coloring.

Stu: I guess what I'm trying to say is um, for both of these, this one (water) and that one over there (ethane). Here (water) we have numbers (differentiating the hydrogens) and we have colors and that helped us figure out, in the end we were able to see the crossing or the combinatorial arguments because we chose one numbering system for one type of symmetry and another numbering system which is colors for the other type of symmetry.

Arthur: Umm hmm.

Stu: Does that make sense what I'm saying?

Arthur: It does. Like, we could easily represent both of these (water and some of their markings for ethane) things just as numbers instead of dot and $\mathrm{x}$, and $\mathrm{o}$ and line and 1 and whatever.

Stu: $\quad$ But for the ethane the purple and green helped us see $\mathbb{Z}_{2}$ separate from $D_{6}$. Arthur: Um (agreeing) 
Stu: And for water, this one was $D_{6}$ cross, I can't remember

Arthur: $\mathbb{Z}_{2}$

Stu: $\quad \mathbb{Z}_{2}$

Arthur: $\mathbb{Z}_{2} \times \mathbb{Z}_{2}$.

Stu: Yeah and that helped us see that there was a direct product happening rather than just a $C_{4}$ like this (pick up Tetra-aza copper II).

Arthur: Yeah, it was like what's a group that has you know one two state, I'm gonna refine this. $\mathbb{Z}_{2} \times \mathbb{Z}_{2}$ you have a two-state piece of information crossed with another binary piece of information we saw that that was going on here (motions to water). We had binary coloring, binary hydrogen position.

Stu's explicit description of the coloring and the number representing different "independent" symmetries is important because that is a very important component of what makes their symmetry group an internal direct product instead of an internal semidirect product. Stu's attention to independence can be interpreted as evidence of him being at least implicitly aware of the importance of the commutativity of $r_{h}$ (the horizontal reflection associated with their "color swap") with the other symmetries. This symmetry is independent as in it doesn't have an impact on the orientation, which again is a function of commutativity. The thing Stu is seeing is important because it is the consequence of this commutativity. Which again establishes the equivalence between their internal direct-product and the external direct-product they used to describe their symmetries. An even early instance of Stu's recognition of this phenomenon was upon 
my returning to the room after he had first mentioned ammonia x $D_{6}$, Stu said, "Because the purple triangles always match the green triangles below, and for every purple triangle there's a green triangle on top and that's a lot like $\mathbb{Z}_{2}$ to me." While a strong argument can be made that Stu is observing the necessary conditions for an internal direct product to be met, it is also clear that he lacks the formal language necessary to describe why what he is observing is the necessary condition for this to have the proper group structure.

Later on while the students were trying to argue whether ethane was isomorphic to $D_{6}$ $\mathrm{x} \mathbb{Z}_{2}$ I asked them once more about direct products really specifically pushing on their description of a direct product as a group with an additional binary option. My goal was continue to gain insight on their thinking of direct products and to push on their descriptions direct products as a binary option like top and bottom. Very early in the teaching experiment the I had the students work with a set of 2-dimensional figures in order to help establish definitions for both symmetry and symmetry equivalence. One of the figures the students considered was triangular with a symmetry group of $D_{6}$, and one of the students' ways of describing the symmetries of this figure was as having three rotations then the option to flip the figure over and rotate three more times.

So way back when (in 2-space), one of the first ways you described the symmetries of a triangle was with an order 3 rotation then you flipped it over and could rotate it three more times. So it was kind like you had this order 3 rotation with the binary option of top up or bottom up, why then didn't we describe the symmetry group for a triangle as $C_{3} \times \mathbb{Z}_{2}$ ?

Figure 62. Investigatory prompt into student thinking on direct products 2.

In order to best understand the students thinking on direct products as a group with a binary option, I provided them with the prompt above. Stu's reaction was to immediately grab an ammonia molecule and marked the hydrogen atoms 1, 2, 3, considering it as if it 
were a two-dimensional triangle. He started twisting the molecule about a single bond between his fingers and starts trying to articulate his thinking captured in the except below:

Stu: When we reflect it (ammonia), the 1 stays stationary, and the 2 and the 3 change positions. When we rotate it, same thing happens, so I think it has something to do with the idea that.... yeah, on one side the numbers are clockwise and the other side they're counterclockwise and I think that's the issue. We don't have a mirror of orientation when we do the reflection, or not a mirror I shouldn't use that word. We don't have the same orientation when we do the reflection, we have a different orientation. I think it has something to do with that. Are you following what I'm saying?

Arthur: Not fully.

Stu: So this side has counterclockwise, on this side, (he turns over ammonia) it's clockwise.

Arthur: Um hmm.

Stu: I think the problem is in there somewhere, but I don't know why exactly. I think if I expected it to be $C_{3} \times \mathbb{Z}_{2}$ then I would expect them to have the same ordering on each side after the reflection. Um so, I'm not 100 percent convinced but that is what I'm thinking.

Stu's thinking actually captures the what's going on pretty well. He is correct that if one imagines ammonia as planar, then a 2-dimensional reflection about one of the bonds 
would be equivalent to a 3-dimensional rotation about the bond, both of which result in a reordering of the labels. As described earlier this reordering of the labels is a direct result of the semi-direct relation between the rotation and the reflection. There are many encouraging aspects of Stu's description of an informal way of checking for a semi-direct versus a direct product and his description is evidence that he is actually on top of the phenomenon he is trying to describe.

Ultimately, the students ended up going a different direction for a while where Arthur offered a different idea about the symmetries of a triangle:

Arthur: I kind of feel like if we had to start over with that triangle, the 2dimensional with the transparency, like we probably would turn it into $C_{3}$ $\mathrm{x} \mathbb{Z}_{2}$.

Stu: $\quad$...yeah maybe we could...

Arthur: Cause we've had a little practice thinking about you know what, what sort of group to use to classify certain types of information. We already know $D_{6}$ matches all the triangle stuff. You know now if you just have another side binary state, like which side is up or what color it is, crossing $\mathbb{Z}_{2}$ now feels like a very sensible like accessible procedure to grab. But I was reminded, I think you came up with that. That was part of some of the exploration we did like over time after we had messed with the triangle and everything. I think we were still thinking about states too, like we were thinking about ok, I have 3 states created by the rotation, and then I can create the other 6 states by flipping it over and then doing that same 
rotation. But maybe we're just better attaching the group theory to it now which is why we didn't do that cross $\mathbb{Z}_{2}$ thing to that triangle. Cause that was kind of the question, right?

Annie: Yeah.

Arthur: What did we do differently now?

Annie: Yeah. Or I mean, not necessarily why did you do it then, but could this (ammonia) be $C_{3} \times \mathbb{Z}_{2}$ ? Like is that a reasonable way to think about the triangle? It seems similar to how you're thinking about ethane so I'm just trying to see if this $\mathbb{Z}_{2}$ is sort of showing up in two places?

Stu starts by writing out two "interesting" elements that they would expect to find in $C_{3} \times \mathbb{Z}_{2},(1,1),(2,1)$. [Note: at this point in the interview we are being quite sloppy in our language and notation. We are talking about $C_{3}$ the order 3 group under multiplication and writing elements as if they are coming from $\mathbb{Z}_{3}$ the integers $\{0,1,2\}$ under addition mod 2.] He combines them to find that their product would be $(0,0)$ and then starts working on a map that might give the similar results, see Figure 63 below. While describing his map he puts rotations together and the reflections together pointing out, that while the rotations are similar to $C_{3}$ the reflections are not. He notices that the reflections are not similar to $C_{3}$ because order of the elements don't necessarily match up, however he keeps going, admitting all the while that he's really just exploring things. 


$$
\begin{aligned}
& (2,1)+(1,1) \in C_{3} \times \mathbb{/} / 2 \\
& (0,0)
\end{aligned}
$$

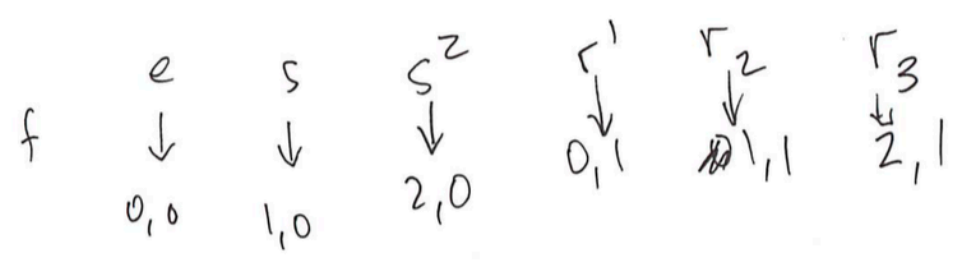

Figure 63. Arthur and Stu's mapping from $\mathrm{D}_{6}$ to " $\mathrm{C}_{3} \times \mathbb{Z}_{2}$ "

Stu starts checking to see if combinations of elements in $C_{3} \times \mathbb{Z}_{2}$ that have been mapped to the reflections give them the same output as combinations of reflections. Starting with $r_{1} r_{1}$ they find it maps to $(1,0)(1,0)$ which gives $(0,0)$ in $C_{3} \times \mathbb{Z}_{2}$ this is encouraging to Stu because he is hoping that each of the reflections when combined with themselves gives the identity. However encouragement quickly subsided when he combined $r_{2} r_{2}$ as elements in $C_{3} \times \mathbb{Z}_{2}$. Stu exclaimed when considering the combination $(1,1)(1,1)$, "here we'd get $(2,1)$ (technically he should get $(2,0))$ and that's not the identity which feels like a problem to me." Stu correctly concludes by saying, "either this bijection doesn't work, or they're not isomorphic." At this point Stu has shown both powerful and productive approaches to making an argument that their symmetry group can't possibly be $C_{3} \times \mathbb{Z}_{2}$, however again he lacks the formal language. Stu has all the right connections he just hasn't developed the ideas to the point that he can articulate them with confidence. While it is true that isomorphisms preserve order and that the order of a product of elements is equal to the least common multiple of the order of the elements, that is $|h k|=l \mathrm{~cm}(|\mathrm{~h}||\mathrm{k}|)$, Stu doesn't seem to have ready access to either of these "facts" at the moment at least not enough access to use them confidently. 
Arthur on the other hand has a different issue, he is still convinced that Stu's findings might just be the result of another jumbling issue. Recalling that when they were working with $D_{6}$ the product of two reflections was always a rotation and wonders if the thing is true in $C_{3} \times \mathbb{Z}_{2}$. Stu reiterates that it really bothers him that $r_{2}^{2}$ as represented in $C_{3} \times \mathbb{Z}_{2}$ isn't $(0,0)$, saying "it seems like a deal breaker to him." Stu goes on to explain that that he knows in ammonia $r_{2}^{2}$ would give $e$, but he's also intrigued by Arthur's "reflection-reflection is a rotation" idea. Stu considers $r_{2} r_{3}$ in $C_{3} \times \mathbb{Z}_{2}$ gives the identify and describes the result as weird. At this point Stu seems very convinced that they are different groups, saying that "the rotations match up but the reflections are all wrong." Stu explains his thinking:

Stu: I don't know why but it doesn't seem like they have enough of the same structure to call them isomorphic. I think this is a big problem right here (pointing to $r_{2}^{2} \neq 0$ ). It's possible that there's a function that I'm not seeing that would work better, but I know I want $r_{\mathrm{n}}^{2}$ to be zero, I know I want these to be...(motions to reflections). And this one cubed and this one cubed (motioning to the rotations) should be $e$ as well... One thing here (motions to ethane) that's different though is we have here (points to $r_{2}$ on their geometric inscriptions for ethane). Here we have 1-2-3 purple on top and (points to $s_{3} s_{2}$ (equivalent to $r_{2} r_{h}$ or $r_{2}$ with a color swap)) 1-2-3 both going clockwise with green on top. Do we have our map for ammonia...

[I scramble to grab their geometric inscriptions of ammonia.] 
Stu: So here we don't have that because it goes 1-2-3 then 1-3-2. But we don't have this repetition of ordering in the same order, 3-1-2, 3-2-1, you know they've it's like they're got twisted with just a triangle but for whatever reason maybe because they're mirror images of one another it's less twisted for this group (ethane) and so that separation seems to be working out. I don't know why.

Stu's use of "mirror images" to describe the direct product, again suggests that he might be on the path to noticing the main differences between these two groups is the orientation of the reflection that is resulting in either a semi-direct or direct product due to the horizontal reflection always being commutative.

After learning more about and reflecting on Arthur and Stu's descriptions of direct products, it became increasing clear that while they had some exciting thinking related to external direct products that might be leveraged to further develop their understanding of internal direct products, their description of the symmetry group also revealed that their understanding of isomorphisms might also need support. Their argument for how they were imaging $D_{6}$ and $\mathbb{Z}_{2}$ within $D_{6} \times \mathbb{Z}_{2}$ showed that they didn't have quite enough language to nail down an explicit isomorphism to a direct product, and so I came up with a plan to help them establish such an equivalence. In the process I hoped to also strengthen their understanding about isomorphisms with direct products so that they might feel more comfortable in a similar situation in the future. The following tasks were designed to meet this goal. Each of the supplemental tasks contains the prompt given to 
the students along with the goals for the task, how the students responded, and how the task might be used the same or differently with future participants.

Before I share each task in detail, it is important to note that throughout this portion of the teaching experiment Arthur and Stu's mathematical activity was somewhat disadvantaged by two things. First, even though they had symbols for their symmetries such as $s, r_{l}$, etc. they often reasoned with the configurations of the symmetries as per their inscription systems instead of as a motion, this is explain further with an accompanying example in the very first supplemental task. Second, the symbols that Arthur and Stu chose to use for their symmetries was extremely confusing. For instance their reflections in ammonia were simply labeled $r_{1}, r_{2}$, and $r_{3}$, symbols which gives no insight that these elements can also be written as combinations of rotations and a single reflection. This experience reinforced the power of having students represent symmetries as combinations of generates as Ada and Sophie were encouraged to do in TE3.

Pick six elements in ethane and prove that they are equivalent to $D_{6}$.

Figure 64. Supplemental task to support Arthur and Stu's thinking about isomorphisms 1

The first supplemental task was intended to gain insight on students' working understanding of isomorphism and to see how they might construct an isomorphism between groups. Since they had already claimed that there were multiple versions of $D_{6}$ contained within ethane, I chose to ask them to argue equivalence with $D_{6}$. Stu starts by saying, " So my guess is that we just pick either the purple ones or the green ones, cause that's what I've been thinking this whole time," but instead uses "the arrangements of the three numbers in the triangle" (his notion of $D_{6}$ ) to identify six elements of ethane 
attending to the labeling of the vertices. In order to match the orientation of triangles in their inscriptions systems, Stu starts by turning the ethane elements upside down so that they looked more similar to those in ammonia. He then begins to check the labeling of the vertices in ammonia elements and then finding the corresponding element in ethane that has the same vertex labels on its purple triangles. Stu puts big red boxes around all the them declaring them candidates for $D_{6}$ and labels an element in each set with A- F, seen in Figure 65. However, because the inscription systems have different starting orientations, this mapping system fails to preserve any group structure. Notice the very correspondence Stu created between the identity element in ammonia and a reflection in ethane, breaks a property of isomorphisms that identities must map to identities.

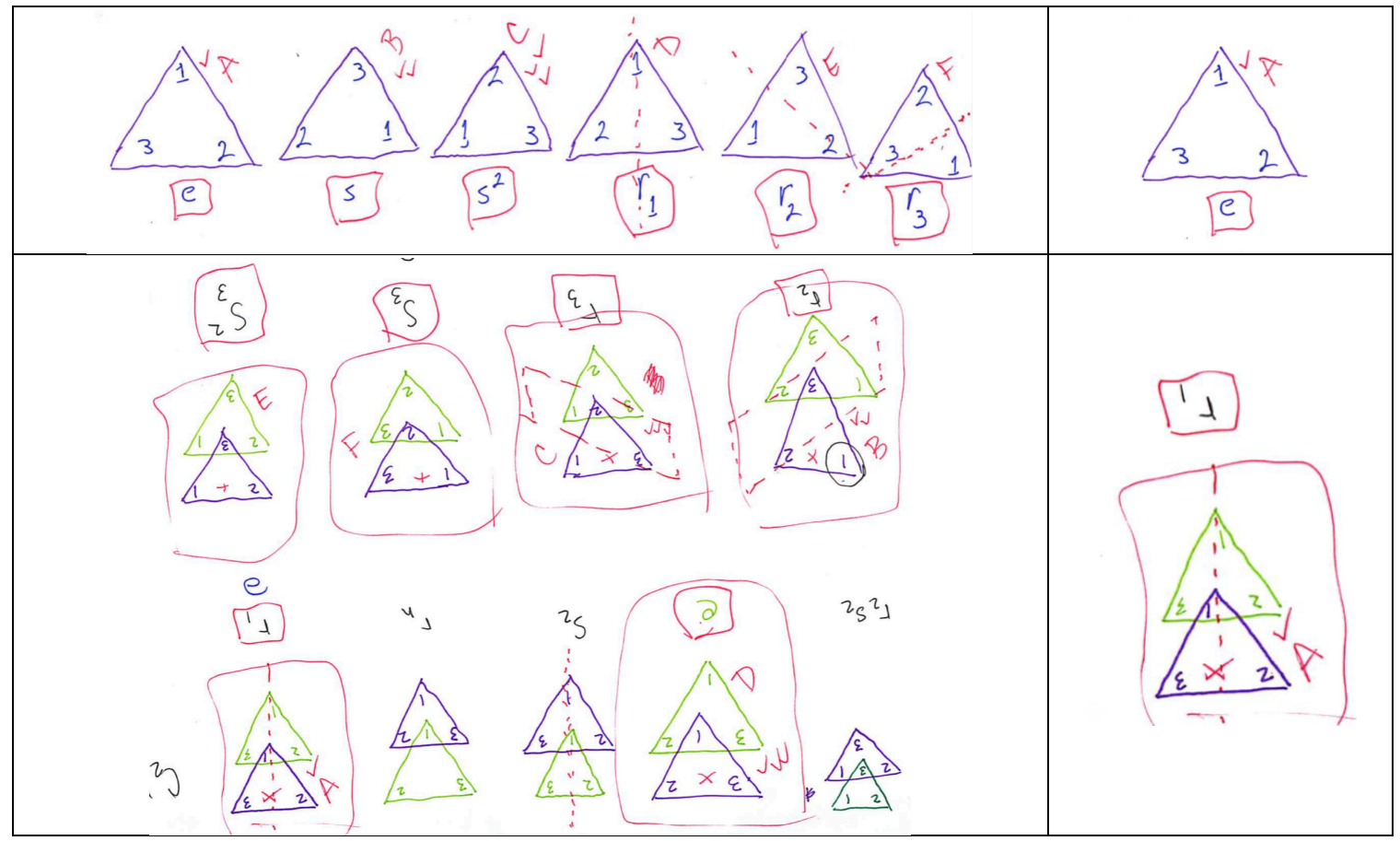

Figure 65. Arthur and Stu's initial identification of six elements in ethane to build a correspondence with $\mathrm{D}_{6}$.

This approach of associating the symmetries with vertex configurations was very limiting for Arthur and Stu and they continued to struggle arguing that their 
correspondence was structure preserving, and for myself in terms of being able to make sense of their thinking. More specifically, it makes it difficult to know which objects they are actually thinking of at any given point in time the configuration of the figure, the permutation of the labels of the vertices, or the symmetry transformation. For example, while the "upside down" version of $D_{6}$ seen in Figure above, isn't isomorphic to $D_{6}$ if it represents a subset of the twelve symmetries, but the permutation of the vertices is isomorphic to $D_{6}$. So, while they ultimately did make a Cayley table out of the six elements of ethane they had corresponded with ammonia, using the map in Figure 66 below, their reflections remained twisted and an isomorphism continued to elude them.

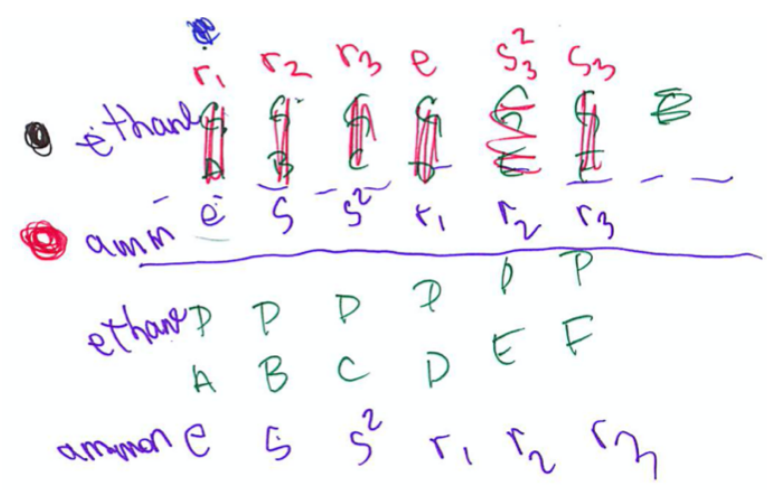

Figure 66. Arthur and Stu's explicit correspondence between six symmetries of ethane and ammonia.

Arthur and Stu's approach to this task suggests that by the time students start to argue whether two symmetry groups are isomorphic, they will want to be committed to treating the symmetries as transformations. By the time they take on isomorphisms, states should be seen as merely part of a record keeping system for analyzing what happens when certain transformations are combined; and a record keeping system they are becoming increasing less dependent on. Their inability to establish an isomorphism between the sets also showed that Arthur and Stu's ideas about isomorphism weren't quite robust enough yet to support them in being sure that they were looking at a group isomorphic to 
$D_{6} \times \mathbb{Z}_{2}$. This is another instance where knowing that orders of elements are preserved would have been helpful.

Could this be a Cayley table for ammonia?

\begin{tabular}{|l|l|l|l|l|l|l|}
\hline$*$ & $A$ & $B$ & $C$ & $D$ & $E$ & $G$ \\
\hline$A$ & $B$ & $A$ & $D$ & $C$ & $G$ & $E$ \\
\hline$B$ & $A$ & $B$ & $C$ & $D$ & $E$ & $G$ \\
\hline$C$ & $G$ & $C$ & $B$ & $E$ & $D$ & $A$ \\
\hline$D$ & $E$ & $D$ & $A$ & $G$ & $C$ & $B$ \\
\hline$E$ & $D$ & $E$ & $G$ & $A$ & $B$ & $C$ \\
\hline$G$ & $C$ & $G$ & $E$ & $B$ & $A$ & $D$ \\
\hline
\end{tabular}

Figure 67. Supplemental task to support Arthur and Stu's thinking about isomorphisms 2

In reaction to Arthur and Stu's dependence on associating symmetries with states instead of transformations I decided to present them with a task that would allow me to focus on their thinking about isomorphisms without the messiness of tangled rotations. I chose to use "the mystery table" (see Figure 67 above) adapted from the IOAA curriculum (Larsen, 2010a) as it has been shown elicit informal student thinking around this very concept (Larsen, 2009). Stu says, "superficially it looks like no, because there isn't a perfect match for the way they have it written, but that doesn't necessarily mean anything" and they dig in. They start by quickly identifying that element B must be the identity and then start checking the order of the remaining elements. Stu begins to superficially match up elements in the mystery table with elements in ammonia creating a bijection the bijection seen in Figure 68 below. 


$$
\begin{aligned}
& f=\left(\begin{array}{llllll}
A & B & C & D & E & G \\
r_{1} & e & r_{2} & S & r_{3} & S^{2}
\end{array}\right) \\
& f=\left(\begin{array}{llllll}
B & A & C & E & D & G \\
e & r_{1} & r_{2} & r_{3} & S & S^{2}
\end{array}\right)
\end{aligned}
$$

Figure 68. Arthur and Stu's element-wise mapping from the mystery table to ammonia; version 1

Once Stu completes his bijection Arthur tentatively asks:

Arthur: So should we fill in one of these (a Cayley table) that has been provided for us?

Annie: You can, you don't have to, whatever helps.

Arthur: Ok, so...

Stu: So last time we tried to get like, take one and get the other, right? Can we do something like that again? Can we put B here (starts to fill in the beginning of a blank Cayley table seen in Figure 69). And then what we think is $r_{1}$ which would be A, or what our first guess of $r_{1}$, is? See what I'm saying? Does this make any sense to do or does that help us at all?

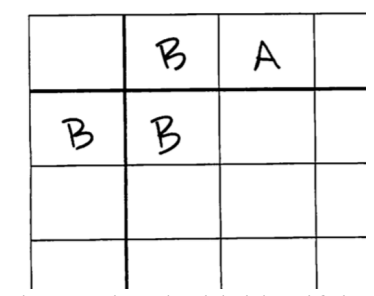

Figure 69. Stu's written work associated with identifying the mystery table as $\mathrm{D}_{6}$

Arthur: I'm super apprehensive about, about linking any, I think you're thinking let's just fill in an order and see if it works and then we'll find it right? Ok, I think I'm letting myself get brickwalled, because if we like, if this A 
(points to new Cayley table) is the wrong, has us thinking about the wrong $r$ (in ammonia) that kinda cascades through.

Stu: Ok, I've got another idea.

Arthur: Yeah.

Stu: What if we combine the $r^{\prime}$ s and the $s$ 's over there (ammonia) then can we deduce which is which?

Arthur and Stu proceed to check pairs of reflections in ammonia and discover some of them have contradictory outputs when compared to the mystery table using their original mapping. Using these findings, they redefine their mapping once more so that the result of a combination in ammonia matches "the same" combination in the mystery table as seen in Figure 70 below.

$$
\begin{aligned}
& f=\left(\begin{array}{llllll}
A & B & C & D & E & G \\
r_{1} & e & r_{2} & S & r_{3} & S^{2}
\end{array}\right) \\
& f=\left(\begin{array}{llllll}
B & A & \& & C & D & G \\
e & r_{1} & r_{2} & r_{3} & S & S^{2}
\end{array}\right)
\end{aligned}
$$

Figure 70. Arthur and Stu's element-wise mapping from the mystery table to ammonia; version 2

Armed with their map, Stu suggests a new attack plan. He suggests they 1) use their map to fill in the headers of a new Cayley table, 2) use the mystery table to fill it out the body. Once the table is complete, they can 3) use it to see if this new table matches their Cayley table for ammonia; Arthur agrees with Stu's strategy for determining isomorphism. Stu grabs a blank Cayley table and fills out the header with an element from both ammonia and the mystery table, paired up by their map (see Figure 71 below). 
Then, following their own directions, Stu fills out the body of the Cayley table first using only elements from the mystery table (leaving room in each box) seen in part a of Figure 71 below. Then Stu goes back and fills in the table again using their map in Figure 70 above to rename each element as a symmetry. Meanwhile Arthur is checking that everything corresponds with their Cayley table for ammonia, seen in part b of Figure 71. As soon as Arthur shad finished scanning each table row by row checking for equivalence, in the excitement of the moment before they had a chance to explain their conclusion, I quickly exclaimed that I had given them a table for $D_{6}$ and that they had successfully argued that it was isomorphic. I went on to remind them what isomorphisms are, and I told them how they did everything they needed to show that their groups were isomorphic unfortunately taking their opportunity to explain their findings to me.

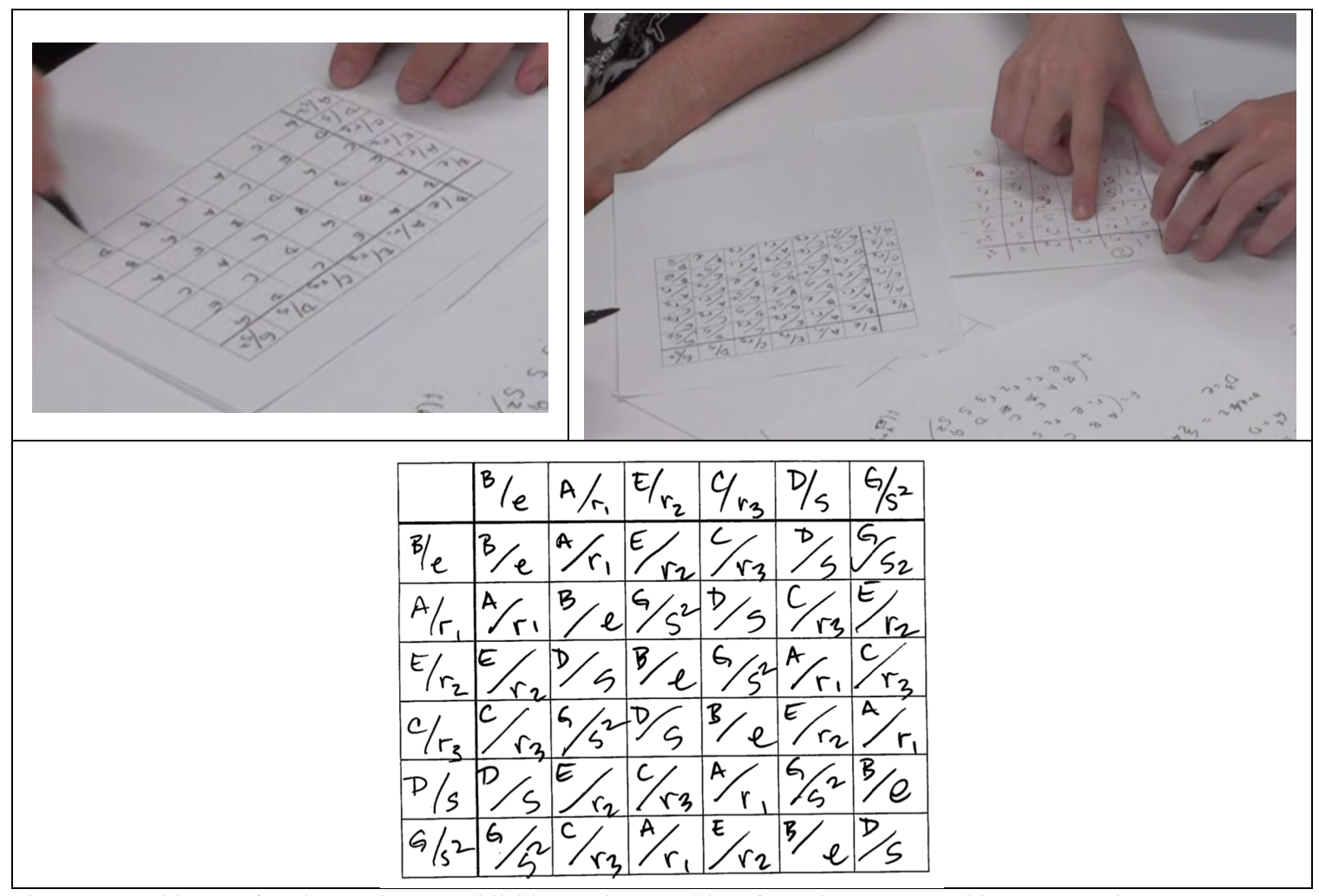

Figure 71. Evidence of Arthur and Stu establishing an isomorphism from the mystery table to ammonia. 
This task proved extremely useful for gaining a clearer understanding of Arthur and Stu's thinking about isomorphisms in general. It showed that they had a good understanding of the necessary requirements for group equivalence and it evoked a strategy for establishing such a relation, even though I the excited researcher failed to give them an opportunity to explain it all together at once. This task demonstrated that at least if given more abstract elements, Arthur and Stu had all the right components to successfully establish isomorphisms between groups. One possible follow up task to this in the future would be to have them prove that "Mystery Table $\mathrm{x} \mathbb{Z}_{2}$ " is isomorphic to ethane. Instead, I decided to have them produce new, different representations for $D_{6} \mathrm{X}$ $\mathbb{Z}_{2}$ before returning to arguing equivalence.

a. Describe the elements of $D_{6} \times \mathbb{Z}_{2}$ as if we were in a group theory course. What would the group look like in a textbook? Come up with a representation for $D_{6} \times \mathbb{Z}_{2}$ using any elements that you want.

b. Prove that your symmetry group for ethane is isomorphic to your more conventional representation of $D_{6} \times \mathbb{Z}_{2}$.

Figure 72. Supplemental task to support Arthur and Stu's thinking about isomorphisms 3

Now that it was clear Arthur and Stu at least understood the necessary components for establishing a group isomorphism, we decided to return our focus to the group of symmetries for ethane. Again, the mystery table task showed that if given more abstract elements, Arthur and Stu could correctly establish isomorphisms between groups and so I wanted them to create a version of $D_{6} \mathrm{x} \mathbb{Z}_{2}$ so that the elements in the set appeared more like the abstract sets they would have had experience with in their group theory course. The prompt used, in Figure 72 above, also helped to gain further insight into the students thinking of external direct products as ordered pairs. When asked to describe the 
elements of $D_{6} \times \mathbb{Z}_{2}$ using whatever they might describe as 'conventional notation' Arthur and Stu both quickly responded with very similar ideas. Arthur described his thinking in the following excerpt:

Annie: Can you come up with a representation for $D_{6} \times \mathbb{Z}_{2}$ for me? You can use any elements you want.

Arthur: I like ordered pairs for that. I mean it could be even ordered pairs of like the symmetries in $D_{6}$ and the other coordinate is just a 1 or a 0 . I think that's a more logical way to think about it, whereas when we started filling in our table, the like color swap elements had their own. So it's like we had 12 unique group elements instead of 12 like you cognitively unique elements, instead of 6 elements and a flipped version of each one. So I lean toward ordered pairs of 1 or 0 for each $D_{6}$ element. Even though I know that we could do it as all the unique symmetries of our ethane because we did that, and it was a headache.

Stu. Yeah. If I was doing a homework assignment, I would write it like (see Figure 73 below). 


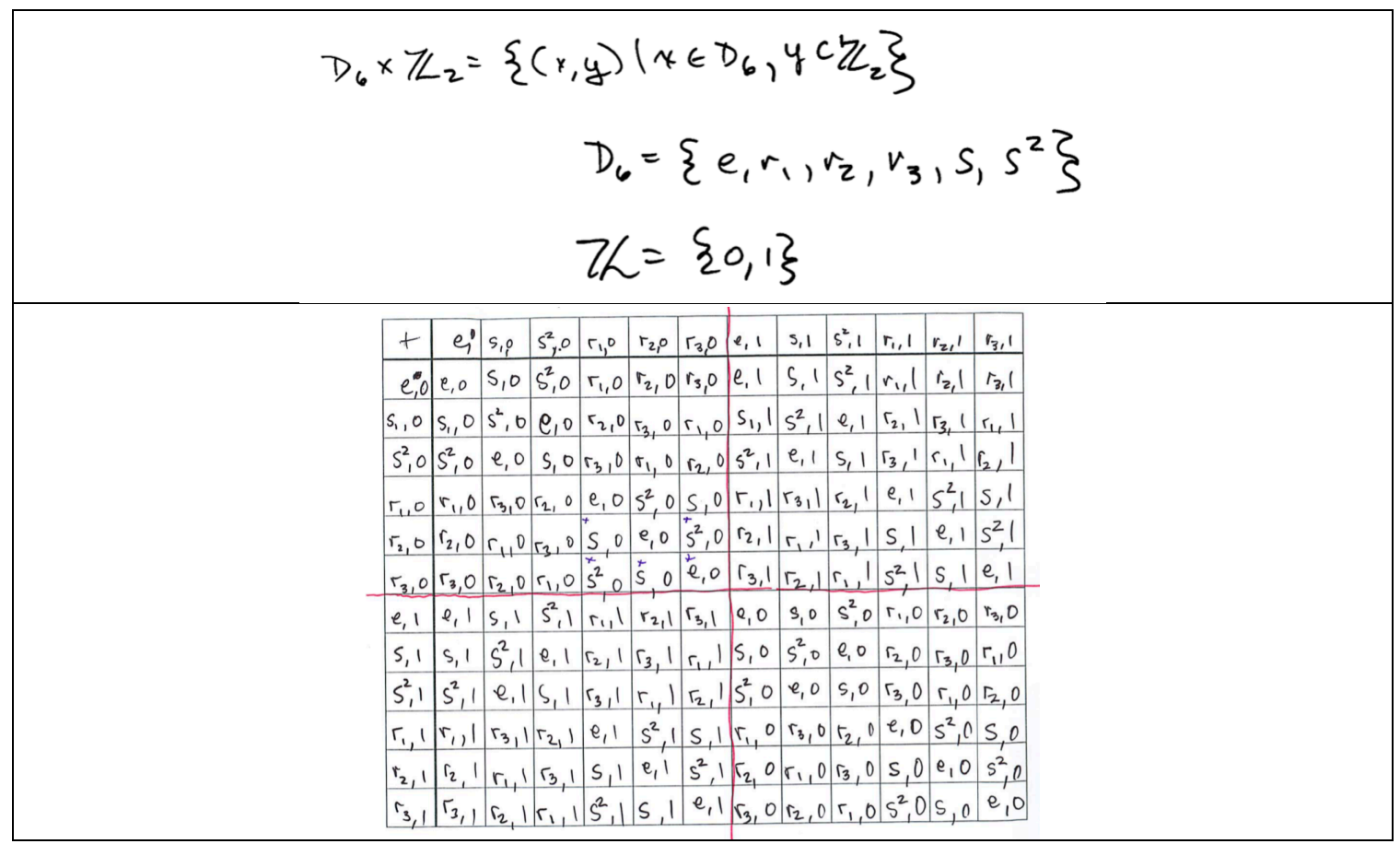

Figure 73. Stu's set notation description of $\mathrm{D}_{6} \times \mathbb{Z}_{2}$ and accompanying Cayley table.

Stu started by creating a bijection between their new conventional representations of $D_{6} \times \mathbb{Z}_{2}$ and their elements of ethane, seen in Figure 74.

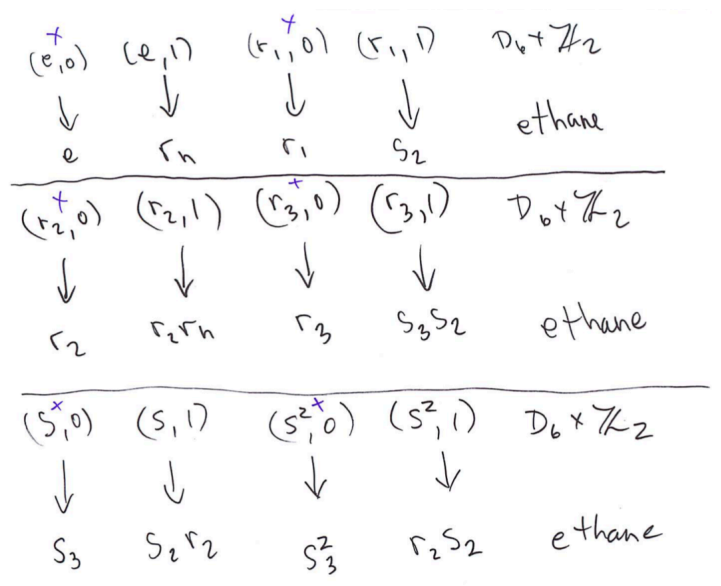

Figure 74. Stu's bijection from $\mathrm{D}_{6} \times \mathbb{Z}_{2}$ to ethane.

However, due to how their elements in ethane were labeled from ground up observations as Stu previously mentioned, they really struggled to both fill in the Cayley table, especially products involving the element $s_{2}$ as seen in Figure 75 below. The students spent a lot of time trying to determine combinations of symmetries, but 
ultimately Arthur and Stu found that they needed to use the physical molecule for each combination. While Arthur manned the molecule, recording the resulting orientation after series of symmetries, Stu filled in the Cayley table. Some of Arthur's recordings can be found in Figure 75 below.

\begin{tabular}{|c|c|c|c|c|c|c|c|c|c|c|c|c|c|c|c|}
\hline * & $e$ & $S_{3}$ & $5_{s}^{2}$ & $r_{1}$ & $r_{2}$ & $r_{3}$ & $r_{h}$ & $s_{2} r_{2}$ & $r_{2} s_{2}$ & $S_{2}$ & $r_{2} r_{h}$ & $S_{3} s_{2}$ & 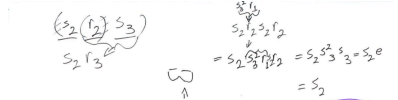 & \multirow{2}{*}{\multicolumn{2}{|c|}{ 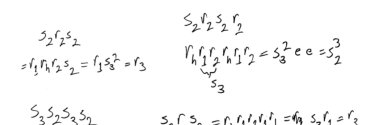 }} \\
\hline$e$ & $e$ & $\mathrm{~S}_{3}$ & $s_{3}^{2}$ & $r_{1}$ & $r_{2}$ & $r_{3}$ & $r_{h}$ & $S_{2} r_{2}$ & $r_{2} s_{2}$ & $S_{2}$ & $r_{2} r_{h}$ & $S_{3} s_{2}$ & \multirow{5}{*}{ 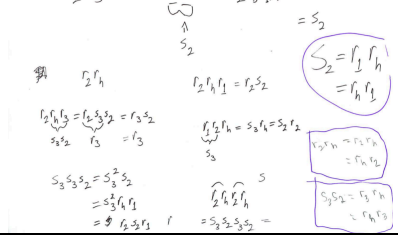 } & & \\
\hline$S_{3}$ & $s_{3}$ & $s_{3}^{2}$ & $e$ & $4_{4}{ }^{1 / 3}$ & $f_{1}$ & $r_{2}$ & $s_{2} r_{2}$ & & & & & & & \multirow{4}{*}{$\begin{array}{l}S_{3} r_{1} r_{h} s_{3} r_{1} r_{h} \\
=S_{3} r_{1} S_{3} r_{1} r^{r} h \\
=r_{3} r_{3} r_{h} r_{h}=e\end{array}$} & \multirow{4}{*}{$\begin{array}{l}s_{2} r_{2} r_{2} r_{h} \quad s_{2} r_{h}=8 r_{1} \\
s_{2} r_{3} s_{3} s_{2}=r_{1} r_{2} s_{3} r_{1} \\
=s_{3}^{2} s_{1}=r_{2} \\
r_{2} s_{2} r_{2} s_{2}=r_{2} r_{2} r_{2} r_{1}=s_{3}^{2} s_{3}^{2}=s_{3} \\
r_{2} s_{2} s_{2}=r_{2} \quad r_{2} s_{2} s_{2} r_{h}=s_{2} r_{1} r_{2}=s_{3}^{2} r_{2}=r_{3}\end{array}$} \\
\hline$S_{3}^{2}$ & $S_{3}^{2}$ & $e$ & $\mathrm{~S}_{3}$ & $r_{2}$ & $r_{3}$ & $r_{1}$ & $r_{2} s_{2}$ & & & & & & & & \\
\hline$r_{1}$ & $r_{1}$ & $r_{2}$ & $r_{3}$ & e & $s_{3}$ & $S_{3}^{2}$ & $S_{2}$ & & & & $S_{2} r_{2}$ & & & & \\
\hline$r_{2}$ & $r_{2}$ & $r_{3}$ & $r_{1}$ & ${ }^{+} S_{3}^{2}$ & $e$ & ${ }^{\top} \delta_{3}$ & $r_{2} r_{h}$ & & & & $f_{h}$ & & & & \\
\hline$r_{3}$ & $r_{3}$ & $r_{1}$ & $r_{2}$ & ${ }^{*} \mathrm{~S}_{3}$ & $S_{3}^{2}$ & $e$ & $S_{3} S_{2}$ & & & & & & \multirow{8}{*}{$\begin{array}{ll}r_{2} s_{2} s_{3} s_{2}=r_{2} r_{1} s_{3} r_{1}=s_{3}^{2} s_{3} r_{1}=r_{1} & s_{2} s_{3} s_{2}=r_{1} s_{3} r_{1}=r_{2} r_{1}=s_{3}^{2} \\
s_{2} r_{2} r_{h}=r_{1} r_{2}=s_{3} & r_{2} s_{2} r_{2}=r_{2} r_{1} r_{1}=s_{3}^{2} r_{2}=r_{3} \\
r_{2} r_{2} r_{2} s_{2}=r_{2} r_{2} r_{1}=r_{1} & r_{2} r_{h} s_{3} s_{2}=r_{2} s_{3} r_{1}=r_{3} r_{1}=s_{3} \\
s_{3} s_{2} s_{2} r_{2}=s_{3} r_{2}=r_{1} & s_{3} s_{2} r_{2} s_{2}=s_{3} r_{2} r_{2} r_{1}=r_{3} r_{2} r_{1}=s_{3}^{2} r_{1}=r_{1} \\
s_{3} s_{2} r_{2} r_{h}=s_{3} r_{1}=r_{3} r_{2}=s_{3}^{2} & \end{array}$} & \multirow{8}{*}{\multicolumn{2}{|c|}{ 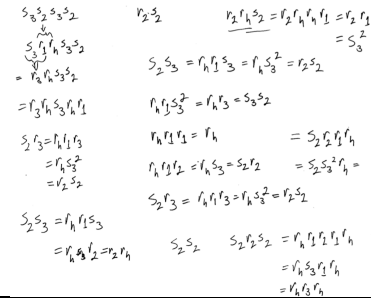 }} \\
\hline$r_{h}$ & $r_{n}$ & $s_{2} r_{2}$ & $r_{2} s_{2}$ & $S_{2}$ & $r_{2} r_{n}$ & $S_{3} S_{2}$ & $e$ & $S_{3}$ & $S_{3}^{2}$ & $r_{1}$ & $r_{2}$ & $r_{3}$ & & & \\
\hline $\mathrm{S}_{2} \mathrm{r}_{2}$ & $s_{2} r_{2}$ & & & & & & $S_{3}$ & ${ }^{2} \mathrm{~S}_{3}^{2}$ & e & $r_{3}$ & $r_{1}$ & $r_{2}$ & & & \\
\hline$r_{2} s_{2}$ & $r_{2} s_{2}$ & & & & & & $S_{3}^{2}$ & e & $s_{3}$ & $r_{2}$ & $r_{3}$ & $r_{1}$ & & & \\
\hline $\mathrm{S}_{2}$ & $s_{2}$ & $r_{2} \mathrm{rn}_{\mathrm{n}}$ & $\mathrm{S}_{3} \mathrm{~S}_{2}$ & $r_{n}$ & $S_{2}{ }^{2}$ & $r_{2^{2} n}$ & $r_{1}$ & $r_{2}$ & $r_{3}$ & $e$ & $s_{3}$ & $s_{3}^{2}$ & & & \\
\hline$r_{2} r_{h}$ & $r_{2} r_{h}$ & & & $r_{2} S_{2}$ & & & $r_{2}$ & $r_{3}$ & $r_{1}$ & $S_{3}^{2}$ & e & $s_{3}$ & & & \\
\hline $\mathrm{S}_{3} \mathrm{~S}_{2}$ & $\mathrm{~S}_{3}^{5} 2$ & & & & & & $r_{3}$ & $r_{1}$ & $r_{2}$ & $S_{3}$ & $S_{3}{ }^{2}$ & $e$ & & & \\
\hline & & & & & & & & & & & & & & & \\
\hline
\end{tabular}

Figure 75. A sample of Arthur and Stu's written work associated with trying to establish an isomorphism.

Overall, this task had mixed success. While it was very useful for the students to have a more conventional representation of the group $D_{6} \times \mathbb{Z}_{2}$, ultimately the task took a very long time and was very frustrating to them. Their original naming of the symmetries of ethane did not make it obvious which elements were the combination of an element they were considering " $D_{6}$ with a color swap." During ongoing analysis after the session it became clear that I needed another prompt in order to help them find a way out of the confusion and make the elements in ethane more obvious and easier to work with.

Rename (the second half, recognizing that some may already be nice) your symmetries of ethane so that they capture your observation that they are really just purple elements with a color swap.

Figure 76. Supplemental task to support Arthur and Stu's thinking about isomorphisms 4 
Eventually realizing there might be a way to help them out of their labeling nightmares, but again unaware of the transposition of their tables, I asked the students to re-write all their elements in ethane to better reflect the binary information they were capturing in their conventional $D_{6} \times \mathbb{Z}_{2}$ element descriptions. This task was intended to help the students leverage the fact that the combinations of symmetries that seemed to be easiest to compute were the ones that had an $r_{h}$ in them. Such a renaming would also move students closer towards a more conventional representation of an inner direct product where each element is a product of elements from different commutative subgroups with a trivial intersection. The students quickly renamed the elements in ethane to either have an $r_{h}$ or not, similar to their direct product having a second component of 0 or 1 as seen in Figure 77.

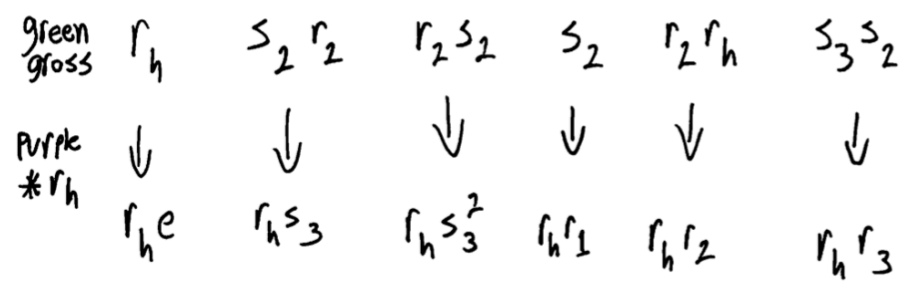

Figure 77. Arthur and Stu's renaming of elements of ethane to better represent the direct product structure.

Using the new elements names Arthur and Stu were able to quickly make a new Cayley table, seen in Figure 78 below. 

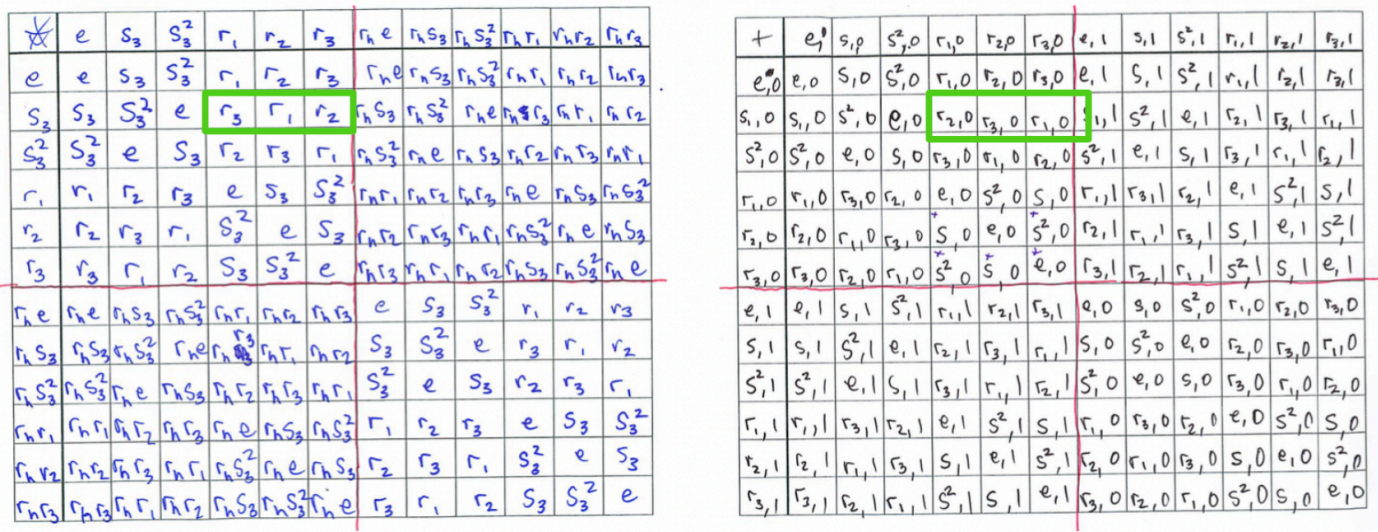

Figure 78. Arthur and Stu's Cayley tables for the symmetries of ethane (left) and $\mathrm{D}_{6} \times \mathbb{Z}_{2}$

This proved to be an extremely useful task and it definitely helped Arthur and Stu move closer towards an isomorphism. However there are still ways this task can be improved. First it suggests a design choice, I could ask the students to explicitly rename their elements to highlight the color swap, as I did. Alternatively I could have asked the students if they can look at their naming and "see" something that looks like what they listed for $D_{6} \times \mathbb{Z}_{2}$. This might have allowed the Stu to use this thinking about direct products more explicitly since at this point he had already demonstrated some understanding of which symmetry was necessary for the direct product, specifically the reflection he referred to as "independent."

Unfortunately this task also had a serious drawback, it only attended to the superficial differences in the representations of the elements. While again it was powerful for the students to rename the symmetries of ethane so that they "looked" more like the elements of $D_{6} \mathrm{x} \mathbb{Z}_{2}$ it is also important the correspondences are made between elements that behave the same instead of just look the same. This unfortunately was the case for Arthur and Stu. The renaming didn't make the isomorphism as obvious as I had intended, and they continued to struggle with establishing an isomorphism because while it make the direct product behavior more obvious the real issued ended up being in their labeling of 
$D_{6}$. However, we were all unaware of the underlying labeling issue and so we continued to try to establish an isomorphism.

Armed with a complete Cayley table for their symmetries of ethane, and a Cayley table for the group $D_{6} \times \mathbb{Z}_{2}$ Arthur and Stu were ready to argue that they were isomorphic. We began by my asking them the following:

Annie: Now how would you argue that that (their newly created Cayley table for ethane) is isomorphic to this (their Cayley table for $D_{6} \times \mathbb{Z}_{2}$ )?

Stu: (to Arthur) I'll let you talk first.

Arthur: Remind me the formal requirements for isomorphism...

Annie: So we said that it has to be a structure preserving bijection.

Arthur: Ok. It's a bijection, one element goes to one element ... and... I don't know how can we say it's structure preserving... like it... there are lots of little substructures we can point out, but I could use a refresher on how we argue structure preservation as a whole.

Annie: So Stu, wrote it down, hold on one second... so when you guys were doing the mystery table...

Arthur: I feel like we need to show that kind of... (Arthur writes down the homomorphism property as an equation, $f(x y)=f(x) f(y))$

Annie: Yeah, so when we were doing the mystery table, yeah you wrote that over here as well.

Arthur: Ah, I like that. 
While Arthur and Stu were able to successfully argue an isomorphism between the mystery table and their group for ammonia, it seemed to be less clear to them how to make a similar argument between ethane and $D_{6} \times \mathbb{Z}_{2}$. I remind them that they checked combinations of symmetries in each table and check to see if the result was consistent with their mapping and so they turned to start checking similarities between their tables. Unfortunately, shortly into checking, Stu noticed inconsistencies amongst the reflections (seen with green boxes in Figure 78 above). He points out that their reflections are again "twisted" saying, "there's like an ordering issue, and if we can... we could probably sort the ordering issue, like $r_{1}$ doesn't go to $\left(r_{1}, 0\right)$, but it probably goes to $\left(r_{2}, 0\right)$ or $\left(r_{3}, 0\right)$ and we can sort that out and say, ok good to go. My hope." He goes on to point out that this twisting issue is going to repeat itself throughout each of the quadrants of the Cayley table.

In an effort to "untwist" their reflections Stu focuses on the reflections in the first quadrant and produces a new map seen in Figure 79.

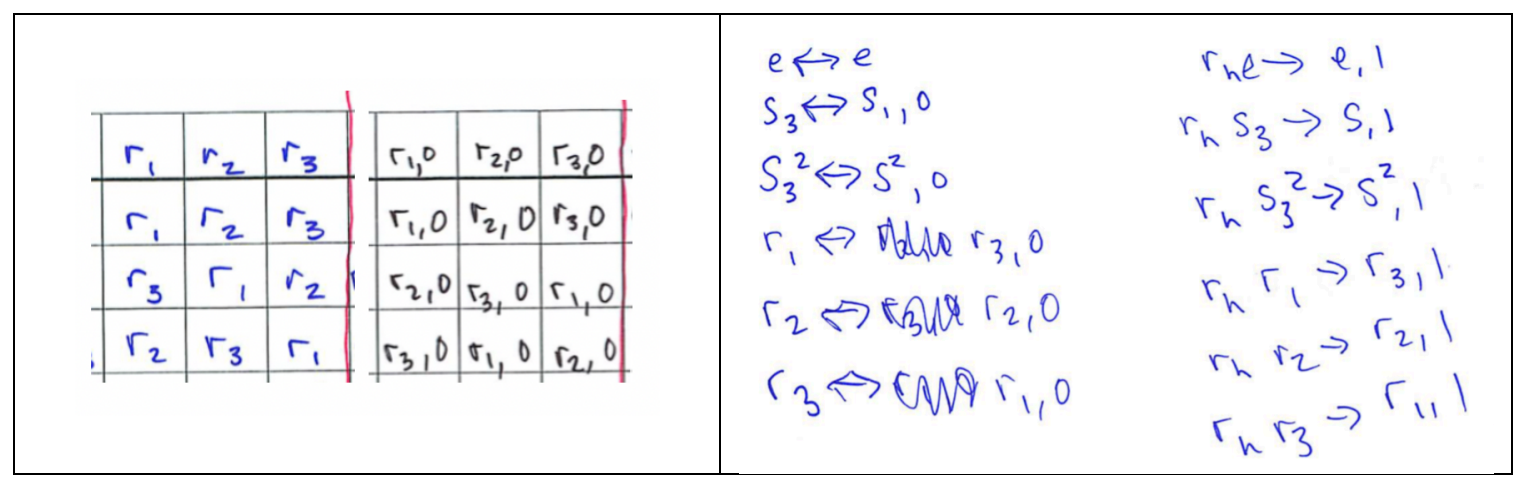

Figure 79. Stu's written work associated with trying to untangle an isomorphism

Unfortunately, this mapping still produces discrepancies when they go to check outcomes between the two tables. Finally I break down and the following transpires: 
Arthur: I'm lost.

Stu: Yeah, I thought I had it.

Annie: Can you give me an idea of what we're trying to do? If you can convince me that you know what you're try to get, like what are you looking for and what's it gonna tell you?

Stu: I'm trying to swap the columns so that it's easy to match these (the reflections in one groups) with these (the reflections in the other group).

Annie: What would that tell you?

Stu: It would tell me that the structure is intact between the two. That the bijection holds the same algebraic structure in both Cayley tables.

From there I decide to move on to a new molecule, leaving them convinced they are the same groups, but admittedly unsatisfied, because we never actual figured out a correct mapping. In hindsight, this was a somewhat unfortunate decision that I made, but in the moment, it felt necessary and justified. At this point in both the session and the TE overall, we had already spent a considerable amount of time trying to establish the isomorphism, and I still had LIT related tasks that I needed to turn my attention to in order to inform the further development of the LIT.

Long after the completion of the teaching experiment it finally became clear why Arthur and Stu continued to struggle to establish an isomorphism. In retrospective analysis I realized that it was the Cayley table Arthur and Stu had created for $D_{6} \mathrm{X} \mathbb{Z}_{2}$ with "conventional representations" that was causing them such grief. For every other Cayley table that Arthur and Stu created during the teaching experiment they established 
the convention of combining elements row first followed by column elements. Deciding which element is combined first, row or column, is especially important in groups that are non-commutative because while there does exist an isomorphism between a Cayley table and its transpose, it is non-trivial and not obvious. For whatever reason Arthur and Stu created their Cayley table for $D_{6} \mathrm{x} \mathbb{Z}_{2}$ by combing columns elements followed by row elements. Therefore labeling was always against them.

In hindsight it would have been more productive to structure this task a bit differently. First, I could have had them re-write their symmetry elements of ethane so that they look exactly like an internal direct product version of $D_{6} \mathrm{x} \mathbb{Z}_{2}$ leveraging the fact that all the elements can be represented as a product of two subgroups. Then I could have students compare this new version of the symmetries of ethane to their more conventional version of $D_{6} \times \mathbb{Z}_{2}$. In order for this to work, the symmetry elements in ethane would need to look like standard elements of $D_{6}$, (by standard I mean so that they look more obviously like an inner semi direct product) $\left\{\mathrm{I}, \mathrm{R}, \mathrm{R}^{2}, \sigma_{\mathrm{v}}, \sigma_{\mathrm{v}} \mathrm{R}, \sigma_{\mathrm{v}} \mathrm{R}^{2}\right\}$ times something in their $\mathbb{Z}_{2}$ subgroup of ethane, the other reflection $\sigma_{\mathrm{h}}, \sigma_{\mathrm{v}} R \sigma_{\mathrm{h}}$. This alternative approach should make the mapping between the two groups more obvious, or at least visible enough for them to see similarities between transformation beyond superficial labels that don't describe the motion such as their $r_{1}, r_{2}$, etc. In fact there already exists such a task in the IOAA curriculum that supports students in generating this kind of symbol set, see Larsen $2010 \mathrm{~b}$ for the task and rationale.

\footnotetext{
a. If I just handed you another molecule could you tell if it was going to be a $D$-something $\mathrm{x} \mathbb{Z}_{2}$ ? What would you look for?

b. Describe something that you would expect to have a $D$-something $\mathrm{x} \mathbb{Z}_{2}$ symmetry group.
} 
Figure 80. Supplemental task to support Arthur and Stu's thinking about isomorphisms 6

In a final attempt to gain deeper insight on Arthur and Stu's thinking about directproducts I asked them a final prompt, found in Figure 80 above. The goals for this task was to test the students' familiarity with identifying groups that are isomorphic to external direct products, in other words, to see if the students have really gained ownership over recognition of the particular group structure.

After asking the prompt Arthur immediately replies:

Arthur: I'd look for that regular polygon. I would look to be able to see it, how do I say this... looking for regular polygon, I feel good about that part of the process, and then I guess saying like how many nested symmetries now, because I can look at this square and there's another square that I can look at that's indistinguishable from the first. So that's kind of our $\mathbb{Z}$ category, our number of color options. But yes, look for the polygon and then how many different ways can I do it, can I see that same polygon indistinguishable from the first. And I probably couldn't say anything better than that.

Stu: I think that other thing is that we don't want... with both of these molecules (ethane and cyclobutene) we have a horizontal reflection, but it's not, we don't have... if this (cyclobutene) was a cube that would be a problem, that's what I'm trying to say. 
Stu goes on to explains that a cube would make this much more confusing by introducing a bunch of new symmetries. I remind them that the platonic solids are really their own beast and eventually we return to our conversation:

Annie: Could you maybe describe a shape of something that you expect to be a $D$-something x $\mathbb{Z}_{2}$ ?

Arthur: Just crack this open (a bond in cyclobutene) and put another one of these (group of atoms) so that we just had a regular pentagon, yeah then we would have $D_{10} \times \mathbb{Z}_{2}$.

Annie: So when we find these things that are kind of like a d-group cross a $\mathbb{Z}_{2}$, the $D$-group is coming from the polygon? Is that right?

Arthur: Umm hmm.

Stu: Umm hmm.

Annie: So we see some kind of polygon?

Arthur: Umm hmm.

Stu: $\quad U m m ~ h m m$.

Annie: ....and then the $\mathbb{Z}_{2}$, sorry one more time...

Stu: Is coming from this plane of reflection, between the two polygons. And it's not really the molecules it's the plane of reflection.

This is evidence that Arthur isn't seeing the difference between what happens when you flip (180-degree rotation) and when you reflect. This is significant in that Arthur is describing a semi-direct product which only gives the dihedral part of the group. It is 
also kind of an interesting description because in a conventional classification system in chemistry they often associate dihedral groups with the existence of a second rotation orthogonal to the rotational axis instead of a horizontal reflection. In that sense, one might say, Arthur is thinking more like a chemist and so ultimately he could produce an entirely different flowchart, asking about different symmetry combinations.

Summary of Case 1: Arthur and Stu

Stu's description of the symmetry group of ethane as "like ammonia cross $\mathbb{Z}_{2}$ " ended up being an extremely powerful conceptualization that I used to help support Arthur and Stu in taking more ownership over the use of standard group names and thus successfully complete Step 2 of the LIT. Overall there were a number of lessons learned about how to adapt the local instructional theory into a series of supplemental tasks in order to support Arthur and Stu. The first was that I need to really understand my students' notation so that I can best understand their thinking, so that I could see where exactly to focus the additional support. Stu's initial description of the symmetries of ethane as "like ammonia cross $\mathbb{Z}_{2}$ " suggested that perhaps a conversation about direct products would help them in being more confident with the use of standard group names. Then after providing some prompts aimed to get a deeper understanding of how Arthur and Stu were thinking about direct products it became clear that they didn't have enough language to confidently say their group was isomorphic to $D_{6} \times \mathbb{Z}_{2}$, and so the focus then shifted to isomorphisms.

Even though Stu had presumably all the fundamental ideas about direct products, and seemed to at least implicitly recognize commutativity as important, his overall description of direct products remained vague enough to be more of a metaphorical 
understanding. Together their activity was something along the lines of, they had a sense of what a direct product was... and this group was kind of like that. Unfortunately, while it would have been extremely interesting to try and move them towards reinventing internal direct products, it seemed unlikely and so we decided instead to support them in trying to prove that their symmetry group was isomorphic to the external direct product they claimed it to be.

Then in reaction to Arthur and Stu's activity, I focused on supporting Arthur and Stu's understanding of isomorphism so that they could more comfortably use standard group names to describe groups. To do so I created a series of six supplemental tasks which were successful to a varying degree, as described above. In retrospect I think that the sequence of tasks in their entirely had a lot of potential and probably could have gone a bit better than they did. Unfortunately, Arthur and Stu struggled with committing to thinking about symmetries as transformations as opposed to orientations throughout the entirety of the teaching experiment. While I had explicit conversations with them about how they really needed to be thinking about symmetries as transformations in order to do group theory, they still continued to use states. This led to an overdependence and concern with labeling and matching labels instead of matching behaviors of transformations which ultimately made establishing isomorphisms extremely difficult.

Overall Case 1 can be considered a partial success as there are important lessons that were learned. For instance Arthur and Stu's choice of symbols was confusing from the very beginning. The results of Case 1 along with the successes described below in Case 2 give strong evidence for motiving, if not requiring students to represent their symmetry elements in terms of generators. Arthur and Stu's use of opaque symbols along with this 
dependence on their symmetry configurations shows just how important it is to have students try to use as clear and understandable notation as possible. I suspect that had Arthur and Stu been encouraged to rename their symmetry elements for ethane in terms of the three generating symmetries instead of just in terms of $D_{6}$ and the horizontal reflect $r_{h}$ constructing isomorphisms would have been much more obvious. Lastly, had I the researcher noticed that the Cayley table they created for $D_{6} \times \mathbb{Z}_{2}$ which they were continually attempting to argue an isomorphism with, was a transpose of what they had intended it undoubtably would had led to less tangled experience.

\section{Case 2: Ada and Sophie}

In teaching experiment 3, TE3, Ada and Sophie had a very different approach from Arthur and Stu. Instead of trying to describe each of their collections of symmetries using convention group names Ada and Sophie described every one of their groups using group presentations. This choice of representations was the direct result of their supplemental group theory education. In order to introduce Ada and Sophie to the notion of a group, they were provided with the first IOAA module which consists of a LIT for the guided student reinvention of the group concept through an in-depth exploration of the symmetries of a regular polygon. Due to the nature of IOAA, students are led to describe the symmetry group of their polygon using a group presentation. It is only the instructor who could provide the idea that this structure has a conventional name, and again Ada and Sophie had no prior experience with group theory, so conventional group names were unavailable to them. 
The presentation of a group, or group presentation is a method of representing a group by listing a set of generating elements, where every other element in the group can be written as a product of the generators, and relations amongst the generators. Ada and Sophie's group presentations for the symmetry groups of water, ammonia, and ethane can be seen in Figure 81 below as examples.

\begin{tabular}{|c|c|c|c|}
\hline $\begin{array}{ll}O F=F & \text { Associative } \\
O R=R \quad & F R=R F \\
2 R=O & \text { Inverses } \\
2 F=O & \end{array}$ & $\begin{array}{l}R^{3}=0 \quad F^{2}=0 \\
O R=R \quad O F=F \\
R F=F R^{3}\end{array}$ & $\begin{aligned} T^{2} & =0 \\
F R & =R F\end{aligned}$ & $\begin{array}{l}R F=F R \\
R^{3}=0 \quad F^{2}=0 \\
R T=T R^{2} \\
T F=F T\end{array}$ \\
\hline
\end{tabular}

Figure 81. Ada and Sophie's group presentations for water (left), ammonia (center), and ethane (right).

So when asked to create a system for determining groups, to Ada and Sophie this meant a creating a system that produced group presentations as seen in the following exchange:

Annie: So we want to do is come up with a system for say chemistry people or somebody else, who doesn't want to do all the group theory in the middle, they just want to go from; I have a molecule, through some kind of algorithm or flow-chart, to here's its group.

Ada: So their end group... they would desire...

Annie: So what was the most useful thing for you guys to know the whole group? What is the most sort of compact information that we can give them so that they know the whole group?

Ada: The rules!

Sophie: Yeah, the rules. 
Ada and Sophie successfully produced a system that helped produce the group presentation for the symmetry group of a given molecule, found in Figure 82 below.

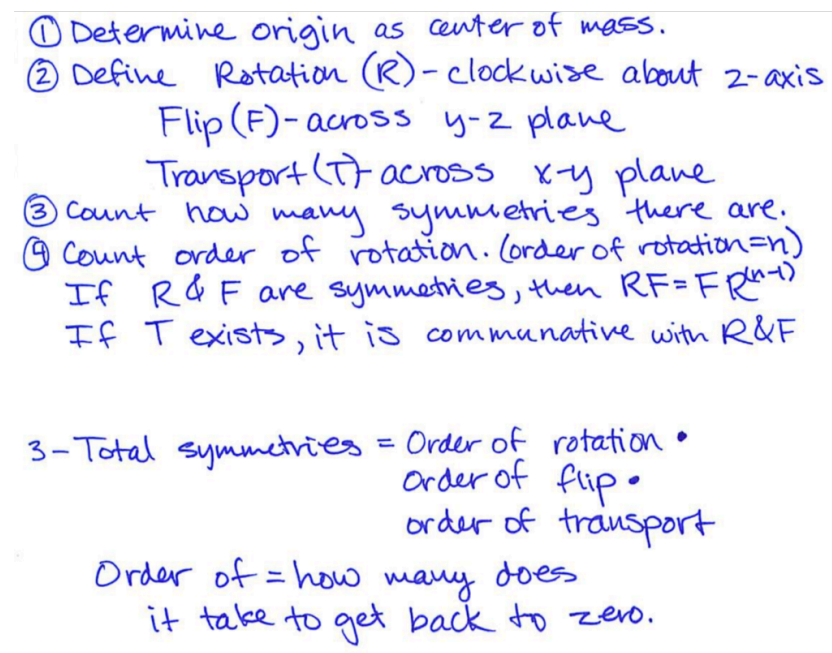

Figure 82. Ada and Sophie's initial model for identifying the symmetry group for a given molecule.

At this point it was becoming clear that Sophie and Ada needed a more concise representation for their symmetry groups in order to serve as the outputs of their classification system (model 2). I decided to ask Ada and Sophie to test their newly developed system on a new set of molecules seen in Figure 83.

\begin{tabular}{|c|c|c|c|c|}
\hline Tetra-aza copper II & $\begin{array}{l}\text { Hydrogen } \\
\text { peroxide }\end{array}$ & Boric acid & $\mathrm{BrF}_{5}$ & cyclobutene \\
\hline $\mathbb{Z}_{4}$ & $\mathbb{Z}_{2}$ & $\mathbb{Z}_{3} \times \mathbb{Z}_{2}$ & $\mathrm{D}_{8}$ & $\mathrm{D}_{8} \times \mathbb{Z}_{2}$ \\
\hline $\begin{array}{l}T A C \\
R^{4}=0 \quad 4 \text { symmetries } \\
\text { No } F_{s} \text { No } T s\end{array}$ & $\begin{array}{c}\text { Hydrogen Peroxide } \\
\qquad R^{2}=0\end{array}$ & $\begin{array}{l}\text { Boric Acid } \\
R^{3}=0 \quad T^{2}=0 \\
6 \text { symmetries } \\
T R=R T\end{array}$ & $\begin{array}{l}\text { Brain Slug } \\
4 \text { rotations } \\
R^{4}=0 \quad F^{2}=0 \\
8 \text { symmetries } \\
R F=P R^{3}\end{array}$ & $\begin{array}{l}\text { Cyclobutane } \\
R^{4}=0 \quad F^{2}=0 \quad T^{2}=0 \\
16 \text { symmetries } \\
R F=F R^{3} \\
T R=R T \\
T F=F T\end{array}$ \\
\hline
\end{tabular}

Figure 83. Additional molecules given to Ada and Sophie, and the group presentations they produced for each. 
This new set of molecules were specifically chosen to expose Ada and Sophie to chemically relevant group structures that they didn't yet have experience with. The five molecules in Figure 83 give students an opportunity to work with each of the most common group structures found in chemically important symmetry groups: cyclic, dihedral, cyclic $x \mathbb{Z}_{2}$, and dihedral $x \mathbb{Z}_{2}$. This activity also allowed Ada and Sophie an opportunity to gain more confidence in their system and again exposed them to additional group structures. This exposure to additional group structures was important step in the further evolution of Ada and Sophie's emergent model of their classification system. Working with these types of structures also encouraged the continual shift in Ada and Sophie's mathematical activity towards increasingly formal mathematics.

In order to support Ada and Sophie in developing group names to replace/represent their group presentations, we decided on a sorting activity. The goal was to see if Ada and Sophie would sort the different group presentations into categories defined by their group structure. The new sorting activity had 3 parts:

1. Make a score card for each of the molecules

2. Sort the score cards into collections/flavors/kinds of symmetry groups. Which seems more similar? Which hangout together?

3. Come up with a name for each of the collections.

Part 1: Make score cards for each molecule

To start the sorting task Ada and Sophie were asked to make "score cards" for each of the molecules they had encountered so far in other words a list of all the relevant information they found for the symmetry group, in other words their group presentations. 
This was eight molecules in total, five they had just completed in the previous task, and the three initial molecules. Creating the score cards involved going though previous work to find their group presentations. Doing so gave Ada and Sophie an opportunity to both standardize their notation and streamline their presentations. A clear example of this can be seen by comparing their group representation for water in Figure 81 above and their score card for water in Figure 84 below.

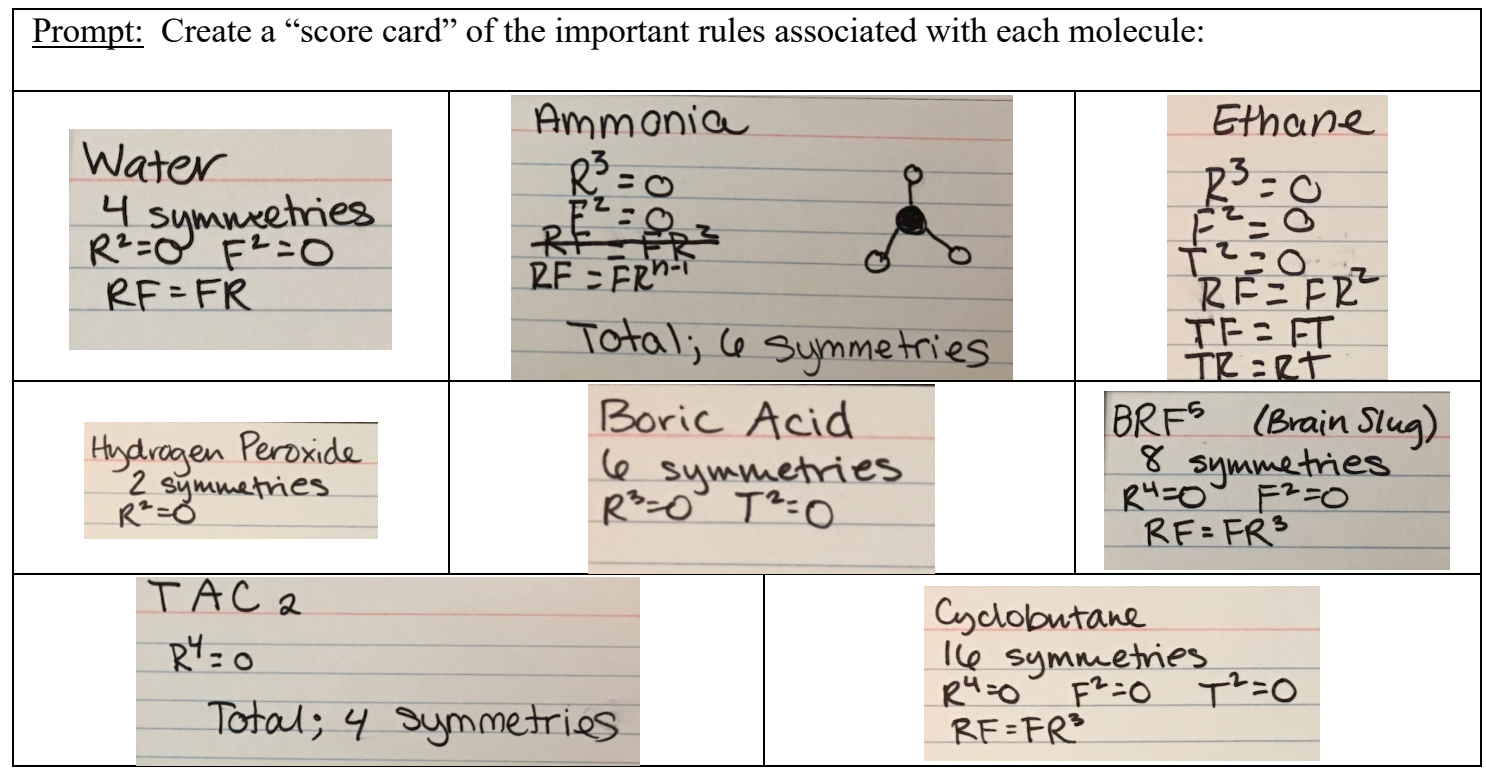

Figure 84. Ada and Sophie's "score cards" for each molecule containing its symmetry group presentation

Part 2: Sort the cards into types of symmetry groups.

When asked to sort the score cards into collections of similar groups types Ada and Sophies initially offered two different strategies. Ada starts by gathering all the pure rotational groups together, whereas Sophie gathers all the groups with order 3 rotations. Sophie speaks first suggesting that they put all the groups with the same order rotation together, but then quickly stops saying:

Sophie: Oh wait, what about the F's and the T's? 
Ada: That's kinda where I was thinking, if we group together.

Sophie: Well this is an F and a T, that's just an R and T, this is and F and a T...

I quickly remind them that with that strategy there will be 8 groups total because of the restriction on order of rotational groups due to chemical constraints which we had previously discussed. Then we had the following exchange:

Annie: If we go back to your instructions the fact that you wrote "if F" "if T exists" it makes me feel like it's important whether or not you know whether or not it has one of these kinds of symmetries or another.

Sophie: Yeah.

Ada: Yeah.

Annie: Right, once I know the order of rotation, I know what to do with it (indicating to their instructions for determining a group). But really if something exists or not, to me that holds a little more weight.

Sophie: Yeah, that does, that's kinds of important. Not all of them have T's.

Ada: I kinda inherently wanna be like, oh there are ones that have R's and F's , R's and T's, R's, T's, and F's, or just R's.

Sophie: Yeah, it seems, it's totally grouping them.

Ada: I think that would be like my gut instinct.

They proceed to group the score cards by existence of symmetries, seen in Figure 85 . 


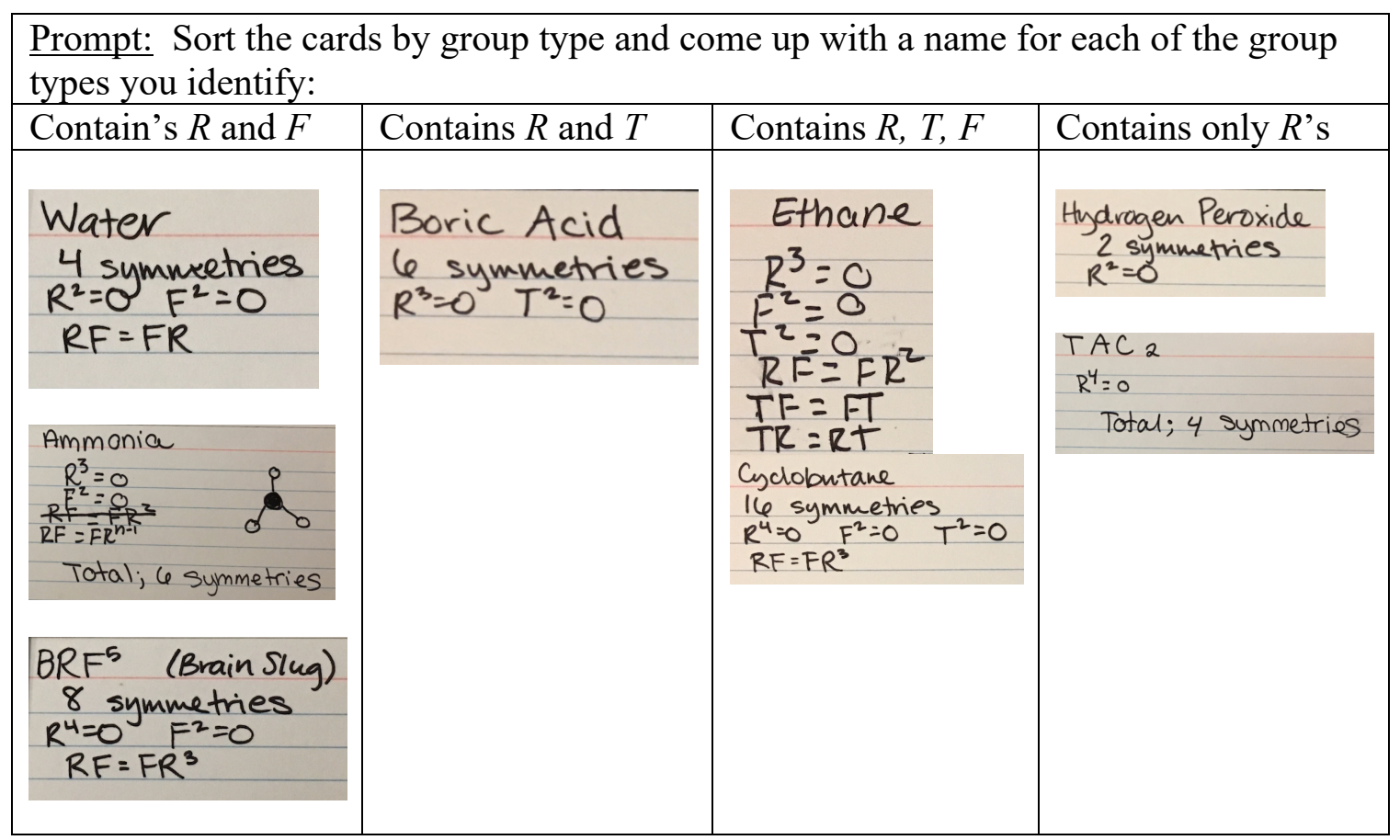

Figure 85. Ada and Sophie's sorting of groups into group types by existence of symmetries.

Once sorted into four collections I ask:

Annie: How does that feel?

Ada: Visually I think that they look different, like just oh gee what do these two have in common, (motions to Tetra-aza Copper II and hydrogen peroxide), versus oh gee what do these two have in common (motions to water and hydrogen peroxide), it's harder to picture, but it also, I feel like it means you're gaining more when you're dividing it into a group because you're grouping things together in a different way some way that you wouldn't just snap decision and look at.

Annie: It's not quite as superficial is it?

Ada: Yeah

Sophie: Yeah 
Ada: You probably gain more information with that (motions to new grouping).

Part 3: Create names for the four types of symmetry groups.

Once Ada and Sophie were comfortable with their grouping of the groups, they I asked them if they could name each of the groups, and they immediately named them after the collection of symmetries they contain thus creating the 4 symmetry group types in Figure 86 below.

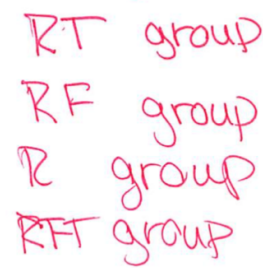

Figure 86. Ada and Sophie's group names

Armed with group names, Ada and Sophie are quickly able to redesign their system for identifying the symmetry group of a molecule (model 1) into more of a decision tree or flowchart where the newly created group names serve as outputs. Ada and Sophie each create their own system seen in Figure 87 below.

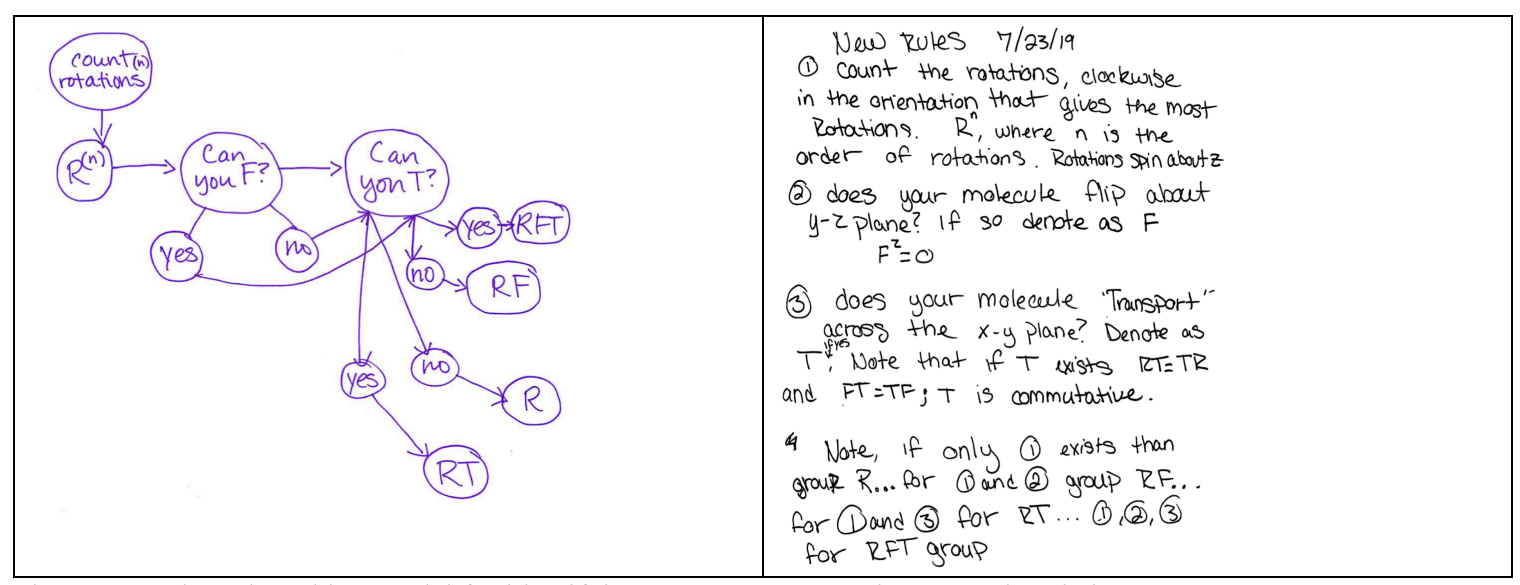

Figure 87. Ada and Sophie's model for identifying symmetry groups incorporating their new group names.

Summary of Case 2: Ada and Sophie 
As the researcher, in order to support Ada and Sophie in describing symmetry groups with concise group representations, I provided supplemental tasks and prompts to help scaffold their learning. Again, in order for the students in TE3 to be successful in completing Step 2 of the LIT I faced a design challenge of how best to support them. Whereas in TE2 the additional tasks created resulted in many lessons learns and ideas to move forward, I will argue that the results of TE3 were a success. The creation of the sorting task was a successful answer to the question; how do I support students in naming the outputs of this process, when considering options, is there any way to turn an apparent weakness of not having access to the conventional approach to groups into a strength? Is there a way I can leverage their informal mathematical activity? Ada and Sophie were extremely proficient at creating group presentations and so the sorting task was a natural way to build off that strength and help them find success in the LIT, and it worked very well.

\section{Conclusions}

In this paper I have presented the mathematical activity of two sets of students who were supported in their use of encapsulated group representations, aka group names, in a series of teaching experiments. The mathematical backgrounds of the two pairs of students were very different and this led to very different approaches to the same mathematical task. In the first case in TE2, the students wanted to use standard group names, but needed support in correctly arguing the symmetry groups they were observing were equivalent. The students did however, provide an interesting and powerful intuitive notion of direct product that were leveraged in a series of supplemental tasks. The tasks 
allowed the students to both gain more experience with direct products and also strengthen their understanding of isomorphisms.

This student approach differed a lot from the mathematical activity of the students in TE3. In the second case described above, the students needed additional scaffolding to help them further encapsulate their group representations into group names. Leveraging the group representation that the students were already comfortable with, I created a series of supplemental tasks that led the students to reinvent group names. In both instances I reacted to the students' initial approaches, accounted for their mathematical backgrounds and helped them successfully continue through the LIT.

There are important lessons to be learned from each of the two cases presented here. Arthur and Stu's activity suggest that the tasks associated with the LIT might also provide a fruitful context for developing students' informal thinking on direct and semi direct products. In the end their notion of a direct product with $\mathbb{Z}_{2}$ as a color swap and Stu's description of a semi-direct product as a twisting of the labels, were exciting glimpses at concepts that are arguably absent from current mathematics education literature. Their activity also suggests that establishing isomorphisms between groups outside of the context of a group theory course is non-trivial for students. Arthur and Stu were both strong mathematics students coming into the teaching experiment and being asked to transfer that formal knowledge into a new and unfamiliar setting was a challenge for them. While they had bits and pieces of what needed to happen in the abstract to make the arguments they wanted, ultimately their dependence on orientations kept them somewhat tangled up and tied down. Arthur and Stu offered an extremely valuable lesson for the LIT overall, that is that students must be thinking about symmetries as 
transformation in order to fully engage with the LIT and successfully classify symmetry groups. If students continue to conceptualize symmetries as the result of an operation instead of the operation itself, they will also continue to struggle with establishing correspondences beyond labeling.

As for Case 2, Ada and Sophie showed how students without previous group theory knowledge could reinvent something like a group name, the most popular group representation. Their method of using increasingly encapsulated group representations is also an exciting example of a chain of signification where each successive representation signifies the previous representation, see Chapter 6 for more detail. Chains of signification are important instantiations of the evolution of the students' mathematical activity during the reinvention process. They are also further evidence of the shift from the students use of a model of their situated mathematical activity to a model for more formal mathematical situations.

The most important lesson to learn is from both cases together. Together they offer examples of ways in which I responded to my students' mathematical activity. In both cases the students needed additional support in order to successfully use an appropriate group representation, and so I developed two very different sets of supplemental tasks based on the mathematical backgrounds of the students, both of which were used to help students find success to the same LIT. This experience of working with students who had different level of experience shed light on the challenges and opportunities involved in using a LIT to design instruction for different contexts. While designing supplemental tasks it was important to build on the mathematics that the students were offering. For example in Case 2 Ada and Sophie were very comfortable with making group 
presentations and so using group presentations as a starting point to reinvent group names seemed very natural to them. Whereas in Case 1 Arthur and Stu never offered group presentations and so the sorting task used in Case 2 would have been foreign to them.

Working with students who had varying exposure to group theory specifically was also extremely illuminating. The students who arguably struggled the least and appeared most comfortable participating in the reinvention process were Ada and Sophie, who had no previous experience with group theory. All of the students who had studied group theory and/or abstract algebra previously often struggled with translating their group theory knowledge to a chemistry context. The lack of abstraction seemed to be a bit of a hurdle for them and it was really interesting to see the difference in levels of comfort between those who were used to abstraction and those who had only seen group theory in context. I think this speaks to a larger question of how students are meant to "apply" what they learn in a traditional group theory course to situations beyond the course? The two cases presented here share successes, partial successes, and lesson learned from applying the LIT to different contexts where students engaged with the mathematics very differently. 
Chapter 8: Conclusions

For my dissertation study, I completed an instructional design project with the purpose of creating a local instructional theory which engaged students in deepening their understanding of group theory using a real-world context. The main research question guiding the study was:

\section{How can students be supported in reinventing an algorithm for the classification of chemically important symmetry groups?}

To answer this question I conducted three teaching experiments with pairs of mathematics students with varying mathematical backgrounds. The first teaching experiment was conducted with a pair of mathematics education graduate students who both had lots of previous experience with abstract algebra including experience in classifying finite groups. The findings from this experiment served as an existence proof of how students might engage in the reinvention process which informed the preliminary local instructional theory that was then tested and refined in the following two teaching experiments. The mathematical activity of the students in TE1 is described in detail in the first paper Chapter 5: Paper 1: Developing an Active Approach to Chemistry Based Group Theory. Paper 1 can be considered a practitioner paper as it was written for a chemistry education audience and recently published in an American Chemical Society book titled It's Just Math: Research on Students' Understanding of Chemistry and Mathematics.

The second paper of my dissertation Chapter 6: Paper 2: A Local Instructional Theory 
for the Guided Reinvention of a Classification Algorithm for Chemically Important Symmetry Groups, is the main deliverable of the instructional design study, informed by the students' mathematical activity across all three teaching experiments. The local instructional theory, LIT, gives a generalized theory of how students could reinvent a classification system for chemically important symmetry groups that can be used to determine the symmetry group of any given molecule. The LIT is described in terms of the students' mathematical activity and is organized into the six steps identified as key during the reinvention process. Each step of the LIT includes a sample task or prompt that can be used to evoke the particular mathematical activity necessary and then also gives examples of specific mathematical activity observed during the teaching experiments. These examples of students' mathematics help to provide the empirical rationale for the step in the LIT and this empirical rationale is accompanied by theoretical rationale for the necessity of the step and how it fits in the reinvention process.

The elaboration of the LIT in Paper 2 uses primarily data from the third teaching experiment in which the participants had no previous exposure to abstract algebra. The overall mathematical activity of the students in TE3 was markedly different from the students in TE2 who had recently completed a standard introductory group theory course. Minor differences in their approaches are shared in Paper 2 to reinforce the idea that the LIT is a generalized theory as opposed to an instructional sequence. One major difference in the approaches of the participants it was caused by the differences in the mathematical backgrounds of the participants in TE2 and TE3. More specifically, in Step 2 of the LIT when the participants needed to encapsulate their group representation into something like a group name to serve as an output for their flowchart, the students in the 
TE2 who had access to standard group names had a very different approach than those in TE3 who again, without previous exposure to advanced mathematics did not have access to conventional representations. Even though the students in TE2 had much more access to standard group names than the students in TE3, as in at least they knew of their existence, the students in TE2 didn't have quite robust enough of an understanding of conventional group names to confidently establish isomorphisms with the symmetry groups they were observing.

In both TE2 and TE3 the students needed different but additional support in order to successfully complete Step 2 of the LIT, use group names, and continue along the LIT. Chapter 7: Paper 3: Productively Applying and Adapting a LIT for Different Kinds of Mathematical Preparation, describes the strengths and limitations in the students' initial approaches, and then explicates how I as the researcher responded to their mathematical activity. In TE2 the students offered interesting informal notions around direct products. However, once I had a better understanding of their thinking of direct products it became clear that they needed support in establishing isomorphisms. Paper 3 describes in detail a series of six supplemental tasks that were created in order to help the students in TE2 in establishing group equivalences. Being able to argue such equivalence allowed the students to then take ownership over the use of standard group names. Accompanying the supplemental tasks is a reflection of how successful each task was and what could be learned for future implementation of the LIT.

In TE3 the students simply didn't have access to standard group names due to their lack of previous exposure to group theory in general. Instead of using group names, the students in TE3 were comfortable and proficient with describing groups using group 
presentations. Paper 3 describes in detail the supplemental tasks given to students in TE3 to support them in reinventing an encapsulated group presentation equivalent to a group name. Paper 3 also starts to shed light on the important process of adapting LITs to the particular approaches and mathematics available to students with different mathematical backgrounds which is currently absent in the mathematics education literature.

Overall this dissertation study makes three main contributions to mathematics education. First and foremost this study was an instructional design experiment, and as such it produced an instructional theory for engaging students in an application-based approach to group theory. This theory of how to engage students in the reinvention of a classification system for chemically important symmetry groups is useful because it allows students to investigate important ideas in abstract algebra in a real-world context. Furthermore, by leveraging students' informal approaches in the creation of more formal mathematics, the students are given an opportunity to reinvent both fundamental group theory concepts such as group names, and something much more advanced, a classification system for symmetry groups.

The second contribution from this study is insight on how to adapt and apply a LIT for students with varying mathematical backgrounds. A clear distinction is made in mathematics education literature between local instructional theories and instructional sequences, and it is often stressed that LITs should be more general than a specific instructional sequence. However, there currently does not exist explicit evidence in the literature of how a user might adapt an existing LIT for varying populations. Paper 3 shares successes, partial successes, and lesson learned from applying the LIT to different contexts where students engaged with the mathematics very differently. 
The third and final contribution from this study is the beginnings of a conversation between two disciplines on how we might support even more student understanding. While the focus of this study was always to learn how to support mathematics students in their understanding of abstract algebra, it is reasonable to wonder how this LIT might support chemistry students as well. As mentioned, group theory is quite prevalent in undergraduate inorganic chemistry often introduced formally and from the perspective of an expert. My experience in TE3 working with students without previous exposure to abstract algebra suggests that the LIT could be implemented with chemistry students who also probably haven't studied group theory formally.

My dissertation naturally leads to two main branches of future research stemming from these results, the first is locally related to the LIT and the second considers the LIT in a more global sense. While my dissertation has produced evidence to support a LIT, I look forward to continuing its refinement and furthering its development. I would like to start by conducting a follow-up study that extends the investigation to include very high order symmetry groups, e,g, the Platonic solids. Future development of the LIT would also include learning how to support students in generating and structuring arguments for the completeness and generality of their classification algorithm. The results of the dissertation have also shown that the LIT can successfully engage students with a variety of mathematical backgrounds, therefore it has the potential to be implemented in a variety of contexts. In its current state this LIT could easily be incorporated into any abstract algebra course as an application module. However, due to the laundry list of prerequisites traditionally required for students to register for an abstract algebra course and given that these materials have been designed to support students without said pre- 
requisites, I would like to work to make this LIT available to a much broader audience. I see this LIT fitting into an inquiry-oriented, application-based group theory course for any STEM major regardless of previous advanced mathematical experience.

A classification algorithm identifying the symmetry group of a molecule is the first of two important mathematical artifacts used in undergraduate inorganic chemistry. Once the symmetry group of a molecule is determined, chemists refer to a particular group representation called a 'character table'. Character tables describe further characteristics a molecule may exhibit including for example modes of vibration. Character tables are constructed using matrix representations of symmetry groups and have the potential to engage students with advanced linear algebra in an authentic application. I am currently conducting a study looking at students' ability to move between various representations of linear systems. My preliminary findings show traditional linear algebra instruction may not be supporting students in understanding the various representations of matrices, and an instructional approach that guides students in reinventing character tables might be one way to address these struggles head on. I plan to develop a LIT for the guided reinvention of character tables and together these LITs will feed into an applied algebra course leveraging the context of chemistry. 
References

Almeida, D. F. (1999). Visual aspects of understanding group theory. International Journal of Mathematical Education in Science and Technology, 30(2), 159-166.

Asiala, M., Dubinsky, E., Mathews, D. M., Morics, S., \& Oktaç, A. (1997). Development of students' understanding of cosets, normality, and quotient groups. The Journal of Mathematical Behavior, 16(3), 241-309.

Asiala, M., Kleiman, J., Brown, A., \& Mathews, D. (1998). The development of students' understanding of permutations and symmetries. International Journal of Computers for Mathematical Learning, 3(1), 13-43.

Benatan, E., Dene, J., Eppley, H. J., Geselbracht, M. J., Jamieson, E. R., Johnson, A. R., ... Williams, B. S. (2009). JCE VIPEr: an inorganic teaching and learning community. Journal of Chemical Education, 86(6), 766-767.

Bergman, A., \& French, T. (2019). Developing an Active Approach to Chemistry-Based Group Theory. In It's Just Math: Research on Students' Understanding of Chemistry and Mathematics (pp. 213-237). American Chemical Society.

Blair, R., Kirkman, E. E., and Maxwell, J. W. (2013), Statistical Abstract of Undergrtaduate Programs in the Mathematical Sciences in the United States: Fall 2010 CBMS Survey, Washington, D.C.: American Mathematical Society.

Brown, A., DeVries, D. J., Dubinsky, E., \& Thomas, K. (1997). Learning binary operations, groups, and subgroups. The Journal of Mathematical Behavior, 16(3), 187-239.

Burn, B. (1996). What are the fundamental concepts of group theory? Educational Studies in Mathematics, 31(4), 371-377.

Carroll, J. B. (1993). Human Cognitive Abilities: A Survey of Factor-Analytic Studies (pp. 304-363). Cambridge, England: Cambridge University Press.

Cobb, P., \& Bauersfeld, H. (1995). The emergence of mathematical meaning: Interaction in classroom cultures (Studies in mathematical thinking and learning). Hillsdale, N.J.: L. Erlbaum Associates.

Cobb, P., Confrey, J., DiSessa, A., Lehrer, R., \& Schauble, L. (2003). Design experiments in educational research. Educational Researcher, 32(1), 9-13.

Cook, J.P. (2012a). A Guided Reinvention of Ring, Integral Domain, and Field. (Doctoral dissertation). Available from ProQuest Dissertations and Theses database. (Order No. 3517320)

Cooper, M. M., Corley, L. M., \& Underwood, S. M. (2013). An investigation of college chemistry students' understanding of structure-property relationships. Journal of Research in Science Teaching, 50(6), 699-721.

Cooper, M. M., Underwood, S. M., Hilley, C. Z., \& Klymkowsky, M. W. (2012). Development and Assessment of a Molecular Structure and Properties Learning Progression. Journal of Chemical Education, 89(11), 1351-1357.

Cotton, F. A., \& Wilkinson, G. (1988). Advanced inorganic chemistry (Vol. 545). Wiley, New York.

Design-Based Research: An Emerging Paradigm for Educational Inquiry. (2003). Educational Researcher, 32(1), 5-8. 
Dubinsky, E., Dautermann, J., Leron, U., \& Zazkis, R. (1994). On learning fundamental concepts of group theory. Educational Studies in Mathematics, 27(3), 267-305.

Dubinsky, E., Henderson, D. W., \& Nosrati, M. (2017). Equivalence of Symmetries and Permutations in R $\mathrm{n}$. The Mathematical Intelligencer, 39(1), 53-59.

Flint, E. B. (2011). Teaching point-group symmetry with three-dimensional models. Journal of Chemical Education, 88(7), 907-909.

Freudenthal, H. (1971). Geometry between the devil and the deep sea. Educational Studies in Mathematics, 3(3-4), 413-435.

Freudenthal, H. (1973). What groups mean in mathematics and what they should mean in mathematical education. In A. G. Howson (Eds.), Developments in Mathematical Education, Proceedings of ICME-2 (pp. 101-114). Cambridge University Press, Cambridge, U.K.

Freudenthal, H. (1986). Didactical Phenomenology of Mathematical Structures. Springer. Freudenthal, H. (1991). Revisiting mathematics education: China Lectures. Hingham, MA: Kluwer Academic Publishers.

Galton, S. F. (1880). Mental Imagery. Mind, 5(19), 301-318.

Grafton, A. K. (2011). Using role-playing game dice to teach the concepts of symmetry. Journal of Chemical Education, 88(9), 1281-1282.

Gravemeijer, K. (1998). Developmental research as a research method. In A. Sierpinska \& J. Kilpatrick (Eds.), Mathematics Education as a Research Domain: A Search for Identity (pp. 277-295). Dordrecht, The Netherlands: Kluwer.

Gravemeijer, K. (2002) Preamble: From Models to Modeling. In Gravemeijer K., Lehrer R., Van Oers B., Verschaffel L., (Eds.), Symbolizing, Modeling and Tool Use in Mathematics Education (pp. 7-22). Springer, Dordrecht.

Gravemeijer, K. (2004). Local instruction theories as means of support for teachers in reform mathematics education. Mathematical Thinking and Learning, 6(2), 105128.

Gravemeijer, K., \& Doorman, M. (1999). Context problems in Realistic Mathematics Education: A calculus course as an example. Educational Studies in Mathematics, 39(1), 111-29.

Gravemeijer, K., \& Stephan, M. (2002). Emergent models as an instructional design heuristic. In Symbolizing, modeling and tool use in mathematics education (pp. 145-169). Springer.

Gravemeijer, K., \& Terwel, J. (2000). Hans Freudenthal: A mathematician on didactics and curriculum theory. Journal of Curriculum Studies, 32(6), 777-796.

Gravemeijer, K., \& van Eerde, D. (2009). Design research as a means for building a knowledge base for teachers and teaching in mathematics education. The Elementary School Journal, 109(5), 510-524.

Grove, N. P., Guerin, N. P., Collins, D. J., López, J. J., Bretz, S. L., \& Zhou, H.-C. (2009). Designing, teaching, and evaluating a unit on symmetry and crystallography in the high school classroom. Journal of Chemical Education, 86(8), 946.

Habraken, C. L. (2004). Integrating into chemistry teaching today's student's visuospatial talents and skills, and the teaching of today's chemistry's graphical language. Journal of Science Education and Technology, 13(1), 89-94. 
Harle, M., \& Towns, M. (2010). A review of spatial ability literature, its connection to chemistry, and implications for instruction. Journal of Chemical Education, 88(3), 351-360.

Hazzan, O. (1999). Reducing abstraction level when learning abstract algebra concepts. Educational Studies in Mathematics, 40(1), 71-90.

Hazzan, O., \& Leron, U. (1996). Students' use and misuse of mathematical theorems: The case of Lagrange's theorem. For the Learning of Mathematics, 16(1), 23-26.

Ioannou, M., \& Nardi, E. (2009). Engagement, abstraction and visualisation: Cognitive and emotional aspects of Year 2 mathematics undergraduates' learning experience in Abstract Algebra. Proceedings of the British Society for Research into Learning Mathematics, 29(2), 35-40.

Johnson, Caughman, Fredericks, \& Gibson. (2013). Implementing inquiry-oriented curriculum: From the mathematicians' perspective. Journal of Mathematical Behavior, 32(4), 743-760.

Larive, C. K., \& Polik, W. F. (2008). New ACS guidelines approved by CPT. ACS Publications.

Larsen, S. (2004). Supporting the guided reinvention of the concepts of group and isomorphism: A developmental research project (Doctoral dissertation, Arizona State University, 2004). Dissertation Abstracts International, B, 65(02), 781.

Larsen, S. (2009). Reinventing the concepts of group and isomorphism: The case of Jessica and Sandra. The Journal of Mathematical Behavior, 28(2), 119-137.

Larsen, S. (2010a). TAAFU: Teaching Abstract Algebra for Understanding. Retrieved from http://www.web.pdx.edu/ slarsen/TAAFU/unit1/lesson1/lesson1.php

Larsen, S. (2010b). Struggling to disentangle the associative and commutative properties. For the Learning of Mathematics, 30(1), 37-42.

Larsen, S. (2013). A local instructional theory for the guided reinvention of the group and isomorphism concepts. The Journal of Mathematical Behavior, 32(4), 712-725.

Larsen, S. (2018). Didactical phenomenology: the engine that drives Realistic Mathematics Education. For the Learning of Mathematics, 38(3), 25-29.

Larsen, S., \& Bartlo, J. (2009). The role of tasks in promoting discourse supporting mathematical learning. In L. Knott (Ed.), The role of mathematics discourse in producing leaders of discourse (pp. 77-98). Charlotte, NC: Information Age Publishing

Larsen, S., Johnson, E., \& Bartlo, J. (2013). Designing and scaling up an innovation in abstract algebra. Journal of Mathematical Behavior, 32(4), 693-711.

Larsen, S., \& Lockwood, E. (2013). A local instructional theory for the guided reinvention of the quotient group concept. The Journal of Mathematical Behavior, 32(4), 726-742.

Leron, U., \& Dubinsky, E. (1995). An abstract algebra story. American Mathematical Monthly, 227-242.

Leron, U., Hazzan, O., \& Zazkis, R. (1995). Learning group isomorphism: A crossroads of many concepts. Educational Studies in Mathematics, 29(2), 153-174

Lohman, D. F. (1979). Spatial Ability: A Review and Reanalysis of the Correlational Literature. Technical Report No. 8, Aptitude Research Project; School of Education, Stanford University: Palo Alto, CA. 
Luxford, C. J., Crowder, M. W., \& Bretz, S. L. (2011). A Symmetry POGIL Activity for inorganic chemistry. Journal of Chemical Education, 89(2), 211-214.

Marek, Keith A., Raker, Jeffery R., Holme, Thomas A., \& Murphy, Kristen L. (2018). The ACS Exams Institute Undergraduate Chemistry Anchoring Concepts Content Map III: Inorganic Chemistry. Journal of Chemical Education, 95(2), 233-237.

McKay, S. E., \& Boone, S. R. (2001). An Early Emphasis on Symmetry and a ThreeDimensional Perspective in the Chemistry Curriculum. Journal of Chemical Education, 78(11), 1487.

Melhuish, K. M. (2015). The Design and Validation of a Group Theory Concept Inventory (PhD Thesis). Portland State University.

Melhuish, K., Bergman, A., \& Czocher, J. (2018). Melhuish, K., Bergman, A. M., Czocher, J. (February 2018). Revisiting reducing abstraction in abstract algebra.

Proceedings of the $21^{\text {st }}$ Meeting of the MAA Special Interest Group on Research in Undergraduate Mathematics Education, San Diego, California.

Miessler, G., Fischer, Paul J, \& Tarr, Donald A. (2014). Inorganic chemistry. (Fifth edition / Gary L. Miessler, St. Olaf College, Paul J. Fischer, Macalester College, Donald A. Tarr, St. Olaf College. ed.). Boston: Pearson.

Moog, R. S., \& Spencer, J. N. (2008). POGIL: An overview. Process Oriented Guided Inquiry Learning (POGIL), 994, 1-13.

Nardi, 2000

Portnoy, N., Grundmeier, T. A., \& Graham, K. J. (2006). Students' Understanding of Mathematical Objects in the Context of Transformational Geometry: Implications for Constructing and Understanding Proofs. Journal of Mathematical Behavior, 25(3), 196-207.

Raker, J. R., Reisner, B. A., Smith, S. R., Stewart, J. L., Crane, J. L., Pesterfield, L., \& Sobel, S. G. (2015a). Foundation Coursework in Undergraduate Inorganic Chemistry: Results from a National Survey of Inorganic Chemistry Faculty. Journal of Chemical Education, 92(6), 973-979.

Raker, J. R., Reisner, B. A., Smith, S. R., Stewart, J. L., Crane, J. L., Pesterfield, L., \& Sobel, S. G. (2015b). In-Depth Coursework in Undergraduate Inorganic Chemistry: Results from a National Survey of Inorganic Chemistry Faculty. Journal of Chemical Education, 92(6), 980-985.

Rasmussen, C. (Ed.). (2007). An inquiry oriented approach to differential equations. Journal of Mathematical Behavior, 26(3), http://dx.doi.org/10.1016/j.jmathb.2007.10.001 [Special issue].

Rasmussen, C., \& Marrongelle, K. (2006). Pedagogical Content Tools: Integrating Student Reasoning and Mathematics in Instruction. Journal for Research in Mathematics Education, 37(5), 388-420.

Rasmussen, C., Keene, K. A., Dunmyre, J., \& Fortune, N. (2018). Inquiry oriented differential equations: Course materials. Retrieved from https://iode.wordpress.ncsu.edu/

Reisner, B. A., Eppley, H. J., Geselbracht, M. J., Jamieson, E. R., Johnson, A. R., Smith, S. R., Stewart, J. L., Watson, L.A., \& Williams, S. B. (2010). Building an online teaching community: an evolving tale of Communication, collaboration, and 
Chemistry. In Enhancing Learning with Online Resources, Social Networking, and Digital Libraries (pp. 309-330). Washington, D. C.: American Chemical Society. Scalfani, V. F., \& Vaid, T. P. (2014). 3D printed molecules and extended solid models for teaching symmetry and point groups. Journal of Chemical Education, 91(8), 1174-1180.

Sein, L. (2010). Dynamic Paper Constructions for Easier Visualization of Molecular Symmetry. Journal of Chemical Education, 87(8), 827-828.

Shubbar, K. E. (1990). Learning the visualisation of rotations in diagrams of three dimensional structures. Research in Science \& Technological Education, 8(2), 145154.

Southam, D. C., \& Lewis, J. E. (2013). Supporting alternative strategies for learning chemical applications of group theory. Journal of Chemical Education, 90(11), 1425-1432.

Steffe, L. P. (1991). The constructivist teaching experiment: Illustrations and implications. In E. von Glasserfeld (Ed.), Radical constructivism in mathematics education (pp. 177-194). Boston, MA: Kluwer Academic Press.

Steffe, L. P., \& Thompson, P. W. (2000). Teaching experiment methodology: Underlying principles and essential elements. Handbook of Research Design in Mathematics and Science Education, 267-306.

Stieff, M. (2007). Mental rotation and diagrammic reasoning in science. Learning and Instruction, 17(2), 219-234.

Strand, S. R. II. (2016). The intermediate value theorem as a starting point for inquiryoriented advanced calculus (Order No. 10129071). Available from Dissertations \& Theses@ Portland State University; ProQuest Dissertations \& Theses Global. (1814774329).

Swinyard, C. (2011). Reinventing the formal definition of limit: The case of Amy and Mike. The Journal of Mathematical Behavior, 30(2), 93-114.

Tall, D., Thomas, M., Davis, G., Gray, E., \& Simpson, A. (2000). What Is the Object of the Encapsulation of a Process? Journal of Mathematical Behavior, 18(2), 223-241.

Thompson, A.G. (1984) The Relationship of Teachers' Conception of Mathematics Teaching to Instructional Practice. Educational Studies in Mathematics, 15 (2), 105127.

Tuckey, H., Selvaratnam, M., \& Bradley, J. (1991). Identification and rectification of student difficulties concerning three-dimensional structures, rotation, and reflection. Journal of Chemical Education, 68(6), 460.

Van den Heuvel-Panhuizen, M., \& Drijvers, P. (2014). Realistic mathematics education. In Encyclopedia of mathematics education (pp. 521-525). Springer, Dordrecht.

Wawro, M., Zandieh, M., Rasmussen, C., \& Andrews-Larson, C. (2013a). Inquiry oriented linear algebra: Course materials. Retrieved from http://iola.math.vt.edu/

Wawro, M., Rasmussen, C., Zandieh, M., \& Larson, C. (2013b). Design research within undergraduate mathematics education: An example from introductory linear algebra. In T. Plomp \& N. Nieveen (Eds.), Educational design research-Part B: Illustrative cases (pp. 905-925). Enschede: SLO. 
Weber, K., \& Larsen, S. (2008). Teaching and learning group theory. Making the Connection: Research and Teaching in Undergraduate Mathematics Education, 73, 139.

Wilensky, U. (1991). Abstract meditations on the concrete and concrete implications for mathematics education. In I. Harel and S. Papert (Eds.), Constructionism (pp. 193203). Norwood, NJ: Ablex Publishing Corporation.

Wu, H. K., Krajcik, J. S., \& Soloway, E. (2001). Promoting understanding of chemical representations: Students' use of a visualization tool in the classroom. Journal of Research in Science Teaching: The Official Journal of the National Association for Research in Science Teaching, 38(7), 821-842.

Zandieh, M., \& Rasmussen, C. (2010). Defining as a mathematical activity: A framework for characterizing progress from informal to more formal ways of reasoning. Journal of Mathematical Behavior, 29, 57-75.

Zazkis, R., Dubinsky, E., \& Dautermann, J. (1996). Coordinating Visual and Analytic Strategies: A Study of Students' Understanding of the Group D4. Journal for Research in Mathematics Education, 27(4), 435-457.

Zeldin, M. (1966). An introduction to molecular symmetry and symmetry point groups. Journal of Chemical Education, 43(1), 17. 
Appendix A: Supplement to Chapter 6

Sample Task and Prompt for Step 5b.

Prompt: Describe the symmetry group of each of the following:

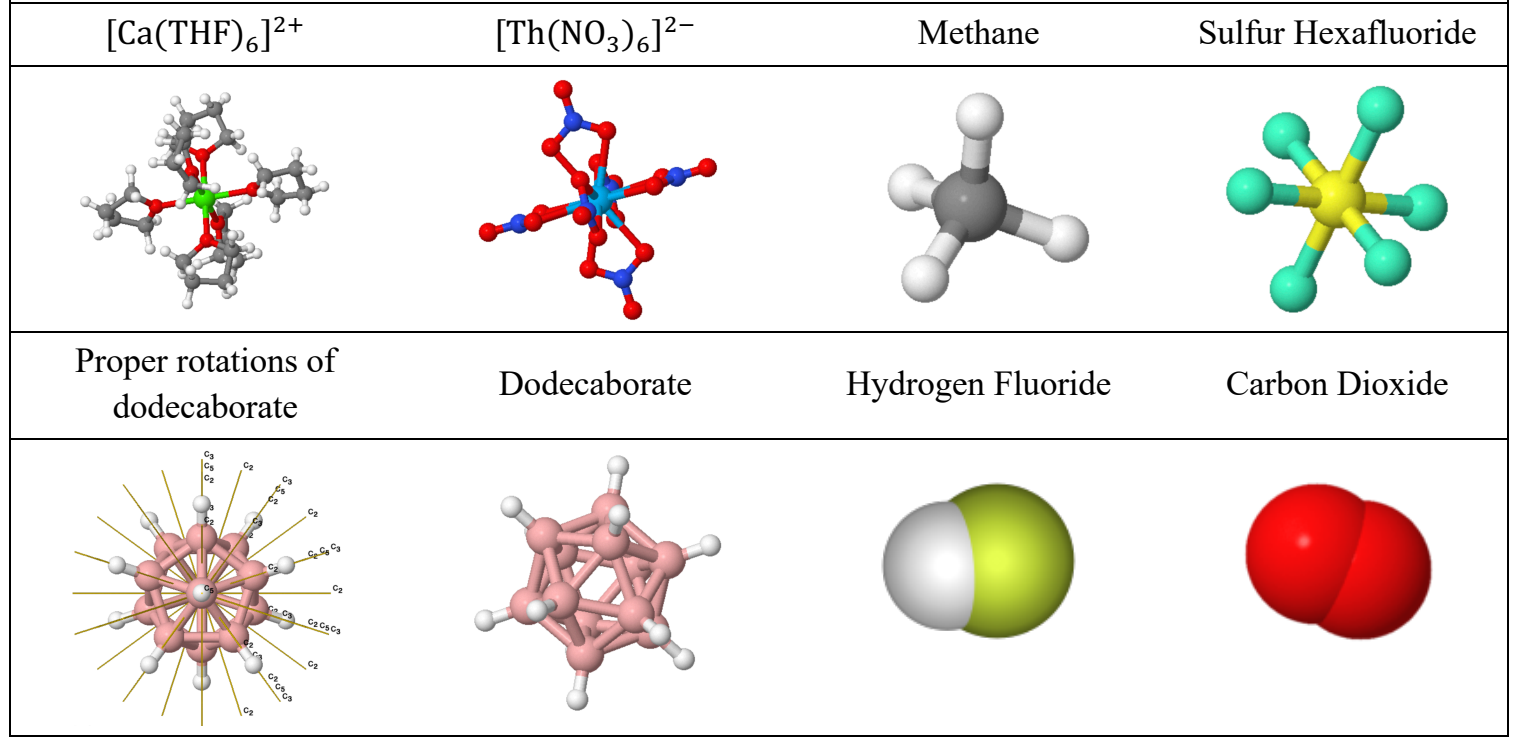

Below is a description of each of the molecules used in the task above and the most common mathematical group name for each.

The Cubic Groups; Tetrahedral and Octahedral

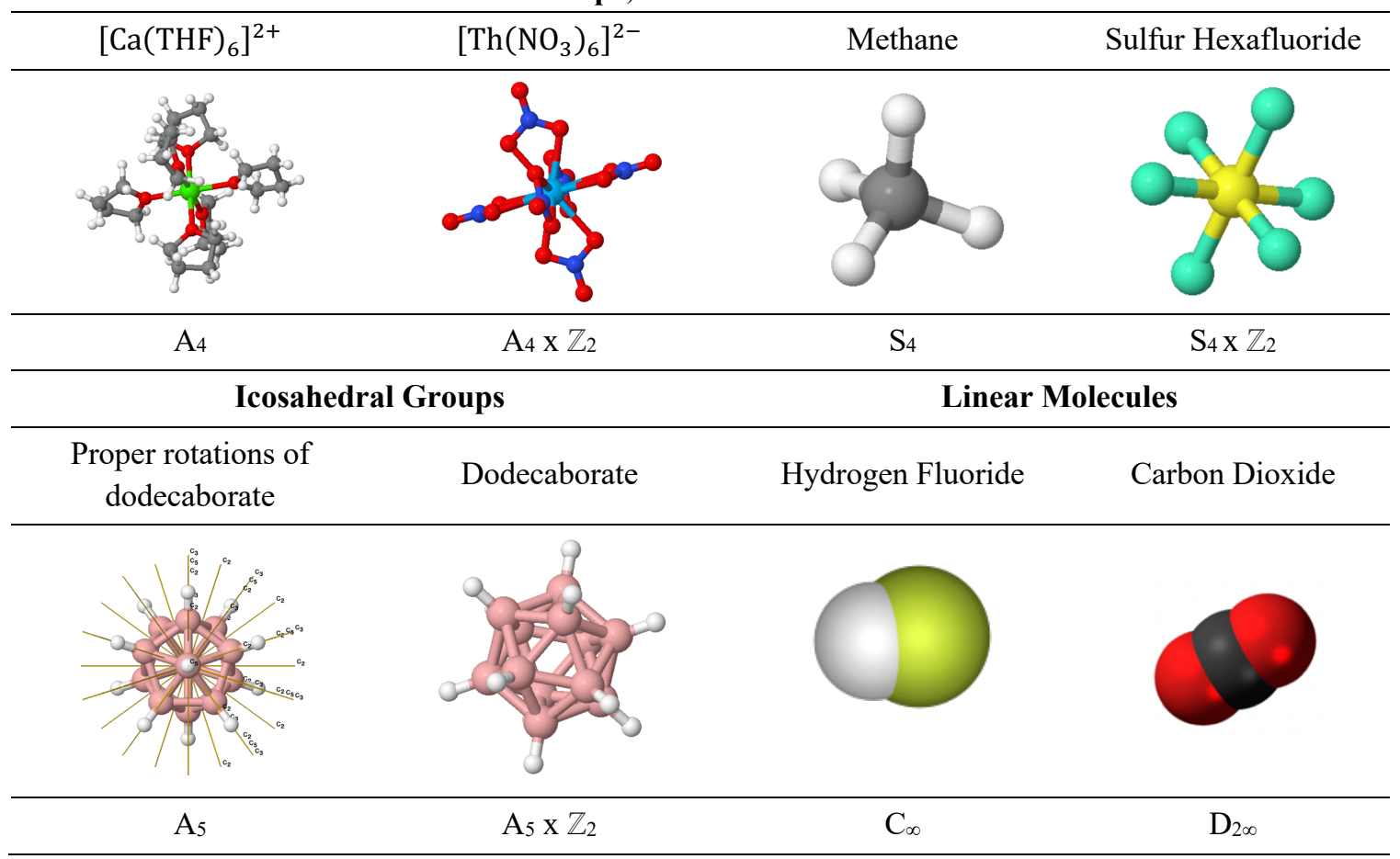




\section{Appendix B: Supplement to Chapter 7}

\begin{tabular}{|c|c|c|c|}
\hline & TE1 & TE2 & TE3 \\
\hline 1 & $60 \mathrm{~min}$ & $44 \mathrm{~min}$ & $78 \mathrm{~min}$ \\
\hline & $\begin{array}{l}\text { Design and describe an efficient } \\
\text { system for identifying the symmetry } \\
\text { group of any given molecule. } \\
\text { Begin by finding the symmetry group } \\
\text { of each of the given molecules; water, } \\
\text { ammonia, ethane, and methane. }\end{array}$ & $\begin{array}{l}\text { Rank, water, ammonia, ethane } \\
\text { (eclipsed), and TAC from most to least } \\
\text { symmetric. }\end{array}$ & $\begin{array}{l}\text { Start with the OG measuring } \\
\text { symmetry task } \\
-\quad \text { Define symmetry and } \\
\text { symmetry equivalence }\end{array}$ \\
\hline 2 & $75 \mathrm{~min}$ & $57 \mathrm{~min}$ & $93 \mathrm{~min}$ \\
\hline & $\begin{array}{l}\text { Generalize your method for finding } \\
\text { symmetry groups into a flowchart with } \\
\text { an instruction manual for someone } \\
\text { else. }\end{array}$ & OG measuring symmetry task & IOAA \\
\hline 3 & $80 \mathrm{~min}$ & $45 \mathrm{~min}$ & $105 \mathrm{~min}$ \\
\hline & $\begin{array}{l}\text { Re-write the flowchart so that it is } \\
\text { 1) easier to read } \\
\text { 2) correct } \\
\text { At this point there are terminal point } \\
\text { of the flowchart that do not match the } \\
\text { path used to get to them. } \\
\text { How do you know you have the } \\
\text { "right" rotation? } \\
\text { - In order for the students to } \\
\text { correspond the rotation in } \\
\text { their flowchart with the } \\
\text { highest order axis, we did } \\
\text { have to provide some } \\
\text { additional examples. } \\
\text { When pushing on } \\
\text { correctness, I pursued } \\
\text { multiple branches, } \\
\text { sometimes providing new } \\
\text { molecules with symmetry } \\
\text { groups } \mathbb{Z}_{4} \times \mathbb{Z}_{2} \text { and } \mathbb{Z}_{4} \text {. }\end{array}$ & $\begin{array}{l}\text { Define symmetry and symmetry } \\
\text { equivalence. }\end{array}$ & IOAA \\
\hline 4 & $75 \mathrm{~min}$ & $\mathrm{~N} / \mathrm{A}$ & $35 \mathrm{~min}$ \\
\hline & $\begin{array}{l}\text { While your flowchart is beautiful, do } \\
\text { you think that it captures everything? } \\
\text { Do you think it captures all the } \\
\text { symmetries of any given molecule? } \\
\quad \text { - They suspect it might not } \\
\text { work for staggered ethane } \\
\text { and start to identify the } \\
\text { symmetries while noticing } \\
\text { the inversion.) } \\
\text { I ask them to use the flowchart on } \\
\text { their 3-d ampersand. } \\
\text { I ask if they think they can identify the } \\
\text { symmetry group of any given } \\
\text { molecule using rotations, planes, } \\
\text { slices, and points? } \\
\text { I ask are there any other kind of } \\
\text { symmetry in 3-dimensional space? } \\
\text { How would you know? }\end{array}$ & $\begin{array}{l}\text { Find the symmetry group of for water, } \\
\text { ammonia, ethane, and TAC. }\end{array}$ & $\begin{array}{l}\text { IOAA } \\
\text { I give them the hooked sun and a non- } \\
\text { square rectangle. I ask them to make } \\
\text { symbols and tables for each of them. } \\
\text { I ask them to make a list of rules that } \\
\text { hold for all of their groups. }\end{array}$ \\
\hline 5 & & $94 \mathrm{~min}$ & $104 \mathrm{~min}$ \\
\hline
\end{tabular}




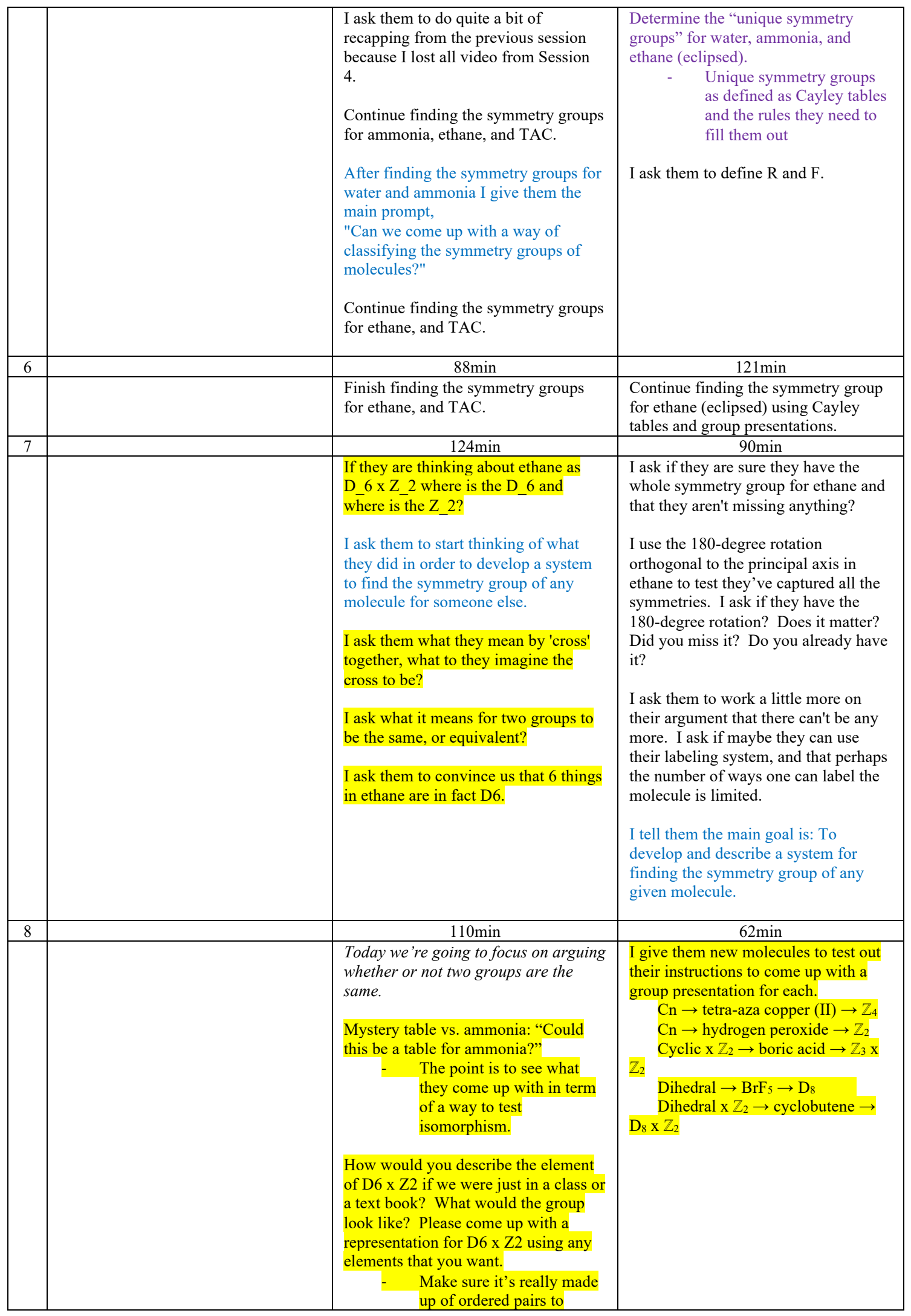




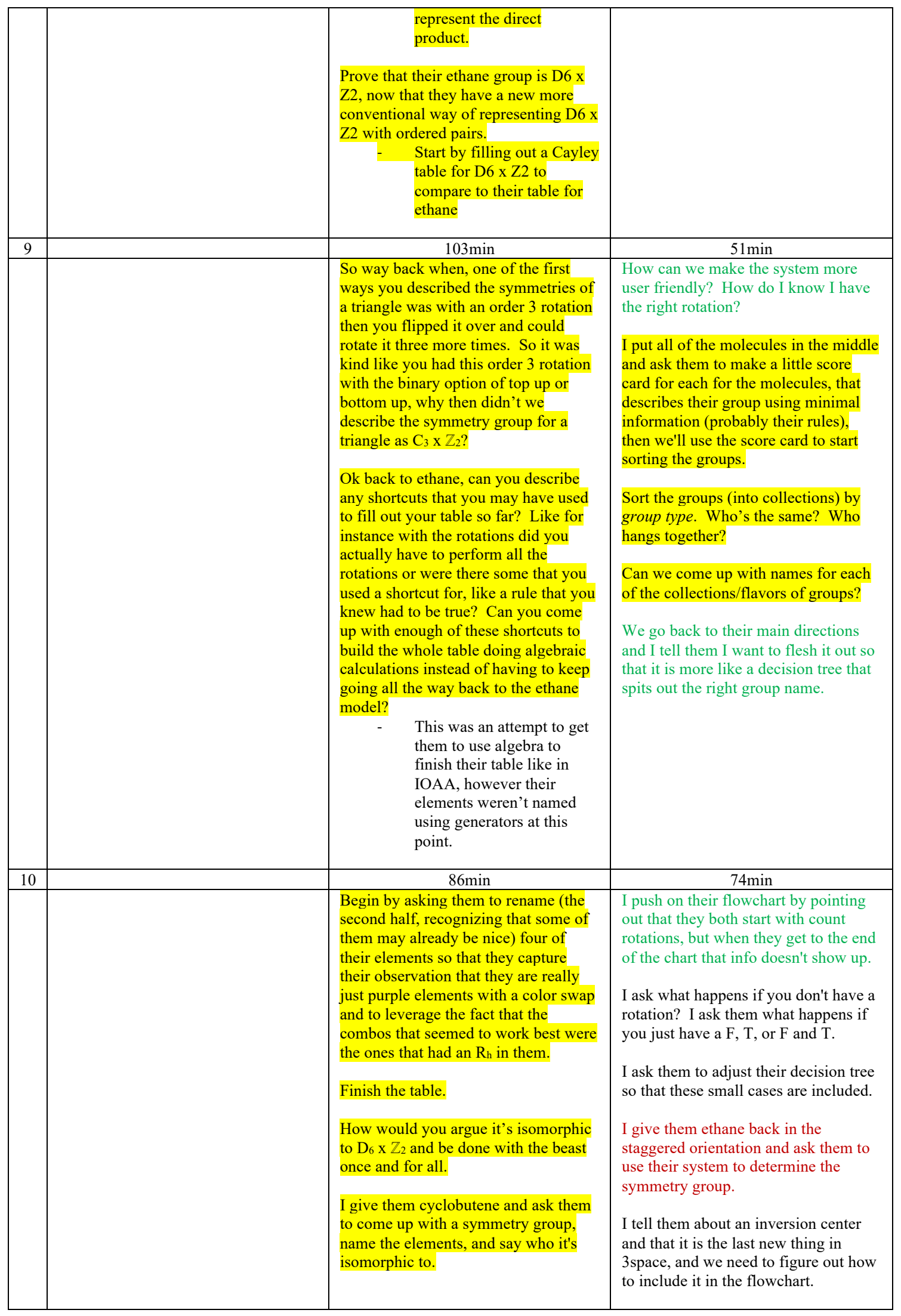




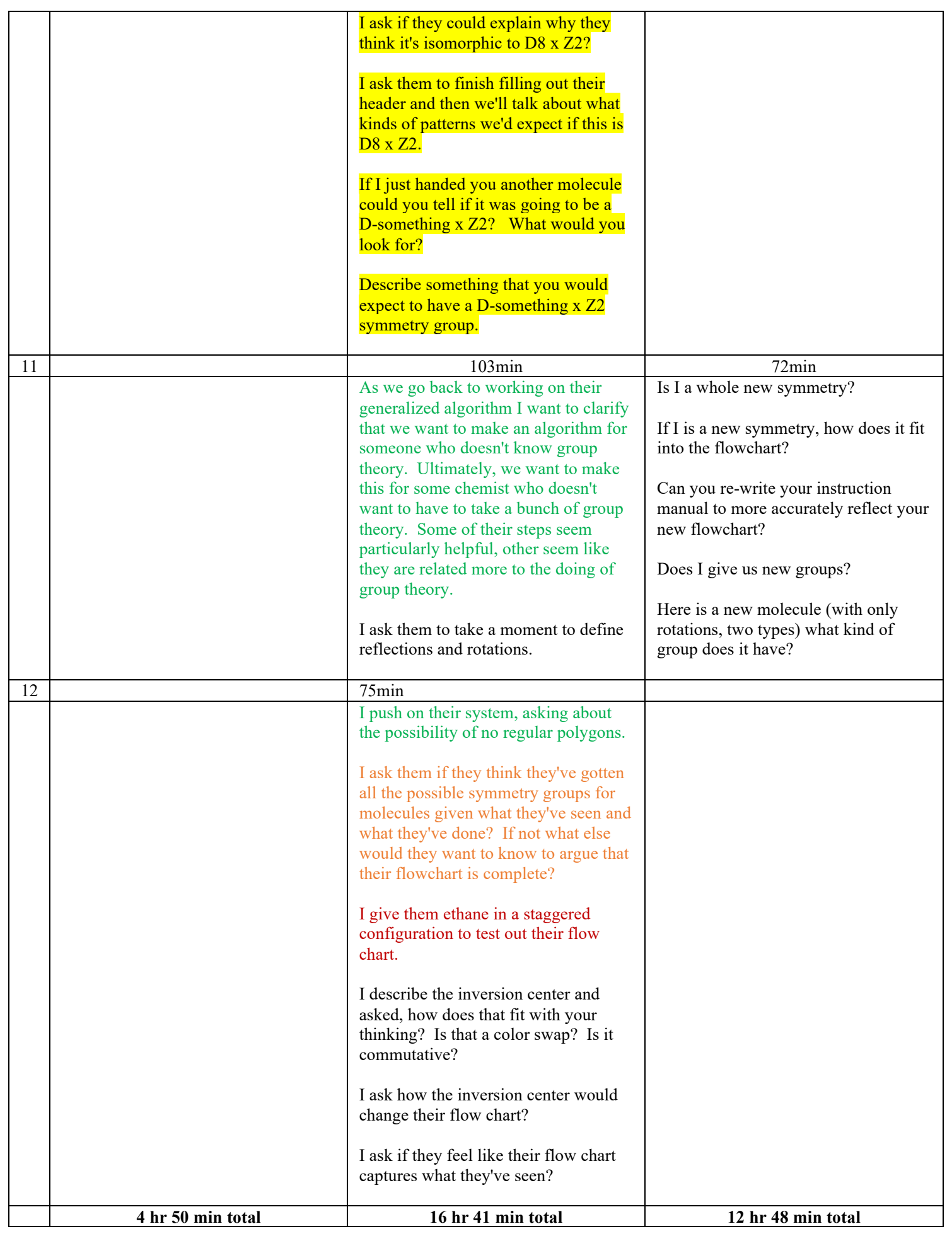




\section{Appendix C: Supplement to Chapter 7}

Let $G$ be a group such that $G=H K$, where $H \triangleleft G$ and $K \triangleleft G$ such that $H \cap K=\{e\}$, then $G \cong H \times K$.

pf: I will start by showing that every element of the group $G$ can be written uniquely as some $h k$, that is $\forall g \in G, g=h k \ni ! h \in H$ and $! k \in K$. Then I will prove that there exists an isomorphism from $G$ to $H \times K$, including a lemma showing that $h k=k h \forall h, k \in G$.

Claim: $\forall g \in G, g$ can be written uniquely as some $h k$, that is $\forall g \in G$, $g=h k \ni ! h \in H$ and $! k \in K$.

Let $g \in G$ we are given that $g=h k$ where $h \in H$ and $k \in K$.

Suppose $g=h_{l} k_{l}$ where $h_{l} \in H$ and $k_{l} \in K$ as well.

$$
\Rightarrow h k=h_{1} k_{1}
$$

Since $G$ is a group $\exists h^{-1}, k^{-1}, h_{l}^{-1}, k_{l}^{-1} \in G$ such that WLOG $h h^{-1}=e$.

Consider then

$$
\begin{aligned}
& & h^{-1}(h k) k_{l}^{-1} & =h^{-1}\left(h_{1} k_{1}\right) k_{l}^{-1} \\
\Leftrightarrow & & \left(h^{-1} h\right) k k_{l}^{-1} & =h^{-1} h_{1}\left(k_{1} k_{1}^{-1}\right) \\
\Leftrightarrow & & (e) k k_{l}^{-1} & =h^{-1} h_{1}(e) \\
& \Leftrightarrow & k k_{l}^{-1} & =h^{-1} h_{1}
\end{aligned}
$$

Notice that $k k_{l}^{-1} \in K$ and $h^{-1} h_{l} \in H$ and since $H \cap K=\{e\}$, we know that

$$
\begin{aligned}
& k k_{l}^{-1}=e \\
& \left(k k_{l}^{-1}\right) k_{1}=e k_{1} \\
& \text { AND } \\
& h^{-1} h_{1}=e \\
& k\left(k_{l}^{-1} k_{l}\right)=k_{1} \\
& k e=k_{1} \\
& k=k_{1} \\
& h\left(h^{-1} h_{1}\right)=h e \\
& \left(h h^{-1}\right) h_{1}=h \\
& \text { e } h_{1}=h \\
& h_{1}=h
\end{aligned}
$$

Therefore, is $\forall g \in G, g=h k \ni ! h \in H$ and $! k \in K$

Claim: $\phi: G \rightarrow H \times K$ be given by $\phi(g)=(h, k)$ is an isomorphism.

Notice that $\phi$ is well defined since $\forall g \in G, g=h k \ni ! h \in H$ and $! k \in K$, this means that $\phi(g)=\phi(h k)=(h, k)$.

Claim: $\phi$ is one to one

Let $\quad\left(h_{1}, k_{1}\right),\left(h_{2}, k_{2}\right) \in H \times K$
Suppose
$\left(h_{1}, k_{1}\right)=\left(h_{2}, k_{2}\right)$
$\Leftrightarrow \quad \phi\left(h_{1} k_{1}\right)=\phi\left(h_{2} k_{2}\right)$
by the definition of $\phi$
$\Leftrightarrow \quad h_{1} k_{1}=h_{2} k_{2}$
by well definedness of $\phi$ 
Therefore $\phi$ is one to one.

Claim: $\phi$ is onto

Let $\quad(h, k) \in H \times K$

then notice that we are given $\exists g \in G$ such that $g=h k$ and so we can consider $\phi(g)=\phi(h k)=(h, k) \quad$ since $\phi$ is everywhere defined.

Therefore $\phi$ is onto.

Lemma 1: $\quad h k=k h \quad \forall h, k \in G$

$\begin{array}{llll}\text { pf: Let } & h k \in G & \\ \text { notice } & h k=k h^{*} & \text { for some } h^{*} \in H & \text { by } H \triangleleft G \\ \text { also } & h k=k^{*} h & \text { for some } k^{*} \in K & \text { by } K \triangleleft G \\ \text { then } & k h^{*}=k^{*} h & \text { by transitivity } & \end{array}$

Since $G$ is a group $\exists h^{-1}, k^{-1} \in G$ such that WLOG $h h^{-1}=e$.

$\begin{array}{rlrl}\text { Consider then } & & k^{-1}\left(k h^{*}\right) h^{-1}=k^{-1}\left(k^{*} h\right) h^{-1} \\ & \Leftrightarrow & \left(k^{-1} k\right) h^{*} h^{-1}=k^{-1} k^{*}\left(h h^{-1}\right) \\ \Leftrightarrow & (e) h^{*} h^{-1}=k^{-1} k^{*}(e) \\ & \Leftrightarrow & h^{*} h^{-1}=k^{-1} k^{*}\end{array}$

Notice that $h^{*} h^{-1} \in H$ and $k^{-1} k^{*} \in K$ and since $H \cap K=\{e\}$, we know that

$$
\begin{aligned}
& h^{*} h^{-1}=e \\
& h^{*} h^{-1} h=e h \\
& h * e=h \\
& h^{*}=h \\
& k^{-1} k^{*}=e \\
& k k^{-1} k^{*}=k e \\
& e k^{*}=k \\
& k^{*}=k
\end{aligned}
$$

Therefore $h k=k h$.

Claim: $\phi$ is a homomorphism

Let $\quad g, g^{*} \in G \quad$ such that $g=h k$ and $g^{*}=h^{*} k^{*}$ for some $h, k, h^{*}, k^{*} \in G$

$$
\text { Consider } \quad \begin{aligned}
\phi\left(g g^{*}\right)=\phi\left(h k h^{*} k^{*}\right) & =\phi\left(h h^{*} k k^{*}\right) & & \text { by } h k=k h \\
& =\phi\left(\left(h h^{*}\right)\left(k k^{*}\right)\right) & & \text { by associativity of } G \\
& =\left(h h^{*}, k k^{*}\right) & & \text { by definition of } \phi
\end{aligned}
$$

Also consider $\phi(g) \phi\left(g^{*}\right)=\phi(h k) \phi\left(h^{*} k^{*}\right)$

$$
=(h, k)\left(h^{*}, k^{*}\right) \quad \text { by definition of } \phi
$$




$$
=\left(h h^{*}, k k^{*}\right) \quad \text { by combination in } G \times H
$$

Notice since $\left(h h^{*}, k k^{*}\right)=\left(h h^{*}, k k^{*}\right)$, then $\phi\left(g g^{*}\right)=\phi(g) \phi\left(g^{*}\right)$.

Therefore $\phi$ is a homomorphism.

Therefore $\phi: G \rightarrow H \times K$ is an isomorphism. 